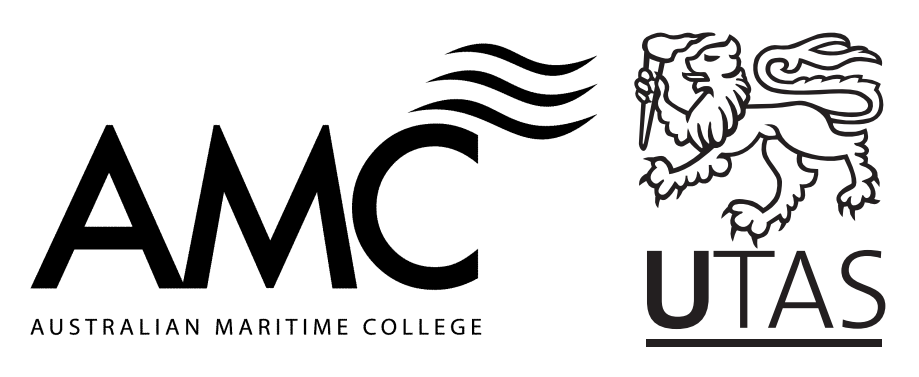

\title{
The Bubble Dynamics and Pressure Field Generated by a Seismic Airgun
}

\author{
Katrina L. de Graaf \\ B.Eng(Naval Architecture)(Hons) \\ National Centre for Maritime Engineering and Hydrodynamics \\ Australian Maritime College
}

Submitted in fulfilment of the requirements

for the degree of Doctor of Philosophy,

University of Tasmania

December, 2013 


\section{Declarations}

\section{Authority of Access}

The publishers of the papers comprising Chapters $3-5$ hold the copyright for that content, and access to the material should be sought from the respective journals. The remaining non published content of this thesis may be made available for loan and limited copying and communication in accordance with the Copyright Act 1968.

\section{Statement of Originality}

This thesis contains no material which has been accepted for a degree or diploma by the University or any other institution, except by way of background information and as duly acknowledged in the text, and to the best of my knowledge and belief no material previously published or written by another person except where due acknowledgement is made in the text of the thesis, nor does the thesis contain any material that infringes copyright.

Signed:

Date:

Katrina de Graaf 


\section{Statement of Co-authorship}

Chapters $3-5$ of this thesis have been written as scientific papers. All numerical simulation, experimental design, data analysis and manuscript preparation were the primary responsibility of the candidate; however, work was performed in collaboration with supervisors. Details of these contributions are outlined below:

\section{Chapter 3}

Modelling of Seismic Airgun Bubble Dynamics and Pressure Field using the Gilmore Equation with Additional Damping Factors

Irene Penesis and Paul Brandner contributed discussions on the numerical modelling and manuscript preparation.

Contribution Percentage: Candidate 70\%, I. Penesis 20\%, P.A. Brandner 10\%

\section{Chapter 4}

The Pressure Field Generated by a Seismic Airgun

Paul Brandner and Irene Penesis contributed to experiment design, data interpretation and manuscript preparation.

Contribution Percentage: Candidate 70\%, P.A. Brandner 20\%, I. Penesis 10\%

\section{Chapter 5}

Bubble Dynamics of a Seismic Airgun

Paul Brandner and Irene Penesis contributed to experiment design, data interpretation and manuscript preparation.

Contribution Percentage: Candidate 70\%, P.A. Brandner 20\%, I. Penesis 10\% 
We the undersigned agree with the above stated "proportion of work undertaken" for each of the above published (or submitted) peer-reviewed manuscripts contributing to this thesis

Signed:

Date:

Dr Irene Penesis

Supervisor

National Centre for Maritime Engineering and Hydrodynamics

Australian Maritime College, University of Tasmania

Signed:

Date:

Assoc. Prof. Paul Brandner

Supervisor

National Centre for Maritime Engineering and Hydrodynamics

Australian Maritime College, University of Tasmania 


\section{Acknowledgements}

Thank you to my supervisors, Irene Penesis and Paul Brandner for helping me to organise a thesis topic and providing support and feedback throughout my candidature. Thank you to Bryce Pearce for his suggestions and assistance and Rob Wrigley for his ideas and seemingly unlimited technical support! Thank you to DSTO, in particular to Stuart Cannon and Warren Reid, for providing my project topic and the associated funding. And finally, thank you to God, who has given me strength and perseverance, and to my family and friends for putting up with my stress levels and providing support and encouragement over the last three years. 


\section{Abstract}

The bubble dynamics of a seismic airgun are investigated in relation to their use for shock testing naval vessels. A model-scale airgun is used to investigate the dynamics of a bubble generated at different standoffs from a steel plate and a free surface in an open top tank. The airgun is fired at 50 and 100 bar initial pressures. Field pressure, wall pressure and acceleration are measured, and the bubble is imaged using low- and highspeed photography. The behaviour of the bubble is described and the bubble growth is determined from shadowgraph photography and compared with the simultaneous pressure field signal. Four distinct bubbles are evident at the first bubble maximum as a result of the initial four jets of air. The Rayleigh-Taylor Instability is identified as playing a role in the bubble break up. The bubble and reverberant frequencies are identified using Wavelet and Fast Fourier transforms. The interaction of the bubble with the free surface is similar compared with other bubbles; however, the bubble is unaffected by the nearby wall for the standoffs tested. Only the initial shock generated by the airgun significantly impacts the wall, which moves in phase with the bubble pulsations. As a result of the movement, little pressure is felt at the wall from the pressure pulses generated by the bubble collapses. Despite the four port arrangement, the pressure signature of this airgun is not directional.

An analytical model of the bubble dynamics is developed based on the Gilmore equation. Additional terms for modelling the presence of the airgun body, mass throttling, effective viscosity and heat diffusion are included and Gilmore's model for the radiated pressure wave is used to predict the pressure field generated by the bubble. The results of this model compare favourably with the model-scale airgun data, with the exception of the initial shock and first maximum bubble velocity, which are over-predicted. Full-scale airgun pressure field measurements are predicted well by the model.

Smoothed Particle Hydrodynamics is investigated as a numerical method for modelling a pulsing seismic airgun bubble. Limitations are found in the available computational time and power, and in the ability to accurately model the gas-water interface. Some improvements are identified to enable modelling the bubble pulsation due to the pressure differential across the bubble surface. 


\section{Contents}

$\begin{array}{ll}\text { Declarations } & \text { ii }\end{array}$

Authority of Access . . . . . . . . . . . . . . ii

Statement of Originality . . . . . . . . . . . . . . ii

Statement of Co-authorship . . . . . . . . . . . . . . iii

Acknowledgements $\quad$ v

$\begin{array}{lll}\text { Abstract } & \text { vi }\end{array}$

Table of Contents vii

List of Figures $\quad \mathrm{x}$

List of Tables $\quad$ xiv

Nomenclature $\quad$ Xv

Abbreviations $\quad$ xvii

1 General Introduction 1

2 Smoothed Particle Hydrodynamics for Modelling Seismic Airguns and Underwater Explosions $\quad 5$

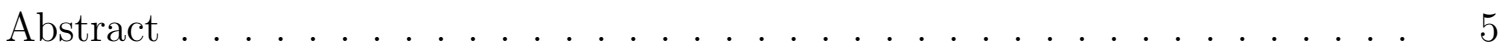

2.1 Introduction . . . . . . . . . . . . . . 5

2.2 SPH Model . . . . . . . . . . . . . . . . . . . . . . 8

2.2.1 Basic Model . . . . . . . . . . . . . . . 8

2.2 .2 Boundaries ......................... 11

2.2.3 Smoothing Length Update and Grad-h Terms . . . . . . . . . . . 12 
2.2.4 Computational Time . . . . . . . . . . . . . . . . 13

2.3 Code Development Results and Discussion . . . . . . . . . . . . . . 14

2.3.1 Configuration ....................... 14

2.3.2 Basic Results . . . . . . . . . . . . . . . . . 15

2.3.3 Limitations in Domain Size . . . . . . . . . . . . . . . 17

2.3.4 Gas-Water Interface . . . . . . . . . . . . . . . . . . . . 18

2.4 Conclusion . . . . . . . . . . . . . . . . . . . . . . . . . . 20

3 Modelling of Seismic Airgun Bubble Dynamics and Pressure Field using the Gilmore Equation with Additional Damping Factors 21

Abstract . . . . . . . . . . . . . . . . . . . . . 21

3.1 Introduction . . . . . . . . . . . . . . . . . . . . . . 22

3.2 Comparison of Rayleigh-Plesset and Gilmore Equations . . . . . . . . . . 24

3.3 Additional Aspects to the Gilmore Equation . . . . . . . . . . . . . 27

3.4 Pressure Field . . . . . . . . . . . . . . . . . . . . . . . . . . . . . . . 32

3.5 Comparison of Pressure-Time History with Experimental Data . . . . . . 33

3.6 Conclusion . . . . . . . . . . . . . . . . . . . . . . . 34

Acknowledgements . . . . . . . . . . . . . . . . . . 34

4 The Pressure Field Generated by a Seismic Airgun 37

Abstract . . . . . . . . . . . . . . . . . . 37

4.1 Introduction . . . . . . . . . . . . . . . . . . . . . . 38

4.2 Experimental Setup . . . . . . . . . . . . . . . . . 39

4.2.1 Laboratory Scale Airgun . . . . . . . . . . . . . . . . 39

4.2.2 Testing Tank and Equipment . . . . . . . . . . . . . . 40

4.3 Results and Discussion . . . . . . . . . . . . . . . . 43

4.3.1 Pressure Signal . . . . . . . . . . . . . . . . 43

4.3 .2 Repeatability ....................... 44

4.3.3 Bubble Pulse and Reverberation . . . . . . . . . . . . . . . . 44

4.3.4 Wavelet Transform and FFT Analysis . . . . . . . . . . . . . . . . 48

4.3.5 Hydrodynamic and Acoustic Pressure . . . . . . . . . . . . . . 54

4.3.6 Wall Movement . . . . . . . . . . . . . . . . . . . . . 55

4.3 .7 Pressure with Distance . . . . . . . . . . . . . . . . 59

4.3.8 Interaction with Free Surface . . . . . . . . . . . . . 59 
4.3 .9 Directivity . . . . . . . . . . . . . . . 65

4.3 .10 Scaling . . . . . . . . . . . . . . . . 65

4.4 Conclusion . . . . . . . . . . . . . . . . . . . . 66

Acknowledgements . . . . . . . . . . . . . . . . 66

5 Bubble Dynamics of a Seismic Airgun $\quad 67$

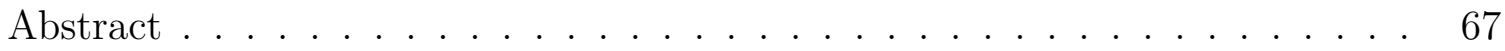

5.1 Introduction . . . . . . . . . . . . . . . . . . 67

5.2 Experimental Setup . . . . . . . . . . . . . . . . . . . 69

5.2.1 Laboratory Scale Airgun . . . . . . . . . . . . . . . . . . . . 69

5.2 .2 Testing Tank and Equipment . . . . . . . . . . . . . . . 70

5.3 Results and Discussion . . . . . . . . . . . . . . . . . . . . . . 73

5.3.1 Bubble Dynamics . . . . . . . . . . . . . 73

5.3.2 Bubble Dynamics Near Boundaries . . . . . . . . . . . 87

5.3.3 Bubble Radial Growth, Velocity and Acceleration with Field Pressure 90

5.4 Comparison with Modified Gilmore Equation . . . . . . . . . . . . . . . . 94

5.4.1 The Modified Gilmore Equation . . . . . . . . . . . . . . . . 94

5.4.2 Comparison of Analytical and Experimental Results . . . . . . . . 96

5.5 Conclusion . . . . . . . . . . . . . . . . . . . . . . . . . . . 99

Acknowledgements . . . . . . . . . . . . . . . . 100

6 General Conclusion $\quad 101$

$\begin{array}{ll}\text { References } & 104\end{array}$

A Australasian Fluid Mechanics Conference Paper: Comparison of the Rayleigh-Plesset and Gilmore Equations and Additional Aspects for the Modelling of Seismic Airgun Bubble Dynamics 


\section{List of Figures}

2.1 Configuration of SPH simulation. . . . . . . . . . . . . . . . . . 14

2.2 Pressure contour plots from an SPH simulation of a highly pressurised gas bubble. . . . . . . . . . . . . . . . . . 16

2.3 Detailed view of Figure 2.2h showing particle density at the gas-water interface. . . . . . . . . . . . . . . . . . . 18

2.4 The interaction of particles with significantly differing particle densities. . 19

3.1 Illustration of airgun firing in three stages. . . . . . . . . . . . . . . . . . 22

3.2 First maximum radius as a function of initial pressure predicted by Rayleigh-Plesset and Gilmore equations with initial radius of $0.1 \mathrm{~m}$. Other variables are taken as: $p_{\infty}=1 \times 10^{5} \mathrm{~Pa}, \rho=1000 \mathrm{~kg} / \mathrm{m}^{3}, p_{v}=1910 \mathrm{~Pa}$, $\mu=1 \times 10^{-3} \mathrm{~kg} / \mathrm{ms}$ and $S=0.074 \mathrm{~N} / \mathrm{m} \ldots \ldots \ldots \ldots$

3.3 Comparison of predicted temporal variation of non-dimensional bubble radius from Rayleigh-Plesset and Gilmore equations for an initial pressure and radius of 100 bar and $0.1 \mathrm{~m}$, respectively. . . . . . . . . . . . . 26

3.4 The effect of various aspects on the solution of the Gilmore equation. . . 27

3.5 The effect of including conductive heat transfer and heat transfer through latent heat on the temperature predicted by the Gilmore equation. Ambient water temperature is $20^{\circ}$ Celsius. . . . . . . . . . . . . . . 30

3.6 The combined effect of including all additional factors on the solution to the Gilmore equation. . . . . . . . . . . . . . . . . . . . . . . . . 31

3.7 The type 520 SERCEL airgun owned by DSTO . . . . . . . . . . . 33

3.8 Comparison of waveform produced by model with results full-scale experimental results. 
4.1 Model-scale airgun. . . . . . . . . . . . . . . . . . . . . . . . . . 39

4.2 Illustration of the model airgun firing in three stages. . . . . . . . . . . . 40

4.3 Experimental Setup. . . . . . . . . . . . . . . . . . . . . . . . 41

4.4 Schematic showing the data acquisition equipment setup. . . . . . . . . . 42

4.5 The bubble generated by the model-scale seismic airgun. . . . . . . . . . 43

4.6 Typical field response at a standoff distance of $191 \mathrm{~mm}$ for initial airgun pressures of 50 and 100 bar. . . . . . . . . . . . . . . . . . . . 45

4.7 Repeatability of the airgun firings shown through the comparison of five raw field pressure responses. . . . . . . . . . . . . . . . . . . . . . . 46

4.8 Repeatability of the airgun firings shown through the comparison of five filtered field pressure responses. . . . . . . . . . . . . . . . . . . . 47

4.9 Field pressure response for airgun fired at 50 bar initial pressure, with bubble pulse and reverberation extracted.

4.10 Field pressure response for airgun fired at 100 bar initial pressure, with bubble pulse and reverberation extracted.

4.11 Wavelet transforms for field pressure, wall pressure and acceleration for 50 bar initial airgun pressure. . . . . . . . . . . . . . . . . .

4.12 Wavelet transforms for field pressure, wall pressure and acceleration for 100 bar initial airgun pressure. . . . . . . . . . . . . . . . . . . 52

4.13 Comparison of PSDs from the wavelet transform and FFT. . . . . . . . . 53

4.14 PSD from the wavelet transform for the field pressure responses at varying distances from the airgun fired at 100 bar initial pressure. . . . . . . . . . 56

4.15 Field pressure, wall pressure, wall acceleration and wall movement for 50 and 100 bar initial airgun pressures. The airgun is located at the centre of the tank.

4.16 Field pressure, wall pressure, wall acceleration and wall movement for 50 and 100 bar initial airgun pressures. The airgun is located $200 \mathrm{~mm}$ from the steel wall.

4.17 Pressure with distance of the initial shock for the airgun fired at 50 and 100 bar initial pressures. . . . . . . . . . . . . . . .

4.18 Pressure with distance of the pulse generated by the first bubble collapse for the airgun fired at 50 and 100 bar initial pressures. . . . . . . . . . 
4.19 Initial shock pressure at different depths relative to the fixed-depth airgun fired at an initial pressure of 100 bar. . . . . . . . . . . . . . . . 62

4.20 Averaged field pressure response at $184 \mathrm{~mm}$ for varying standoff distances from the free surface for 100 bar initial pressure. . . . . . . . . .

4.21 Pressure pulse at a horizontal standoff distance of $184 \mathrm{~mm}$ generated by the first bubble collapse, with respect to airgun standoff distance from the free surface for 100 bar initial pressure. . . . . . . . . . . . .

4.22 Bubble period with respect to airgun standoff distance from the free surface for 100 bar initial pressure. . . . . . . . . . . . . . . . . 64

4.23 Directivity of the airgun when fired at 100 bar initial pressure. . . . . . .

5.1 Single frame of video footage provided by SERCEL of GGun150 fired at

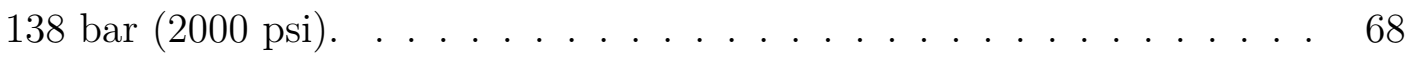

5.2 Model-scale airgun. . . . . . . . . . . . . . . . . . 70

5.3 Illustration of the model-scale airgun firing in three stages. . . . . . . . . 71

5.4 Experimental Setup. . . . . . . . . . . . . . . . . . 72

5.5 Schematic showing the setup of the data acquisition equipment. . . . . . 73

5.6 Still images of bubble produced by the scale airgun fired at an initial pressure of 100 bar. . . . . . . . . . . . . . . 76

5.7 Relative point in the bubble's lifetime of images in Figure 5.6. . . . . . . 82

5.8 Images from $20 \mathrm{kHz}$ high-speed photography (50 $\mu$ s increments) showing initial release of air after firing at 100 bar initial pressure. . . . . . . . . .

5.9 Images from high-speed photography at intervals of $0.33 \mathrm{~ms}$ showing jet of air penetrating initial growing bubble interface for 100 bar initial airgun pressure. . . . . . . . . . . . . . . . .

5.10 Images from high-speed photography at intervals of $1.67 \mathrm{~ms}$ showing formation of re-entrant jet during first bubble collapse for 100 bar initial airgun pressure. . . . . . . . . . . . . . .

5.11 Images from high-speed photography at intervals of $1.67 \mathrm{~ms}$ showing formation of re-entrant jet during first bubble collapse and subsequent second bubble growth at a standoff distance of 0.75 bubble radii from the free surface for 100 bar initial airgun pressure. 
5.12 Bubble radius, velocity and acceleration from photography at frame rates of $3 \mathrm{kHz}$ and $20 \mathrm{kHz}$ for 50 bar initial airgun pressure with corresponding free-field pressure response. . . . . . . . . . . . . . . . . . . . . . 92

5.13 Bubble radius, velocity and acceleration from photography at frame rates of $3 \mathrm{kHz}$ and $20 \mathrm{kHz}$ for 100 bar initial airgun pressure with corresponding free-field pressure response. . . . . . . . . . . . . . . . . . . . 93

5.14 Comparison of radius and velocity with results of the modified Gilmore equation for an initial airgun pressure of 50 bar . . . . . . . . . . . 97

5.15 Comparison of radius and velocity with results of the modified Gilmore equation for an initial airgun pressure of 100 bar . . . . . . . . . . . 98 


\section{List of Tables}

2.1 SPH parameters for shock wave propagation simulation. . . . . . . . . 15

3.1 Parameters for the bubble model for the type 520 SERCEL airgun. . . . 32

3.2 Configuration parameters for airgun firings. . . . . . . . . . . . . 34

4.1 Bubble and reverberant field frequencies obtained from the wavelet transform and FFT. . . . . . . . . . . . . . . . . . . . 55

5.1 Table of parameters used in the modified Gilmore equation to predict the bubble behaviour of the model-scale airgun. . . . . . . . . . . . . . 96 


\section{Nomenclature}

\begin{tabular}{|c|c|c|}
\hline$A$ & area & $\mathrm{m}^{2}$ \\
\hline$A_{0}$ & orifice area & $\mathrm{m}^{2}$ \\
\hline$c$ & local speed of sound & $\mathrm{ms}^{-1}$ \\
\hline$c_{\infty}$ & speed of sound & $\mathrm{ms}^{-1}$ \\
\hline$D$ & diameter & $\mathrm{m}$ \\
\hline$e$ & specific internal energy & $\mathrm{Jkg}^{-1}$ \\
\hline$f$ & frequency & $\mathrm{Hz}$ \\
\hline$f_{0}$ & natural frequency & $\mathrm{Hz}$ \\
\hline$f(\mathbf{x})$ & SPH field function & \\
\hline$h$ & smoothing length & $\mathrm{m}$ \\
\hline$H$ & enthalpy difference & $\mathrm{J}$ \\
\hline$k$ & polytropic index & \\
\hline$L$ & latent heat & $\mathrm{Jkg}^{-1}$ \\
\hline$m$ & mass & $\mathrm{kg}$ \\
\hline$m_{G}$ & mass of air in airgun chamber & $\mathrm{kg}$ \\
\hline$\dot{m}$ & mass flow rate & $\mathrm{kgs}^{-1}$ \\
\hline$\dot{m}_{v}$ & vapour mass flow rate & $\operatorname{kgs}^{-1}$ \\
\hline$p$ & pressure & $\mathrm{Pa}$ \\
\hline$p_{0}$ & initial pressure & $\mathrm{Pa}$ \\
\hline$p_{a}$ & atmospheric pressure & $\mathrm{Pa}$ \\
\hline$p_{g}$ & initial airgun pressure & $\mathrm{Pa}$ \\
\hline$p_{G}$ & pressure of airgun chamber & $\mathrm{Pa}$ \\
\hline$p_{l}$ & local water pressure & $\mathrm{Pa}$ \\
\hline$p_{v}$ & vapour pressure & $\mathrm{Pa}$ \\
\hline$p_{\infty}$ & hydrostatic pressure & $\mathrm{Pa}$ \\
\hline $\operatorname{Pr}$ & Prandtl Number & \\
\hline$\dot{Q}$ & rate of heat conduction & $\mathrm{Js}^{-1}$ \\
\hline$\dot{Q}_{C}$ & rate of classical heat conduction & $\mathrm{Js}^{-1}$ \\
\hline$\dot{Q}_{L}$ & rate of latent heat conduction & $\mathrm{Js}^{-1}$ \\
\hline
\end{tabular}




\begin{tabular}{|c|c|c|}
\hline$r$ & radial distance & $\mathrm{m}$ \\
\hline$r_{0}$ & equivalent equilibrium bubble radius & $\mathrm{m}$ \\
\hline$R$ & bubble radius & $\mathrm{m}$ \\
\hline$R_{g}$ & equivalent bubble radius of airgun chamber & $\mathrm{m}$ \\
\hline$R_{G}$ & ideal gas constant & $\mathrm{Jkg}^{-1} \mathrm{~K}^{-1}$ \\
\hline$R_{M}$ & maximum bubble radius & $\mathrm{m}$ \\
\hline$R e$ & \multicolumn{2}{|l|}{ Reynolds number } \\
\hline$\dot{R}$ & bubble velocity & $\mathrm{ms}^{-1}$ \\
\hline$\dot{R}_{R}$ & Rayleigh collapse velocity & $\mathrm{ms}^{-1}$ \\
\hline$\ddot{D}$ & bubble acceleration & $\mathrm{ms}^{-2}$ \\
\hline$\ddot{R}_{R}$ & Rayleigh collapse acceleration & $\mathrm{ms}^{-2}$ \\
\hline$S$ & surface tension & $\mathrm{mNm}^{-1}$ \\
\hline$t$ & time & $\mathrm{s}$ \\
\hline$t^{*}$ & \multicolumn{2}{|l|}{ non-dimensional time } \\
\hline$T$ & temperature & K \\
\hline$T_{w}$ & water temperature & $\mathrm{K}$ \\
\hline$u_{l}$ & local liquid velocity & $\mathrm{ms}^{-1}$ \\
\hline $\mathbf{v}$ & SPH particle velocity vector & $\mathrm{ms}^{-1}$ \\
\hline$V$ & volume & $\mathrm{m}^{3}$ \\
\hline$V_{A G}$ & volume of airgun body & $\mathrm{m}^{3}$ \\
\hline$V_{G}$ & volume of airgun chamber & $\mathrm{m}^{3}$ \\
\hline$W$ & smoothing kernel function & $\mathrm{m}^{-1}, \mathrm{~m}^{-2}, \mathrm{~m}^{-3}$ \\
\hline$x$ & horizontal distance & $\mathrm{m}$ \\
\hline $\mathrm{x}$ & SPH particle position vector & $\mathrm{m}$ \\
\hline$z$ & vertical distance & $\mathrm{m}$ \\
\hline$z_{f s}$ & free surface standoff distance & $\mathrm{m}$ \\
\hline$\alpha_{\pi}$ & constant for SPH artificial viscosity & \\
\hline$\beta_{\pi}$ & constant for SPH artificial viscosity & \\
\hline$\varepsilon$ & constant for XSPH & \\
\hline$\mu$ & viscosity & Pas \\
\hline$\mu_{d y n}$ & dynamic viscosity & Pas \\
\hline$\mu_{e f f}$ & effective viscosity & Pas \\
\hline$\Pi_{i j}$ & SPH artificial viscosity & $\mathrm{kgm}^{-3}$ \\
\hline$\rho$ & density & $\mathrm{kgm}^{-3}$ \\
\hline$\tau$ & bubble period & $\mathrm{s}$ \\
\hline & Rayleigh collapse time & $\mathrm{s}$ \\
\hline & non-dimensional period & \\
\hline
\end{tabular}




\section{Abbreviations}

$\begin{array}{ll}\text { AMC } & \text { Australian Maritime College } \\ \text { B\&K } & \text { Brüel \& Kjær } \\ \text { CFD } & \text { Computational Fluid Dynamics } \\ \text { DSTO } & \text { Defence Science and Technology Organisation } \\ \text { FFT } & \text { Fast Fourier Transform } \\ \text { NI } & \text { National Instruments } \\ \text { OpenMP } & \text { Open Multi-Processing } \\ \text { MPI } & \text { Message Passing Interface } \\ \text { RAN } & \text { Royal Australian Navy } \\ \text { SPH } & \text { Smoothed Particle Hydrodynamics } \\ \text { U.K. } & \text { United Kingdom } \\ \text { U.S. } & \text { United States }\end{array}$




\section{General Introduction}

Seismic airguns are an acoustic source that create a pressure pulse by rapidly releasing a highly compressed volume of air underwater. They are used in marine seismic surveys for analysing the subsurface structure and more recently they are being developed as an alternative to underwater explosions for the shock testing of defence vessels $[69,80]$. Airguns are a simpler alternative for shock trials when compared with high explosives as they are less costly, safer, more environmentally friendly and easier and less time-consuming to set up. The U.S. and U.K. navies are currently investigating the use of seismic airguns in shock trials and methods have been developed by the U.S. Navy and contractors Weidlinger Associates (Patent US6662624 [86]). The Royal Australian Navy (RAN) is also interested in conducting shock trials with seismic airguns and research in this area is being undertaken by the Defence Science and Technology Organisation (DSTO). DSTO have purchased several commercial SERCEL airguns and have completed two full-scale airgun trials in which free-field pressures and the response of a small-scale hull have been measured $[12,71]$. The work presented in this thesis has been funded by the Maritime Platforms Division of DSTO and the Australian Maritime College (AMC) to further basic research on seismic airguns in the field of shock testing. This has been achieved through the development of a model-scale airgun, laboratory testing, development of an analytical bubble dynamics model and investigation into a numerical model based on Smoothed Particle Hydrodynamics (SPH).

A model-scale airgun has been designed based on the full-scale SERCEL airguns and a series of experiments has been undertaken in a purpose-built tank at the AMC to investigate the field and wall pressures generated by the airgun when fired at different standoff distances from a steel wall and from the free surface. The testing facility also allowed the bubble dynamics to be investigated through low- and high-speed photography. 
While much research exists in the field of bubble dynamics, the particular dynamics that affect a seismic airgun bubble are not well documented. Additional phenomena are introduced for an airgun bubble that are not present in bubbles generated by other means (such as cavitation, underwater explosion, and spark- and laser- generation). These phenomena form as a result of the formation of the bubble, during which high pressure air is forced through one or more 'ports', and also as a result of the presence of the airgun body at the centre of the bubble. Some photography of airgun bubbles has been published $[6,45]$; however, the majority of understanding has been obtained through experimental measurement of the pressure field (primarily using full-scale airguns) and subsequent development of numerical simulation methods, for example: [36, 43, 44, 75, 84, 89].

Basic bubble models for period and pressure pulse prediction have been applied to seismic airguns by the geophysical community with some success. The use of empirical derivations and more complicated numerical models [51, 53, 68], including Computational Fluid Dynamics (CFD) [13, 34], improve the pulse prediction and provide additional understanding of the bubble physics; however, there are sources of damping which are still not fully explained. For shock testing of ships, the development of numerical predictions provides significant benefits in identifying optimum parameters for the most efficient shock trial configurations, and also in reducing the number of trials required. While these models undoubtedly require the ability to model the arrays of airguns that are used to generate the required shock wave strength, understanding the interaction of multiple airgun bubbles relies on an understanding of a single bubble. As part of this work, an analytical model based on the Gilmore equation [27] (formulated for modelling underwater explosion bubbles) has been developed and used to predict fullscale and model-scale seismic airgun bubble dynamics. Additional factors specific to airgun bubbles and/or bubbles formed by highly pressurised gas, are incorporated into the model to investigate their impact on the bubble dynamics and damping mechanisms. Gilmore's model for the radiated pressure wave has also been used to predict the pressure field generated by the bubble.

SPH is a particle based numerical method that has been explored as a numerical option for modelling seismic airgun bubbles. Unlike traditional meshed methods, the particles in SPH are not linked together to calculate material properties at each node. This results in a method that is inherently ideal for modelling the large deformities that develop in the longer-term simulation of underwater explosion [59, 79] — and seismic airgun bubbles. 
The research objectives of this work are:

- To improve the understanding of the behaviour of a bubble formed by a seismic airgun.

- To investigate the viability of using seismic airguns to shock test naval craft.

- To perform experiments that model characteristics of a seismic airgun; particularly the coalescing of air from multiple ports.

- To perform experiments that show the effect of a free surface and rigid boundary at varying distances from an airgun bubble.

- To develop a numerical model using SPH that simulates the bubble formed by a seismic airgun, including the coalescing of air from multiple airgun ports and the interaction of the bubble with a free surface and rigid boundary.

The research questions answered by this research are:

- How do the multiple ports in an airgun interact to form one bubble?

- What parameters affect the dynamics of the bubble formed through multiple ports?

- What parameters affect the shock and pressure field produced by the bubble?

- What effect does the presence of a body (the airgun) have on the behaviour of the bubble?

- How does the bubble interact with a flat, surface piercing plate at varying standoff distances and depths?

- Can the behaviour and characteristics of the airgun bubble process be scaled?

- Can SPH be used to model the pulsating bubble produced by a seismic airgun?

- How do the numerical and experimental results compare?

This thesis is comprised of a number of chapters, most of which are papers that have been submitted to be published elsewhere. The relevant publishing details are given at the beginning of each chapter. An outline of the chapters, and their contribution to the research objectives, is given below: 
- Chapter 2 presents the work done to develop an SPH code to model the bubble formed by a seismic airgun. The simulation of the bubble was not successful due to several limitations in the current ability of SPH. These are outlined in the chapter.

- Chapter 3 presents an analytical model of the bubble growth and generated pressure field of a seismic airgun bubble. The model is used to investigate different parameters that affect the bubble and radiated pressure waveform. These parameters include the throttling of the firing air through the ports, the presence of the airgun body at the centre of the bubble, the effective viscosity generated by turbulence and instabilities on the bubble's surface, and heat conduction and vapour mass transfer across the bubble wall. The model is compared with full-scale airgun experimental data (externally provided).

- Chapter 4 presents a detailed analysis of the field pressure generated in experiments performed with a model-scale airgun. The interaction of the airgun bubble, fired at multiple standoff distances, with the tank wall (a surface piercing flat plate) and the free surface is also presented. This work provides data that can be used in conjunction with full-scale results for further investigation into the scaling relationships of airgun bubbles.

- Chapter 5 presents low- and high-speed photography of the bubble dynamics generated by the model-scale airgun. This work provides a detailed analysis of the dynamics of the bubble, including the interaction of the four jets of air, and the bubble's response to the presence of the airgun body at its centre. Quantitative measurements of the bubble growth and field pressure results are compared with the analytical model developed in Chapter 3. 


\title{
Smoothed Particle Hydrodynamics for Modelling Seismic Airguns and Underwater Explosions
}

This chapter describes the work done in developing a Smoothed Particle Hydrodynamics code for modelling the bubble created by a seismic airgun. This work forms a chapter of this thesis, but will not be considered for publication elsewhere.

\begin{abstract}
Smoothed Particle Hydrodynamics (SPH) has been investigated as a numerical method for modelling the dynamics of a pulsing gas bubble surrounded by water. The bubble is modelled in an open top tank with a free surface. A freely available SPH code has been modified with techniques discussed in the literature to include a tree-search algorithm and an improved method for dealing with adjacent fluids of different densities. Limitations were found in the available computational time and power. The improvements required to sufficiently model the gas-water interface to achieve bubble collapse due to the pressure differential are identified.
\end{abstract}

\subsection{Introduction}

The Australian Government Defence Science and Technology Organisation (DSTO) is currently investigating the use of seismic airguns as an alternative to underwater explosions for shock testing naval vessels, due to their comparatively lower cost, fewer safety risks, and more environmentally friendly operation. Airguns are primarily used in geophysical surveying to rapidly release highly pressurised air which forms a bubble 
that pulses in the same manner as the bubble formed as the result of an underwater explosion. Upon firing the airgun or detonating the explosives, an initial shock wave is emitted and the highly pressurised gas bubble expands rapidly. The bubble expands at such a rate that the inertia in the surrounding water causes it to over expand to the point where the bubble pressure is lower than the hydrostatic pressure. This causes the bubble to collapse violently, and it reaches the state where the pressure of the bubble contents is again higher than that of the water. The bubble collapse generates a pressure wave in the water, and the bubble rebounds. The expansion and collapse cycle continues with a pressure pulse radiated at each collapse, until the bubble's energy has dissipated and it breaks up and rises to the surface due to buoyancy.

In order to support the airgun shock testing trials, DSTO is interested in developing a numerical modelling capability to simulate the behaviour and dynamics of airgun bubbles. This would provide a basis for the comparison of different airgun configurations and give an indication of the expected trial outcomes. Smoothed Particle Hydrodynamics ( $\mathrm{SPH})$ has been identified as a possible computational method for this capability.

$\mathrm{SPH}$ is a meshless particle method of Lagrangian nature that approximates the solutions of the equations of fluid dynamics: the continuity equation, the momentum equation and the energy equation. It was originally developed for the astrophysics community [28]; however, it is now used for a wide range of problems, including hydrodynamic simulations such as sloshing, slamming and fluid flow. While mesh-based techniques are capable of modelling some aspects of underwater explosions, they have limitations. Lagrangian grids are fixed to the object and suffer from distortion when modelling bubble pulsations, which can dramatically increase computation time and lead to simulation failure. Eulerian grids are more suited to fluid dynamics; however, diffusion and limited mesh resolution prevent accurate modelling of material interfaces and structural response. SPH allows particles to move independently, overcoming the disadvantages of meshed methods and allowing inhomogeneous effects and deformations to be modelled. These properties suggest that the method would be ideal for modelling the asymmetric phenomena of a pulsing bubble near a boundary and also the breakup of the free surface. Swegle and Attaway [79] investigated the use of SPH coupled with a finite element code, PRONTO, to model the bubble dynamics created by an underwater explosion in conjunction with the deformation of a thin structure. The extensive simulation time was identified as prohibiting as it was not possible to accurately predict both the initial shock wave and the later-time phenomena within the same simulation. Despite this, with further work, SPH was identified as having great potential in this area. 
Liu and Liu [55] and Liu et al. [59] modelled underwater explosions with SPH and captured major features of the magnitude and location of the pressure field and bubble. Several modifications to the basic SPH method were required to achieve this capability, including evolution of the smoothing length, implementation of solid boundaries and treatment of material interfaces. Although they successfully replicated a pulsing bubble, the simulations were modelled in a fully enclosed box and relied on reflections of the shock wave from the solid boundaries to initiate bubble collapse, rather than the pressure difference across the bubble interface. This led to greatly shortened bubble periods when compared with a free-field underwater explosion. The gas pressure at the bubble's maximum radius also remained significantly higher than the hydrostatic pressure.

Prior to their modelling of underwater explosions, Liu et al. [56] presented simulations of a one-dimensional TNT slab detonation which compared well to previous numerical results. SPH was also used to investigate the effectiveness of water mitigation in reducing the magnitude of the peak shock wave from a high explosive detonation [57]. A process for updating the smoothing length combined with a tree search method was incorporated in simulations for modelling the major physics of an explosion [58]. In this work, both one- and two-dimensional simulations of TNT detonation in a vacuum were compared to results from other numerical methods and good agreement was found with published solutions of other numerical methods.

Liu and Liu [55] consequently modelled a two-dimensional cylindrical underwater explosion. A numerical instability was noticed at the interface of the gas and water which may have resulted in errors in the pressure transient; however, this was considered acceptable when compared to the magnitude of the shock wave and was shown not to affect the predicted magnitude or location of the primary shock wave. This instability was found to be influenced by variables such as the time-step, smoothing length, particle resolution, artificial viscosity and the penetration force applied at the interface.

This work seeks to further the development of multi-phase SPH for use in modelling the bubbles formed by underwater explosions and seismic airguns, i.e. a pressurised gas bubble that pulses due to the pressure differential between the gas and water. 


\subsection{SPH Model}

\subsubsection{Basic Model}

A freely available SPH code that is configured for one- and two-dimensional single fluid examples has been modified to model the pulsing bubble. The code was re-configured in previous work [15] to model an underwater explosion bubble. This was done based on examples provided with the code [55] and included a new particle layout, inclusion of the third dimension, incorporation of a second fluid, and inclusion of new equations of state for the gas and water. In the present work, the code has been further developed taking into account methods in the wider literature, for example, [10, 39, 67].

In the SPH method, each of the field functions (for example, density, mass, energy) are represented in an integral form, where the field, $f$, is a function of the position vector, $\mathbf{x}$. The exact integral form has no definite limits and is approximated by a finite function known as the kernel approximation:

$$
f(\mathbf{x})=\int_{\Omega} f\left(\mathbf{x}^{\prime}\right) W\left(\mathbf{x}-\mathbf{x}^{\prime}, h\right) \mathrm{d} x
$$

where the field function, $f\left(\mathbf{x}^{\prime}\right)$, is multiplied by a smoothing kernel function, $W\left(\mathbf{x}-\mathbf{x}^{\prime}, h\right)$. The approximate value of any of the field variables at any point can be found through integration of the kernel approximation.

The smoothing kernel can take many forms; Morris [65] observed instabilities in SPH methods that exactly conserve momentum which could be improved when using kernels of higher order spline functions that more closely approximate the Gaussian. However, other authors have determined that no kernel is significantly better than the cubic spline [63]. In this work the cubic spline has been used [55], given by:

$$
W(r, h)=\alpha_{d} \begin{cases}\frac{2}{3}-R^{2}+\frac{1}{2} R^{3} & 0 \leq R<1 \\ \frac{1}{6}(2-R)^{3} & 1 \leq R<2 \\ 0 & R \geq 2\end{cases}
$$

where $r=|\mathrm{d} \mathbf{x}| ; R=\frac{|\mathrm{d} \mathbf{x}|}{h} ; \alpha_{d}=\frac{1}{h}, \frac{15}{7 \pi h^{2}}$ and $\frac{3}{2 \pi h^{3}}$ for one-, two- and three- dimensional space, respectively.

The kernel approximation is then used to develop the particle approximation, which for a particular point or particle, uses the summation over neighbouring particles to determine the field variables, rather than integration. The summation is conducted over 
a limited domain, the size of which is determined by the smoothing length, $h$. The particle approximation for a function is given by:

$$
f\left(\mathbf{x}_{i}\right)=\sum_{j=1}^{N} \frac{m_{j}}{\rho_{j}} f\left(\mathbf{x}_{j}\right) \cdot W_{i j}
$$

where $f\left(\mathbf{x}_{i}\right)$ is the field property at particle $i$, and $m_{j}$ and $\rho_{j}$ are the mass and density at the surrounding particles, $j$. The smoothing kernel becomes $W_{i j}=W\left(\mathbf{x}_{i}-\mathbf{x}_{j}, h\right)$.

This approximation can then be applied to the Euler equations (for which viscosity is ignored) for the conservation of mass, conservation of momentum and conservation of energy. The respective equations for the approximations are given by:

$$
\begin{gathered}
\rho_{i}=\sum_{j=1}^{N} m_{j} W_{i j} \\
\frac{\mathrm{d} \mathbf{v}_{i}}{\mathrm{~d} t}=\sum_{j=1}^{N} m_{j} \frac{\left(p_{i}+p_{j}\right)}{\rho_{i} \rho_{j}} \nabla_{i} W_{i j} \\
\frac{\mathrm{d} e_{i}}{\mathrm{~d} t}=\frac{1}{2} \sum_{j=1}^{N} m_{j} \frac{\left(p_{i}+p_{j}\right)}{\rho_{i} \rho_{j}} \mathbf{v}_{i j} \cdot \nabla_{i} W_{i j}
\end{gathered}
$$

where $\mathbf{v}_{i}, p_{i}$ and $e_{i}$ are the velocity vector, pressure and specific internal energy, respectively, at particle $i$. The accuracy and performance of the code is therefore partly dependent on the chosen smoothing length; too large and all details and local properties of the domain will be averaged out; too small and the particles will not interact with one another and the code will fail. The smoothing length is therefore linked to the particle spacing. For this work an initial smoothing length of $1.2|\mathrm{~d} \mathbf{x}|$ is used.

An alternative for Equation 2.4 is the 'continuity density' equation of the form:

$$
\frac{\mathrm{d} \rho_{i}}{\mathrm{~d} t}=\sum_{j=1}^{N} m_{j} \mathbf{v}_{i j} \cdot \nabla_{i} W_{i j}
$$

This form of the equation is based on the density gradient and is more suitable for problems modelling fluid discontinuities, such as shock waves [55]. As the continuity density equation does not exactly conserve mass, Equation 2.4 can be applied at regular intervals to reduce the error accumulation. The use of Equation 2.4; however, presents its own disadvantages, as the assumption that $\sum W_{j}=1$ does not hold at a free surface, and additional errors can be introduced. In place of this, Colagrossi and Landrini [10] apply a moving-least-square method to re-initialise the density field: 


$$
\rho_{i}=\sum_{j=1}^{N} m_{j} W_{j}^{M L S}\left(\mathbf{x}_{i}\right)
$$

where

$$
\begin{aligned}
& W_{j}^{M L S}\left(\mathbf{x}_{i}\right)=\left[\beta_{0}\left(\mathbf{x}_{i}\right)+\beta_{1}\left(\mathbf{x}_{i}\right)\left(x_{i}-x_{j}\right)+\beta_{2}\left(\mathbf{x}_{i}\right)\left(y_{i}-y_{j}\right)\right] W_{i j} \\
& \beta\left(\mathbf{x}_{i}\right)=\left(\begin{array}{c}
1 \\
\beta_{0} \\
\beta_{1} \\
\beta_{2}
\end{array}\right)=\mathbf{A}^{-1}\left(\mathbf{x}_{i}\right)\left[\begin{array}{c}
0 \\
0
\end{array}\right] \\
& \mathbf{A}\left(\mathbf{x}_{i}\right)=\sum_{j=1}^{N} W_{j}\left(\mathbf{x}_{i}\right) \tilde{\mathbf{A}}_{i j} \\
& \tilde{\mathbf{A}}_{i j}=\left[\begin{array}{ccc}
1 & \left(x_{i}-x_{j}\right) & \left(y_{i}-y_{j}\right) \\
\left(x_{i}-x_{j}\right) & \left(x_{i}-x_{j}\right)^{2} & \left(x_{i}-x_{j}\right)\left(y_{i}-y_{j}\right) \\
\left(y_{i}-y_{j}\right) & \left(x_{i}-x_{j}\right)\left(y_{i}-y_{j}\right) & \left(y_{i}-y_{j}\right)^{2}
\end{array}\right]
\end{aligned}
$$

where $x_{i}$ and $y_{i}$ are the components of the position vector $\mathbf{x}_{i}$. Equation 2.9 is twodimensional; the corresponding three-dimensional moving least squares kernel is also used when required.

To use the SPH method to model hydrodynamic problems, water must be assumed to be minutely compressible. This is achieved with an appropriate equation of state that allows a time derivative of pressure to be developed, which is non-existent in incompressible flows. Monaghan [64] uses a modified form of Tait's equation of state, given by:

$$
p=B\left(\left(\frac{\rho}{\rho_{0}}\right)^{n}-1\right)
$$

where $\rho_{0}$ is the initial water density, $n$ and $B$ are constants, taken as 7 and $3.13 \times 10^{8}$ $\mathrm{Pa}$, respectively.

The gas in the bubble is assumed to follow the ideal gas law:

$$
p=(k-1) e_{i} \rho_{i}
$$

where $k$ is the polytropic index, taken as the adiabatic index, 1.4, and $e_{i}$ is the specific internal energy at particle $i$.

To accurately model shock waves, an artificial viscosity term, $\Pi_{i j}$, is included. This represents the energy loss from the shock wave as a form of viscous dissipation and prevents numerical instabilities. The form used in this code is that of Monaghan [62], 
given by:

$$
\Pi_{i j}=\left\{\begin{array}{lc}
\frac{-\alpha_{\pi} \bar{c}_{i j} \phi_{i j}+\beta_{\pi} \phi_{i j}^{2}}{\bar{\rho}_{i j}} & \mathbf{v}_{i j} \cdot \mathbf{x}_{i j}<0 \\
0 & \mathbf{v}_{i j} \cdot \mathbf{x}_{i j} \geq 0
\end{array}\right.
$$

where $\phi_{i j}=\frac{\bar{h}_{i j} \mathbf{v}_{i j} \cdot \mathbf{x}_{i j}}{\left|\mathbf{x}_{i j}\right|^{2}+0.01 h_{i j}^{2}}, \alpha_{\pi}$ and $\beta_{\pi}$ are constants, $\bar{c}_{i j}, \bar{\rho}_{i j}$ and $\bar{h}_{i j}$ are the average speed of sound, density and smoothing length, respectively, of particles $i$ and $j, \mathbf{v}_{i j}=\mathbf{v}_{i}-\mathbf{v}_{j}$ and $\mathbf{x}_{i j}=\mathbf{x}_{i}-\mathbf{x}_{j}$.

For modelling incompressible flows, Monaghan [63] also introduced a technique called XSPH which reduces particle penetrations and maintains a more orderly particle distribution. The XSPH technique introduces dispersion by adjusting each particle velocity using the average local velocity, thereby controlling the degree to which the particle moves with the overall velocity field versus its own velocity. The XSPH form is given by:

$$
\frac{\mathrm{d} \mathbf{x}_{i}}{\mathrm{~d} t}=\mathbf{v}_{i}-\varepsilon \sum_{j=1}^{N} \frac{m_{j}}{\rho_{j}} \mathbf{v}_{i j} W_{i j}
$$

where $\varepsilon$ is a constant, to be determined, in the range of $0 \leq \varepsilon \leq 1.0$.

\subsubsection{Boundaries}

The inclusion of solid boundaries is required in most hydrodynamic simulations and several methods have been proposed. Liu and Liu [55] use two types of virtual particles. Type I virtual particles form the actual boundary and exert a repulsive force on internal particles. These particles have fixed properties and do not evolve. Type II virtual particles are used to prevent particle deficiencies near the boundary. These particles mirror the properties of the real particles and are updated at every time-step.

This code has used only fixed position virtual particles for the boundaries. As the focus of the modelling is the bubble dynamics, high accuracy in the boundary regions is not required; therefore, the more computationally intensive Type II mirrored virtual particles have been replaced with a second layer of the stationary virtual particles. Two layers of stationary virtual particles, with the same properties as the real water particles, were sufficient to prevent particles penetrating the boundary. The virtual particles were similar to the Type I of Liu and Liu [55]; however, no repulsive force was used, as this caused unnatural pressure fluctuations to develop within the domain. With a fully enclosed domain, as modelled by Liu and Liu, the repulsive force is balanced and therefore this is not a problem. 


\subsubsection{Smoothing Length Update and Grad-h Terms}

As the bubble expands, the spacing of the gas particles increases and, if limited to a fixed smoothing length, the particles quickly lose interaction with each other. A method for evolving the smoothing length, based on the density variation [4] is implemented to prevent this. The rate of change of the smoothing length is given by:

$$
\frac{\mathrm{d} h}{\mathrm{~d} t}=-\frac{1}{d} \frac{h}{\rho} \frac{\mathrm{d} \rho}{\mathrm{d} t}
$$

where $d=1,2$ and 3 for one-, two- and three- dimensional space, respectively.

When updating the smoothing length, extra terms are developed in the derivative of the kernel $(W)$ in Equations $2.5-2.7$ as $h$ is no longer constant. These terms are frequently ignored; however, Nelson and Papaloizou $[66,67]$ found that the inclusion of these 'grad- $h$ ' terms result in improvements in a number of simulations previously known to suffer from poor energy conservation. Inclusion of the terms was found to only lead to a slight increase in computational time. They concluded that grad- $h$ terms could not be justifiably ignored on the grounds of small local spatial variations in the smoothing length; rather, the time period over which the smoothing length varies must be considered.

Almi et al. [2] also emphasised the importance of including grad-h terms in the SPH equations when using a spatially varying smoothing length. A comparison found that ignoring these terms resulted in the introduction of a non-physical negative entropy, and density peaks associated with strong shock fronts were over predicted in star-formation simulations.

In order to calculate the grad- $h$ terms, $h$ must be defined as a function of distance. This is given by Nelson and Papaloizou [67] as

$$
h_{i}=\frac{1}{2}\left|\mathbf{x}_{i}-\mathbf{x}_{\text {jmax }}\right|
$$

where $j \max$ is particle $i$ 's most distant neighbour, or

$$
h_{i}=\frac{1}{N_{f a r}} \sum_{j=1}^{N_{f a r}} \frac{1}{2}\left|\mathbf{x}_{i}-\mathbf{x}_{j}\right|
$$

where the summation is over particle $i$ 's $N_{\text {far }}$ most distant neighbours.

Implementation of grad- $h$ terms was not successful in this work, as the additional steps required to define $h$ in one of the above manners increased the computational time 
significantly. The interaction of the two particle types at the gas-water interface was also found to be prohibitive in implementing a smoothing length defined on a set number of interacting neighbours.

\subsubsection{Computational Time}

To prevent unnecessarily small time-steps whilst maintaining stability within the integration, the fixed time-step in the code has been replaced with an adaptive time-step based on the Courant-Friedrichs-Levy (CFL) condition that also accounts for viscous dissipation given by [55]:

$$
\Delta t=\min \left(\frac{h_{i}}{c_{i}+0.6\left(\alpha_{\pi} c_{i}+\beta_{\pi} \max \left(\phi_{i j}\right)\right.}\right)
$$

The method used to search for neighbouring particles has a large influence on the computational time of the simulation. The simplest method is direct search, where every particle is compared to every other particle to determine the influence one has on the other. Alternative search methods include grid based searching and hierarchical tree and bucket methods. The Barnes-Hut tree search algorithm [3] was combined with SPH to form the TREESPH code of Hernquist and Katz [31], and is ideal for use with distributed memory parallel supercomputers [21, 73]. This method reduces search time from $\mathrm{O}\left(\mathrm{N}^{2}\right)$ (for the direct search method) to $\mathrm{O}(\mathrm{NlnN})$. Another tree search structure which similarly reduces the search time to $\mathrm{O}(\operatorname{lnN})$ is the kd-tree. In this work, the kd-tree algorithm of Kennel [39] has been integrated into the code and has reduced the computational time accordingly.

Even considering the tree search method, for a large number of particles the numerous interactions and calculations can result in large computational times. Work has been done in SPH modelling to reduce this time by parallelising the codes. Goozée and Jacobs [30] improved the run time of their SPH code by a factor of 3 on four processors using OpenMP. Message Passing Interface (MPI) is also used for parallelising code, allowing distributed memory computers to be used; however, it is not as efficient as OpenMP for smaller simulations. For this work OpenMP was used to parallelise the code, improving the speed only by $100 \%$, which is much less than the ideal $400 \%$ with four threads. 


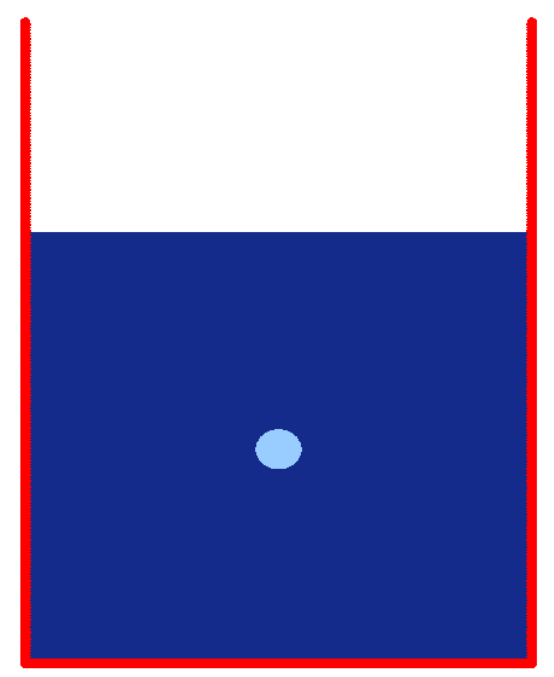

Figure 2.1: Configuration of SPH simulation of a gas bubble with radius $0.01 \mathrm{~m}$ in an open top water tank with dimensions $0.3 \mathrm{~m}$ (width) $\times 0.45 \mathrm{~m}$ (height) (water depth $0.3 \mathrm{~m}$ ). The light blue represents the gas bubble, the dark blue represents the water and the red represents the boundaries. The bubble is represented disproportionately large within the domain. These dimensions were chosen to reduce the overall number of particles and still maintain a viable particle resolution in the bubble.

\subsection{Code Development Results and Discussion}

\subsubsection{Configuration}

As noted above, previous simulations of underwater explosion bubbles have considered the process totally enclosed by solid boundaries [31, 58]. The expansion of the bubble within solid boundaries is not considered to be realistic, as it requires un-physical compression of the water and results in a bubble that pulses due to reflection of shock waves, rather than the pressure difference between the gas and water. For this reason, the simulations in the present work include a free surface. Reflections of shock waves will still interact with the bubble; however, the free surface allows the water level to rise and accommodate the increasing bubble volume. To prevent incomplete kernels at the boundary causing unnatural pressure differentials, the free surface water pressure is modelled at 0 bar (gauge) [64], and atmospheric pressure, $p_{a}$, is accounted for in the equation of state for the gas to ensure the correct pressure difference between the water and bubble. Equation 2.11 is replaced with:

$$
p=(k-1) e_{i} \rho_{i}-p_{a}
$$


The initial configuration is shown in Figure 2.1. In this case, the water particles are contained in a tank $0.3 \mathrm{~m}$ (width) $\times 0.45 \mathrm{~m}$ (height). The water is $0.3 \mathrm{~m}$ deep, and the bubble is centrally located and has a radius of $10 \mathrm{~mm}$. This represents a bubble that is disproportionately large for the domain; however, these dimensions were chosen to reduce the number of particles while maintaining a viable particle resolution in the bubble itself. The total number of water and gas particles is $10^{6}$, resulting in a particle spacing of $1.2 \mathrm{~mm}$. The tank boundary is composed of stationary virtual particles, as discussed in Section 2.2.2, at the same spacing as the real particles. The threedimensional configuration is similar, with a sphere in an open top box.

The spherical nature of the bubble defines the physics of the problem as inherently three-dimensional. In order to reduce the computational power required, and to allow easier visualisation of the particle interaction during development, the majority of the simulations were two-dimensional. This results in the bubble behaving as an infinitely long cylinder. While this affects the rate of change in gas density of the bubble, the simulation properties of the particle interaction, in particular the issues encountered at the gas-water interface (discussed in Section 2.3.4) do not change. For this reason the two-dimensional case was considered a viable intermediate step in developing the code, and only two-dimensional views will be shown in the figures.

\begin{tabular}{lc}
\hline Initial bubble pressure & $128 \mathrm{bar}$ \\
Initial bubble radius & $0.01 \mathrm{~m}$ \\
Particles & $10^{6}$ \\
$\alpha_{\pi}$ & 0.5 \\
$\beta_{\pi}$ & 0.5 \\
$\varepsilon$ & 0.5 \\
$c$ (numerical speed of sound in water) & $500 \mathrm{~ms}^{-1}$ \\
Density re-initialisation & every 30 time-steps \\
\hline
\end{tabular}

Table 2.1: SPH parameters for shock wave propagation simulation.

\subsubsection{Basic Results}

The configuration described above has been used to model the pressurised gas bubble in a contained body of water with a free surface. The parameters used in the simulation are given in Table 2.1. The speed of sound used in the simulation directly affects the simulation time-step and, therefore, the overall run time. A numerical speed of sound of $500 \mathrm{~ms}^{-1}$ has been used to reduce the run time while developing the dynamics of the simulation. 


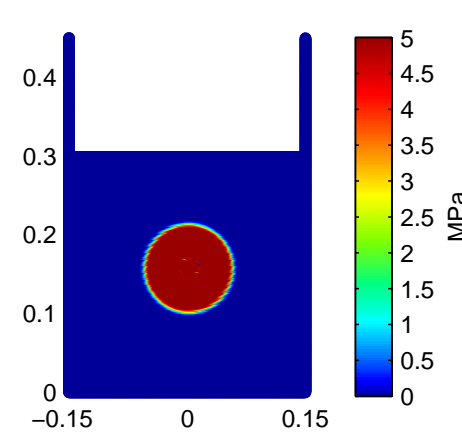

(a) $t=0.088 \mathrm{~ms}$

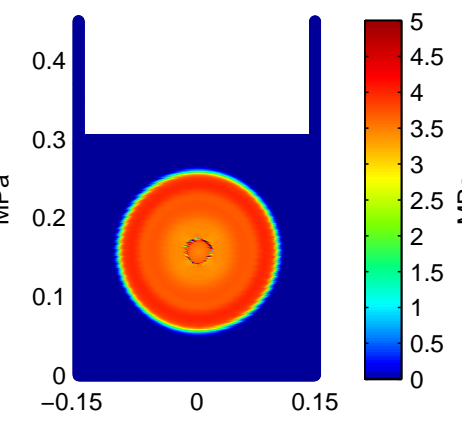

(b) $t=0.177 \mathrm{~ms}$

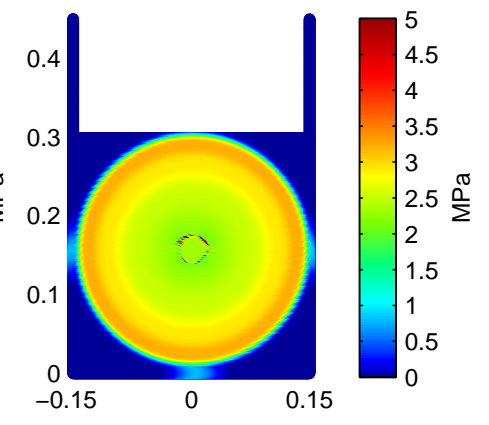

(c) $t=0.265 \mathrm{~ms}$

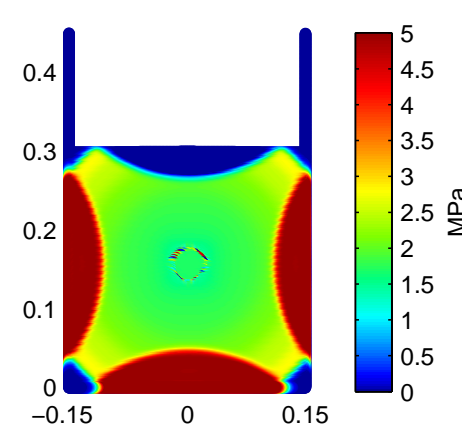

(d) $t=0.353 \mathrm{~ms}$

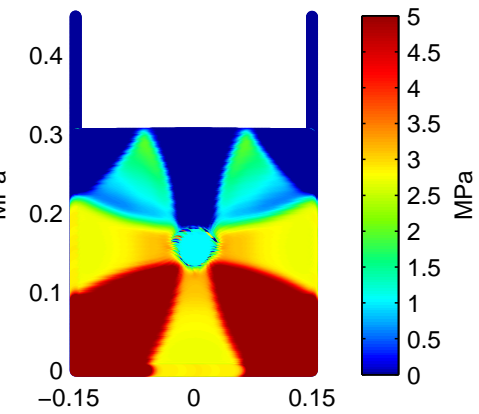

(e) $t=0.530 \mathrm{~ms}$

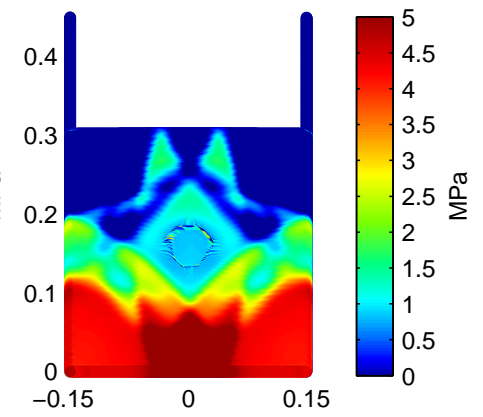

(f) $t=0.707 \mathrm{~ms}$

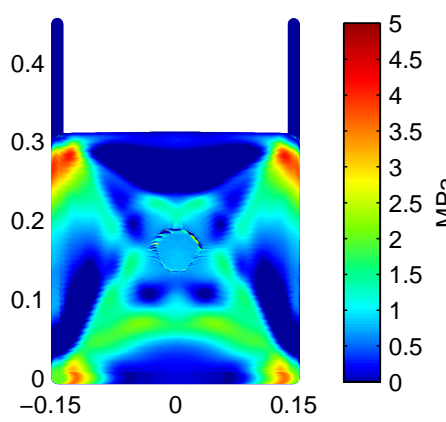

(g) $t=0.885 \mathrm{~ms}$

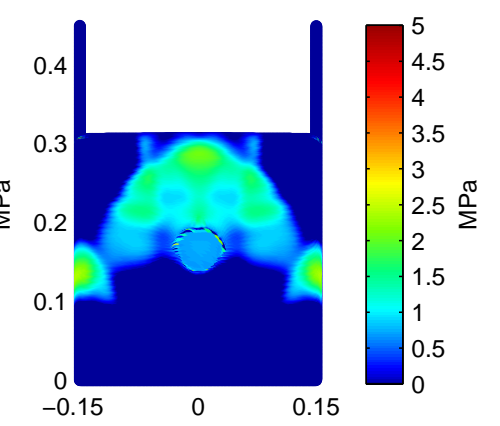

(h) $t=1.063 \mathrm{~ms}$

Figure 2.2: Pressure contour plots from an SPH simulation of a highly pressurised gas bubble with initial radius $0.01 \mathrm{~m}$ in an open top water tank with dimensions $0.3 \mathrm{~m}$ (width) $\times 0.45$ $\mathrm{m}$ (height) (water depth $0.3 \mathrm{~m}$ ). The bubble expands and generates an initial shock wave, as shown in images a $-c$, which reflects off the boundaries (image d). The bubble continues to expand (images $\mathrm{e}-\mathrm{g}$ ) until the size difference between the gas and water particles prevents accurate interaction (image h). A detailed view of image $h$ is shown in Figure 2.3. 
Figure 2.2 depicts the initial results of the simulation. The radiation of the initial shock at the start of the simulation $(t=0.088 \mathrm{~ms})$ is representative of the airgun firing or explosive detonation and is shown in Figure 2.2a. The shock propagates through the water at the speed of sound, in this case $500 \mathrm{~ms}^{-1}$, (Figures 2.2b, c) before reflecting from the solid boundaries and free surface (Figure $2.2 \mathrm{~d}$ ). The pressure reflections in the tank subside (Figures $2.2 \mathrm{e}-\mathrm{h}$ ) and the bubble continues to grow. At this point, the spacing of the gas particles becomes much larger than that of the water particles, as shown in Figure 2.3. This results in errors in the smoothing kernel, and thus the density calculation, due to the difference in particle sizes (discussed further in Section 2.3.4). The errors in the density calculation impact the smoothing length update of the gas particles, which eventually prevents their interaction. The bubble continues to grow due to the outward velocity of the particles; however, as the particles are not interacting, their density does not change and therefore neither does the pressure of the bubble. At this point the simulation is stopped.

\subsubsection{Limitations in Domain Size}

When simulating underwater explosions with $\mathrm{SPH}$, the outgoing pressure pulse from the expansion of the bubble reflects from the boundaries and can cause the bubble to collapse prematurely or significantly alter its dynamics. Different boundary conditions have been investigated using SPH, including Lastiwka et al. [48], who investigated permeable boundaries which are associated with either inflow or outflow zones. Simulations were successfully conducted with open domains for one- and two-dimensional flow around a cylinder. Gong [29] modelled a sponge layer on the boundary which was used in a wedge water entry simulation to reduce the shock wave reflections. Swegle and Attaway [79] overcame shock wave reflections in their underwater explosion simulations by placing the boundaries far enough away that the reflections did not impact on the first bubble period.

In addition to preventing shock wave reflections, the simulation of a pulsing bubble also requires the domain to be large enough to contain the fully expanded bubble, which may be up to $7-8$ times the initial radius, plus the surrounding water. Additionally, the domain must contain a very high particle density to accurately model the small initial bubble. For a three-dimensional simulation with a few hundred particles in the bubble, a domain size that allows only 1 bubble diameter between the expanded bubble and the wall would require $2.8 \times 10^{9}$ particles. Even so, containing the bubble in such a small container would dramatically alter the dynamics. One expansion and collapse cycle may take $6 \times 10^{4}$ time-steps. The computational power required for this size domain was unachievable. 


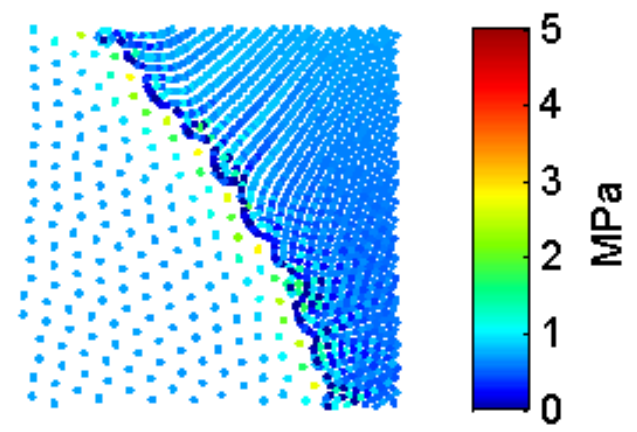

Figure 2.3: Detailed view of Figure $2.2 \mathrm{~h}$ showing particle density at the gas-water interface. The particle density of the gas bubble is much larger than the particle density of the surrounding water. This prevents the particles of the different fluids from interacting accurately, and ultimately leads to failure of the simulation.

\subsubsection{Gas-Water Interface}

The basic SPH method assumes an even particle density (i.e., spacing between particles) and uniform particle mass in order to achieve a total kernel value approximately equal to 1 . At the interface between two fluids with greatly differing densities (for example, air and water), if a consistent particle density is maintained, the different fluid densities result in different particle masses. This skews the contribution of particles of different fluids to the particle summation, and for density differences greater than a factor of 100 , errors at the interface are likely to cause simulation failure. Colagrossi and Landrini [10] overcome this by using a volume based particle summation in place of Equation 2.7 given by:

$$
\frac{\mathrm{d} \rho_{i}}{\mathrm{~d} t}=\rho_{i} \sum_{j=1}^{N} \frac{m_{j}}{\rho_{j}} \mathbf{v}_{i j} \cdot \nabla_{i} W_{i j}
$$

The form of Equations 2.5 and 2.6 are variationally consistent with this approach [10]. In an underwater explosion or seismic airgun firing, as the gas expands the particle density of the gas rapidly becomes much lower than the particle density of the surrounding water and errors are incurred at the fluid interface. The smoothing length of the smaller water particles is generally too small to encompass a sufficient number (if any) of the larger gas particles, as illustrated in Figure 2.4, and increasing the smoothing length only serves to over smooth the water properties. Any gas particles that are included in the water particle's summation make too large a contribution to the water particle's properties, giving a kernel value greater than 1 and therefore an erroneous density value. 


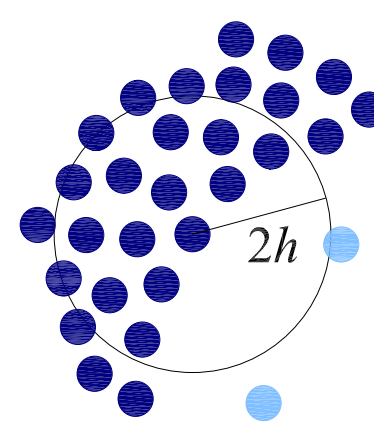

Figure 2.4: The interaction of particles with significantly differing particle densities. The smoothing length of the dark blue water particles does not include the larger light blue gas particles, which results in errors in the particle approximations.

A possible solution to this problem is particle splitting. Kitsionas and Whitworth [41] used particle splitting to increase local resolution when simulating self-gravitating collapses. Two methods for splitting the particles were investigated, one method split particles at defined simulation times, and the other split particles based on resolution. In both cases, the parent particle was split into 13 children. Lastiwka et al. [47] present a general method to add and remove particles based on any pre-defined criteria. When the particles are added or removed, fluid volume and particle mass are redistributed in the local area. This method improved global density error in capturing the shock in a one-dimensional Riemann shock tube simulation. Hong et al. [33] split and merged particles based on both pre-defined distance from the fluid surface and Reynolds number to improve particle resolution at the surface. Single particles were split into two or several particles were merged together at the time the particles crossed into a new layer. Upper and lower limits were set for particle size.

When particle splitting was implemented for the expanding gas particles in this work, errors accumulated rapidly causing the simulation to fail within a few time-steps. Difficulties were encountered in maintaining both conservation of momentum and the outward radial growth direction of the bubble. The additional calculations also increased the required computational power and simulations quickly became impractically large. 


\subsection{Conclusion}

An attempt was made to use SPH to model the bubble dynamics of a highly pressurised gas bubble in water. An SPH model was updated to model the bubble in an open top tank and to include a tree search method for particle neighbour searching, and a method for improving interactions between particles of different fluids. The implementation of grad- $h$ terms was investigated; however, it was un-successful. Limitations were found in the computational power and time required to model an appropriately sized domain that was large enough to contain the fully expanded bubble and provide sufficient particle resolution. These limitations also impacted the ability to incorporate other methods for improving the simulation. The drastic difference in the particle density of the water and the gas as the bubble expands lead to significant errors at the gas-water interface that ultimately prevented a successful simulation. Particle splitting of the gas particles is suggested as a possible solution to overcome this problem. 


\title{
Modelling of Seismic Airgun Bubble Dynamics and Pressure Field using the Gilmore Equation with Additional Damping Factors
}

An abbreviated version of this chapter was presented at the (peer-reviewed) $18^{\text {th }}$ Australasian Fluid Mechanics Conference, 3-7 December 2012. The chapter as included here has been submitted for publication with Journal of Ocean Engineering. The conference paper is included in Appendix A. The contributing authors are: K.L. de Graaf, I. Penesis and P.A. Brandner.

The citation for the conference paper is:

de Graaf, K.L., Penesis, I., Brandner, P.A., Comparison of the Rayleigh-Plesset and Gilmore Equations and Additional Aspects for the Modelling of Seismic Airgun Bubble Dynamics, Proceedings of the $18^{\text {th }}$ Australasian Fluid Mechanics Conference, 3-7 December, Launceston Tasmania, Australia

\begin{abstract}
Seismic airguns are commonly used in geophysical exploration. More recently, they are also being used as an alternative to underwater explosions for the shock testing of defence vessels. The study of the dynamics of the bubble produced by a seismic airgun is beneficial in understanding the resultant pressure field and shockwave. The RayleighPlesset and Gilmore equations for modelling spherical bubble dynamics are compared for the expansion of an initially highly pressurised gas bubble. The relationship between initial gas pressure and both the first maximum bubble radius and the first period of oscillation are presented. The separate contributions due to the presence of the airgun
\end{abstract}


body, mass throttling, effective viscosity and heat diffusion to the first maximum radius and period are modelled and discussed. The effects of evaporation and condensation at the bubble wall are also considered. Gilmore's model is used to predict the radiated pressure wave of the new bubble model. The results are in good agreement with measured data collected from full-scale airgun trials.

\subsection{Introduction}

The Royal Australian Navy is currently investigating the feasibility and advantages of employing seismic airguns for shock testing naval craft. Shock testing with seismic airguns, rather than high explosives, is less expensive, safer, and more environmentally friendly. To perform shock testing effectively, an array of airguns must be used and the interactions between the bubbles can alter the pressure fields produced. Several methods exist for calculating the interactions between bubbles in an array [51, 83], but all rely on a basic understanding of the parameters affecting a single airgun bubble and the pressure field and shockwave produced.

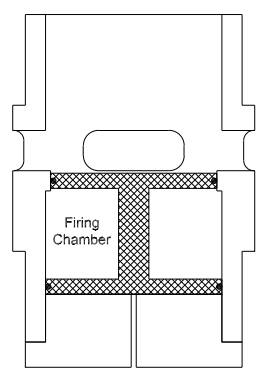

(a)

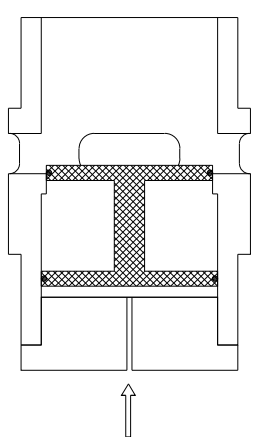

(b)

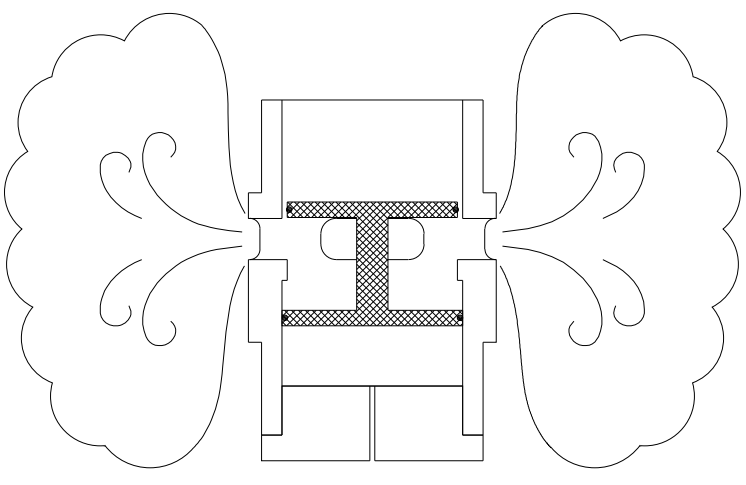

(c)

Figure 3.1: Illustration of airgun firing in three stages: (a) pressurised chamber under equilibrium; (b) equalisation of pressure across lower flange to fire shuttle and (c) release of air through ports to form bubble.

The underlying principle of an airgun relies on a firing chamber, filled with compressed gas, which is held shut via a pressure difference across the two ends of a shuttle. The lower flange of the shuttle in Figure 3.1a is larger than the upper flange. When the pressure difference across one end of the shuttle (in this case, the lower flange) is equalised (Figure $3.1 b)$, the shuttle can open and the compressed air in the firing chamber exhausts through several 'ports' in the body of the airgun, and forms the bubble. The gun is usually reset through the use of a second chamber of air - not shown here. 
The Gilmore equation for bubble dynamics is commonly used as the underlying basis for seismic airgun bubbles and underwater explosions. Comparisons of this equation with other bubble models, including the well known Rayleigh-Plesset equation, exist; however, they consider a bubble's collapse from its maximum radius rather than expansion from its minimum radius. In modelling seismic airgun bubbles, it is more practical to consider the initial bubble pressure and radius, rather than the conditions at the first maximum. The present work compares the Gilmore equation to the Rayleigh-Plesset equation to confirm the use of the Gilmore equation as the basic bubble model.

Several contributions have been made to improve the numerical modelling of individual seismic airgun bubbles by considering additional factors to the basic bubble dynamics. Ziolkowski [89] used Gilmore's equation and found a polytropic index of 1.13 gave good results for the first period of oscillation; this value was also obtained by Dragoset [20] for a range of gun sizes. Shulze-Gatterman [75] emphasised the effect of the actual airgun body on the period of oscillation. Safar [74] compared the equation of a bubble to an electrical circuit and developed a model for the rise time, amplitude of the initial pulse, and period of the airgun. Johnston [37] and Dragoset [20] considered the effect of the shuttle motion and choked flow rate on the chamber pressure, with Dragoset allowing for the actual port size. Ziolkowski [90] proposed that heat transfer occurs through the latent heat absorbed and released by evaporation and condensation at the bubble wall. This concept is repeated by Langhammer and Landrø [43]. Laws et al. [51] consider mass transfer due to evaporation and condensation, conductive heat diffusion, flow throttling and an 'effective viscosity' induced by the turbulent nature of the bubble. It is claimed that this turbulent nature also has an amplifying effect on the heat transfer across the bubble wall. Li et al [53] includes the effect of mass throttling (but not choked flow) through ports, the airgun body, heat transfer and hydrostatic pressure changes as the bubble rises through the water.

There appears to be no work that considers all of these parameters together and provides values for coefficients with a summary of the impact of each parameter on the bubble behaviour. In this work, the Gilmore equation is used as the basic bubble model and the individual and combined effects of the presence of the airgun body, mass throttling, effective viscosity, heat diffusion and condensation and evaporation are considered. Preliminary results using this approach have been presented by de Graaf [16]. The present work provides more detailed results, including a prediction of the radiated pressure wave using Gilmore's theory, and makes comparison with experimental data. 


\subsection{Comparison of Rayleigh-Plesset and Gilmore Equations}

The Rayleigh-Plesset equation describes the motion of a spherical bubble in an incompressible liquid [23]. When considering bubble velocities of an appreciable order of magnitude compared with the speed of sound in water, compressibility of the liquid cannot be ignored. The Gilmore equation includes second-order compressibility terms, accounting for the loss of bubble energy due to the radiated pressure waves [27]. Both equations are commonly used to model bubble dynamics, with the Gilmore equation often used in underwater explosion applications. The Rayleigh-Plesset equation is given by:

$$
\rho\left[R \ddot{R}+\frac{3}{2} \dot{R}^{2}\right]=p_{0}\left(\frac{R_{0}}{R}\right)^{3 k}+p_{v}-\frac{2 S}{R}-4 \mu \frac{\dot{R}}{R}-p_{\infty}(t)
$$

The Gilmore equation is given by:

$$
R \ddot{R}\left(1-\frac{\dot{R}}{c}\right)+\frac{3}{2} \dot{R}^{2}\left(1-\frac{\dot{R}}{3 c}\right)=H\left(1+\frac{\dot{R}}{c}\right)+\frac{R \dot{H}}{c}\left(1-\frac{\dot{R}}{c}\right)
$$

where

$$
\begin{gathered}
H=n\left(\frac{p_{\infty}+B}{(n-1) \rho}\right)\left[\left(\frac{p+B}{p_{\infty}+B}\right)^{\frac{n-1}{n}}-1\right] \\
c=c_{\infty}\left[\left(\frac{p+B}{p_{\infty}+B}\right)^{\frac{n-1}{2 n}}\right] \\
p=p_{0}\left(\frac{R_{0}}{R}\right)^{3 k}+p_{v}-\frac{2 S}{R}-4 \mu \frac{\dot{R}}{R} \\
c_{\infty}=\sqrt{n\left(\frac{p_{\infty}+B}{\rho}\right)}
\end{gathered}
$$

$R$ is the bubble radius, $\rho$ is the water density, $p_{v}$ is the water vapour pressure, $p_{\infty}$ is the ambient water pressure, $p_{0}$ is the initial non-condensable gas pressure, $R_{0}$ is the initial bubble radius, $k$ is the polytropic index of the bubble gas, which varies between 1 (isothermal) and 1.4 (adiabatic) - taken as 1.4 in this work where required, $S$ is the surface tension, $\mu$ is the dynamic viscosity of water, $H$ is the enthalpy difference between the liquid at pressure $p$ and $p_{\infty}$, and $c_{\infty}$ is the speed of sound in water at an infinite distance from the bubble. $B$ and $n$ are constants used to calculate the local speed of sound of in water, $c$, and enthalpy. At the bubble surface, it is assumed that the pressure of the bubble gas is equal to the water pressure. Gilmore [27] gives $B=3000 \mathrm{~atm}$ and $n=7$. Overdots represent differentiation with respect to time. 
The equations are solved for $R(t)$ using the classical fourth order Runge-Kutta method. An adaptive time-step is not required due to the large non-condensable gas content of the bubble which prevents the nearly infinitely high bubble wall velocities that can cause the more predominantly vapour-filled cavitation bubble simulations to fail.

Vokurka [85] compared these two equations for bubble collapse and determined that for maximum radius vs. equilibrium radius ratios of greater than 2, the Gilmore equation produces superior results. Other comparisons made between these equations also model the bubble collapse assuming the bubble starts at its maximum radius and lowest pressure - representative of a newly formed cavitation bubble. In these comparisons, the collapse of a Gilmore bubble is fractionally (1.005) longer than the collapse of a Rayleigh bubble [23].

This work compares the Rayleigh-Plesset and Gilmore equations with the bubble initially at its smallest radius and highest pressure - as would be more appropriate when considering seismic airguns and underwater explosions. For both equations, the first maximum radius as a function of the initial pressure for the expanding bubbles is shown (Figure 3.2). The maximum radius, $R_{M}$, and initial pressure have been non-dimensionalised with respect to initial bubble radius and ambient water pressure respectively.

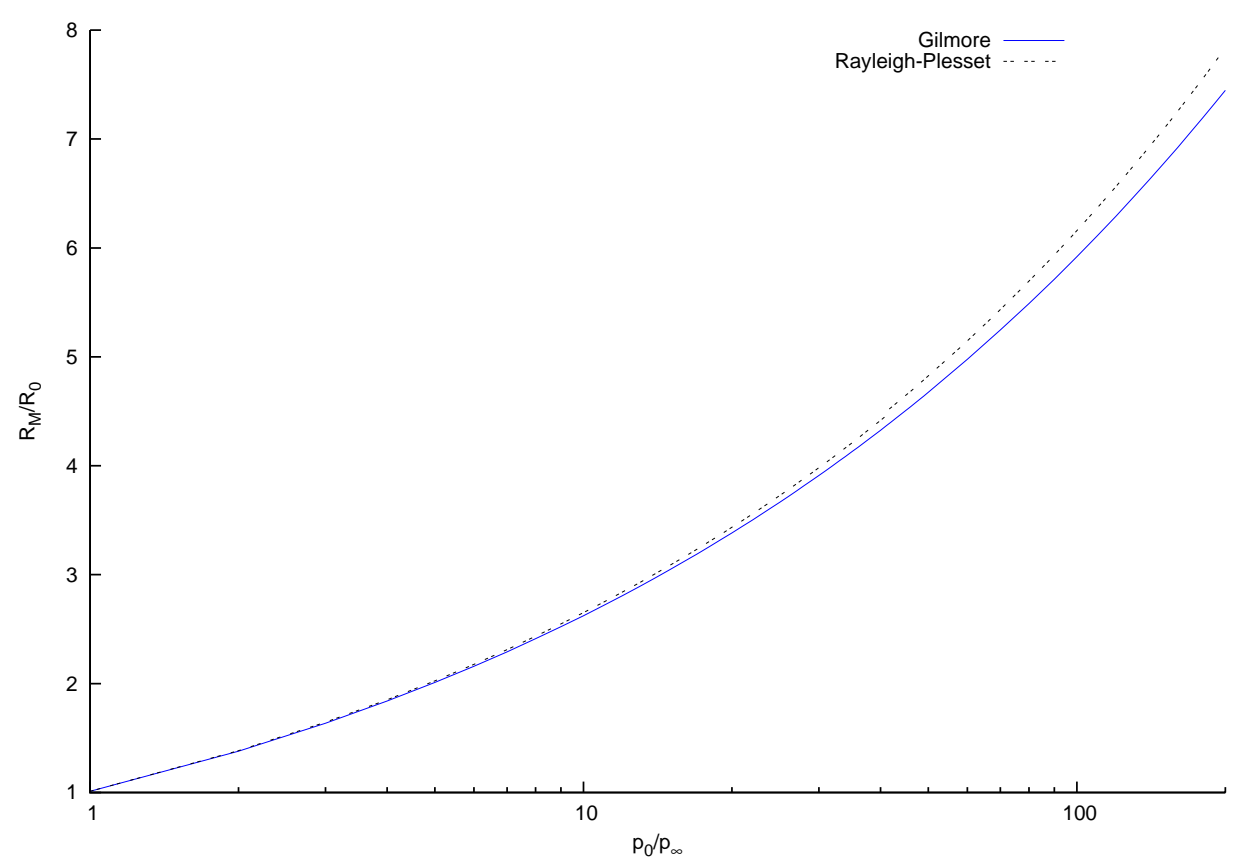

Figure 3.2: First maximum radius as a function of initial pressure predicted by RayleighPlesset and Gilmore equations with initial radius of $0.1 \mathrm{~m}$. Other variables are taken as: $p_{\infty}$ $=1 \times 10^{5} \mathrm{~Pa}, \rho=1000 \mathrm{~kg} / \mathrm{m}^{3}, p_{v}=1910 \mathrm{~Pa}, \mu=1 \times 10^{-3} \mathrm{~kg} / \mathrm{ms}$ and $S=0.074 \mathrm{~N} / \mathrm{m}$. 
When modelling a bubble initially at its minimum point through one expansion and collapse cycle, a smaller maximum bubble radius is predicted by the Gilmore equation, which leads to a shorter period when compared with the Rayleigh-Plesset solution (Figure 3.3). This study compares the Rayleigh-Plesset and Gilmore equations for use in

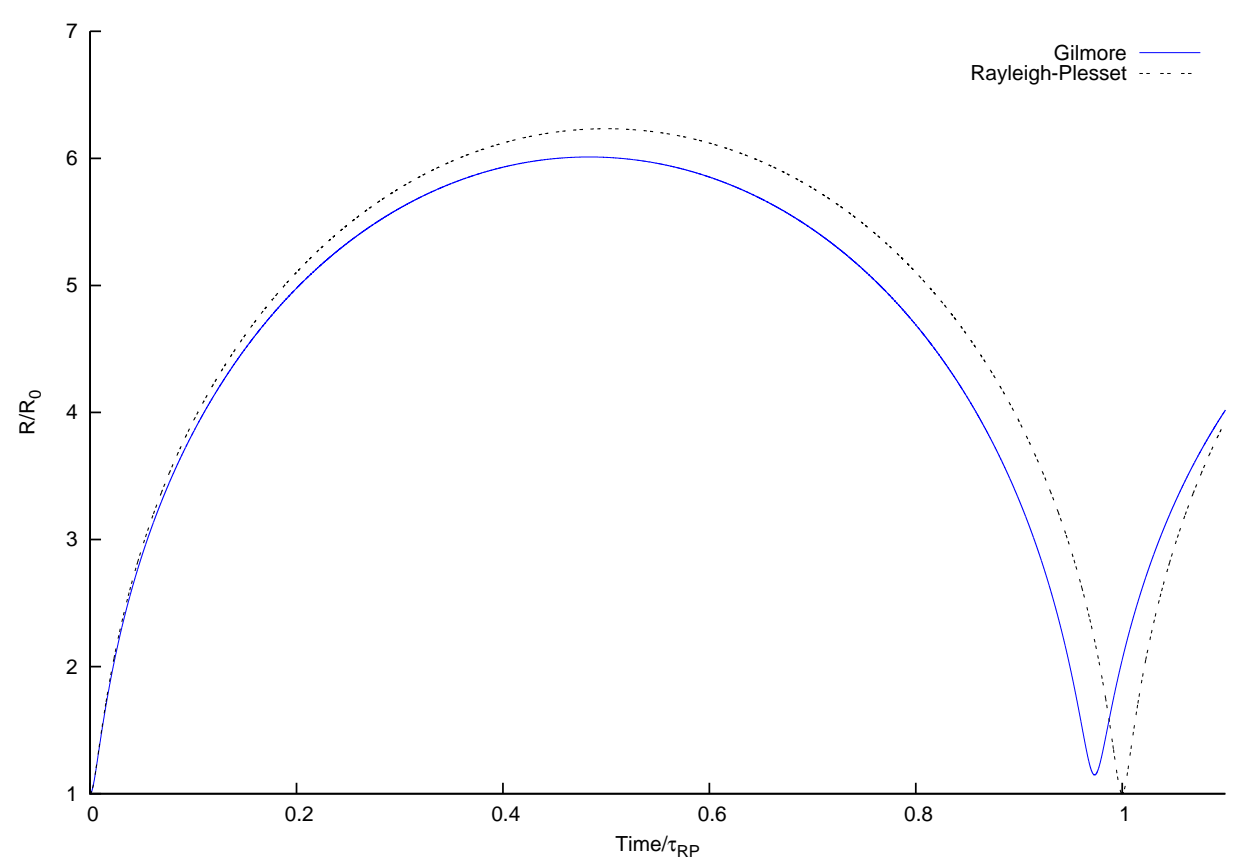

Figure 3.3: Comparison of predicted temporal variation of non-dimensional bubble radius from Rayleigh-Plesset and Gilmore equations for an initial pressure and radius of 100 bar and 0.1 $\mathrm{m}$, respectively. Other variables are taken as: $p_{\infty}=1 \times 10^{5} \mathrm{~Pa}, \rho=1000 \mathrm{~kg} / \mathrm{m}^{3}, p_{v}=1910$ $\mathrm{Pa}, \mu=1 \times 10^{-3} \mathrm{~kg} / \mathrm{ms}$ and $S=0.074 \mathrm{~N} / \mathrm{m}$. $\tau_{R P}$ is the collapse time for the Rayleigh-Plesset bubble.

modelling seismic airgun or underwater explosion type bubbles (initially at a minimum radius and maximum pressure) in contrast to modelling cavitation type bubbles (initially at maximum radius and minimum pressure) - the latter being the commonly published study. Further study shows that the surface tension and dynamic viscosity terms are negligible in both solutions due to the size of these explosion type bubbles. Noticeable damping from these two terms is evident in bubbles with an initial radius in the order of $100 \mathrm{\mu m}$. The equivalent initial radius of a full-scale airgun is in the order of 10-100 $\mathrm{mm}$. Removal of these terms reduces the Rayleigh-Plesset equation to what is known as simply the Rayleigh equation.

Figure 3.2 illustrates the increasing disparity between the two equations with increasing initial pressure. For seismic airguns, the initial pressure is generally at least an order of magnitude greater than the ambient water pressure; resulting in substantially different solutions between the Gilmore and Rayleigh equations. The Gilmore equation has been 
developed for modelling underwater explosions and contains the higher order terms to account for increased damping due to radiated pressure waves. All further predictions will use this as the base model. As surface tension is negligible for the size bubble considered here, it will be ignored; however, a dynamic viscosity term is required when calculating the effective viscosity and the thermal boundary layer, and will be included in all simulations for consistency and taken as $1 \times 10^{-3} \mathrm{~kg} / \mathrm{ms}$.

\subsection{Additional Aspects to the Gilmore Equation}

It is well noted $[51,53,75,90]$ that while the Gilmore equation models compressibility which leads to acoustic damping, additional damping parameters must also be present in the dynamics of an airgun bubble. Typically, a real airgun bubble will have lost its energy after only a few growth/collapse cycles. Predictions with the Gilmore equation result in oscillations that persist much longer. The additional factors identified here have been modelled using the parameters corresponding to Firing A of the experimental data; the initial bubble radius is taken as $0.127 \mathrm{~m}$, the initial pressure is 138 bar and ambient water pressure is 1.29 bar.

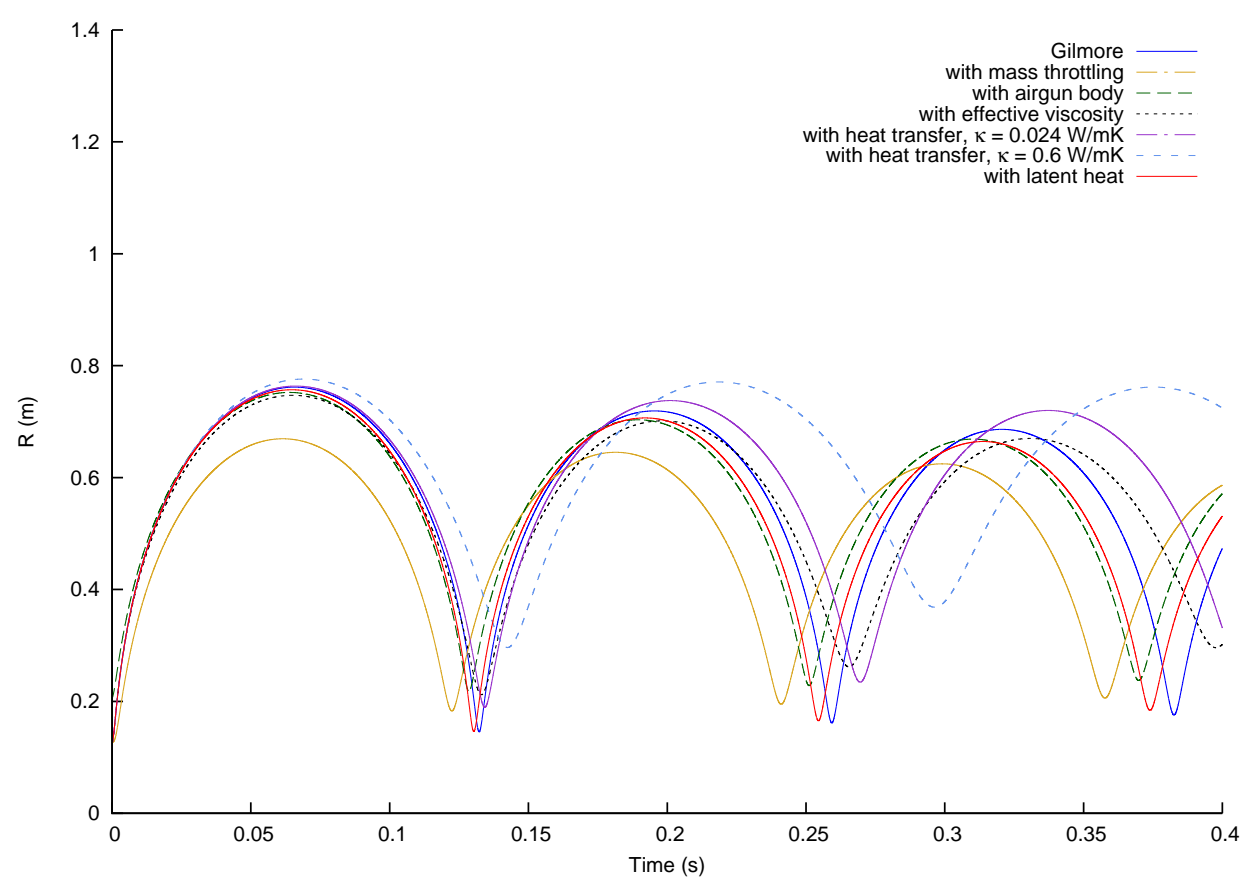

Figure 3.4: The effect of various aspects on the solution of the Gilmore equation. 
To allow the incorporation of terms for mass throttling and temperature effects, the ideal gas law pressure Equation (3.5) is replaced by:

$$
p=\frac{m R_{G} T}{V}+p_{v}
$$

where $m$ is the bubble gas mass, $R_{G}$ is the gas constant, $T$ is the bubble temperature and $V$ is the bubble volume.

When the gun is fired, the air expands through four 'ports' into the surrounding water. Depending on the gun design, the air may also pass through other internal constrictions before release. These orifices throttle the flow rate and considering the pressure differences involved, choked flow conditions can be assumed. The mass flow function is [88]:

$$
\dot{m}=A \sqrt{\frac{p_{G} m_{G}}{V_{G}} \frac{2 k}{k-1}\left[\left(\frac{p}{p_{G}}\right)^{\frac{2}{k}}-\left(\frac{p}{p_{G}}\right)^{\frac{k+1}{k}}\right]}
$$

where $p$ is limited to the sonic value, $p_{G}^{*}$ :

$$
p_{G}^{*}=p_{G}\left(\frac{2}{k+1}\right)^{\frac{k}{k-1}}
$$

$A$ is the orifice area, in this model taken as $0.0128 \mathrm{~m}^{2}$, and the subscript $G$ indicates the value inside the airgun chamber. The flow becomes unchoked when $p$ is greater than $p_{G}^{*}$. The mass throttling limits the initial maximum radius, subsequently reducing the pressure difference across the bubble wall and the intensity of the following bubble pulses. The reduced initial maximum radius also results in a comparatively shorter first period (Figure 3.4).

The presence of the airgun body at the bubble centre has been identified as a contributing factor to the bubble dynamics [75]. If the initial bubble radius is calculated from the volume of the airgun body plus the initial firing air volume, the volume of air in the bubble is:

$$
V=\frac{4}{3} \pi R^{3}-V_{A G}
$$

where $V_{A G}$ is the volume of the airgun body, in this model taken as $0.027 \mathrm{~m}^{3}$. This volume is used to calculate the bubble pressure, which in effect is increased at the maximum bubble radius as compared with the bubble pressure where no body is present. The reduced pressure difference between the bubble and the water reduces the intensity and velocities of the collapse; therefore, the first bubble minimum is larger, and the following oscillations less intense (Figure 3.4). 
Laws et al. [51] include an effective viscosity, $\mu_{e f f}$, to account for turbulent motion at the bubble wall which occurs at large Reynolds numbers. This increases the heat transfer across the bubble wall and the viscous damping of the bubble oscillation.

$$
\mu_{e f f}=\mu(1+K R e)
$$

$K$ is a constant, about 0.02. The Reynolds number, Re, is calculated based on the instantaneous bubble radius and velocity. The effect of increasing the viscosity has a small impact on the damping of the bubble (Figure 3.4). Note, the implementation of effective viscosity here considers only the increased viscous damping, and not the increased heat transfer across the bubble wall.

Li et al [53] identified that Ziolkowski's [89] model (essentially Gilmore's model with $k$ equal to 1.13) results in an equilibrium temperature well below that of the surrounding water. The inclusion of heat transfer in the model results in more realistic predictions of equilibrium temperatures. Laws et al. [51] give the thickness of the thermal boundary layer as:

$$
d=4 D \operatorname{Re}^{-\frac{3}{4}} \operatorname{Pr}^{-\frac{1}{2}}
$$

where $D$ is the bubble diameter and $\operatorname{Pr}$ is the Prandtl number for water. The rate of heat conduction across this layer is:

$$
\dot{Q}=\Delta T A \frac{\kappa}{d}
$$

where $\Delta T$ is the difference in temperature across the bubble wall, $A$ is the surface area of the bubble and $\kappa$ is the thermal conductivity of the interface. Ni et al [68] studied a range of $\kappa / d$ values from $2000-8000 \mathrm{~W} / \mathrm{m}^{2} \mathrm{~K}$ and found correspondence with specific experimental data for a value of $4000 \mathrm{~W} / \mathrm{m}^{2} \mathrm{~K}$. Herring [32] states that the flow of heat outward is a function of the thermal conductivity of the gas. The thermal boundary layer will extend across the bubble interface, complicating the value of the thermal conductivity. Here, values for $\kappa$ of $0.6 \mathrm{~W} / \mathrm{mK}$ for water and $0.024 \mathrm{~W} / \mathrm{mK}$ for air have been considered; $d$ is updated through the calculations but is in the order of $100 \mu \mathrm{m}$. The bubble has been assumed to have a uniform-temperature core with a thermal boundary layer at the surface. Due to turbulence, the area across which heat is conducted may be magnified - Laws et al. [51] used a factor of 10; however, in this example, the actual surface area is used.

For underwater explosions and spark or laser generated bubbles, the initial minimum bubble is hot and on expansion proceeds to be additionally cooled by the surrounding water. Conversely, the initial airgun bubble is assumed to be at ambient water 
temperature and as the bubble expands the drop in temperature is lessened by the surrounding water, allowing the bubble to reach a greater maximum radius. The following temperature increase upon collapse is also lessened by the heat transfer, arresting the collapse at a larger minimum radius. The magnitude of the following oscillations is likewise diminished (Figure 3.4). The bubble period is increased as a result of the larger maximum bubble radius and reduced velocity of the bubble motion. The variation of the bubble temperature with time is shown in Figure 3.5.

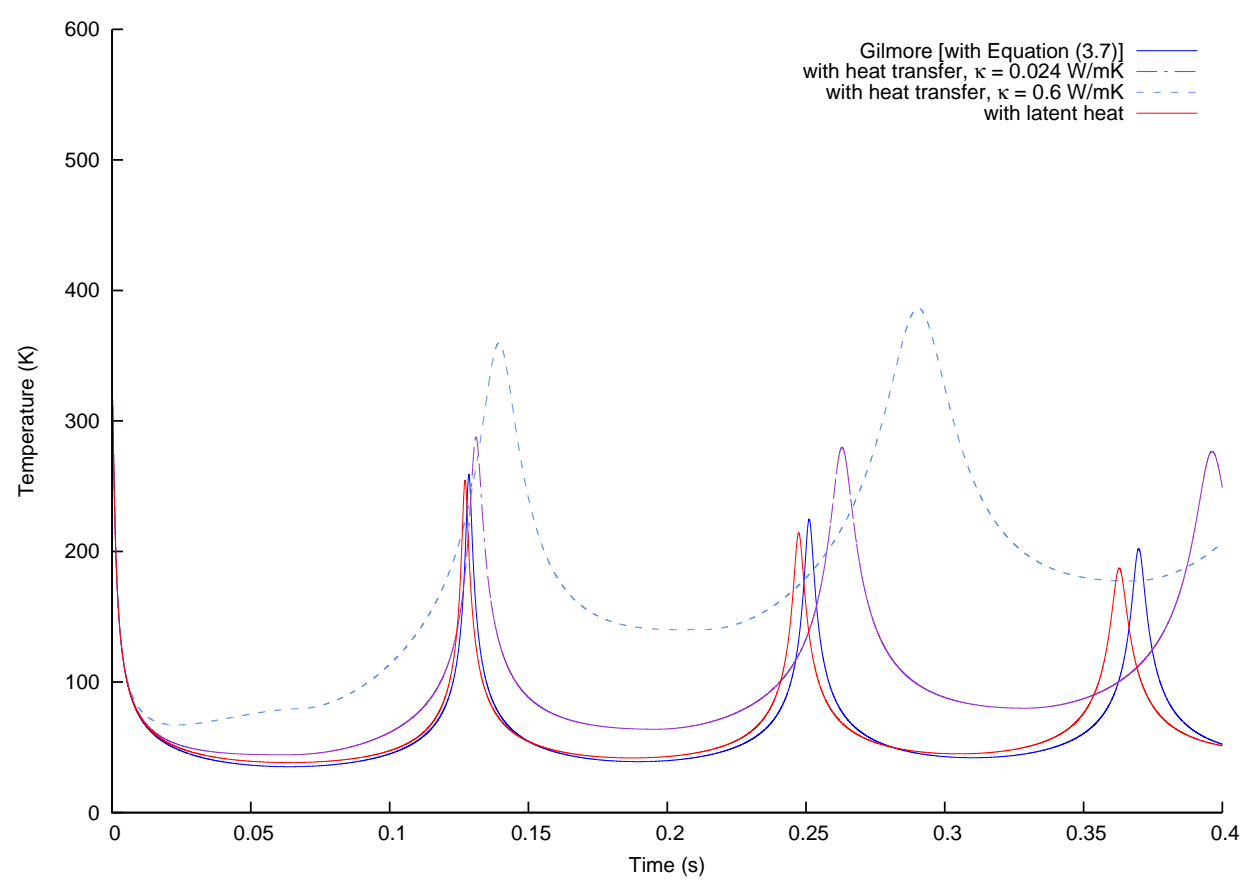

Figure 3.5: The effect of including conductive heat transfer and heat transfer through latent heat on the temperature predicted by the Gilmore equation. Ambient water temperature is $20^{\circ}$ Celsius.

In place of conductive heat transfer, Ziolkowski [90] proposed that the heat transfer takes place through latent heat absorbed/released by evaporation and condensation at the bubble wall. Fujikawa and Akamatsu [26] produced a detailed mathematical formulation to consider these effects and found that evaporation and condensation strongly influence microbubble dynamics. Simpler models have been used by Laws et al. [51] and Cook et al. [11]. The vapour mass transfer given by Fuijkawa and Akamatsu is:

$$
\dot{m}_{v}=\frac{\alpha_{M} A}{\sqrt{2 \pi R_{G}}}\left[\frac{p_{v}^{*}}{\sqrt{T_{w}}}-\Gamma \frac{p_{v}}{\sqrt{T}}\right]
$$

where $\alpha_{M}$ is the ratio of vapour molecules sticking to the phase interface and those impinging on it, about 0.04 [26], $A$ is the surface area of the bubble, $p_{v}^{*}$ is the equilibrium water vapour pressure [26] and $T_{w}$ is the temperature of the water. $\Gamma$ is a correction 
factor, assumed to be 1 for these calculations. The vapour pressure, $p_{v}$, is updated based on the vapour mass and limited to the saturation vapour pressure. The heat flow due to the vapour mass transfer is given by:

$$
\dot{Q}=\dot{m}_{v} L
$$

where $L$ is the latent heat of vaporization of water, taken as $2450 \mathrm{~kJ} / \mathrm{kg}$. The impact on the bubble wall dynamics due to movement of the bubble wall as a result of mass flow has been ignored, as the additional terms are assumed negligible [11]. Only the effect on bubble temperature and pressure has been considered. Modelling the temperature change due to evaporation and condensation has no appreciable difference on the final bubble temperature, and the mean temperature remains cooler than the surrounding water (Figure 3.5). There is little impact on the maximum radius and period of the bubble pulses (Figure 3.4). The results here differ from those of Fuijkawa and Akamatsu [26]; however, this may be attributed to the difference in size of the initial bubble radius.

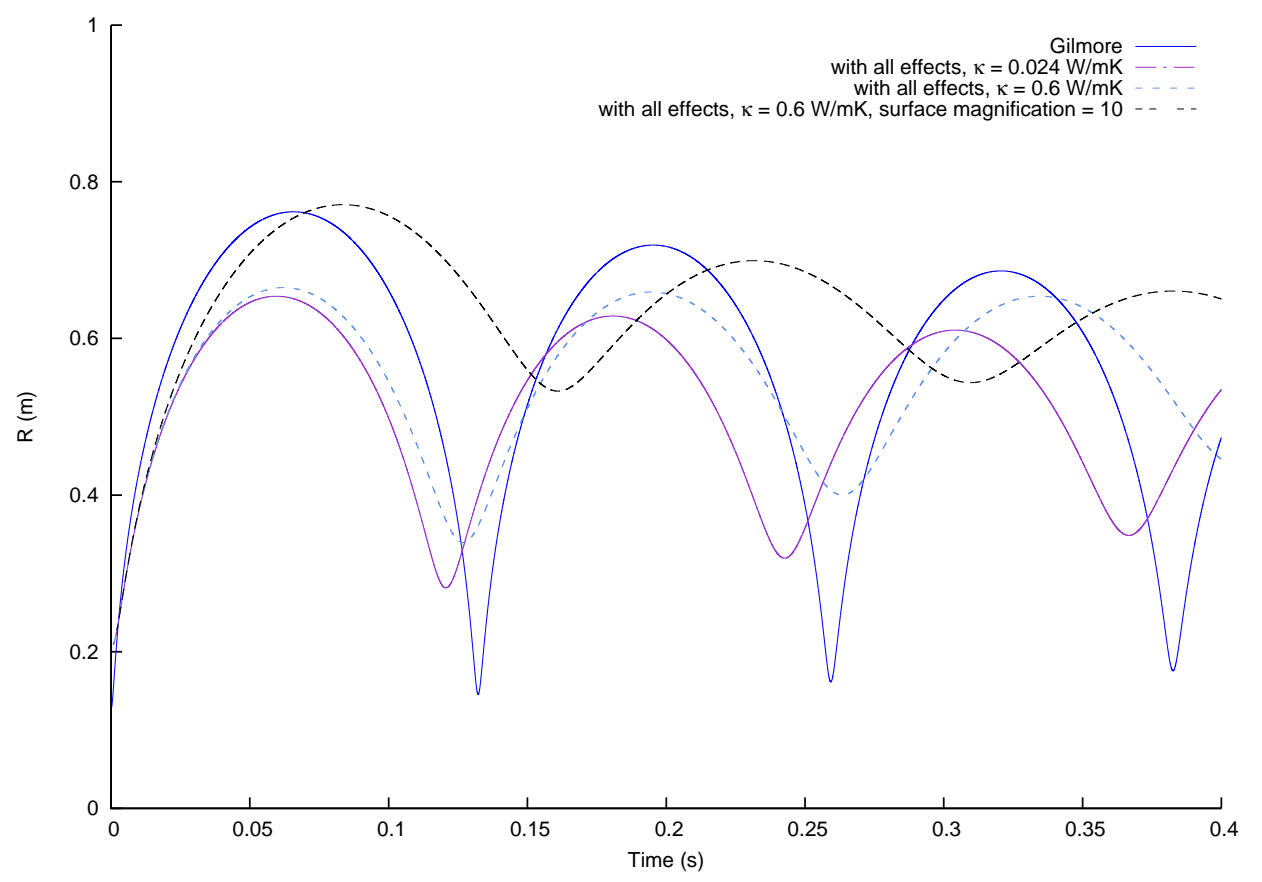

Figure 3.6: The combined effect of including all additional factors on the solution to the Gilmore equation.

Figure 3.6 presents the results of including all additional factors discussed here. Solutions have been calculated for both proposed limits of $\kappa$ with no increase in surface area (i.e. magnification factor of 1). Increasing the surface area in (3.13) further increases the bubble period and damping. A surface magnification factor of 10 is shown with $\kappa$ 
equal to $0.6 \mathrm{~W} / \mathrm{mK}$. The resultant bubble period corresponds well with the experimental waveform period (Figure 3.8a).

The additional aspects considered in this work have varying effect on the bubble radius and period predicted by the Gilmore equation. The inclusion of conductive heat transfer in the model has the single greatest effect on the bubble damping; however, the other terms, while independently having a smaller impact, do sum to have a net effect on the bubble that is worth considering.

\subsection{Pressure Field}

According to Gilmore, the pressure and liquid velocity fields surrounding the bubble are given by:

$$
\begin{gathered}
p_{l}-p_{\infty}=\rho_{\infty}\left(\frac{y}{r}-\frac{u^{2}}{2}\right)+\frac{\rho_{\infty}}{2 c_{\infty}^{2}}\left(\frac{y}{r}-\frac{u^{2}}{2}\right)^{2} \\
u_{l}=\frac{y}{c_{\infty} r}+\frac{K_{3} y^{2}}{c_{\infty}^{3} r^{2}}\left(1-\frac{y}{c_{\infty}^{2} r}+\frac{K_{3}^{2} y^{4}}{2 c_{\infty}^{8} r^{4}}\right) \\
t=t_{R}+\left(\frac{r-R}{c_{\infty}}\right)\left(1-\frac{\dot{R} R}{c_{\infty} r}\right) \\
y=R\left(H+\frac{\dot{R}^{2}}{2}\right) \\
K_{3}=\frac{c_{\infty}{ }^{3} R^{2} \dot{R}}{y^{2}}\left(1-\frac{\dot{R}^{2}}{2 c_{\infty}^{2}}\right)-\frac{c_{\infty}^{2} R}{y}\left(1-\frac{\dot{R}}{c_{\infty}}\right)
\end{gathered}
$$

where $p_{l}$ and $u_{l}$ are the pressure and velocity of the liquid at a point $r, t$ is the corresponding time co-ordinate to these values, $t_{R}$ is the time at which the characteristic started at the bubble wall $(r=R)$ and $y$ and $K_{3}$ are constants along any outgoing characteristic.

\begin{tabular}{lc}
\hline Firing Air Volume & $8521 \mathrm{~cm}^{3}$ \\
Equivalent Initial Radius & $0.12671 \mathrm{~m}$ \\
Airgun Body Volume & $0.0270 \mathrm{~m}^{3}$ \\
Throttling Area & $0.0128 \mathrm{~m}^{2}, 0.0074 \mathrm{~m}^{2 *}$ \\
\hline
\end{tabular}

Table 3.1: Parameters for the bubble model for the type 520 SERCEL airgun. ${ }^{*}$ The first area is based on the port size; a reduced area was estimated due to overprediction of the initial shock magnitude. 


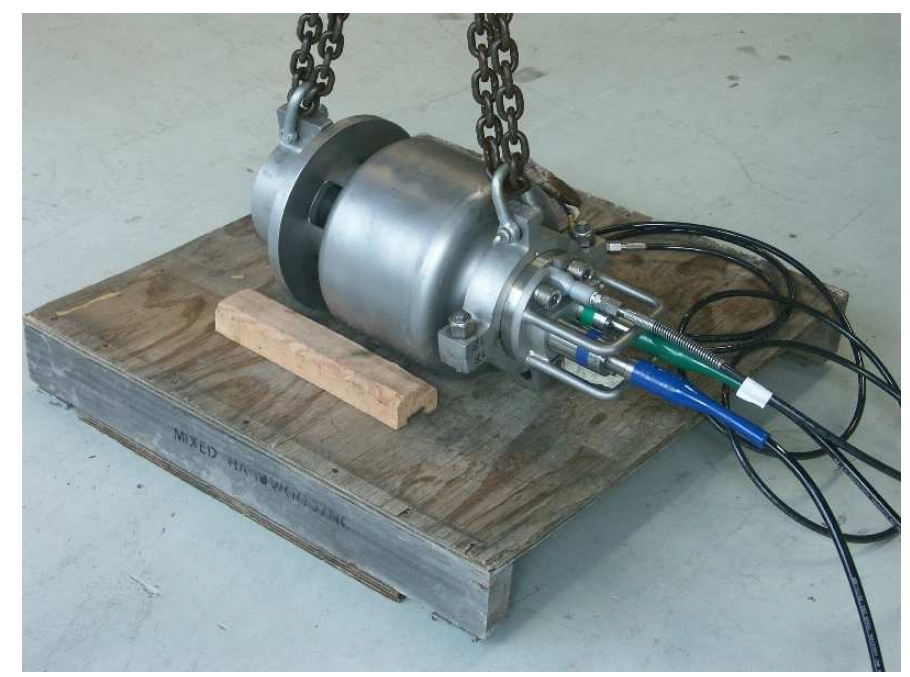

Figure 3.7: The type 520 SERCEL airgun owned by DSTO.

\subsection{Comparison of Pressure-Time History with Experimental Data}

Free-field pressure measurements for single airgun firings have been measured by the Australian Defence Science and Technology Organisation (DSTO) [12]. The results were obtained with a SERCEL type 520 airgun; airgun parameters for the bubble model are given in Table 3.1 .

Figure 3.8 compares the predicted pressure-time history of the current model (with $\kappa$ $=0.6 \mathrm{~W} / \mathrm{mK}$ and a surface area magnification factor of 10 [51]) with the experimental results. The initial pressure peak of an airgun is of short duration; to make it evident, the data has been plotted using a log time scale. The specific trial parameters for each comparison are summarised in Table 3.2.

With the throttling area based on the port size, the magnitude of the initial shock is overestimated by approximately $40 \%$. The internal design of the airgun causes complicated flow passages and complexities to develop during the release of the firing air. It is also possible that there are passages smaller than the ports inside the airgun which the air passes through. The air jet exiting the ports is also likely to be subject to a vena contracta. The limitations in modelling these phenomena and the resultant initial mass transfer significantly affect the prediction of the initial shock. To illustrate the effect of a smaller orifice size, Figure 3.8 also shows the predicted waveform using a reduced throttling area of $7.4 \times 10^{-3} \mathrm{~m}^{2}$. This results in a reduced predicted initial shock that gives good agreement with the measured waveform without significantly influencing the bubble period. No data is available for actual bubble size. 


\begin{tabular}{lc}
\hline \multicolumn{2}{l}{ Trial Configuration } \\
\hline \hline Firing A \\
\hline Firing Air Pressure & $138 \mathrm{bar}$ \\
Airgun Depth & $3 \mathrm{~m}$ \\
Pressure Gauge Depth & $3 \mathrm{~m}$ \\
Horizontal Standoff & $2.22 \mathrm{~m}$ \\
\hline Firing B & \\
\hline Firing Air Pressure & $207 \mathrm{bar}$ \\
Airgun Depth & $3 \mathrm{~m}$ \\
Pressure Gauge Depth & $3 \mathrm{~m}$ \\
Horizontal Standoff & $2.22 \mathrm{~m}$ \\
\hline Firing C & \\
\hline Firing Air Pressure & $172 \mathrm{bar}$ \\
Airgun Depth & $5 \mathrm{~m}$ \\
Pressure Gauge Depth & $5 \mathrm{~m}$ \\
Horizontal Standoff & $2.22 \mathrm{~m}$ \\
\hline
\end{tabular}

Table 3.2: Configuration parameters for airgun firings.

Comparison of the radius predicted by the present bubble model with experimental results will be the subject of future work. A scale model airgun and testing facility has been developed for controlled analysis of the bubble physics and pressure field.

\subsection{Conclusion}

Various models have been studied that could account for differences in airgun bubble dynamics between theoretical predictions and experimental observations. From the present study, the most likely primary cause of additional damping to the seismic airgun bubble is heat transfer between the water and the air. This is potentially enhanced by an increase in bubble surface area due to turbulent motion near the bubble wall. From comparison with experimental data, the increase in surface area is in the order of a factor of 10, which is in agreement with previous findings. Mass throttling as the air is released impacts the first maximum radius of the bubble and the magnitude of the initial shock wave significantly. The Gilmore pressure model has been used to predict the pressure-time history based on bubble motion from the improved model. The pressure waveform and bubble period results have been compared with experimental data with good agreement. 


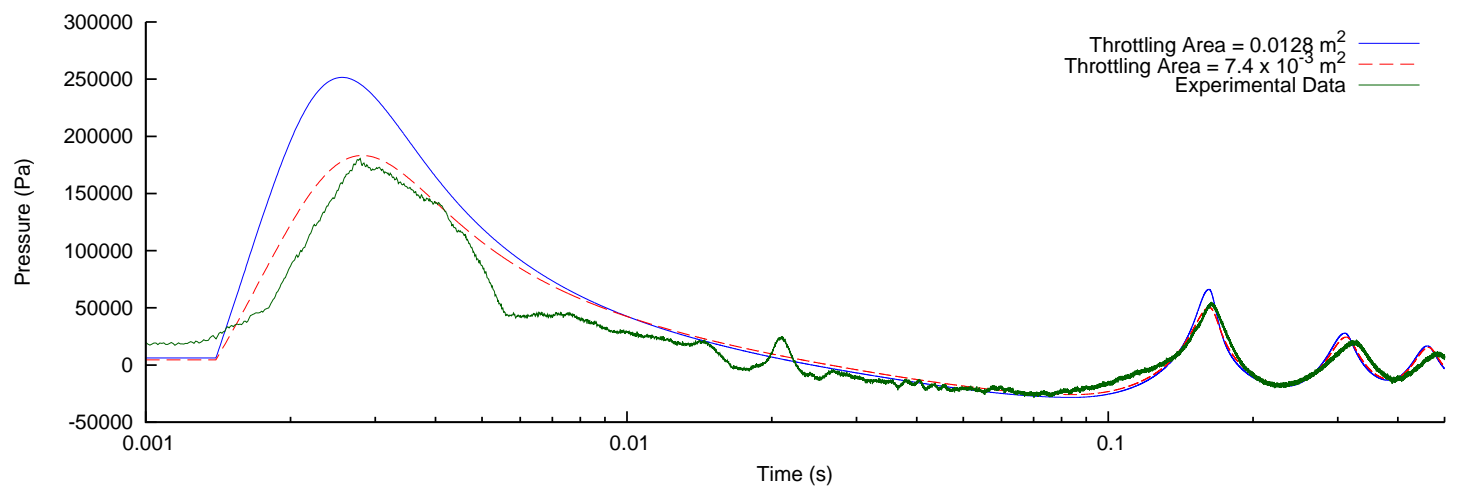

(a) Firing A

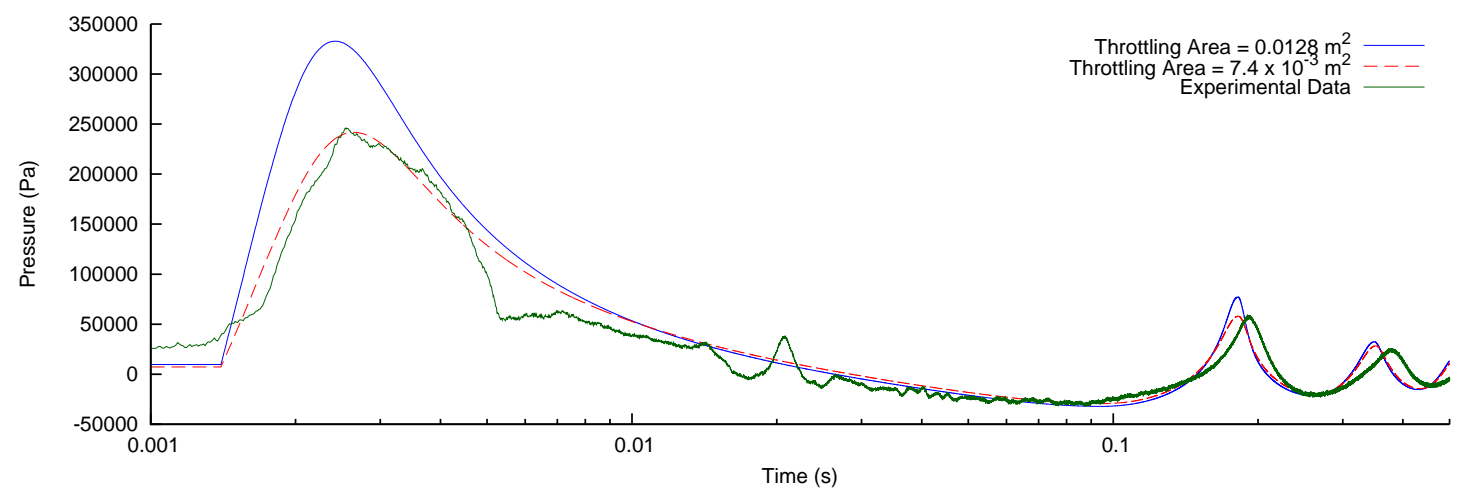

(b) Firing B

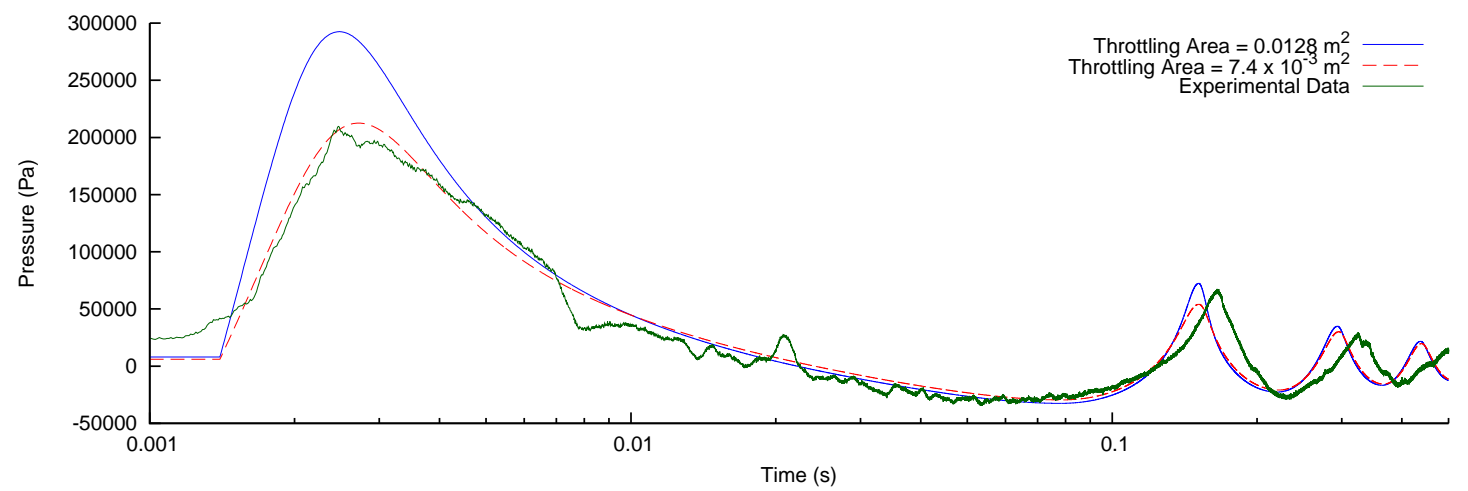

(c) Firing $\mathrm{C}$

Figure 3.8: Comparison of waveform produced by model, with (i) throttling area of 0.0128 $\mathrm{m}^{2}$ based on actual port size and (ii) predicted throttling area of $0.0074 \mathrm{~m}^{2}$, with full-scale experimental results: (a) Firing A, (b) Firing B and (c) Firing C. 


\section{Acknowledgements}

This work has been funded by the Maritime Platforms Division of the Defence Science and Technology Organisation (DSTO) and the authors wish to acknowledge the support of Dr Stuart Cannon and Mr Warren Reid. 


\title{
The Pressure Field Generated by a Seismic Airgun
}

This chapter has been submitted for publication with Experimental Thermal and Fluid Science. The contributing authors are: K.L. de Graaf, P.A. Brandner and I. Penesis.

\begin{abstract}
A model-scale seismic airgun is used to investigate the behaviour and pressure field of the bubble generated at different standoff distances from a steel plate and a free surface in an open top tank. The airgun is fired at 50 and 100 bar initial pressures and the field pressure, wall pressure and wall acceleration are recorded. Wavelet and Fast Fourier Transforms are used to analyse the bubble frequency. The reduction of pressure with distance from both the initial shock and first bubble collapse are presented. The hydrodynamic component of the pressure signal generated by the bubble collapses is also discussed. The trend of the bubble period for different standoff distances from the free surface is compared with other data in the literature and found to be similar. The acceleration and displacement of the steel plate are presented for varying bubble standoff distances, and as the plate moves in phase with the bubble, little pressure is felt from the collapse pulses. This information provides basic understanding of the dynamics of an airgun bubble when considering their application as a method of shock testing naval ships.
\end{abstract}




\subsection{Introduction}

Seismic airguns are primarily used as an impulsive pressure source in marine exploration for surveys of the ocean floor subsurface. Multiple airguns are fired simultaneously in an array to produce the single shock required for reflection and refraction surveys. Geophysicists require an understanding of the bubble dynamics and the emitted pressure signal of a single airgun in order to better understand the interactions between several airguns and the ultimate useable shock signature. Much of the research is focused on synchronising the array to produce one strong shock and minimise the following pressure pulses due to bubble oscillations.

Experimental airgun investigations are generally carried out using full-scale airguns. Exceptions to this include studies undertaken with a small commercial BOLT 600B airgun (firing volume $26 \mathrm{~cm}^{3}$ ), in which holographic and high-speed images and signature measurements were acquired $[45,46]$. Further investigations with this airgun focused on the effects of varying water temperature and viscosity on the emitted signature [43, 44]. High-speed camera images of the bubble from a $163 \mathrm{~cm}^{3}$ airgun were presented by Bungenstock [6]. Some frequency spectra of the pressure signal were also given.

Full-scale measurements have been analysed for use in developing numerical models of the generated pressure signature. These include Ziolkowski [89], who found that Gilmore's equation with a polytropic constant of 1.13 gave good results for the first period of oscillation. Johnston [36] compared the performance of airguns fired at 138 and 414 bar. Laws et al. [50] compared three methods of prediction of the pressure field generated by an airgun array through experimental testing. Vaage et al. [84] analysed measurements of airgun array firings to develop relationships between varying parameters. de Graaf et al. $[16,19]$ developed a numerical model of the growth and pressure field of an airgun bubble which compared well with full-scale airgun trial results.

Recently, there has been an interest in the use of seismic airguns for shock testing naval vessels. The Australian Government Defence Science and Technology Organisation (DSTO) has completed two trials with full-scale commercial SERCEL airguns in which field pressures and the response of a small-scale hull to varying shocks were measured $[12,71]$.

Research done on bubble behaviour near boundaries is often done in the field of cavitation in which spark and laser-generated bubbles are used to investigate the dynamics, period and radiated pressure from oscillating bubbles, for example [7, 11, 49, 54, 81] ([11] are free-field results only). Recent experiments conducted with underwater explosions focusing on bubble dynamics near a boundary include Klaseboer et al. [42] and Hung and Hwangfu [35]. 
This paper presents the results from a series of experiments conducted at the Australian Maritime College (AMC) with a purpose-built laboratory scale airgun, modelled on the airguns used by DSTO. These experiments study the field and wall pressures produced by the airgun when fired at two different pressures at varying standoff distances from a steel wall and depths from a free surface in an open top tank.

\subsection{Experimental Setup}

\subsubsection{Laboratory Scale Airgun}

A laboratory scale airgun has been designed based on a typical four port airgun. The airgun has a firing volume of $14.5 \mathrm{~cm}^{3}$ and can be pressurized up to 100 bar from a standard dive bottle. Major external dimensions of the airgun are given in Figure 4.1. The cylindrical airgun body has four $20 \mathrm{~mm}$ wide by $8 \mathrm{~mm}$ high ports through which the air is released, and is fitted to a pipe of equal outside diameter, as shown in Figure 4.2 .

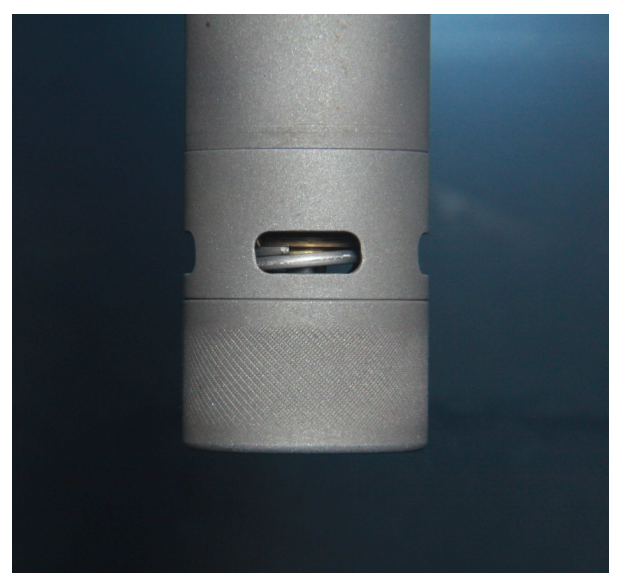

(a) Image of model-scale airgun

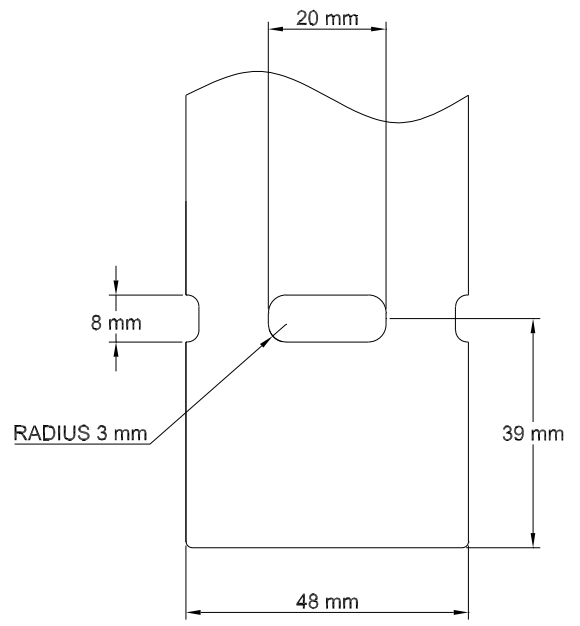

(b) Major external dimensions of the model-scale airgun

Figure 4.1: Model-scale airgun.

The basic operation of an airgun is similar across the commercial range and uses a pressure differential to fire a shuttle which rapidly releases a fixed volume of compressed air to form a bubble. Due to the small size of this airgun and the limits in valve sizes and drilling diameters, a central control rod creates the air passages required to facilitate charging and firing the airgun. 


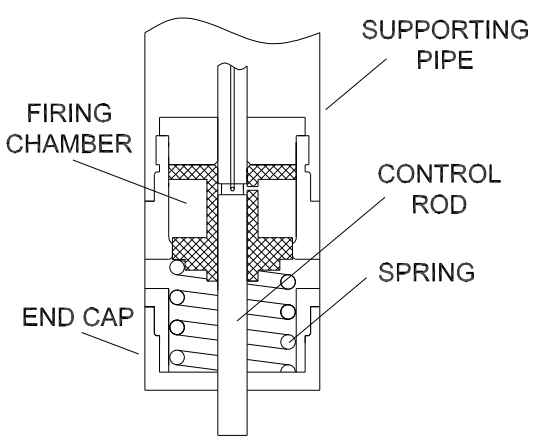

(a)

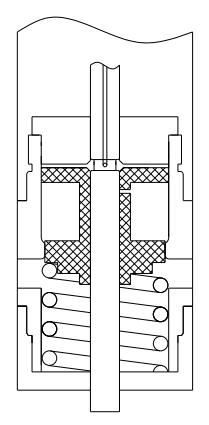

(b)

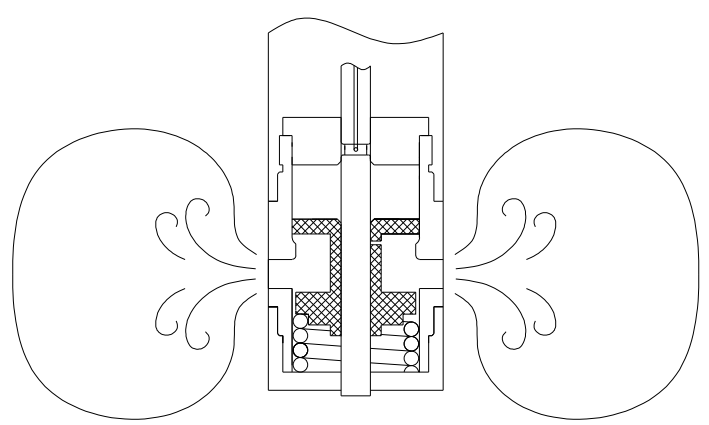

(c)

Figure 4.2: Illustration of the model airgun firing in three stages: (a) pressurised chamber under equilibrium; (b) equalisation of pressure across upper flange to fire shuttle and (c) release of air through ports to form bubble.

Initially, the control rod is positioned to allow the firing chamber to be charged with compressed air (Figure 4.2a). The shuttle is held in place by the pressure difference between the two ends of the shuttle as the upper flange of the shuttle is slightly larger than the lower flange. The control rod is then moved up, exposing the back of the upper flange to the firing pressure (Figure 4.2b) and resulting in a net force acting on the lower flange such that the shuttle opens, discharging the compressed air through the four ports (Figure 4.2c). The control rod moves back into the body to prevent excess additional air holding the shuttle open. A spring returns the shuttle to its closed position and the control rod is then reset.

\subsubsection{Testing Tank and Equipment}

Experiments were carried out in the AMC Cavitation Research Laboratory. The airgun was suspended vertically in the centre of a $1.728 \mathrm{~m}^{3}$ open top water tank with dimensions of $1.2 \mathrm{~m} \times 1.2 \mathrm{~m} \times 1.2 \mathrm{~m}$. The tank is constructed with one $16 \mathrm{~mm}$ stainless steel plate side and the remaining three sides and base of $50 \mathrm{~mm}$ clear acrylic. A Brüel \& Kjær (B\&K) Type 8103 hydrophone and B\&K 2692 signal conditioning amplifier were used to measure the bubble pressure pulse in the field at varying distances from the airgun. A B\&K Type 4507 B004 Deltatron accelerometer and PCB 112A21 pressure transducer, both of which were used with a PCB Piezotronics 482C signal conditioner, were located on the steel wall to measure the wall pressure and acceleration. The pressure transducer was located at the centre of the plate with the accelerometer adjacent. The airgun was fired using a solenoid (GTUW 070 T43 A01) to move the control rod upwards. A trigger pulse on firing was directed via a variable delay (Thurlby Thandar Instruments TGP110) 


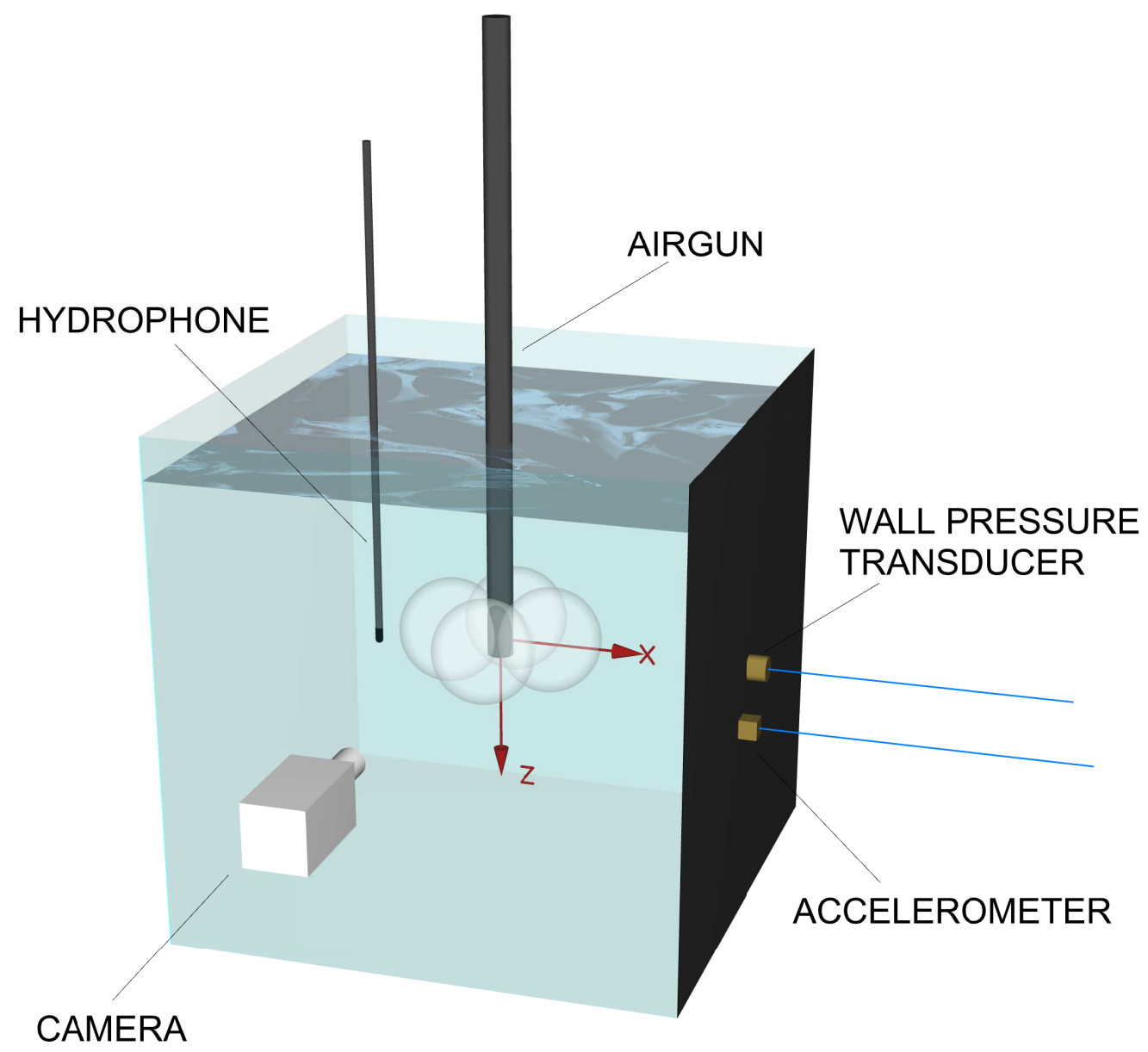

Figure 4.3: Experimental Setup. The $1.2 \mathrm{~m} \times 1.2 \mathrm{~m} \times 1.2 \mathrm{~m}$ tank has one $16 \mathrm{~mm}$ stainless steel plate side, and three sides and a base of $50 \mathrm{~mm}$ clear acrylic. The airgun and a hydrophone are suspended vertically from a rail spanning the tank which permits their positions to be independently adjusted vertically and horizontally. The wall pressure transducer is located at the centre of the stainless steel plate with the accelerometer adjacent. 
and used to initialise data recording from the pressure transducers and accelerometer. Data from the two pressure transducers and the accelerometer were recorded at $100 \mathrm{kHz}$ using a National Instruments (NI) PCI-4472 simultaneous sample and hold acquisition card. In addition to the dynamic data, the charged airgun pressure and ambient pressure were measured and recorded using a Wika S-10 pressure transmitter on the air supply and a Vaisala PTB 210C4C2M barometer, respectively, via a NI PCI-6289 acquisition card. Bubble radii were derived from high-speed photography recordings acquired at 3 $\mathrm{kHz}$ using a La Vision High-Speed Star 5 camera. A diagram of the experimental setup is shown in Figure 4.3 and a schematic of the data acquisition equipment in Figure 4.4.

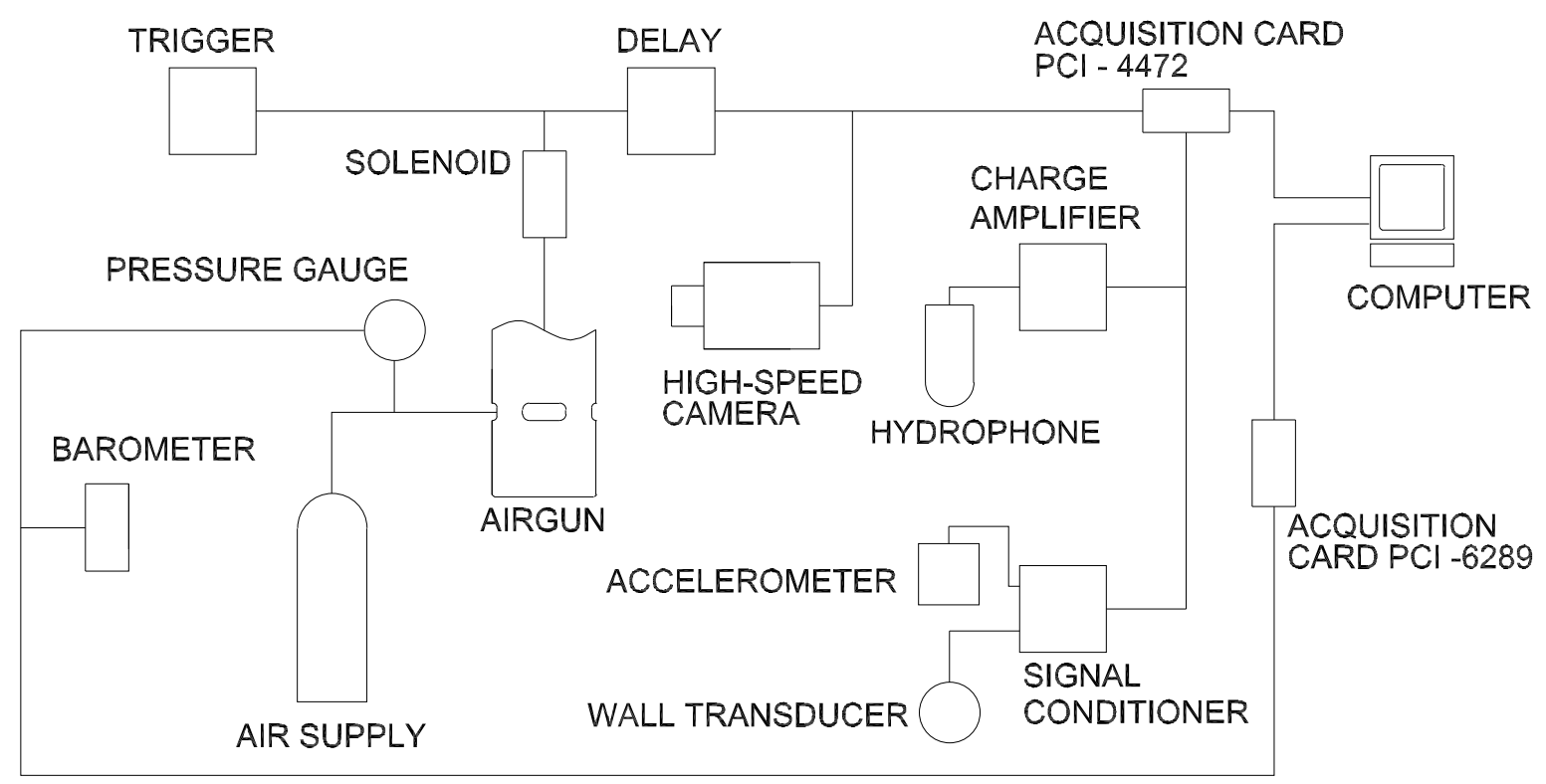

Figure 4.4: Schematic showing the data acquisition equipment setup.

The first set of experiments focused on determining the pressure response at varying distances from the airgun. Results were obtained for 50 and 100 bar initial airgun pressures for the cases where the hydrophone was traversed away from the airgun (at the same depth as the bubble) and where the airgun was progressively moved toward the steel plate (with the hydrophone position fixed). The vertical changes in the pressure field were tested by lowering and raising the hydrophone at one horizontal offset with respect to the fixed depth airgun. To assess the directivity of the airgun due to the arrangement of the four ports (symmetric about four axes), the airgun was rotated in 15 degree increments through a total of 45 degrees. Interaction of the bubble with the free surface was studied by reducing the water level. The airgun and hydrophone remained in the same position relative to the tank to enable photography and pressure transducer results to be obtained at the same depth as the bubble. 
An image taken at the time the bubble reaches its first maximum radius is shown in Figure 4.5. This figure shows the four distinct bubbles developed from the jets of air released through the four ports of the airgun. The dynamics of the bubble are discussed in detail in [18] (Chapter 5).

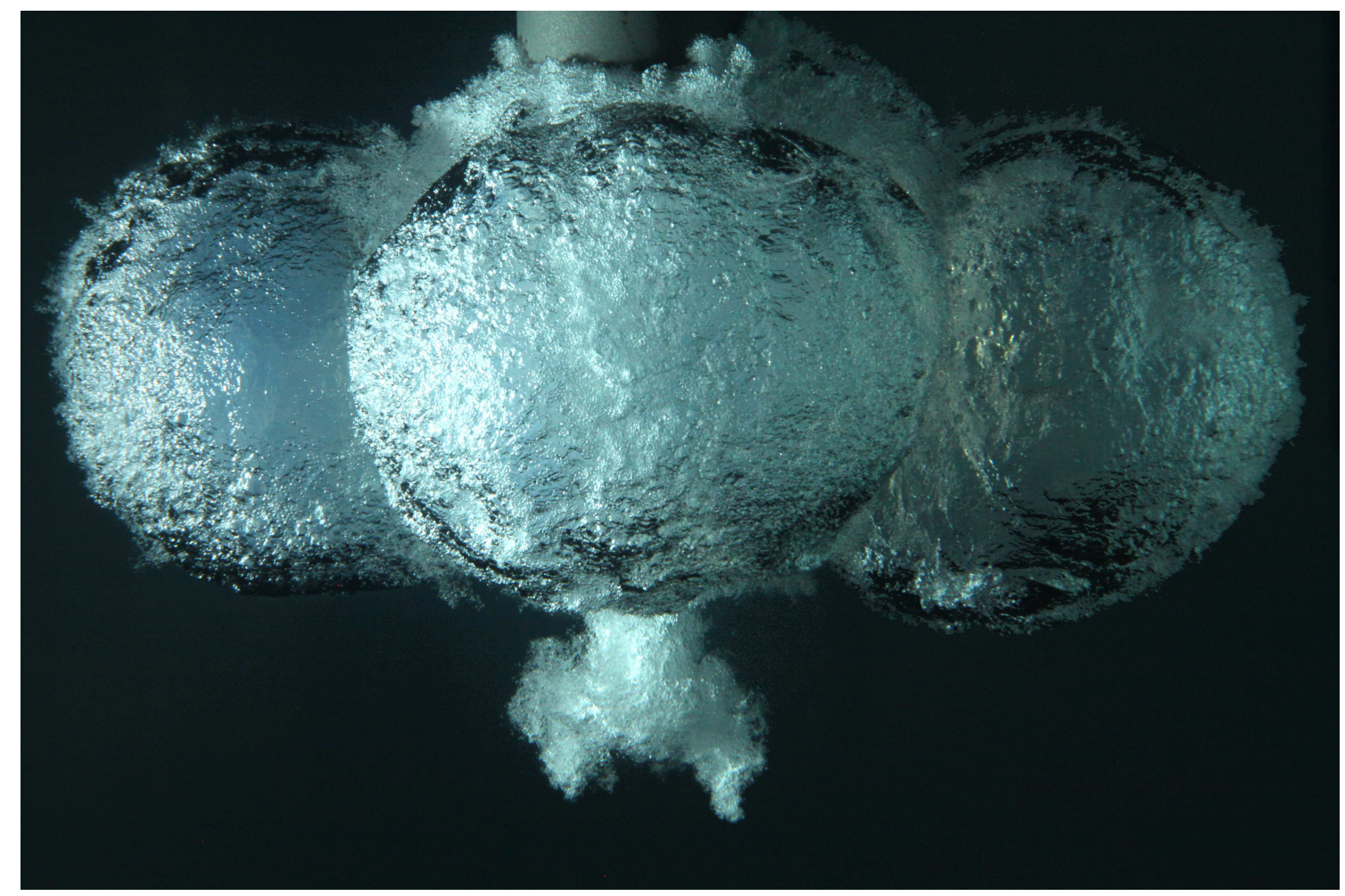

Figure 4.5: The bubble generated by the model-scale seismic airgun. The bubble is just reaching its first maximum radius and four distinct bubbles are evident. These are the result of the release of air through the four airgun ports.

\subsection{Results and Discussion}

\subsubsection{Pressure Signal}

Typical field pressure responses for initial airgun pressures of 50 and 100 bar are shown in Figure 4.6. The hydrophone was located $191 \mathrm{~mm}$ away from the airgun. The bubble pulsing and tank reverberant frequencies from the initial shock are evident in the signals. The bubble frequencies are approximately 70 and $50 \mathrm{~Hz}$ for the 50 and 100 bar initial airgun pressures respectively, compared with about $1000 \mathrm{~Hz}$ for the reverberant frequency for each case. When the airgun is fired, an initial shock is emitted (point 1 in Figure 4.6) followed closely by a second shock (point 2). This subsequent pressure pulse is not typically seen in airgun waveforms, but is due to particular jetting dynamics evident 
from the high-speed photography. The dynamics of the bubble are discussed in detail with accompanying images in [18] (Chapter 5). Points 3 and 4 show the pressure pulses produced by the first and second bubble collapses. As the bubble continues to pulse, the strength of the pressure pulses decays with time through viscous losses from turbulence, heat transfer and radiated acoustic energy [19] (Chapter 3).

Pressure and time are non-dimensionalised using the difference between the initial airgun pressure, $p_{g}$, and ambient (atmospheric plus hydrostatic) pressure, $p_{\infty}$, and Minneart's frequency, $f_{0}$, for an equivalent equilibrium bubble, respectively. Minneart's equation for natural bubble frequency is given by:

$$
f_{0}=\frac{1}{2 \pi r_{0}} \sqrt{\frac{3 k p_{\infty}}{\rho}}
$$

where $f_{0}$ is the natural bubble frequency, $r_{0}$ is the equivalent equilibrium bubble radius at ambient pressure, $k$ is the polytropic coefficient, taken as the adiabatic index (1.4) and $\rho$ is the water density. An equivalent equilibrium bubble is one with the same air mass as contained in the pressurised airgun firing chamber, if expanded isothermally to ambient pressure.

\subsubsection{Repeatability}

To determine the pressure variation with distance, five firings were recorded at each location for both initial airgun pressures. The repeatability of the airgun is shown using the field pressure response data in Figures 4.7 (raw data) and 4.8 (filtered data). The initial shock is very transient and as a result there is more variation in its magnitude compared with the magnitude of the pulses generated by the following bubble collapses. Where appropriate, 'error bars' have been used to represent the range of measured data (maximum and minimum measured values) obtained in the corresponding multiple firings. Accordingly, the ranges given for initial shock measurements are larger than those for pressure pulses generated by the bubble collapses.

\subsubsection{Bubble Pulse and Reverberation}

The raw data from typical field pressure responses has been filtered to extract the bubble pulse and the decay of the reverberation in the tank. A low and high pass finite impulse response filter with a cut-off frequency of $900 \mathrm{~Hz}$ was used to extract the two frequencies. No filtering window was used as the signal is transient and fully captured within the acquisition period. The low pass filter was not implemented until the time at which 

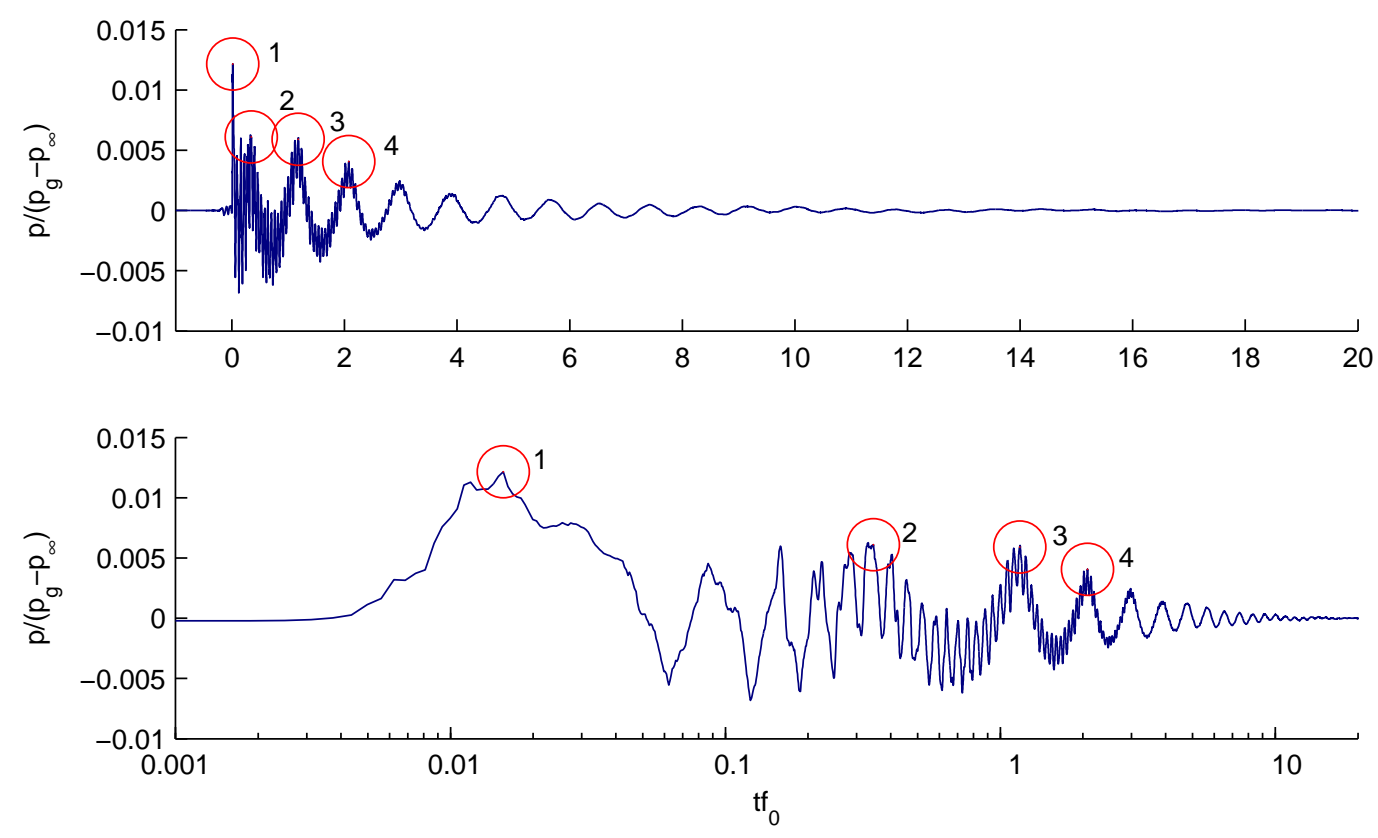

(a) 50 bar initial pressure
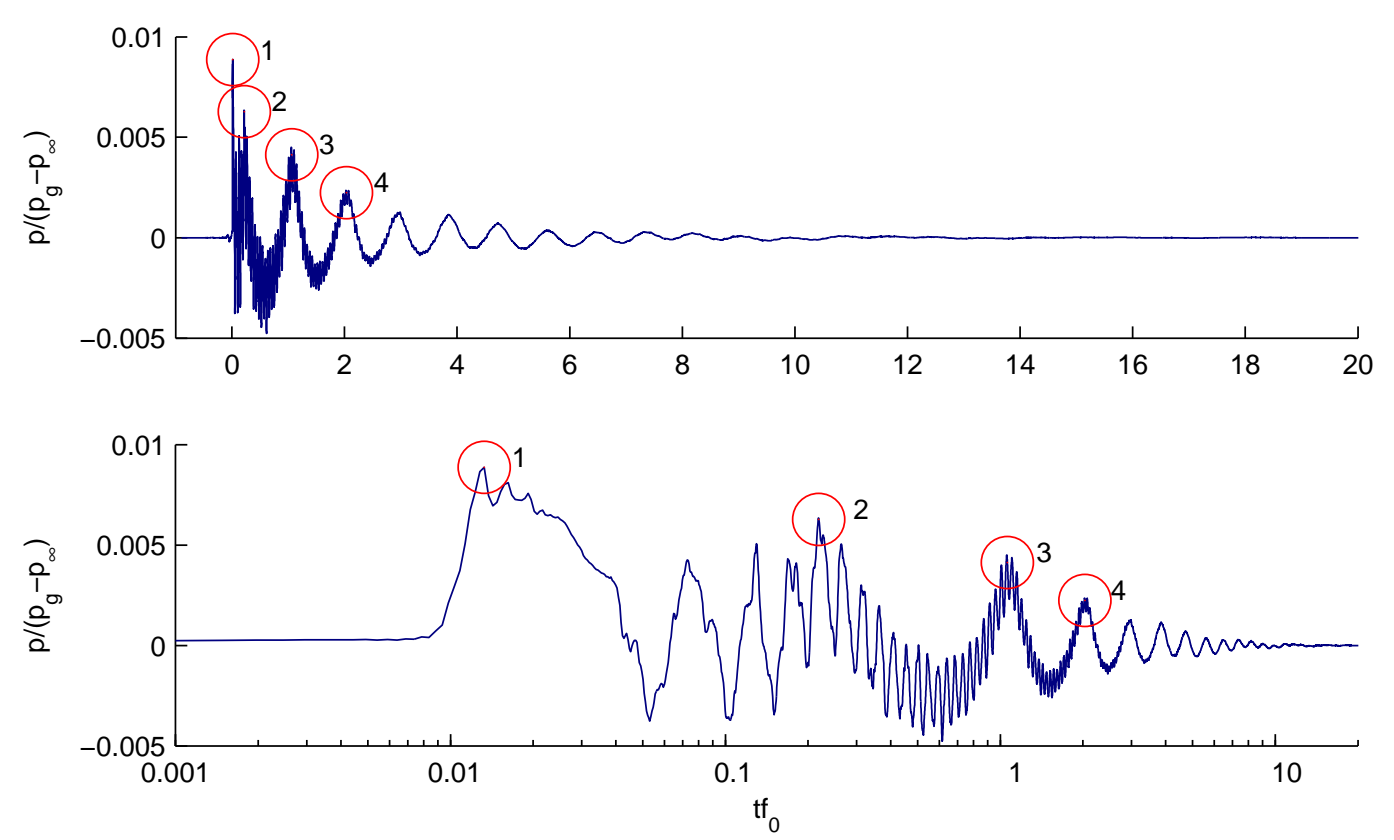

(b) 100 bar initial pressure

Figure 4.6: Typical field response at a standoff distance of $191 \mathrm{~mm}$ for initial airgun pressures of 50 and 100 bar (standard scale and $\log$ scale shown for clarity). Point 1: initial shock at firing. Point 2: additional pressure pulse caused by specific bubble jetting dynamics. Point 3: pressure pulse generated by first bubble collapse. Point 4: pressure pulse generated by second bubble collapse. Pressure and time are non-dimensionalised using the difference between initial airgun pressure, $p_{g}$, and ambient pressure, $p_{\infty}$, and Minneart's frequency, $f_{0}$, for an equivalent bubble at ambient pressure, respectively. 


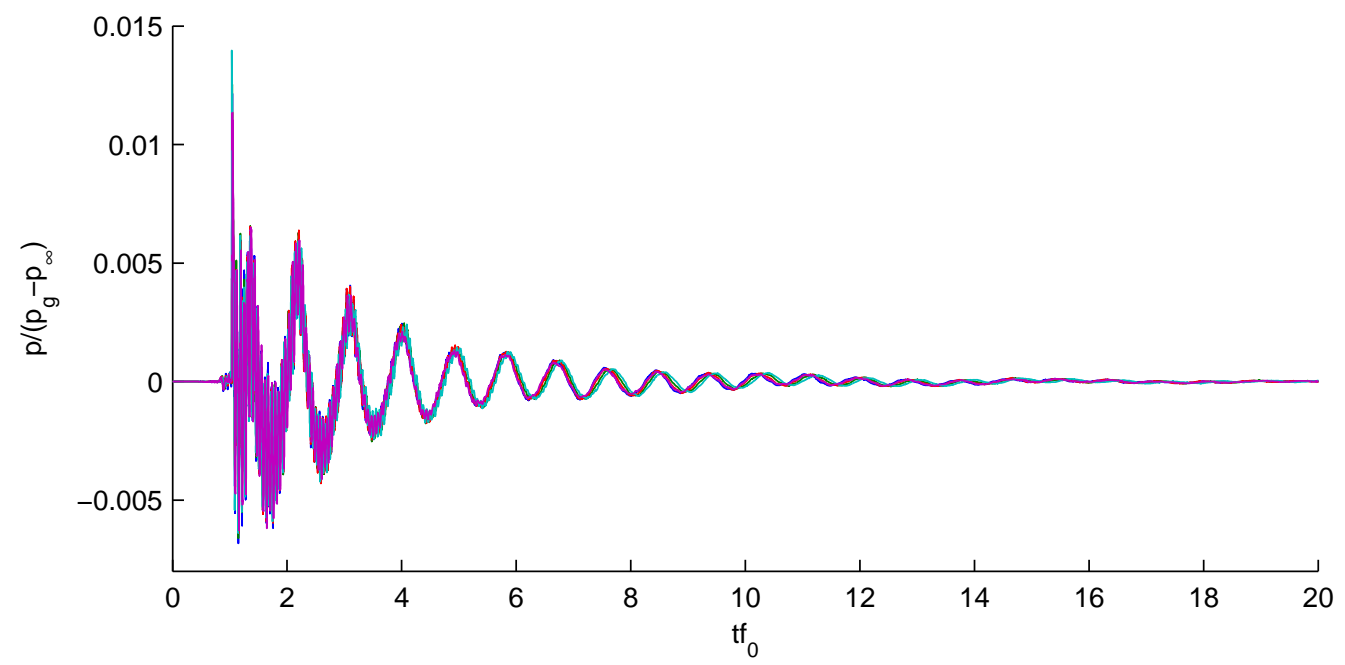

(a) 50 bar initial pressure

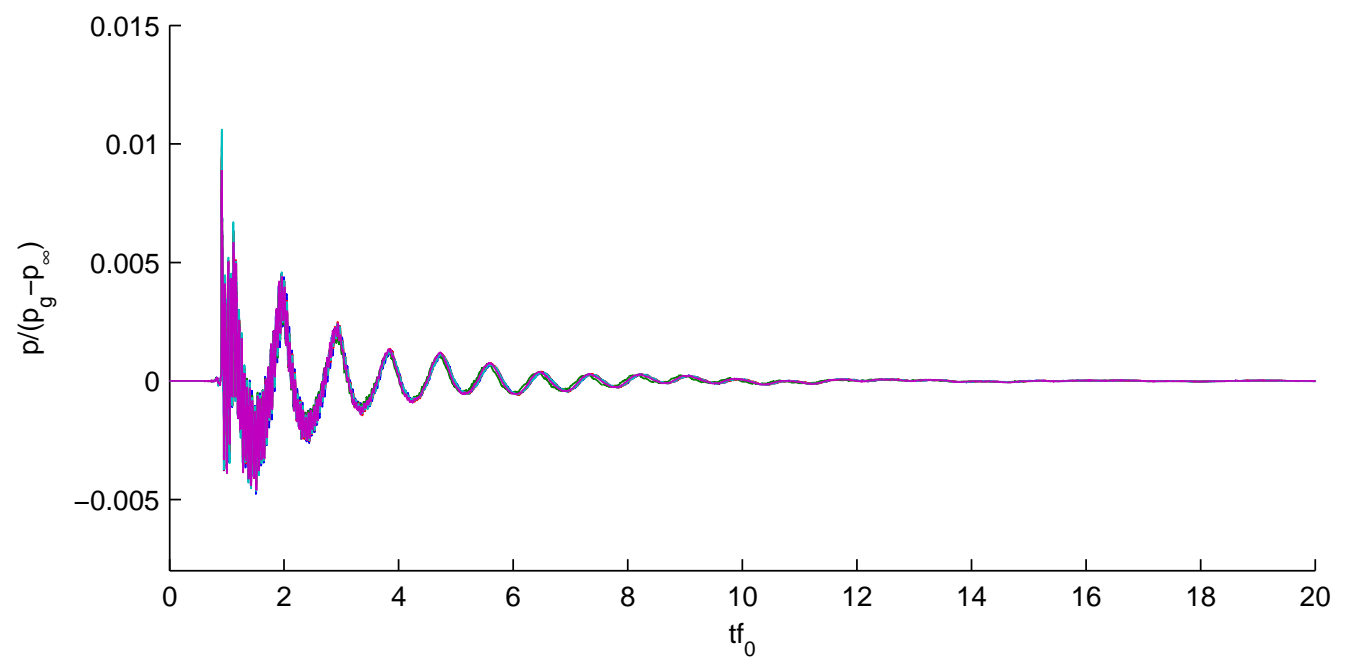

(b) 100 bar initial pressure

Figure 4.7: Repeatability of the airgun firings shown through the comparison of five raw field pressure responses. The initial shock is transient and shows more variation in magnitude compared with the pressure pulses generated by the bubble collapses. Signals have been aligned in time at the point of initial shock. Pressure and time are non-dimensionalised using the difference between initial airgun pressure, $p_{g}$, and ambient pressure, $p_{\infty}$, and Minneart's frequency, $f_{0}$, for an equivalent bubble at ambient pressure, respectively. 


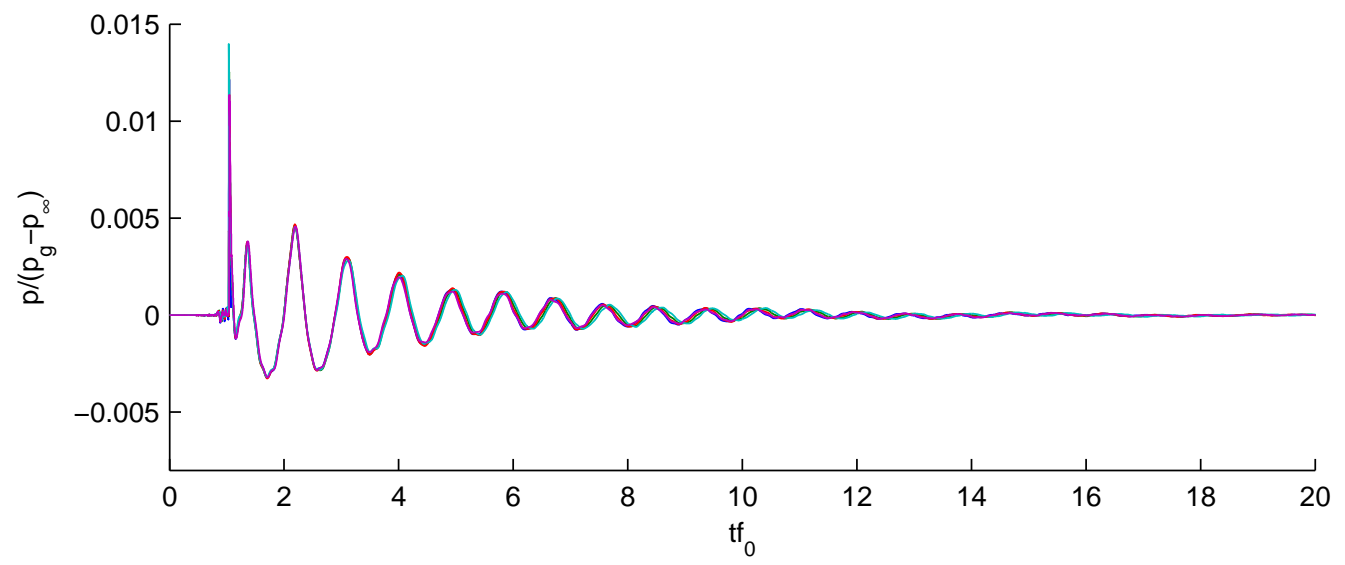

(a) 50 bar initial pressure

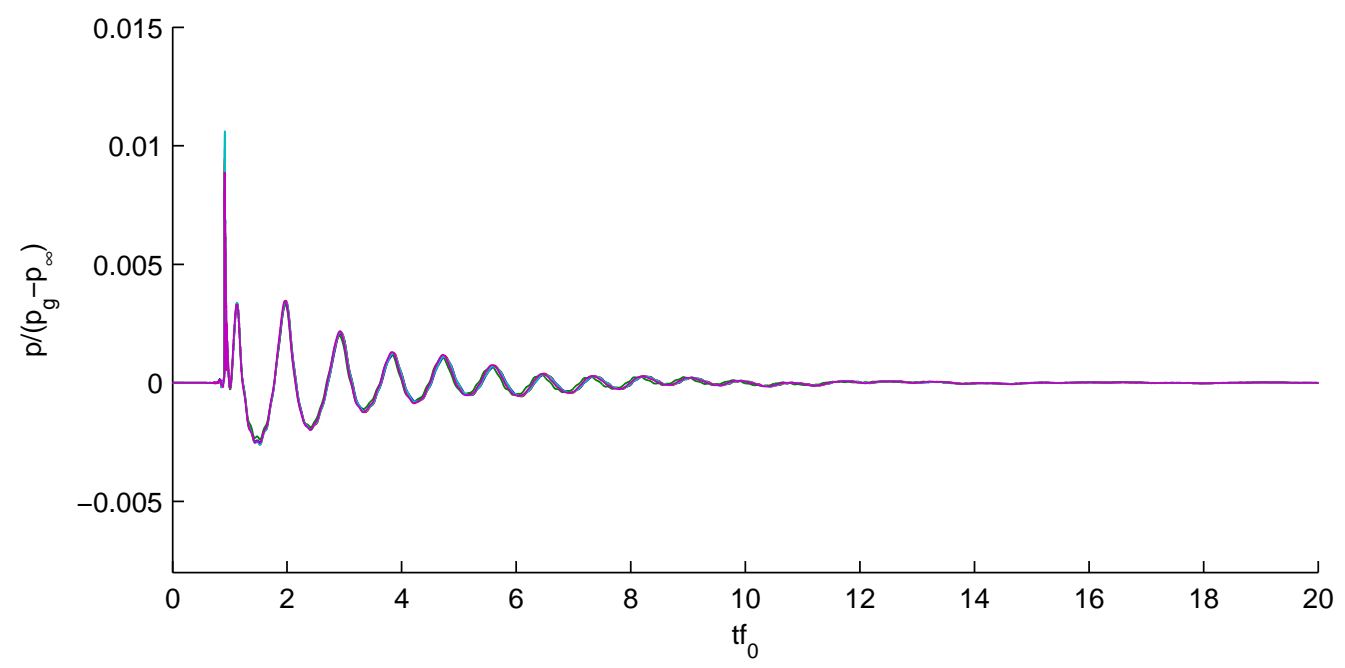

(b) 100 bar initial pressure

Figure 4.8: Repeatability of the airgun firings shown through the comparison of five filtered field pressure responses. See Section 4.3.3 for details of the filtering method. The initial shock is transient and shows more variation in magnitude compared with the pressure pulses generated by the bubble collapses. Signals have been aligned in time at the point of initial shock. Pressure and time are non-dimensionalised using the difference between initial airgun pressure, $p_{g}$, and ambient pressure, $p_{\infty}$, and Minneart's frequency, $f_{0}$, for an equivalent bubble at ambient pressure, respectively. 
the reflection of the initial shock reaches the sensor, preserving the transient shock, rather than smoothing it out with the filter. For each result, a least squares fit was used to find the four variables of the damped harmonic equation. Results for the airgun fired at 50 and 100 bar are shown in Figures 4.9 and 4.10, respectively. The equations given are for the cases before the data is non-dimensionalised, in order to show the actual frequency and decay values. The frequencies of the bubble and the reverberation found by fitting the damped equation compare well with those found from the wavelet and FFT methods. The exponential decay coefficient of the oscillating bubble pulses is -22.10 and -22.02 for the 50 and 100 bar initial pressure cases, respectively. The decay of the reverberation is slightly faster for the 50 bar case due to the weaker initial shock magnitude. The separation of the two signals makes evident the faster reverberation decay of the initial shock compared with the hydrodynamic pressure from the bubble pulsation. The frequency of both the bubble pulse and the reverberation change slowly with time, which is unable to be replicated by the damped equation. The asymmetry of the peaks and troughs in the bubble pulse also limits the accuracy when modelling with a sinusoidal wave.

\subsubsection{Wavelet Transform and FFT Analysis}

The continuous wavelet transform method of Torrence and Compo [82] is used in addition to Fast Fourier Transforms (FFTs) to analyse the recorded pressure and acceleration responses. Wavelet transforms better identify local frequencies and individual events within a transient signal, as opposed to the dominant global frequencies identified by an FFT. A Morlet wavelet with wavenumber equal to 6 is used in the analysis as it is a common complex wavelet and good for oscillatory behaviour [82] and separating multiple vibration modes [1]. As the signal is transient, the FFT is calculated with no windowing over the full $10^{5}$ samples. Frequency has been non-dimensionalised using Minneart's frequency, $f_{0}$, for an equivalent bubble, as described above.

The wavelet transform of typical signals received by the hydrophone in the field and the pressure transducer and accelerometer on the tank wall, for 50 and 100 bar initial airgun pressures are shown in Figures 4.11 and 4.12. The airgun was located in the centre of the tank, $600 \mathrm{~mm}$ from the steel plate and wall mounted transducer, $191 \mathrm{~mm}$ from the hydrophone and at a depth of $507 \mathrm{~mm}$. The Power Spectral Densities (PSDs) from the wavelet and FFT analyses of the field pressure response for 50 and 100 bar initial pressures are compared in Figure 4.13. Both PSDs show similar overall frequency strengths and identify the dominant bubble and reverberant frequencies. 


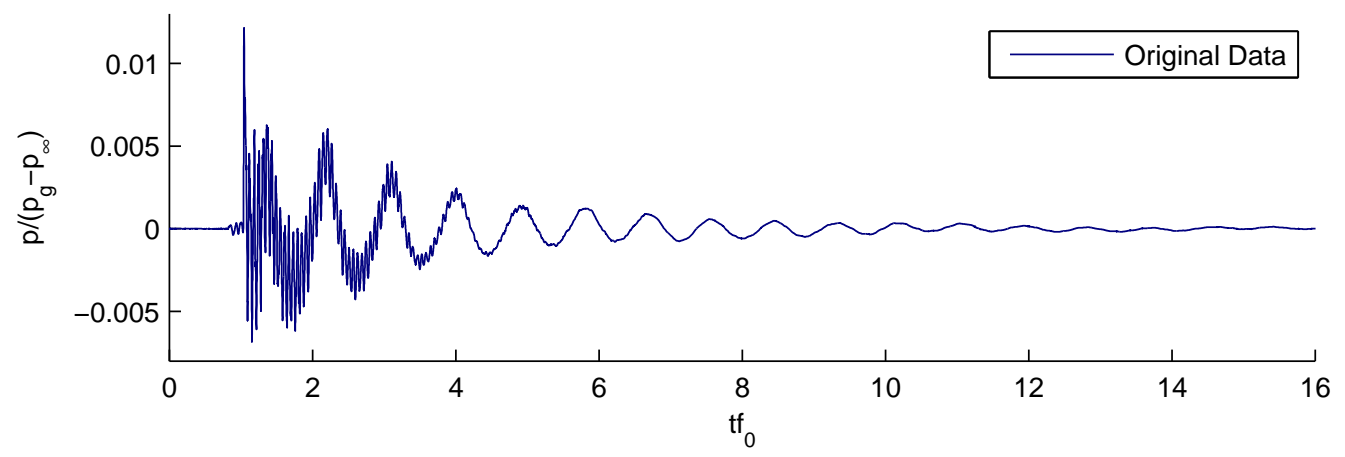

(a) Field pressure

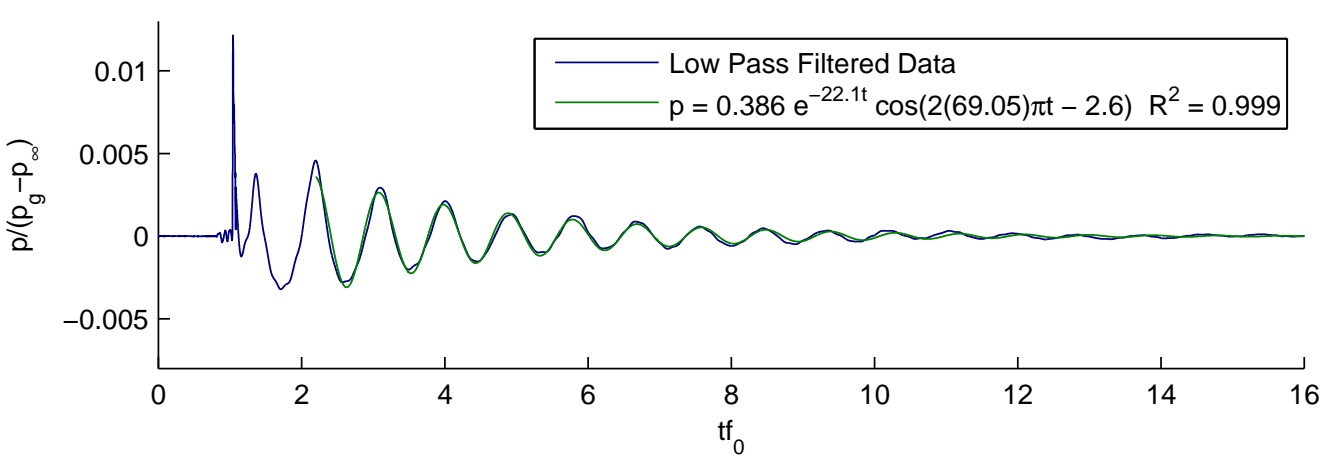

(b) Bubble pulse

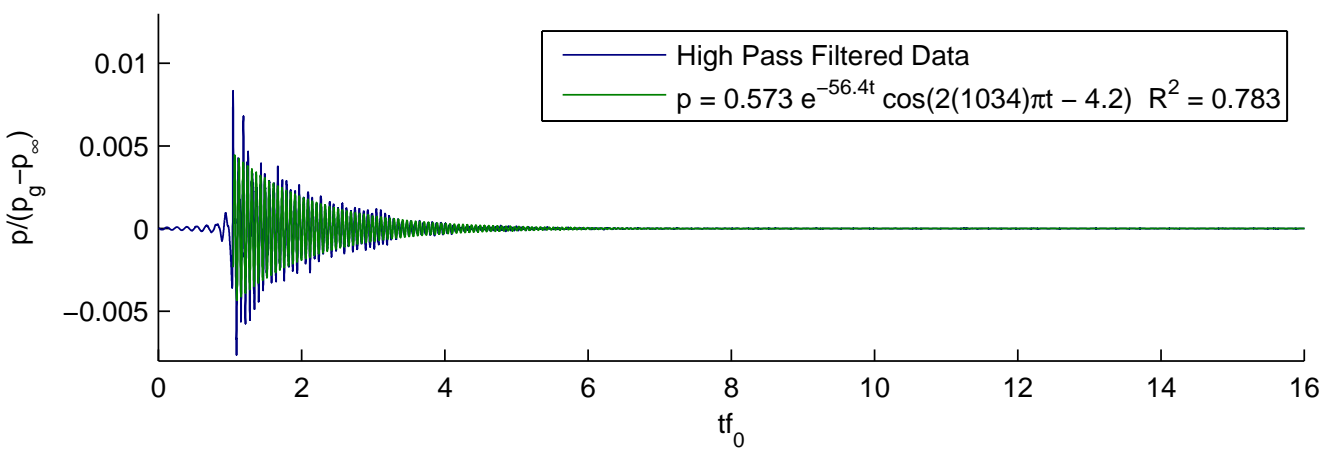

(c) Reverberation

Figure 4.9: Field pressure response for airgun fired at 50 bar initial pressure, with bubble pulse and reverberation extracted using a low and high pass finite impulse response filter, respectively. The cut-off frequency for each filter was $900 \mathrm{~Hz}$. The damped harmonic equation is fitted to the filtered data. Pressure and time are non-dimensionalised using the difference between initial airgun pressure, $p_{g}$, and ambient pressure, $p_{\infty}$, and Minneart's frequency, $f_{0}$, for an equivalent bubble at ambient pressure, respectively. 


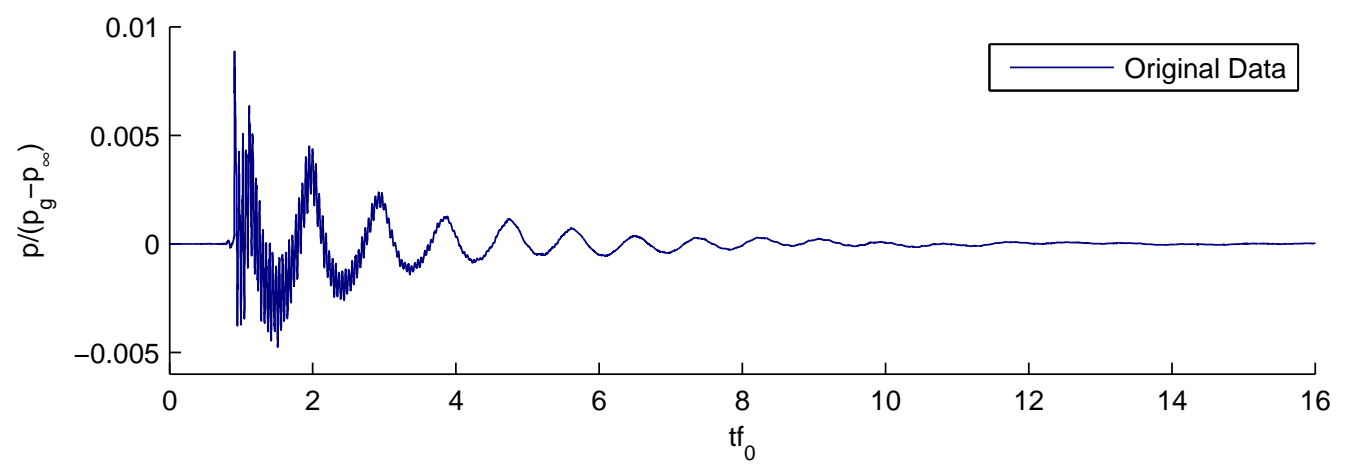

(a) Field pressure

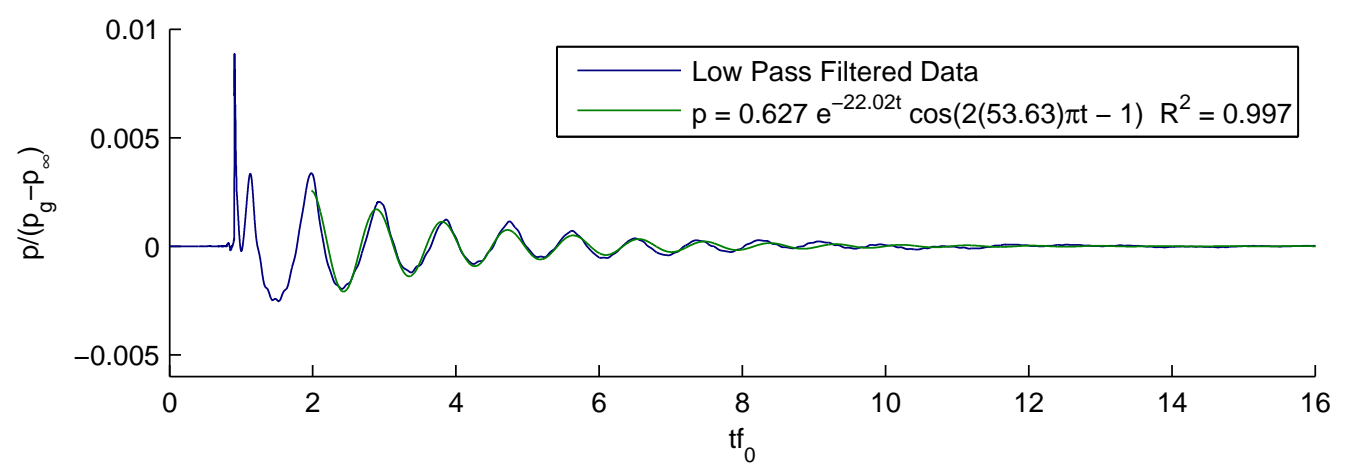

(b) Bubble pulse

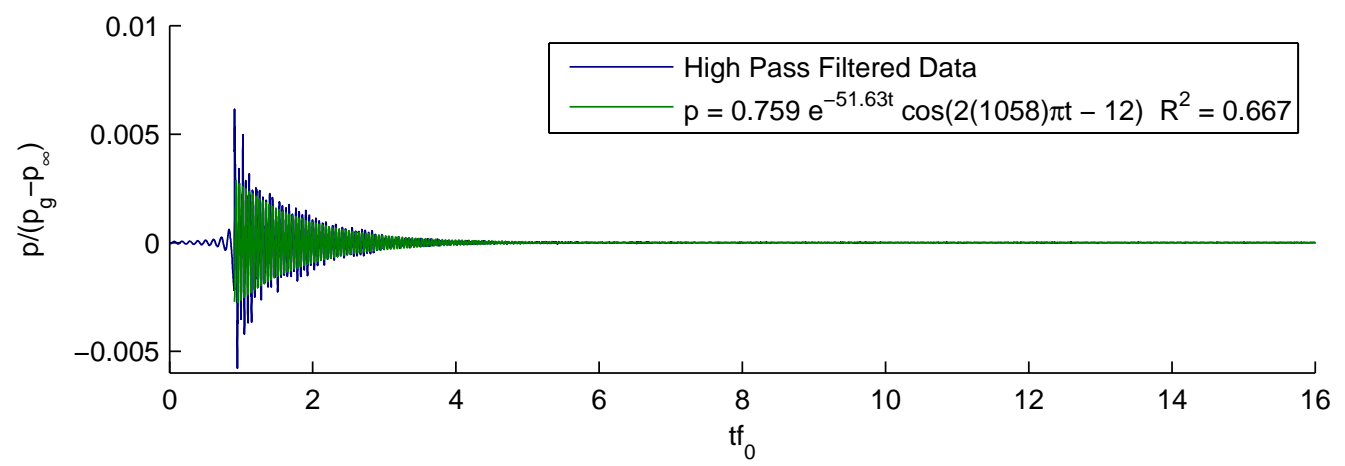

(c) Reverberation

Figure 4.10: Field pressure response for airgun fired at 100 bar initial pressure, with bubble pulse and reverberation extracted using a low and high pass finite impulse response filter, respectively. The cut-off frequency for each filter was $900 \mathrm{~Hz}$. The damped harmonic equation is fitted to the filtered data. Pressure and time are non-dimensionalised using the difference between initial airgun pressure, $p_{g}$, and ambient pressure, $p_{\infty}$, and Minneart's frequency, $f_{0}$, for an equivalent bubble at ambient pressure, respectively. 


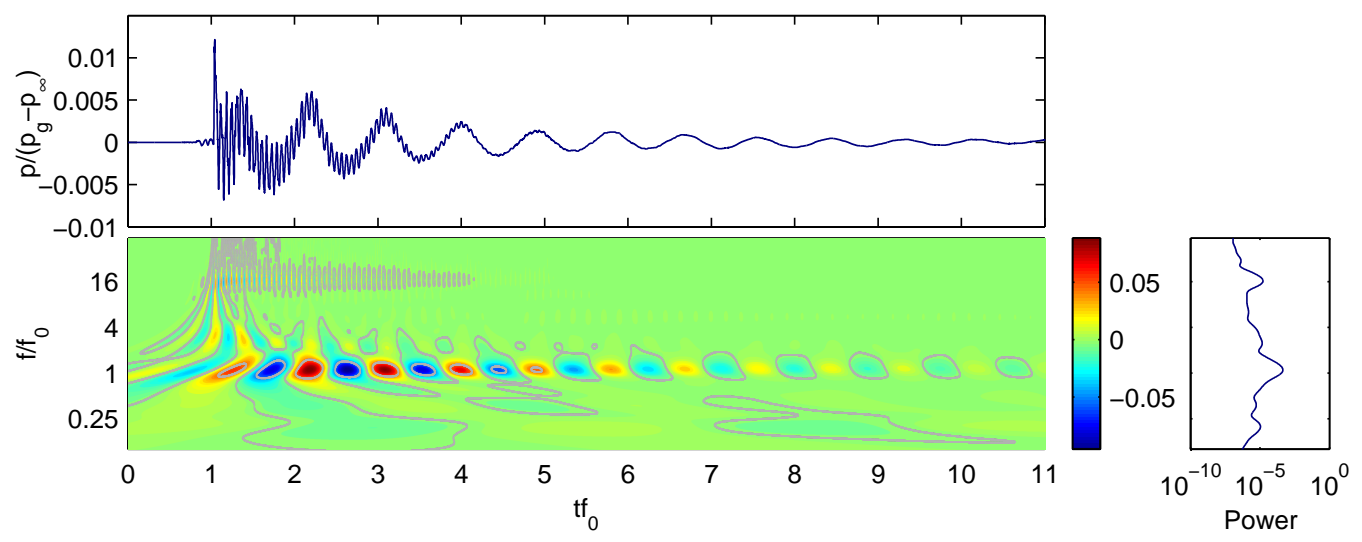

(a) Field pressure

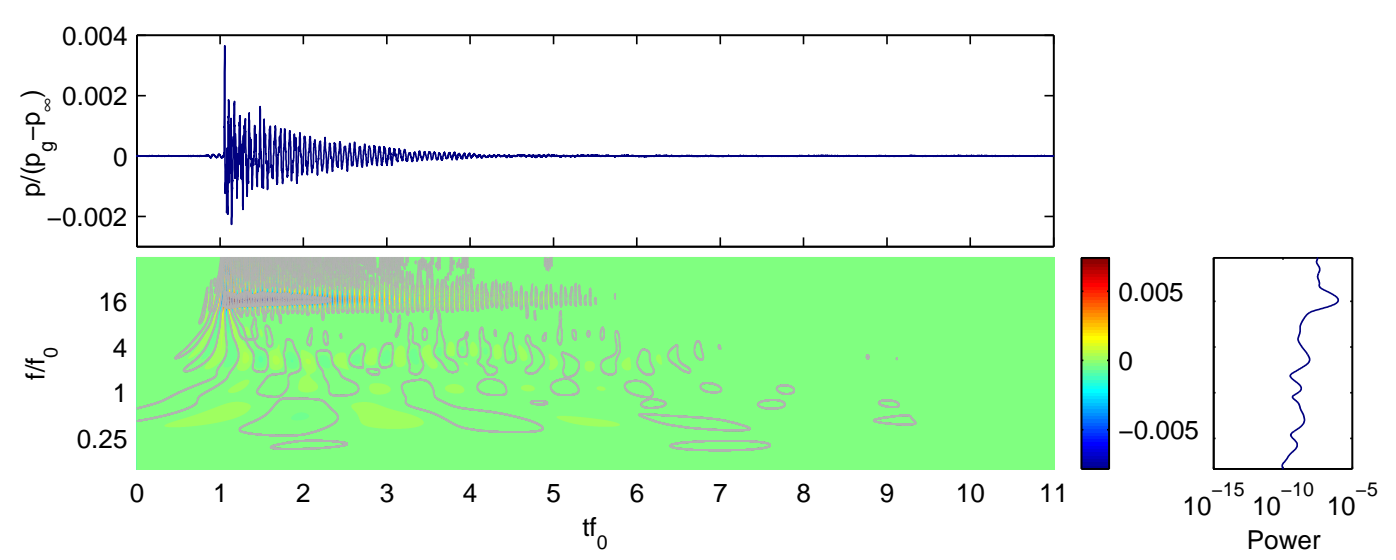

(b) Wall pressure

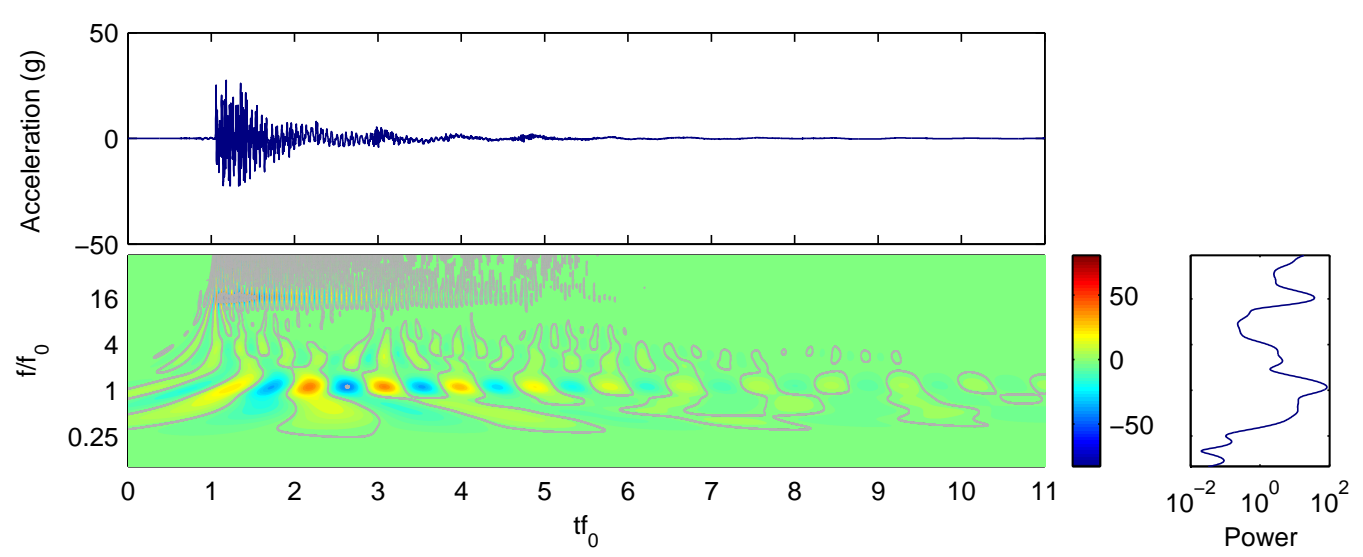

(c) Acceleration

Figure 4.11: Wavelet transforms for field pressure, wall pressure and acceleration for 50 bar initial airgun pressure. The reverberation and bubble frequencies (or acoustic and hydrodynamic components) are clearly separated in the field pressure and accelerometer responses. The wall pressure response shows the initial shock but no subsequent bubble pulses due to the phasing of the wall movement with the bubble, as evident from the bubble frequency observed in the accelerometer response. Pressure and time are non-dimensionalised using the difference between initial airgun pressure, $p_{g}$, and ambient pressure, $p_{\infty}$, and Minneart's frequency, $f_{0}$, for an equivalent bubble at ambient pressure, respectively. 


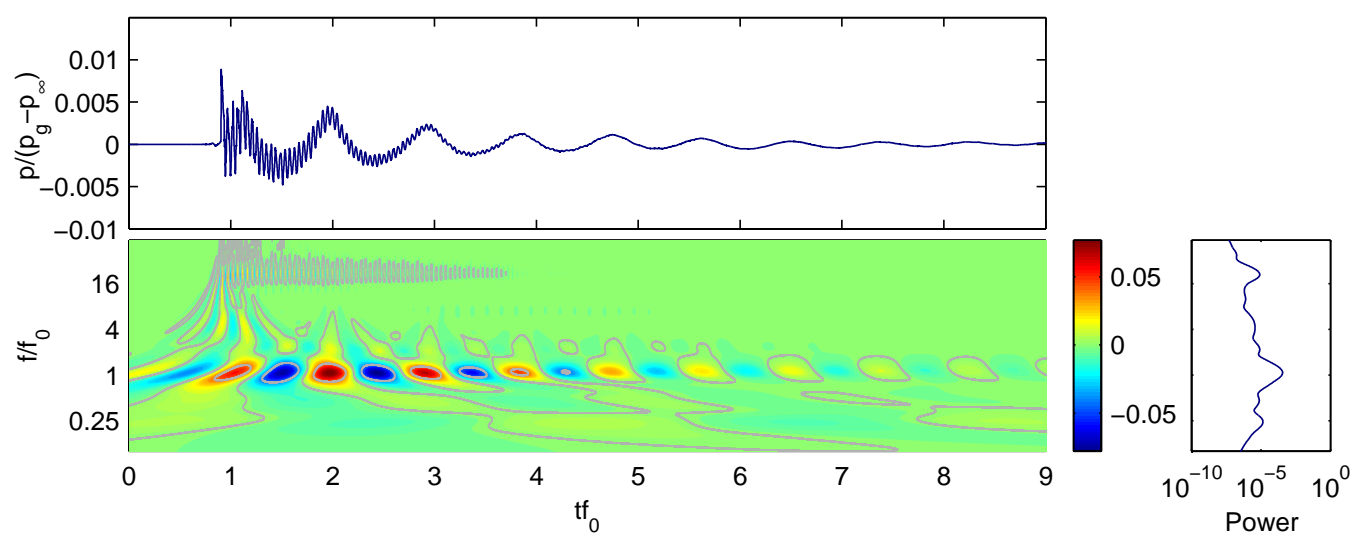

(a) Field pressure

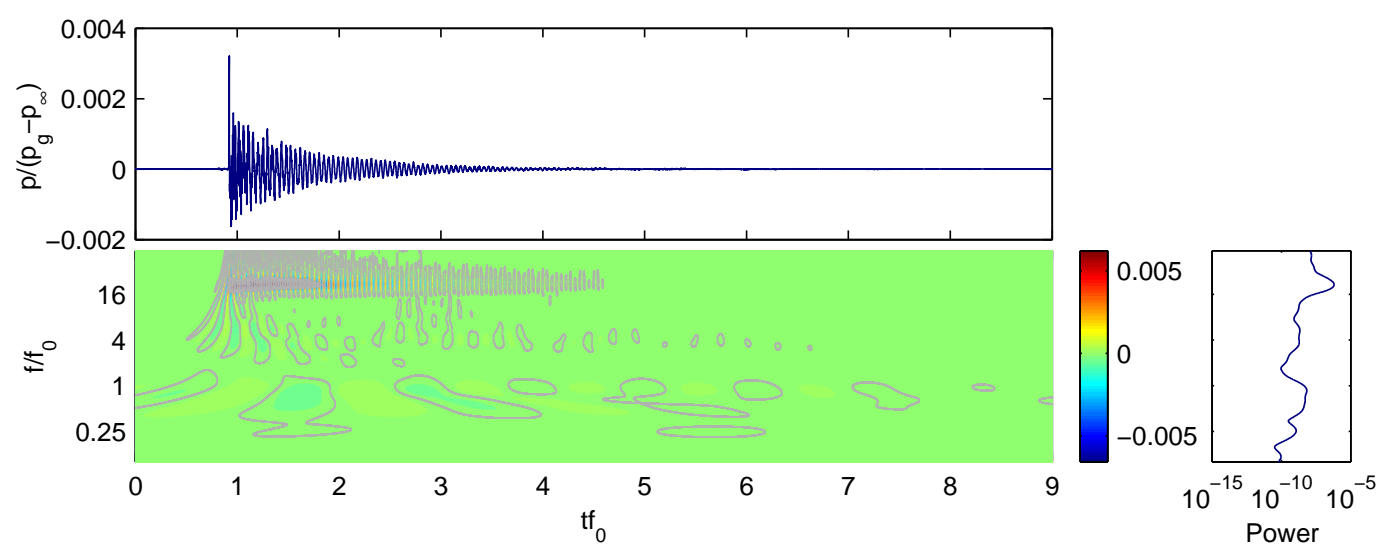

(b) Wall pressure

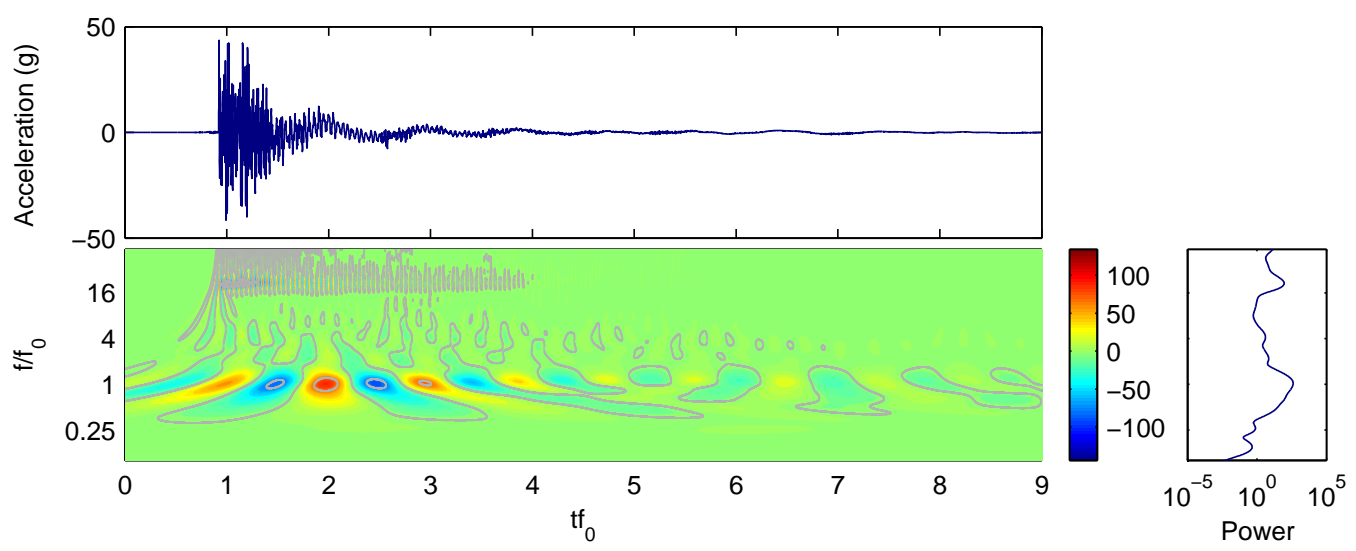

(c) Acceleration

Figure 4.12: Wavelet transforms for field pressure, wall pressure and acceleration for 100 bar initial airgun pressure. The reverberation and bubble frequencies (or acoustic and hydrodynamic components) are clearly separated in the field pressure and accelerometer responses. The wall pressure response shows the initial shock but no subsequent bubble pulses due to the phasing of the wall movement with the bubble, as evident from the bubble frequency observed in the accelerometer response. Pressure and time are non-dimensionalised using the difference between initial airgun pressure, $p_{g}$, and ambient pressure, $p_{\infty}$, and Minneart's frequency, $f_{0}$, for an equivalent bubble at ambient pressure, respectively. 


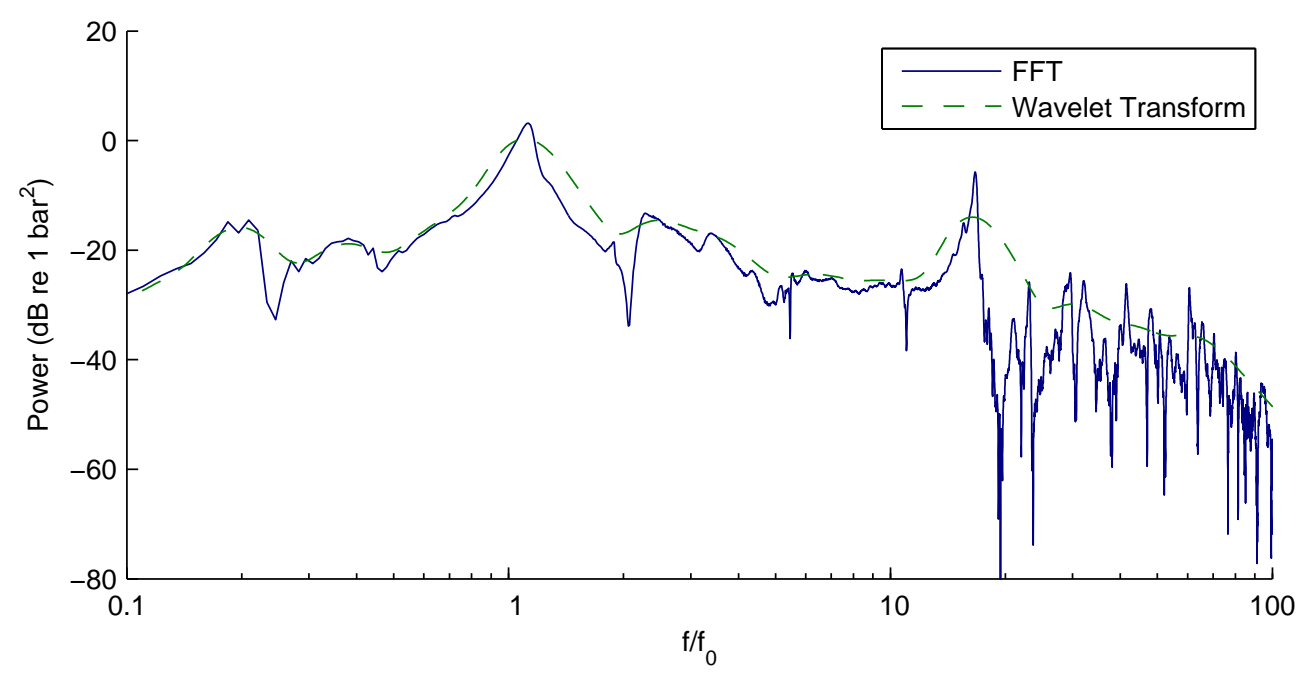

(a) 50 bar initial pressure

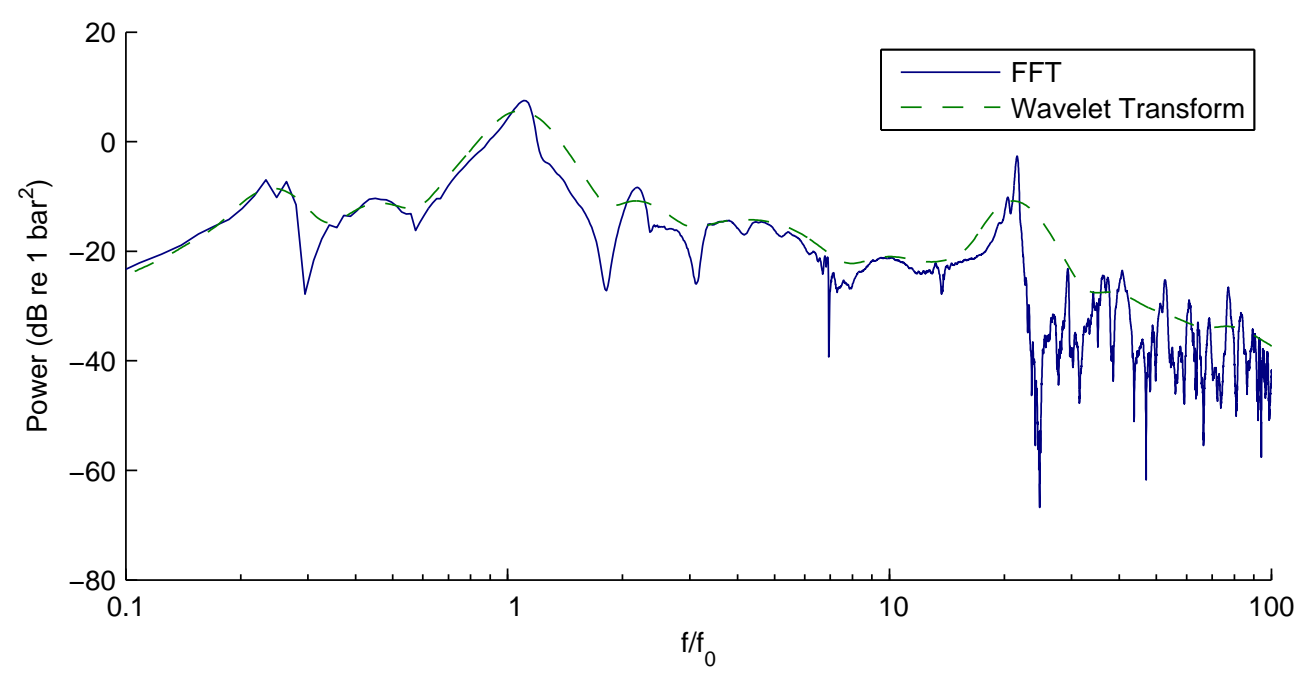

(b) 100 bar initial pressure

Figure 4.13: Comparison of PSDs from the wavelet transform and FFT. Both PSDs show the dominant bubble and reverberation frequencies clearly. Frequency is non-dimensionalised using Minneart's frequency, $f_{0}$, for an equivalent bubble at ambient pressure. 
The wavelet transform plot of the field pressure response for an initial airgun pressure of 50 bar (Figure 4.11a) shows the initial shock and subsequent bubble pulses generated every time the bubble collapses. Minneart's equation (Equation 4.1) gives the natural bubble frequency of an equivalent bubble as $62.2 \mathrm{~Hz}$ which compares favourably with the frequencies from the field pressure and accelerometer responses of 68 and $70 \mathrm{~Hz}$, derived from the wavelet transform and FFT, respectively. In both of these responses, the bubble pulsing frequency is the dominant frequency, as indicated by the magnitudes of the peaks in the PSDs. This is not the case for the wall pressure response which does not show the bubble frequency. This is due to phasing of the wall movement with the bubble pulses which is evident from the wall acceleration and is explained further in Section 4.3.6. The dominant frequency for the wall pressure response is $1033 \mathrm{~Hz}$ which can be found in the field pressure and accelerometer responses also. Frequencies of the standing waves stimulated in the tank can be estimated using [40]:

$$
f=\frac{c}{2} \sqrt{\left(\frac{l}{L_{x}}\right)^{2}+\left(\frac{m}{L_{y}}\right)^{2}+\left(\frac{n}{L_{z}}\right)^{2}}
$$

where $c$ is the speed of sound, taken as $1480 \mathrm{~ms}^{-1}, L$ is the tank dimension in the $x, y$ and $z$ directions, all taken as $1.2 \mathrm{~m}$, and $l, m$ and $n$ are the respective ordered integers for the characteristic frequency. When these values are taken as 1 , the fundamental threedimensional standing wave is calculated as $1068 \mathrm{~Hz}$. This equation assumes rigid walls, whereas the tank has one free surface and arguably slightly compliant walls; however, the calculated frequency compares favourably with the range of frequencies measured (1021- $1063 \mathrm{~Hz})$.

Similar attributes of the frequency analysis of the 50 bar airgun pressure are evident in that of the 100 bar airgun pressure. The equivalent natural bubble frequency according to Minneart's equation (Equation 4.1) is $49.2 \mathrm{~Hz}$. From the field pressure response, the frequency derived by the wavelet transform is $53 \mathrm{~Hz}$ and by the FFT is $54 \mathrm{~Hz}$. The frequency of the tank reverberation is again evident in all three responses. Table 4.1 summarises the bubble and reverberation frequencies derived by the wavelet transform and FFT for the three responses for both initial pressures.

\subsubsection{Hydrodynamic and Acoustic Pressure}

Felli et al. [22] note that the hydrodynamic and acoustic contributions to a pressure signal can be distinguished as only the acoustic contributions satisfy the linear wave equation and radiate as sound. The hydrodynamic pressure contributions radiate at a much smaller velocity and at a distance, disappear. The PSDs from wavelet analysis of 


\begin{tabular}{lcc}
\hline Initial Airgun Pressure & $50 \mathrm{bar}$ & $100 \mathrm{bar}$ \\
\hline Field pressure bubble frequency (wavelets) & $\mathbf{6 8 ~ H z}$ & $\mathbf{5 3 ~ H z}$ \\
Field pressure bubble frequency (FFT) & $\mathbf{7 0 ~ H z}$ & $\mathbf{5 4 ~ H z}$ \\
Wall pressure bubble frequency (wavelets) & Not evident & Not evident \\
Wall pressure bubble frequency (FFT) & Not evident & Not evident \\
Accelerometer bubble frequency (wavelets) & $\mathbf{6 8 ~ H z}$ & $\mathbf{5 1 ~ H z}$ \\
Accelerometer bubble frequency (FFT) & $70 \mathrm{~Hz}$ & $55 \mathrm{~Hz}$ \\
Field pressure reverberant frequency (wavelets) & $1021 \mathrm{~Hz}$ & $1032 \mathrm{~Hz}$ \\
Field pressure reverberant frequency (FFT) & $1036 \mathbf{H z}$ & $1061 \mathrm{~Hz}$ \\
Wall pressure reverberant frequency (wavelets) & $\mathbf{1 0 3 3 ~ H z}$ & $\mathbf{1 0 5 3 ~ H z}$ \\
Wall pressure reverberant frequency (FFT) & $\mathbf{1 0 3 8 ~ H z}$ & $\mathbf{1 0 6 2 ~ H z}$ \\
Accelerometer reverberant frequency (wavelets) & $\mathbf{1 0 3 2 ~ H z}$ & $1053 \mathbf{H z}$ \\
Accelerometer reverberant frequency (FFT) & $1038 \mathrm{~Hz}$ & $\mathbf{1 0 6 3 ~ H z}$ \\
Minneart's frequency & $62.2 \mathrm{~Hz}$ & $49.2 \mathrm{~Hz}$ \\
\hline
\end{tabular}

Table 4.1: Bubble and reverberant field frequencies obtained from the wavelet transform and FFT analyses. Bold face indicates dominant frequency.

the field pressure responses at varying distances from the airgun (fired at 100 bar) are plotted in Figure 4.14. The frequency of the pressure pulses produced by the bubble (at a non-dimensional frequency of 1) decrease in intensity with increasing distance, confirming the hydrodynamic contribution to the pressure response, while the frequency of the reverberant field (at a non-dimensional frequency of 20, or approximately 1000 $\mathrm{Hz}$ ) does not change significantly in intensity, which is reveals this response to be sound radiation.

\subsubsection{Wall Movement}

The movement of the steel plate is found through double integration of the wall acceleration. A cumulative error is evident in the velocity after the first integration and is removed by fitting a power function to the general error trend and subtracting it from the results.

The wall displacement is plotted with the field pressure, wall pressure and wall acceleration to show phasing for various conditions in Figures 4.15 and 4.16. For the displacement and acceleration data, the positive direction is outward from the centre of the tank. Figure 4.15 shows results for cases where the airgun is located at the centre of the tank and fired at 50 and 100 bar initial pressures. For these cases, the wall acceleration and field pressure are in phase with each other, while the relative wall movement is 180 degrees out of phase; i.e. the wall moves with the bubble pulsation. It is this outward movement of the wall when the pressure pulse arrives that results in the 


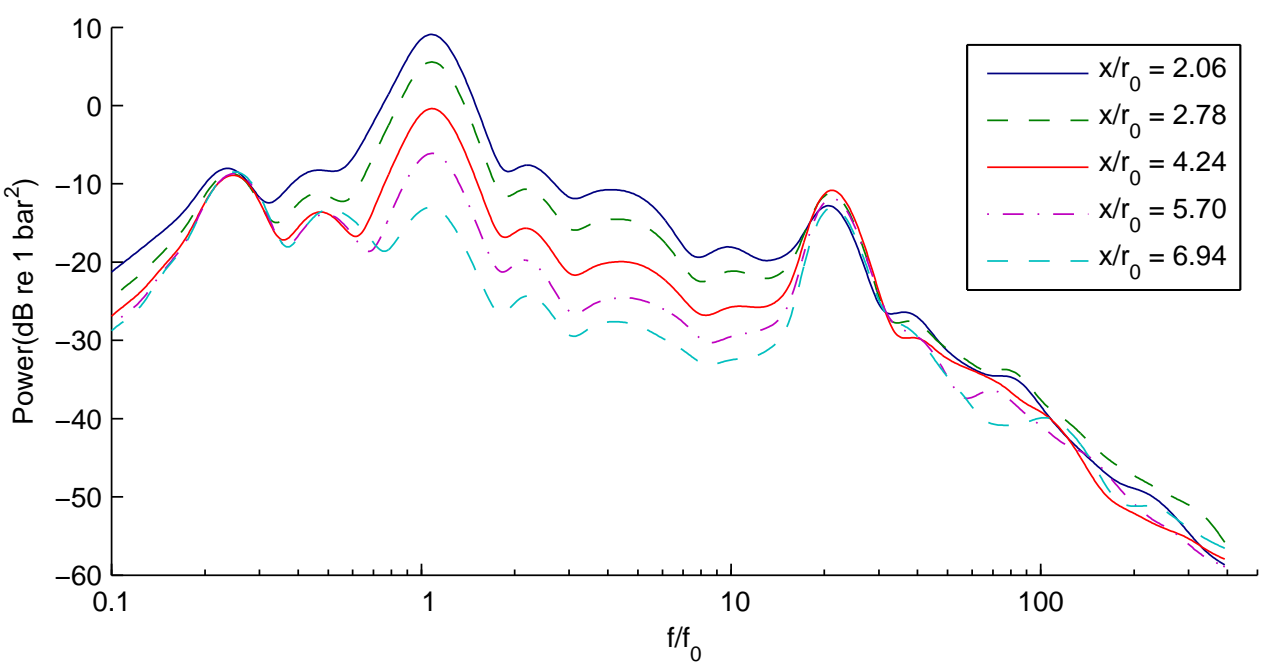

Figure 4.14: PSD from the wavelet transform for the field pressure responses at varying distances from the airgun fired at 100 bar initial pressure. The intensity of the bubble frequency (non-dimensional frequency of 1 ) decreases with distance, while the intensity of the frequency of the reverberant field (non-dimensional frequency of 20, or approximately 1000 $\mathrm{Hz}$ ) remains constant. This is consistent with the characteristics of the hydrodynamic and acoustic contributions to a pressure signal. Frequency is non-dimensionalised using Minneart's frequency, $f_{0}$, for an equivalent bubble at ambient pressure.

very small response of the pressure transducer to the bubble pulses (evidenced here and in the wavelet transforms in Figures 4.11 and 4.12). For the 50 bar initial pressure, the maximum outward wall displacement of the steel plate is $0.15 \mathrm{~mm}$ and the maximum inward wall displacement is $0.19 \mathrm{~mm}$. For the 100 bar initial pressure, the maximum outward wall displacement is $0.38 \mathrm{~mm}$ and the inward $0.59 \mathrm{~mm}$.

Figure 4.16 shows results for initial airgun pressures of 50 and 100 bar, when the airgun is fired $200 \mathrm{~mm}$ from the wall. For these cases, the wall pressure starts to respond to the bubble pulses and is slightly out of phase with the wall movement. A slight aberration in the regularity of the wall movement suggests the influence of the wall's natural frequency. The hydrophone is much further away from the airgun for these firings and as a result the response is largely degraded. The wall movement remains in phase with the bubble growth and collapse and has larger magnitudes than previous due to the proximity of the gun. The maximum outward and inward wall displacements for the 50 bar and 100 bar initial pressures respectively are $0.46 \mathrm{~mm}$ and $0.68 \mathrm{~mm}$ (50 bar) and $1.25 \mathrm{~mm}$ and $1.57 \mathrm{~mm}$ (100 bar). 


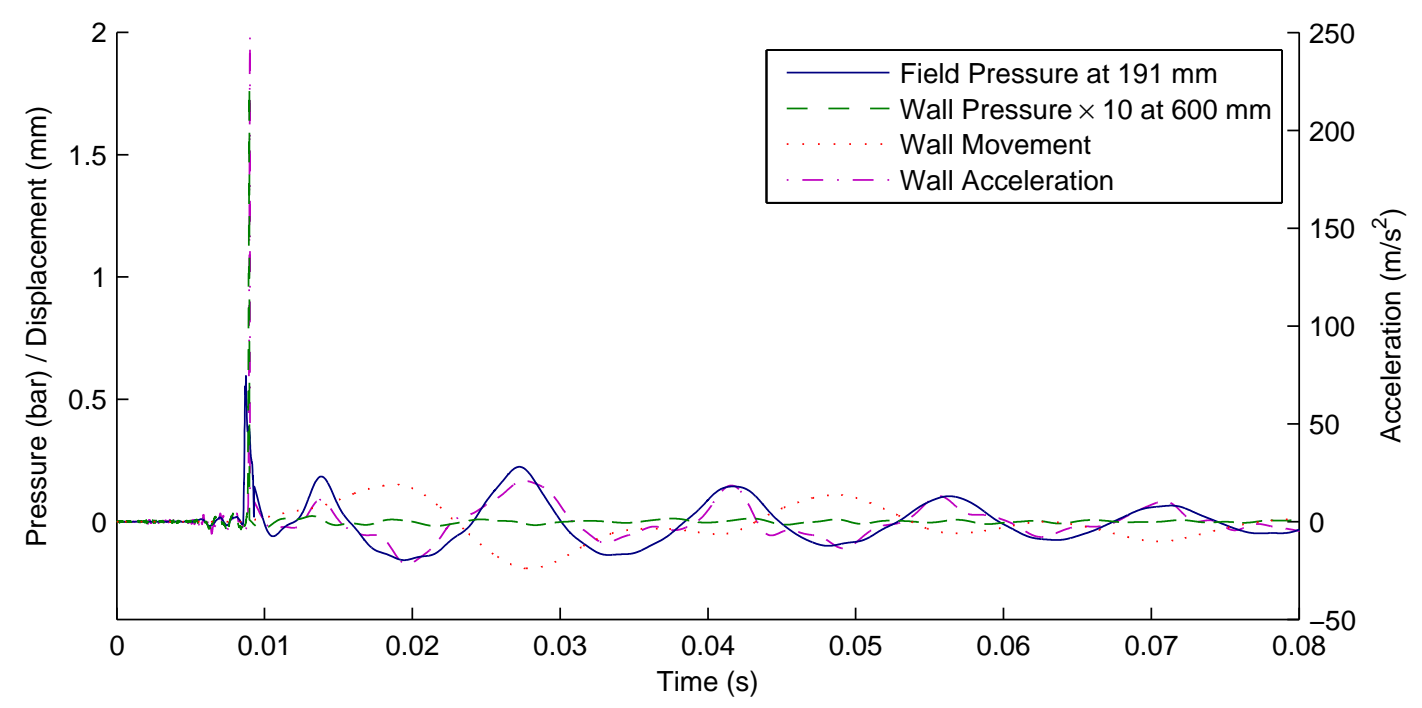

(a) 50 bar initial pressure

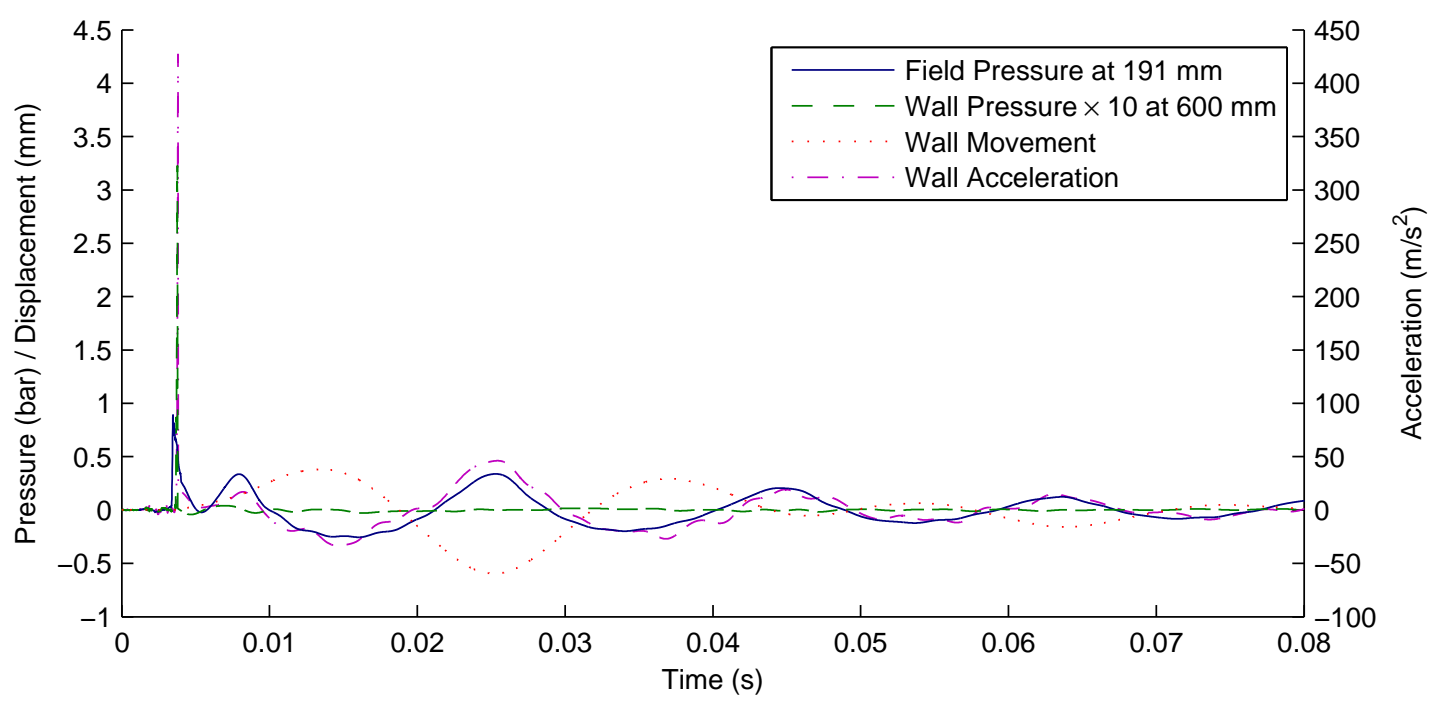

(b) 100 bar initial pressure

Figure 4.15: Field pressure, wall pressure, wall acceleration and wall movement for 50 and 100 bar initial airgun pressures. The airgun is located at the centre of the tank. The initial shock is felt at the wall but not the subsequent pressure pulses from the bubble collapses, due to the wall responding in phase to the bubble. 


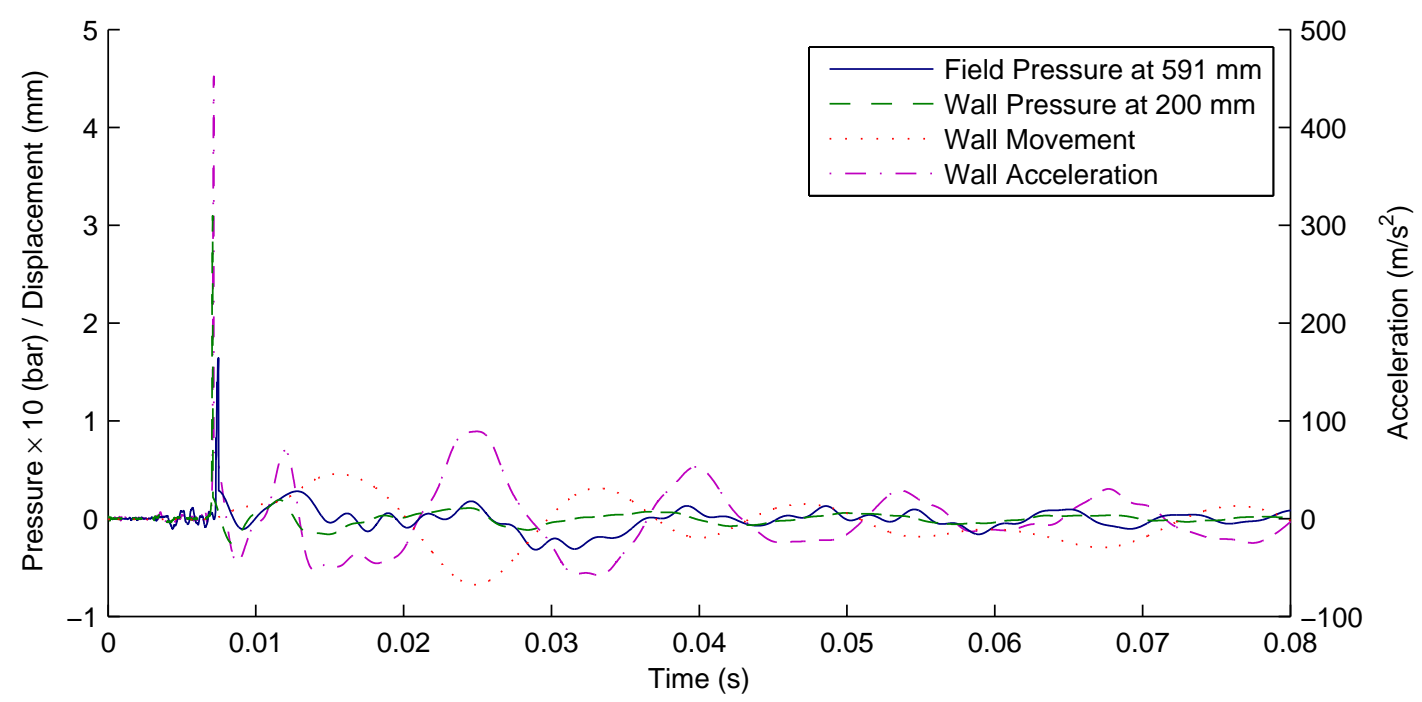

(a) 50 bar initial pressure

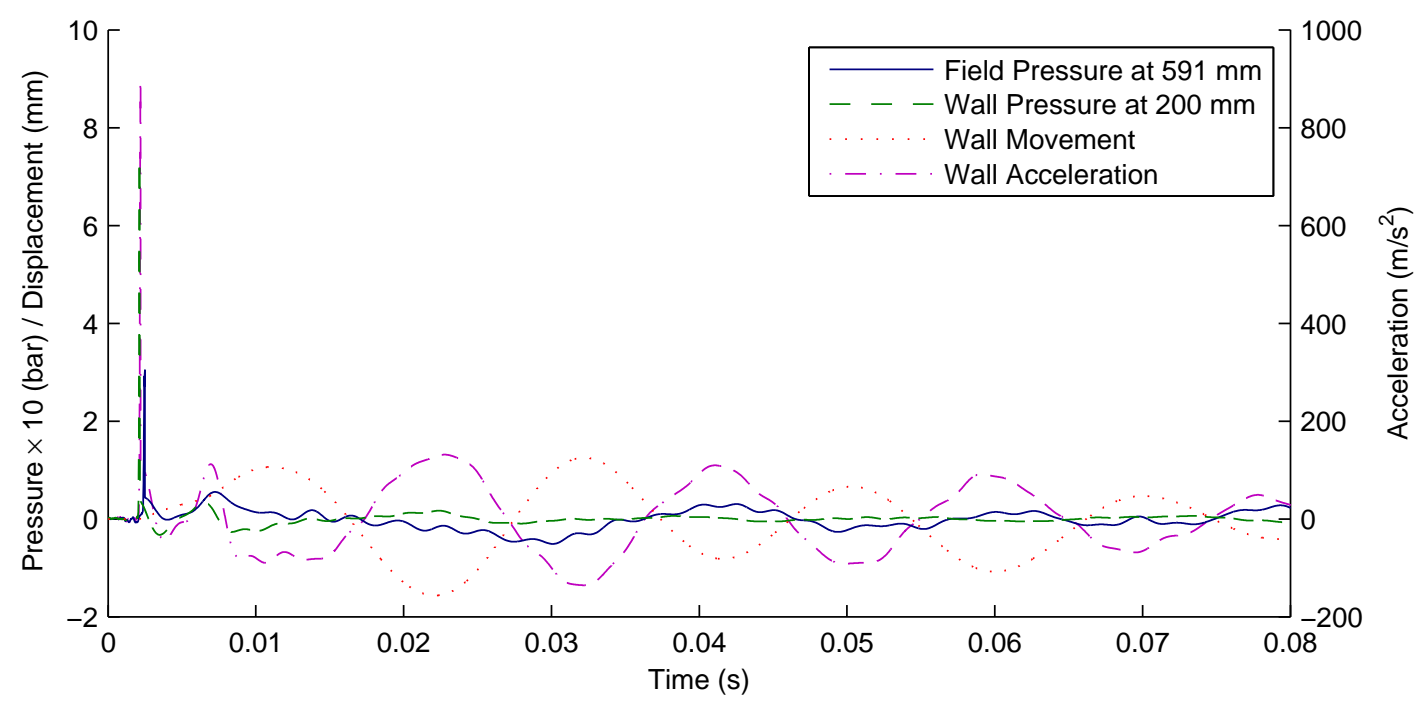

(b) 100 bar initial pressure

Figure 4.16: Field pressure, wall pressure, wall acceleration and wall movement for 50 and 100 bar initial airgun pressures. The airgun is located $200 \mathrm{~mm}$ from the steel wall. The initial shock is felt at the wall but very little is felt from the subsequent pressure pulses from the bubble collapses, due to the wall responding nearly in phase to the bubble. 


\subsubsection{Pressure with Distance}

The decay of the initial shock with distance has been obtained from three data sets: wall pressure with varying airgun distance, field pressure (fixed location in tank) with varying airgun distance and field pressure with varying hydrophone distance from the centrally located airgun. These are shown in Figure 4.17. A line of best fit shows the initial shock magnitude to vary proportional to $x^{-1.0785}$ for the 50 bar firings and $x^{-0.98098}$ for the 100 bar firings. This agrees well with theory which predicts that the acoustic pressure amplitude of a spherical wave decays inversely with increasing distance in the far field [40].

The decay of the pressure pulse generated by the first bubble collapse has also been obtained from the field pressure data (Figure 4.18). As shown in Section 4.3.4, the bubble pulses were not evident in the wall pressure response. The pulse from the bubble collapse decays at a greater rate than the $x^{-1}$ acoustic law, which is evidence that, as a result of taking measurements in the near field, the wave is composed of more than only acoustic contributions, as discussed in Section 4.3.5.

Field pressure results were also obtained for an initial airgun pressure of 100 bar at a horizontal standoff distance of $184 \mathrm{~mm}$ and varying vertical depths from $300 \mathrm{~mm}$ below the airgun port to $500 \mathrm{~mm}$ above the airgun port. At $500 \mathrm{~mm}$ above the airgun port $\left(z / r_{0}=-7.3\right)$, the hydrophone was at the free surface. It is evident that for a certain angle from the port the field pressure is reasonably constant, but outside this window the pressure decreases as shown in Figure 4.19.

\subsubsection{Interaction with Free Surface}

The interaction of the bubble with a free surface was tested by lowering the depth of the water in the tank progressively. This ensured the airgun remained in the plane of the field and wall pressure transducers. For each free surface standoff distance, $z_{f s}$, an average was taken of 10 filtered field pressure responses at a horizontal standoff distance of $184 \mathrm{~mm}$. The standoff distance is non-dimensionalised using the maximum radius, $R_{M}$, which is calculated as the radius of a sphere with the same volume as the airgun bubble, assuming it is an oblate spheroid (taken from the high-speed footage), not excluding the volume of the airgun. The change in frequency and magnitude of the bubble collapses at the different free surface standoff distances is clearly shown in Figure 4.20. When the bubble is close to the surface, the frequency increases. This is caused by the reduction in surrounding water mass, and hence fluid inertia, with reduced distance to the free surface [52]. The reduction in magnitude of the pressure pulses with free surface proximity can 


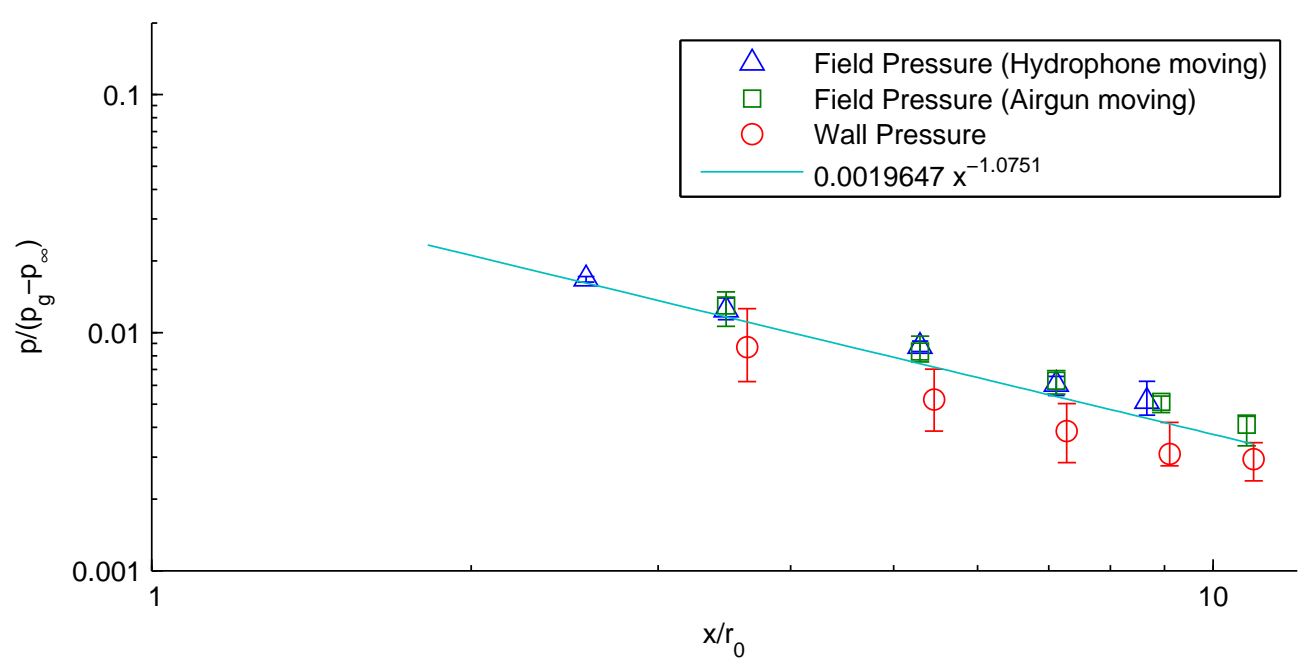

(a) 50 bar initial pressure

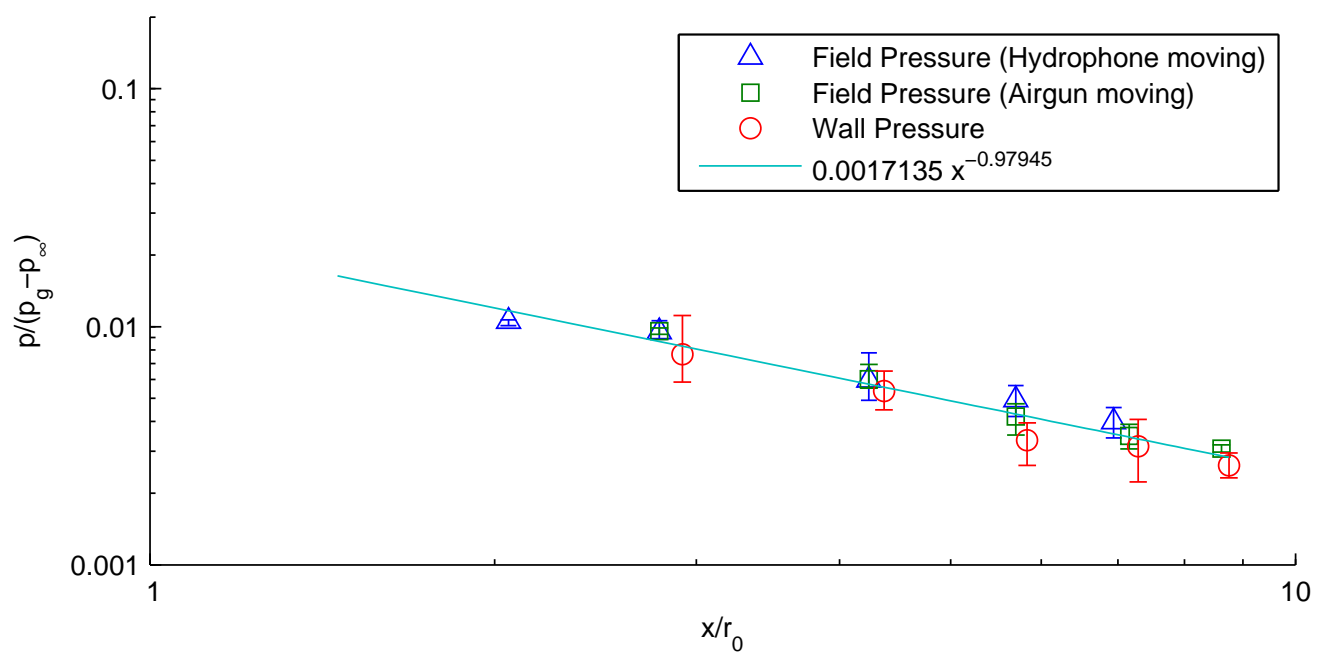

(b) 100 bar initial pressure

Figure 4.17: Pressure with distance of the initial shock for the airgun fired at 50 and 100 bar initial pressures. The pressure decays with $x^{-1}$, as predicted by acoustic theory. Pressure and standoff distance are non-dimensionalised using the difference between initial airgun pressure, $p_{g}$, and ambient pressure, $p_{\infty}$ and an equivalent bubble radius at ambient pressure, $r_{0}$, respectively. For this data, $r_{0}=0.0549$ and 0.0686 for 50 and 100 bar initial pressures, respectively. The 'error bars' represent the range of measured data (maximum and minimum measured values) obtained in the corresponding multiple firings. 


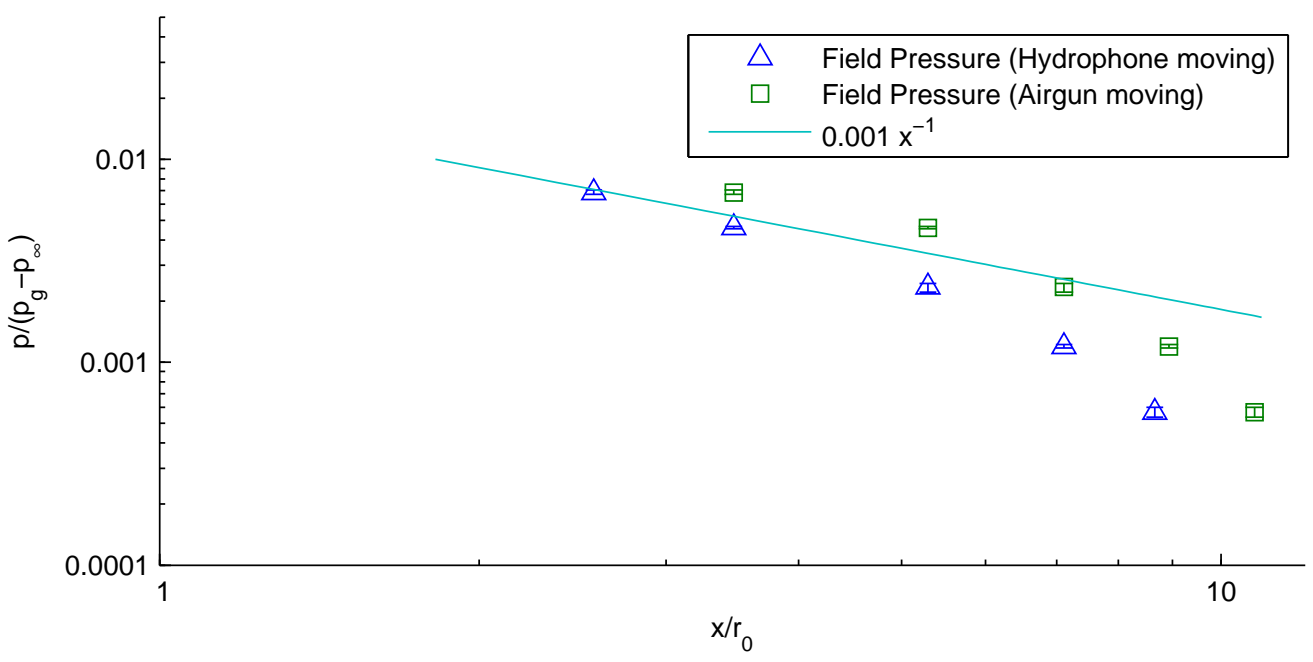

(a) 50 bar initial pressure

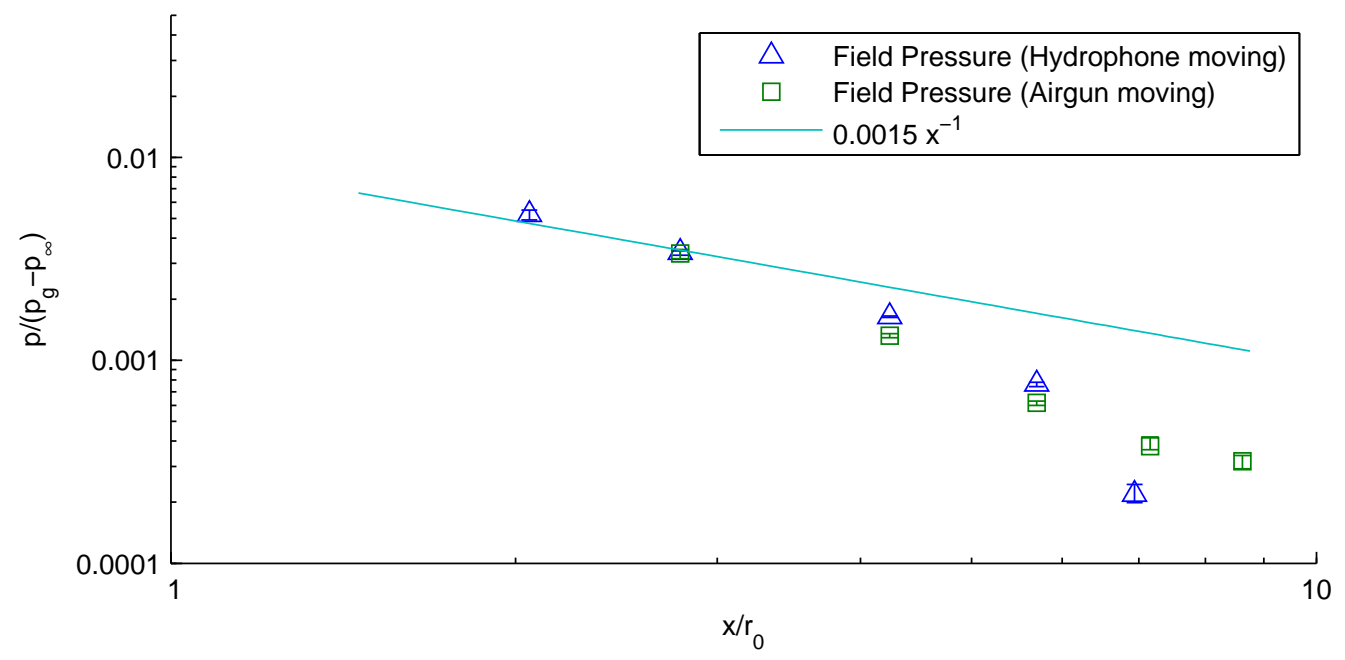

(b) 100 bar initial pressure

Figure 4.18: Pressure with distance of the pulse generated by the first bubble collapse for the airgun fired at 50 and 100 bar initial pressures. The decay rate is greater than $x^{-1}$ implying that the pressure wave has more than only acoustic contributions. Pressure and standoff distance are non-dimensionalised using the difference between initial airgun pressure, $p_{g}$, and ambient pressure, $p_{\infty}$, and an equivalent bubble radius at ambient pressure, $r_{0}$, respectively. For this data, $r_{0}=0.0550$ and 0.0686 for 50 and 100 bar initial pressures, respectively. The 'error bars' represent the range of measured data (maximum and minimum measured values) obtained in the corresponding multiple firings. 


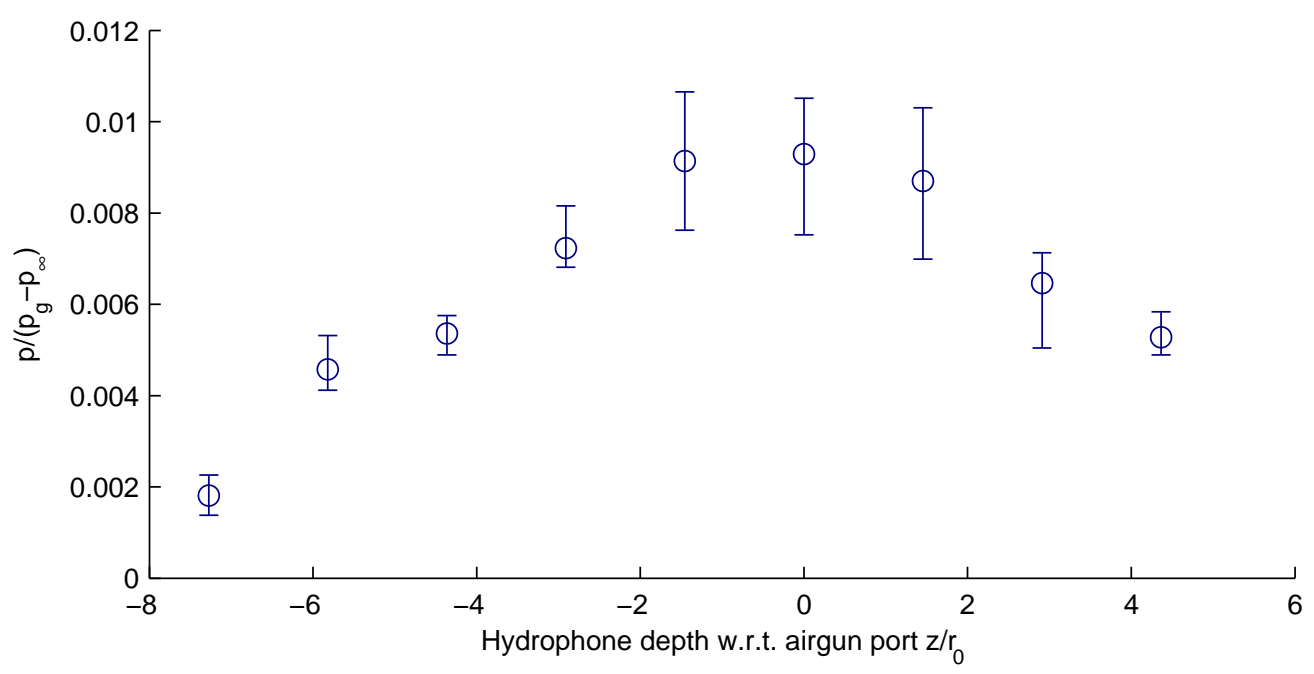

Figure 4.19: Initial shock pressure at different depths relative to the fixed-depth airgun fired at an initial pressure of 100 bar. The field is relatively constant over a small window, outside of which it decreases with increasing distance. Pressure and depth, $z$, are non-dimensionalised using the difference between initial airgun pressure, $p_{g}$, and ambient pressure, $p_{\infty}$, and an equivalent bubble radius at ambient pressure, $r_{0}$, respectively. For this data, $r_{0}=0.0687$. The 'error bars' represent the range of measured data (maximum and minimum measured values) obtained in the corresponding multiple firings.

also be attributed to the reduction of the surrounding water mass. Figure 4.21 shows the trend in the pressure pulses generated by the first bubble collapse with increasing standoff distance from the free surface. The initial shock is unaffected by the free surface proximity.

The average dominant frequency of 10 firings at each depth was determined from both FFT and wavelet analyses. The results of the change in bubble period with standoff distance are compared with those published by Tomita and Kodama [81] (for lasergenerated bubbles) and Hung and Hwangfu [35] (underwater explosion), and the image method of Best and Blake [5]. The bubble period, $\tau$, is non-dimensionalised with twice the Rayleigh collapse time [60]:

$$
\tau^{*}=\frac{\tau}{2 \tau_{\text {Rayleigh }}}
$$

and

$$
\tau_{\text {Rayleigh }}=0.915 R_{M} \sqrt{\frac{\rho}{p_{\infty}-p_{v}}}
$$

where $p_{v}$ is the water vapour pressure.

The trend of these results compares well with the published results; however, the actual bubble periods for the airgun are much longer than those predicted by the Rayleigh collapse time and therefore produce dimensionless periods much larger than those published. This could be attributed to several aspects. The Rayleigh collapse time is 


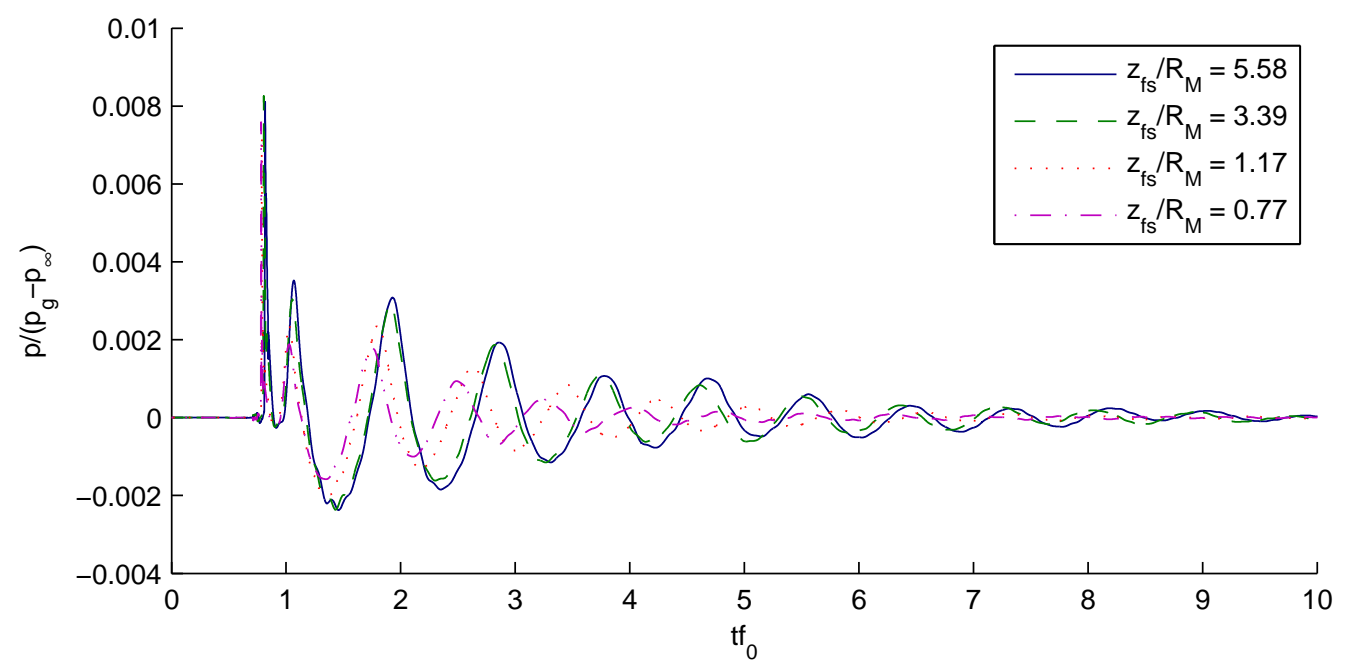

Figure 4.20: Averaged field pressure response at $184 \mathrm{~mm}$ for varying standoff distances from the free surface for 100 bar initial pressure. The initial shock is unaffected; however, the pressure pulses generated by the bubble collapses and the bubble period decrease with decreased standoff distance from the free surface. Pressure and time are non-dimensionalised using the difference between initial airgun pressure, $p_{g}$, and ambient pressure, $p_{\infty}$, and Minneart's frequency, $f_{0}$, for an equivalent bubble at ambient pressure, respectively.

derived for a vapour bubble, for which the internal pressure is maintained during collapse through mass transfer at the interface. In seismic airgun and underwater explosion bubbles, the presence of non-condensable gas, which increases in pressure as the bubble size reduces, slows the collapse time. The bubble produced by this seismic airgun is also not spherical, rather, it is toroidal in shape, with four distinct lobes at the port locations (as shown in Figure 4.5). The impact of this deviation in sphericity on the bubble period is not known. Also, as shown in [19] (Chapter 3), the effects of heat transfer across the bubble wall may increase the period of the seismic airgun bubble. If the firing chamber of a seismic airgun is at ambient pressure, as the expanding bubble cools, it will draw heat from the surrounding water, thus increasing its period. This is contrary to an underwater explosion bubble, which starts at extremely high temperatures, and is further cooled by the surrounding water as it expands, thus reducing its energy and the bubble period. 


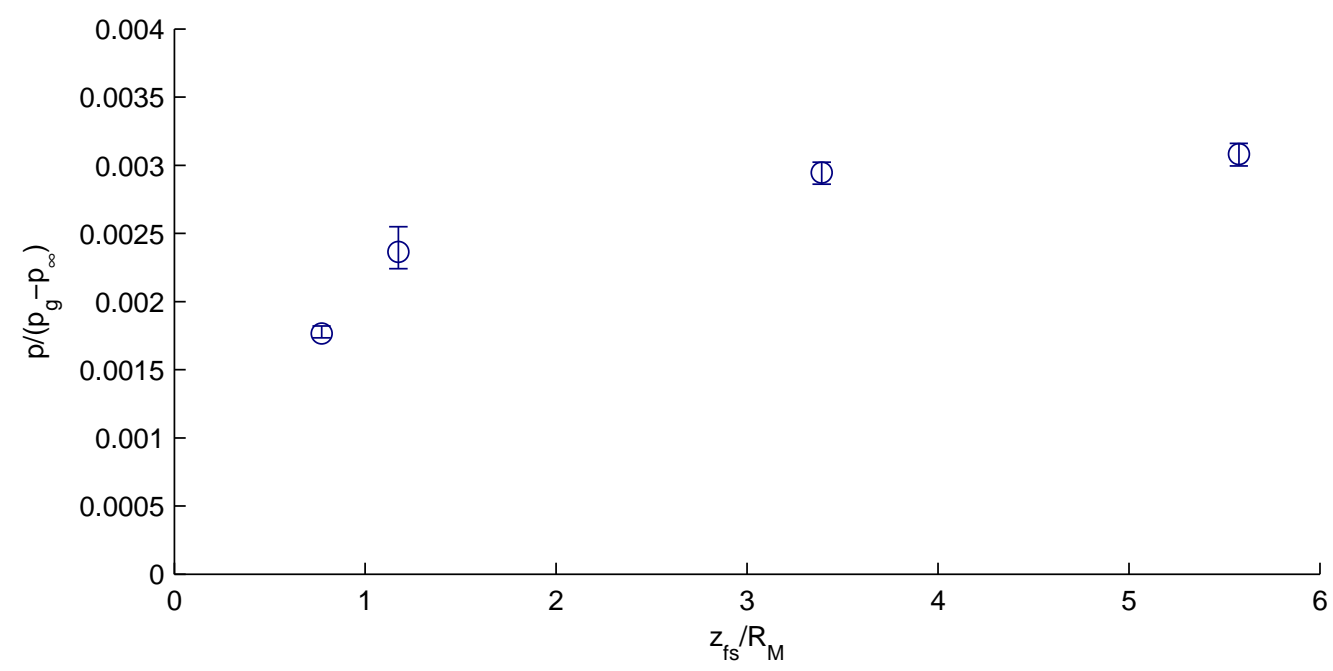

Figure 4.21: Pressure pulse at a horizontal standoff distance of $184 \mathrm{~mm}$ generated by the first bubble collapse, with respect to airgun standoff distance from the free surface for 100 bar initial pressure. The pressure pulse is obtained from the average of 10 firings and is nondimensionalised using the difference between initial airgun pressure, $p_{g}$, and ambient pressure, $p_{\infty}$. Free surface standoff distance, $z_{f s}$, is non-dimensionalised using an equivalent spherical maximum bubble radius, $R_{M}$, calculated as the radius of sphere with the same volume as the airgun bubble, assuming it is an oblate spheroid (taken from the high-speed footage), not excluding the volume of the airgun. The 'error bars' represent the range of measured data (maximum and minimum measured values) obtained in the corresponding multiple firings.

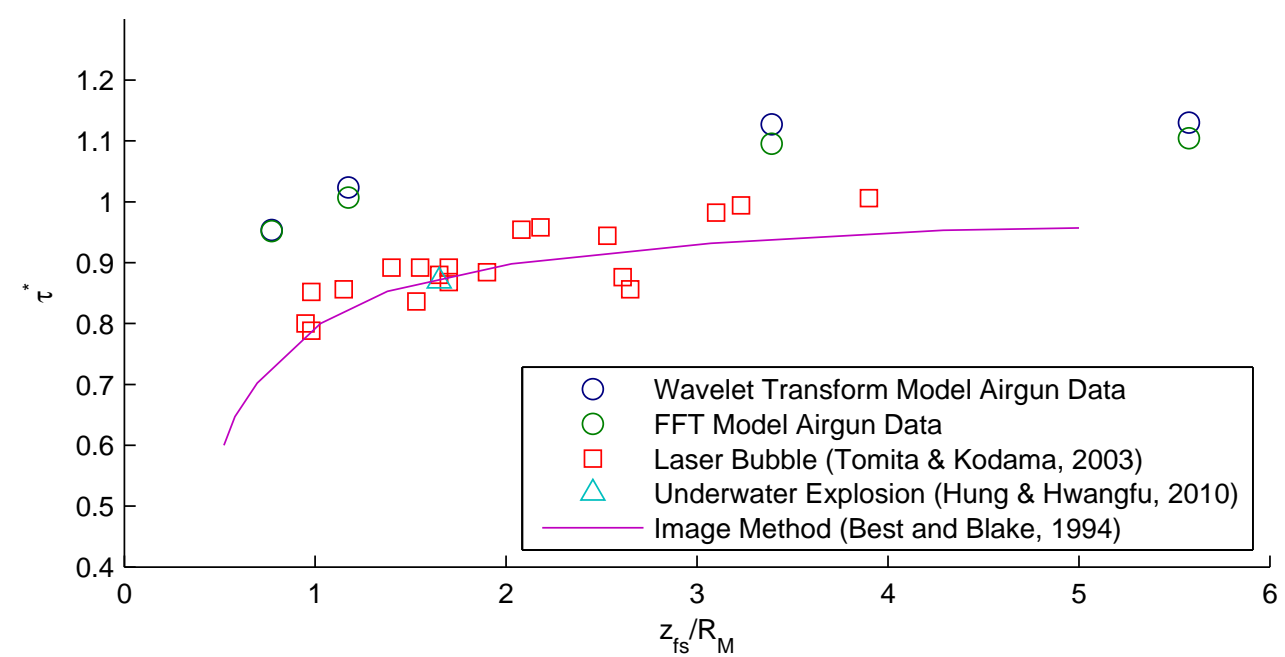

Figure 4.22: Bubble period with respect to airgun standoff distance from the free surface for 100 bar initial pressure. The bubble period is obtained from the average dominant frequency of 10 firings using both FFT and wavelet methods. The trend of the present results compares well with published data. The bubble period and free surface standoff distance are nondimensionalised with twice the Rayleigh collapse time and an equivalent spherical maximum bubble radius, $R_{M}$, respectively. The maximum radius is calculated as the radius of sphere with the same volume as the airgun bubble, assuming it is an oblate spheroid (taken from the high-speed footage), not excluding the volume of the airgun. 


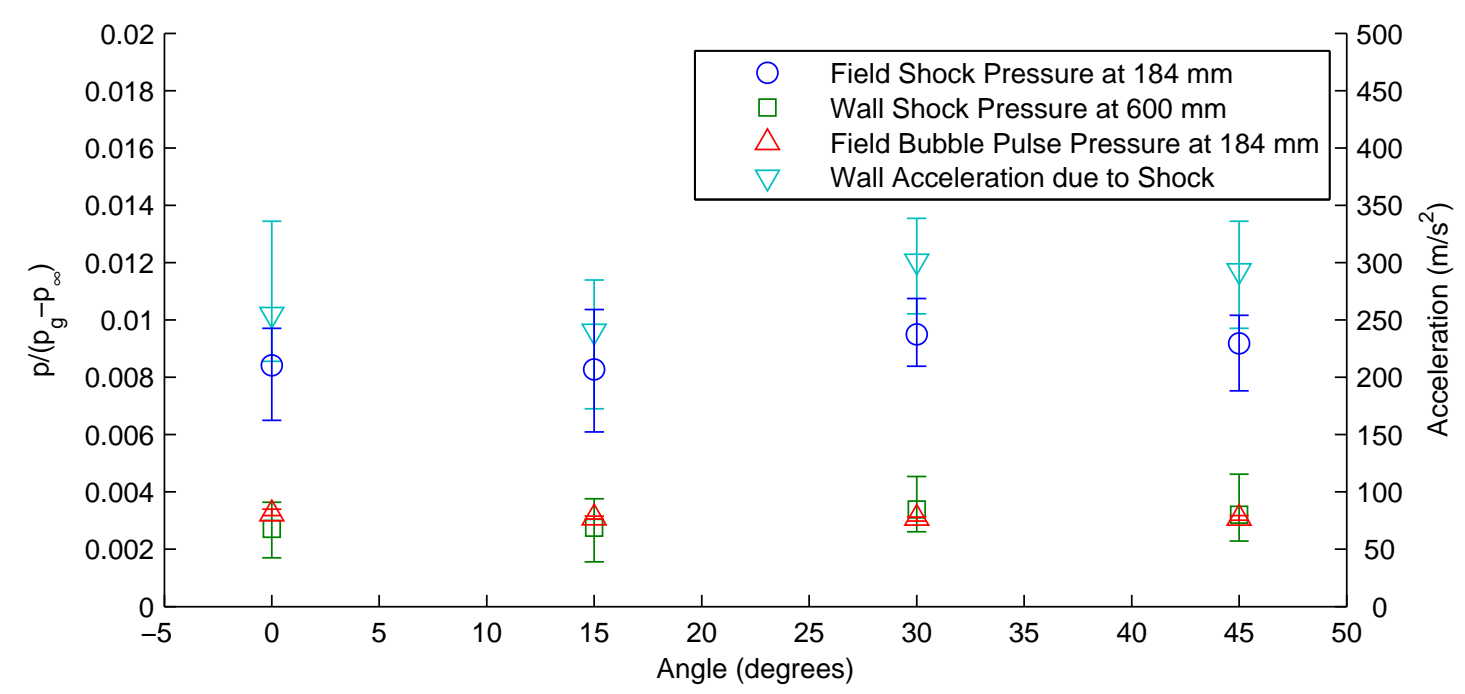

Figure 4.23: Directivity of the airgun when fired at 100 bar initial pressure. Results were obtained for one 45 degree sector due to symmetry of the ports. No significant difference in shock strength is noted beyond that due to shot to shot variability. Acceleration data has been scaled using $\frac{p_{g}}{100 p_{\infty}}$ to account for fluctuations in firing pressure. Pressure is non-dimensionalised using the difference between initial airgun pressure, $p_{g}$, and ambient pressure, $p_{\infty}$. The 'error bars' represent the range of measured data (maximum and minimum measured values) obtained in the corresponding multiple firings.

\subsubsection{Directivity}

Directivity of the scale airgun was tested by rotating the airgun body through 45 degrees in 15 degree increments. The field and wall mounted pressure transducers were located at $184 \mathrm{~mm}$ and $600 \mathrm{~mm}$ from the airgun, respectively. The airgun was located in the centre of the tank and fired at 100 bar. Acceleration data has been scaled using $\frac{p_{g}}{100 p_{\infty}}$ to account for fluctuations in firing pressure. No significant difference in directivity is noticed for the airgun, as shown in Figure 4.23.

\subsubsection{Scaling}

The presented results have been obtained with a model-scale seismic airgun fired at similar pressures to full-scale airguns. Solutions to the well-known Gilmore equation [27] show that bubble velocity is a function of firing pressure. The body dimensions and firing volume have been designed to maintain the relative scale with respect to the maximum bubble diameter of a full-scale airgun. Despite the pressure being the same for the model and full-scale airguns, the available energy for the bubble scales with respect to the firing volume. Heat transfer across the bubble wall and surface turbulence are identified to have a significant effect on the bubble dynamics of a seismic airgun and when considered 
together, are not terms which can be scaled easily. Further study is required to determine how the maximum radius, bubble period, shock strength and pressure fields scale with airgun size. The data set presented here provides a basis for future comparison with full-scale airgun data.

\subsection{Conclusion}

A laboratory scale seismic airgun has been built and used to study the pressure field generated when fired at two different pressures in an open top tank. The airgun was fired at varying standoff distances from a steel plate and from the free surface. Wavelet transforms and FFTs were used successfully to extract bubble and reverberant frequencies in the tank environment. For this airgun and tank arrangement, significant impact on the adjacent semi-compliant wall is only felt from the initial shock. The steel plate moves in phase with the bubble pulsation and hence the pressure felt at the wall from the bubble pulses is nearly non-existent for larger standoff distances. For closer standoff distances, a slight difference in phasing between the wall movement and the bubble pulsation resulted in a minimal pressure response at the wall due to the bubble collapses. The strength of the initial shock was found to decrease inversely with respect to distance as expected; however, the strength of the pressure pulse generated by the first bubble collapse did not, showing the hydrodynamic, and hence short range, nature of the bubble's pressure field. The period of the bubble interacting with the free surface follows the same trends as non-airgun produced bubbles, although the dimensionless periods were larger than those for explosion and laser-generated bubbles. These differences are attributable to differences in bubble contents, topology and thermal considerations. The results presented here provide data which can be used in conjunction with full-scale airgun results for comparison with future numerical predictions.

\section{Acknowledgements}

This work has been funded by the Maritime Platforms Division of the Defence Science and Technology Organisation (DSTO) and the authors wish to acknowledge the support of Dr Stuart Cannon and Mr Warren Reid.

The authors also wish to acknowledge the support of Dr Bryce Pearce and invaluable technical assistance of Mr Robert Wrigley from the Australian Maritime College Cavitation Research Laboratory. 


\title{
5
}

\section{Bubble Dynamics of a Seismic Airgun}

This chapter has been submitted for publication with Experimental Thermal and Fluid Science. The contributing authors are: K.L. de Graaf, P.A. Brandner and I. Penesis.

\begin{abstract}
The dynamics of the bubble generated by a four port model-scale seismic airgun in an open top tank are investigated. The airgun is fired at 50 and 100 bar and bubble images are captured with low- and high-speed photography. Forward- and back-lit photography allow close observation of the phenomena at the gas-water interface, and measurement of the radial growth, respectively. The development of the Rayleigh-Taylor Instability on the bubble's surface is identified. Field pressure measurements, synchronised in time with the radial bubble growth, are presented, and features of the emitted pressure signature are associated with the physical bubble dynamics. The experimental data is compared with an analytical prediction based on the Gilmore equation and good agreement is found for bubble radius, bubble period and the pressure pulse emitted at bubble collapse. The initial shock wave and first maximum velocity were over-predicted by this method.
\end{abstract}

\subsection{Introduction}

Seismic airguns have been developed for the generation of seismic pulses for ocean seabed geophysical exploration. Research on airguns has primarily been driven by the development of these geological surveying techniques and has focused on quantifying the initial shock wave emitted and using the interaction of multiple airguns to minimise the 
following bubble pulses. This primarily involves pressure measurements and development of numerical and empirical methods.

Bubble dynamic models such as the Gilmore equation and Rayleigh equation (or Rayleigh-Plesset equation, if surface tension and viscosity are included) are commonly used as the underlying basis for models of seismic airgun bubbles despite their deviation from a strictly spherical form. The dynamics and behaviour of the bubble produced by an airgun are not particularly well understood as obtaining high-speed photography of full-scale airguns is difficult when considering water quality and domain size.

The attributes of an airgun are also suitable for use in shock testing ships. Several navies are developing methods for this application and the Australian Government Defence Science and Technology Organisation (DSTO) has completed two trials with commercial SERCEL airguns. These trials measured the pressure field produced by the airgun and its effect on a scale model hull section $[12,71]$. A frame from high-speed video footage (provided by SERCEL) of the bubble produced by a full-scale airgun is shown in Figure 5.1 .

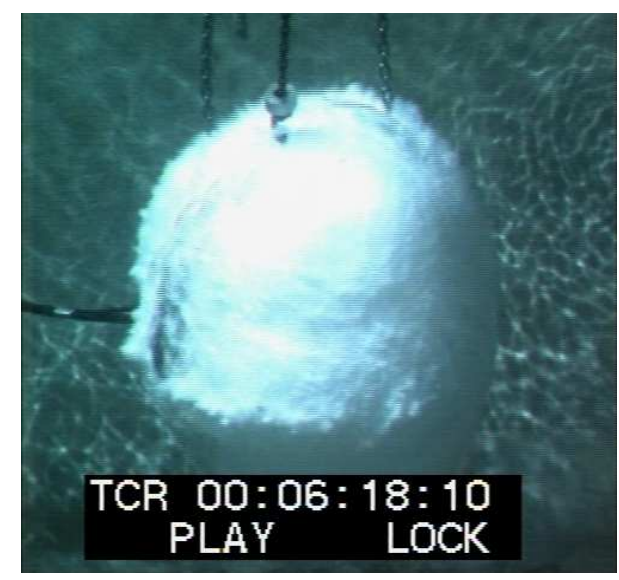

Figure 5.1: Single frame of video footage provided by SERCEL of GGun150 fired at 138 bar (2000 psi).

Studies on the bubble formed by an airgun have been presented by Bungenstock [6] and Langhammer and Landrø [45]. Bungenstock presents high-speed photography of the airgun bubble and discusses bubble damping with a view to suppression of the bubble that follows the initial shock (which is desirable in geophysical applications). The airgun studied by Bungenstock had a volume of $163 \mathrm{~cm}^{3}$ and was fired at 150 bar. Langhammer and Landrø studied a $26 \mathrm{~cm}^{3}$ BOLT 600B airgun fired at 100 bar, also using high-speed photography. The presence of water droplets or fog within the bubble was suggested as an explanation of the differences between modelled and measured signatures from airguns. Predictions of the bubble produced by a seismic airgun have been made by 
Ziolkowski [89, 90], Shulze-Gattermann [75], Johnston [37], Laws et al. [51], Li et al., [53] and de Graaf et al. [19]; however, uncertainties in the damping mechanisms still exist.

Unlike classical bubble work, where an initially spherical bubble is generated, the initial dynamics of a seismic airgun bubble are controlled by the impulsive release of gas through ports. Very little work has been published on impulsive, transient gas jets in liquids. Past work is focused on continuous jets and the subsequent dynamics and instabilities at varying flow rates, for example $[9,14,87]$.

A laboratory scale airgun and test facility, developed at the Australian Maritime College (AMC), has been used with low- and high-speed photography to study the dynamics of the air bubble generated. The photographs captured are of higher resolution than those previously presented in literature and allow the bubble physics to be explained in greater detail. Insight is given into the dynamics of a transient impulsive gas jet in water. Bubble growth measurements are presented with simultaneous field pressure measurements, and are compared with the analytical model developed in [19] (Chapter $3)$.

\subsection{Experimental Setup}

\subsubsection{Laboratory Scale Airgun}

A laboratory scale airgun has been designed based on a typical four port airgun. It has a firing volume of $14.5 \mathrm{~cm}^{3}$ and can be pressurized up to 100 bar from a standard dive bottle. Major external dimensions of the airgun are shown in Figure 5.2. The cylindrical airgun body has four $20 \mathrm{~mm}$ wide by $8 \mathrm{~mm}$ high ports through which the air is released, and is fitted to a pipe of equal outside diameter, as shown in Figure 5.3.

The operation of the model-scale airgun is based on the full-scale varieties and uses a pressure differential across two ends of a flanged shuttle to rapidly release a volume of compressed air, which forms the bubble. When the firing chamber is charged, the shuttle is held in place by the pressure difference acting on the slightly larger upper flange (Figure 5.3a). A central control rod drilled with small air passages is then moved upwards to expose the back of the upper flange to the firing pressure (Figure 5.3b). This results in the net force of the compressed air acting on the lower flange, immediately opening the shuttle and releasing the air through the four ports (Figure 5.3c). Once the air is exhausted, a spring returns the shuttle to its closed position and the control rod is then reset. 


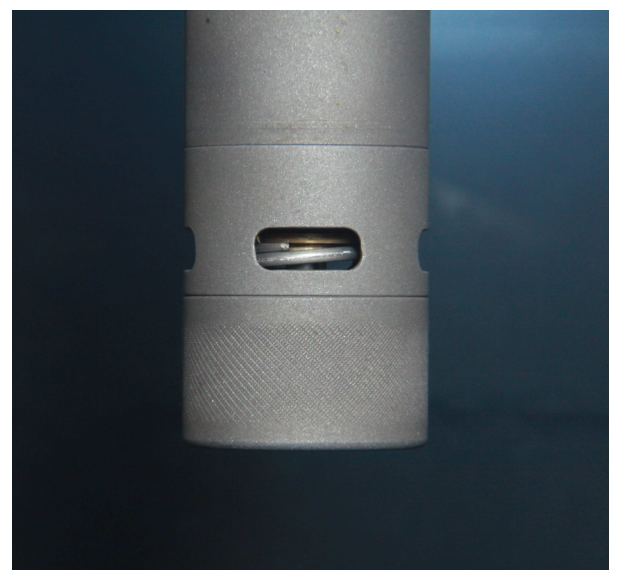

(a) Image of model-scale airgun

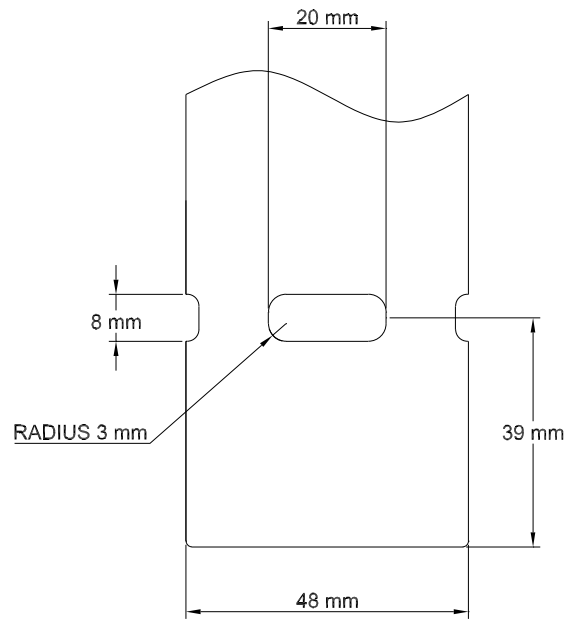

(b) Major external dimensions of the model-scale airgun

Figure 5.2: Model-scale airgun.

\subsubsection{Testing Tank and Equipment}

Experiments were carried out in the AMC Cavitation Research Laboratory. The airgun was suspended vertically in the centre of a $1.728 \mathrm{~m}^{3}$ open top water tank with dimensions of $1.2 \mathrm{~m} \times 1.2 \mathrm{~m} \times 1.2 \mathrm{~m}$. The tank is constructed with one $16 \mathrm{~mm}$ stainless steel plate side and the remaining three sides and base of $50 \mathrm{~mm}$ clear acrylic. The arrangement of the tank is shown in Figure 5.4. High-speed photography was taken using a LaVision High-Speed Star 5 camera with a Nikon Nikkor 55 mm F-1.4 lens controlled using DaVis 7.2.2 software. The camera was set up to film through an optical glass (N-BK7) viewing window in one of the acrylic sides. For these recordings, the camera was operated at 3 $\mathrm{kHz}$ with a resolution of $1024 \times 1024$ pixels, and a spatial resolution of approximately $0.29 \mathrm{~mm} /$ pixel. Additional close-up high-speed photography of the initial jet of released air was taken using a Nikon AF micro Nikkor $105 \mathrm{~mm}$ lens with the camera operated at 20 $\mathrm{kHz}$ and a resolution of $512 \times 256$ pixels. The spatial resolution was approximately 0.08 $\mathrm{mm} /$ pixel. Two dedocool lights, a dedolight 650 floodlight and two generic spotlights were required for adequate lighting. In addition to forward-lit photography, back-lit (shadowgraph) photography was also taken, filmed at the same two speed and resolution combinations. A $3 \mathrm{~mm}$ sheet of white opaque acrylic was used as a light diffuser with the dedolight 650 floodlight for the shadowgraph photography. The foward-lit photography allowed qualitative analysis of the bubble dynamics and structure, while back-lit photography enhanced the bubble surface/edge contrast to facilitate quantitative measurements of the bubbles' radius, velocity and acceleration. A Brüel \& Kjær (B\&K) Type 8103 hydrophone was suspended in the tank and a wall mounted PCB 112A21 


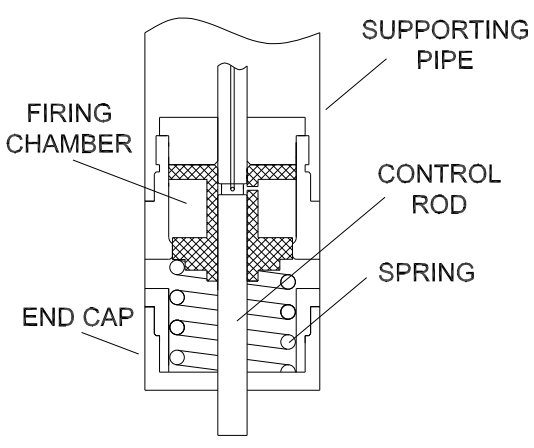

(a)

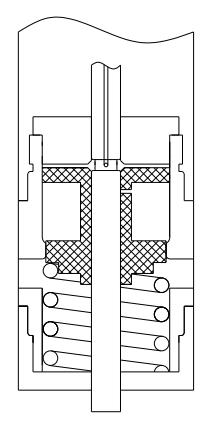

(b)

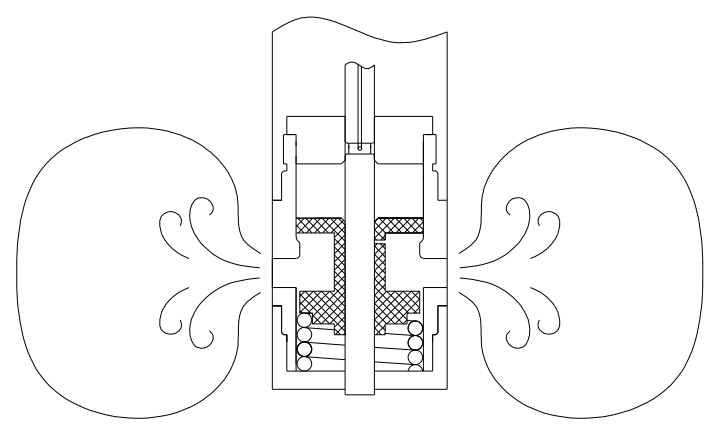

(c)

Figure 5.3: Illustration of the model-scale airgun firing in three stages: (a) pressurised chamber under equilibrium; (b) equalisation of pressure across upper flange to fire shuttle and (c) release of air through ports to form bubble.

pressure transducer and B\&K Type 4507 B004 Deltatron accelerometer were located on the steel wall. The hydrophone was used with a B\&K 2692 signal conditioning amplifier and the wall mounted pressure transducer and accelerometer were used with a PCB Piezotronics 482C signal conditioner. The pressure and acceleration data were simultaneously recorded at $100 \mathrm{kHz}$ using a National Instruments PCI-4472 simultaneous sample and hold acquisition card. When the airgun was fired, a trigger pulse via a variable delay (Thurlby Thandar Instruments TGP 110) was used to initialise data recording from the sensors and camera such that all results were time synchronised. The initial airgun pressure and ambient pressure were also recorded. The schematic in Figure 5.5 shows the overall data acquisition setup; for further detailed description of this equipment, refer to [17] (Chapter 4).

Higher resolution $(4752 \times 3168$ pixels, $0.05 \mathrm{~mm} /$ pixel $)$ still images were also taken at intervals throughout the bubbles' cycle using an EOS 50D Canon SLR camera with a Canon EF 24-70 mm Zoom lens. The camera exposure was controlled using a triggered stroboscopic flash (DRELLO 1018/LE4040), timed for various points in the bubble cycle using the variable delay. Despite the controllable delay between the airgun firing and strobe illumination, the slightly inconsistent timing (in the order of $1 \mathrm{~ms}$ ) of the actual air release caused some difficulties in obtaining images at specific instances in time. The analysis of the bubble dynamics has been done in reference to both high-speed images (for consecutive image sequences) and the higher resolution still camera (low-speed) images. A non-dimensional time, $t^{*}$, is given for each image with respect to the average 


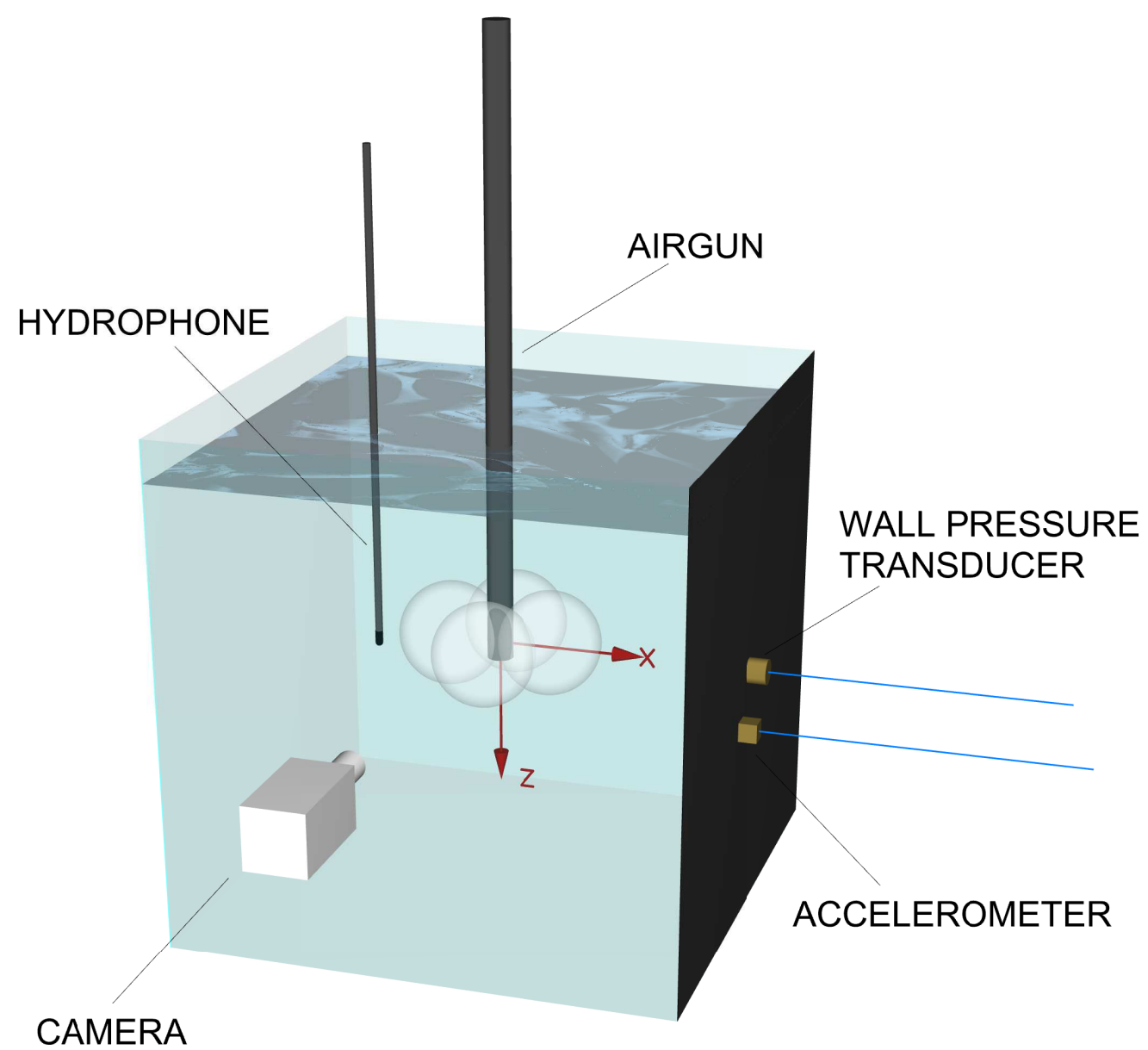

Figure 5.4: Experimental Setup. The $1.2 \mathrm{~m} \times 1.2 \mathrm{~m} \times 1.2 \mathrm{~m}$ tank has one $16 \mathrm{~mm}$ stainless steel plate side, and three sides and a base of $50 \mathrm{~mm}$ clear acrylic. The airgun and a hydrophone are suspended vertically from a rail spanning the tank which permits their positions to be independently adjusted vertically and horizontally. The wall pressure transducer is located at the centre of the stainless steel plate with the accelerometer adjacent. Improved optical access for the low- and high-speed photography is via an optical glass (N-BK7) insert in one acrylic side (not shown). 


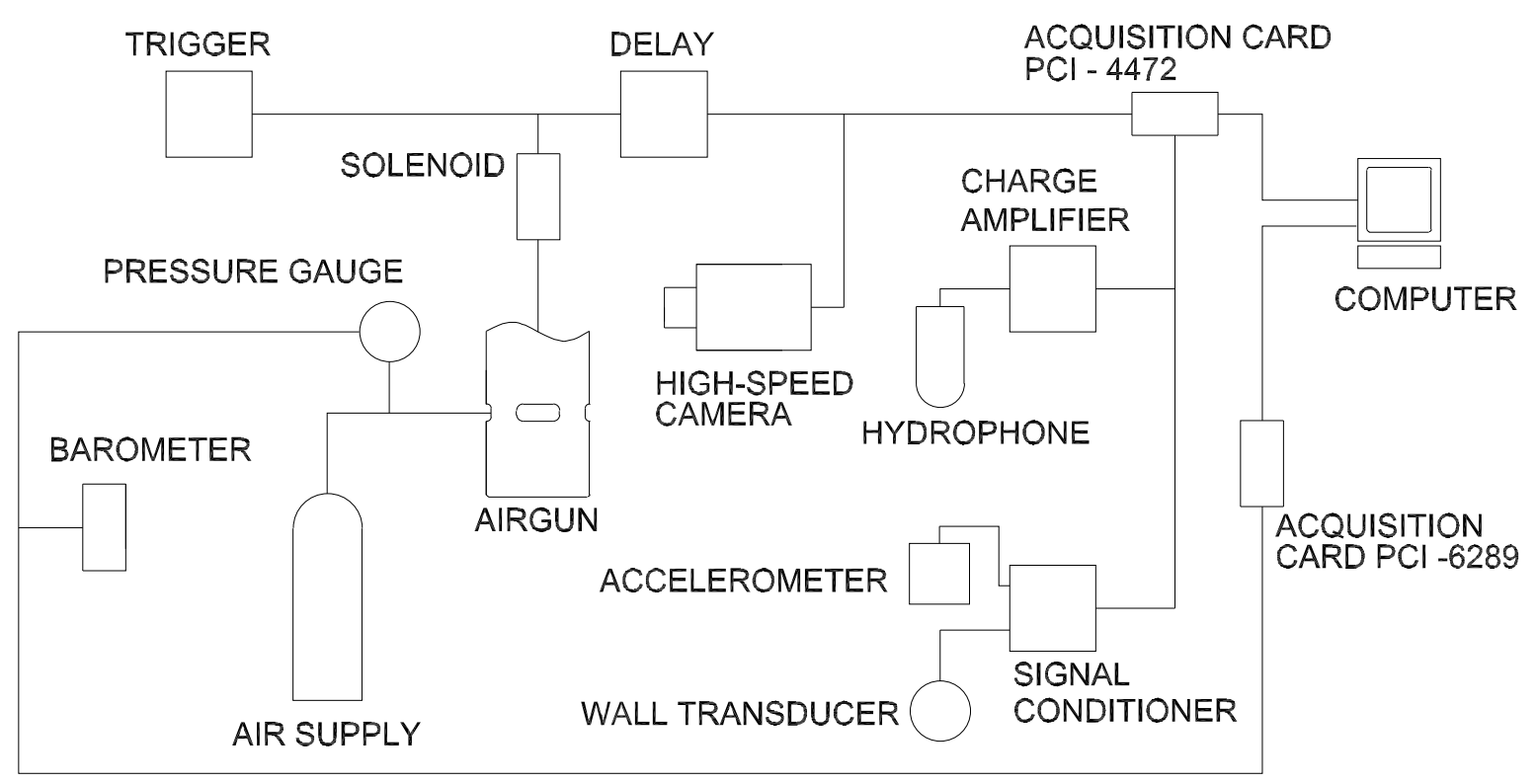

Figure 5.5: Schematic showing the setup of the data acquisition equipment.

first bubble period, $\tau_{1}$, taken from high-speed photography:

$$
t^{*}=\frac{t}{\tau_{1}}
$$

\subsection{Results and Discussion}

\subsubsection{Bubble Dynamics}

Various stages in the airgun bubble cycle are shown in a sequence of images in Figure 5.6. The bubble radius derived from high-speed photography (see Section 5.3.3) and the field pressure measured at a standoff distance of $184 \mathrm{~mm}$ from the airgun axis are shown in Figure 5.7. This figure also shows the times at which the images in Figure 5.6 occur in the bubble cycle. Both figures show how the bubble undergoes several rebounds before breaking up into a cloud of bubbles. The field pressure response in Figure 5.7 has been filtered after the time at which the reflection of the initial shock reaches the sensor, to preserve the transient shock while extracting the bubble pulsing frequency. Further details are given in [17] (Chapter 4).

For a full-scale airgun, the four jets of air emitted after firing appear to make a single bubble at the first maximum, as shown in Figure 5.1. This is not the case for the modelscale airgun bubble for which, as shown in Figure 5.6e, four distinct bubbles are still evident at the first maximum. 
The bubbles produced by the 50 and 100 bar initial airgun pressures behave similarly, with the dynamics and structures formed by the bubble from the 100 bar initial pressure being more evident and defined. The following description applies to the bubbles generated by both initial pressures, though the images shown are all for the 100 bar initial pressure case.

When the airgun is fired, the initial bubble interface has a rough and uneven texture, as shown in Figure 5.6a. Figure 5.8 shows selected frames from the $20 \mathrm{kHz}$ photography that capture the release of air after firing at an initial pressure of 100 bar. Figures $5.8 \mathrm{a}-$ $5.8 \mathrm{f}$ show each frame taken of the initial stage of the pressurised air issuing through the ports. The cavity around the periphery of the port in Figures $5.8 \mathrm{~b}-5.8 \mathrm{f}$ is potentially cavitation (vapour formation) caused by the shear stresses [38] or vortex core created by the impulsive liquid flow. The first signs of air release are evident at the top of the port as the shuttle moves down and the bubble growth encompasses any vapour formation. As the bubble grows, the velocity of the issuing air at the centre of the port is higher than that at the side, which is glassier in appearance, as shown in Figures $5.8 \mathrm{~g}$ - 5.8j. These figures, and Figure 5.6a, show the regularity of the surface perturbations which suggest the formation of surface instabilities. This is potentially the RayleighTaylor Instability (RTI), which forms at the interface of two fluids of different densities when the lighter fluid accelerates the denser. These perturbations are not given any opportunity to amplify due to the dominating effect of the developing gas turbulence during bubble growth, as evidenced by the clearly turbulent nature of the bubble surface in Figure 5.6c.

The Reynolds Number (Re) of the airflow issuing through the port has been estimated based on velocity from compressible flow theory, an average port dimension and properties of the compressed air. This gives $R e$ in the order of $1 \times 10^{7}$ for the 100 bar initial pressure which is well past the transition to turbulent flow. The velocity of the airflow through the port based on compressible flow theory is $200 \mathrm{~ms}^{-1}$, while the velocity of the emerging bubble taken from high-speed photography is approximately 20 $\mathrm{ms}^{-1}$. The stagnation of the emerging jet against the growing bubble interface leads to turbulence generation and jet break up which is responsible for the larger scale surface perturbations evident in the later stages of the initial bubble growth as shown in Figures $5.6 \mathrm{c}$ and $5.6 \mathrm{~d}$.

After the initial bubble formation, a secondary jet penetrates the bubble interface to form a second structure, as shown by the annular discontinuity around each of the four bubbles (only three are visible) in Figure 5.6b. Figure 5.9 shows an image sequence of the jet penetration captured with high-speed photography. [The jetting is clearly evident 
in the movie supplied]. The effect of this phenomenon is reflected in the field pressure and quantitative bubble growth measurements, identified in Figures 5.12 and 5.13. This is discussed further in Section 5.3.3.

As the jet velocity slows, the bubble continues to grow due to gas expansion (Figures $5.6 \mathrm{c}, 5.6 \mathrm{~d})$, and its rough surface becomes more glassy and smooth with turbulence dissipation, until the maximum radius is reached (Figure 5.6e). At the bubble maximum, the remnant structure formed by the penetrating jet is just visible and as the bubble collapses, the edges of this structure form the initial sites for the growth of the classical re-entrant jet which forms due to the proximity of the airgun body (Figure 5.6f). [This is also evident in the movie supplied]. An image sequence for the formation of this reentrant jet is shown in Figure 5.10. For the 50 bar initial pressure, the re-entrant jet is not as large in diameter.

The bubble rebounds with smaller glassy bubbles extending from the main bubble body (Figure 5.6g). These are attributed to the RTI. It is shown in Figure 5.13 that the interface acceleration is greater than zero (and therefore susceptible to surface instabilities such as RTI) just before the bubble minimum, which corresponds with the analysis of Menon and Lal [61] and Shepherd [76]. At this point the RTI develops and, unlike during the initial bubble growth after firing, the surface perturbations are allowed to amplify as the bubble grows. Photographs of the RTI in rebounding vapour bubbles are shown in the work of Shepherd and Sturtevant [77], Frost and Sturtevant [25] and Frost [24]. Pritchett [70] notes the presence of the RTI in a rebounding underwater explosion bubble, and Menon and Lal [61] use a finite-element code to confirm the RTI observed in laboratory underwater explosion experiments.

As the bubble reaches its second maximum (Figure 5.6h), the smaller structures formed by the RTI coalesce and lose their definition, forming a single bubble that then collapses and breaks up into finer bubbles (Figure 5.6i). The RTI that forms at the bubble collapse assists the break up, as some of the protruding glassy bubbles are pinched off by the inward fingers of liquid [76]. As the bubble mass rebounds for the second time, some parts of the main bubble surface give way to smaller glassy bubbles again, though they are generally less evident (Figure 5.6j). During this growth the re-entrant jet closes (Figure 5.6k). The bubble mass continues to pulse and the cycles of coalescence and break up continue as energy is dissipated through the surface instability and turbulence in the surrounding liquid. Ultimately buoyancy becomes dominant and the cloud of bubbles rises to the surface. 


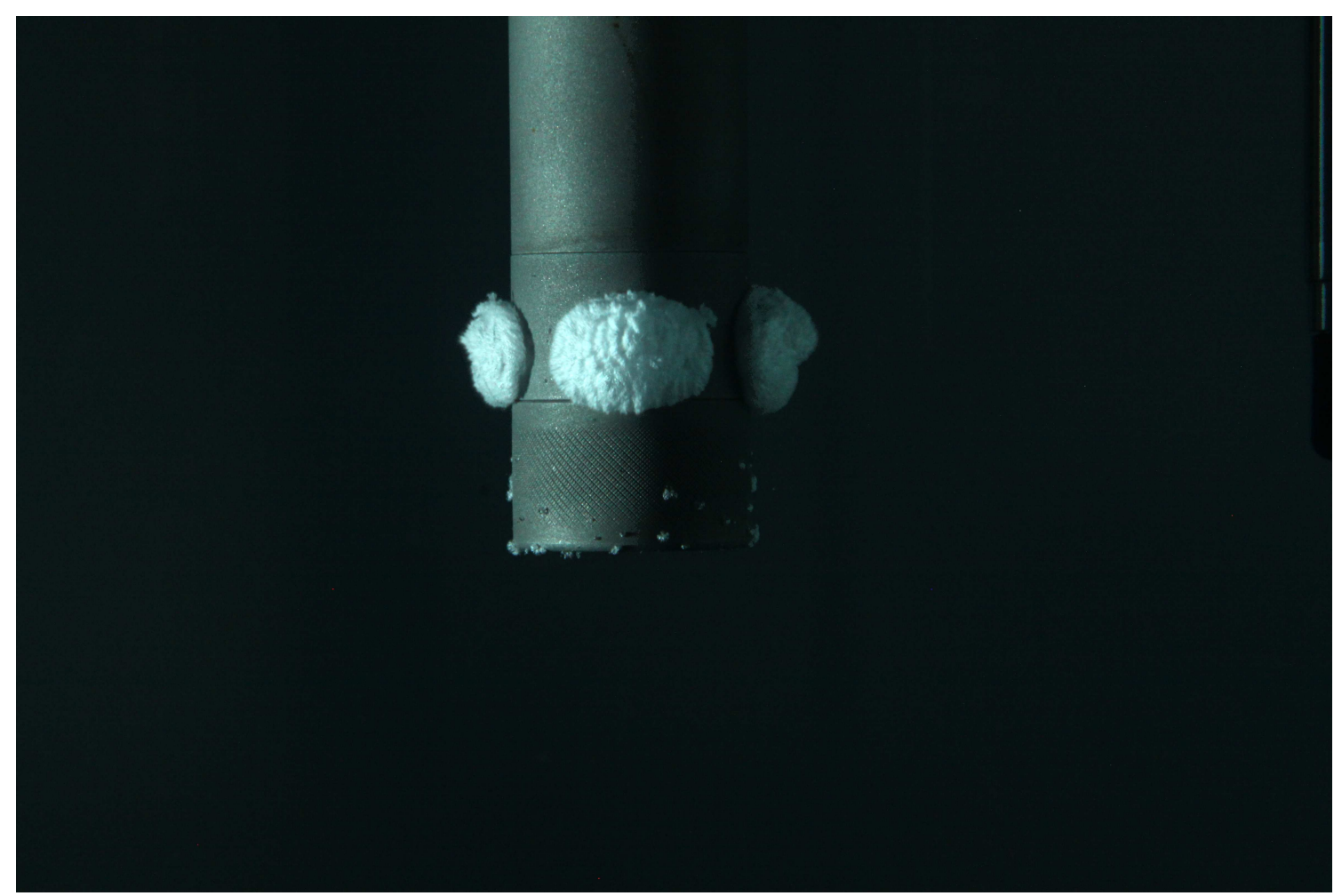

(a) $t^{*}=0.06$. Initial bubble formation just after firing.

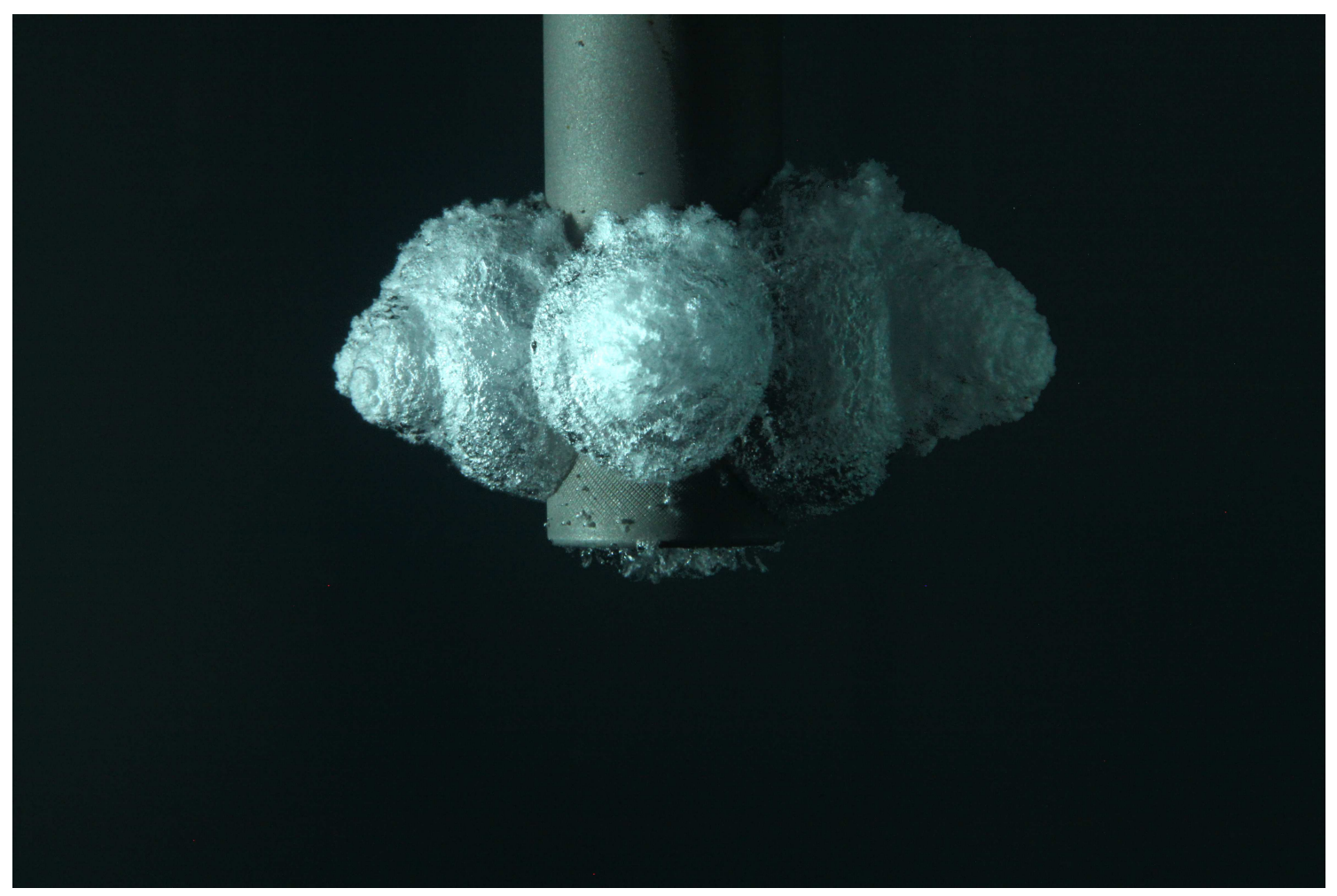

(b) $t^{*}=0.16$. Penetrating air jet forming a second bubble interface.

Figure 5.6: Still images of bubble produced by the scale airgun fired at an initial pressure of 100 bar. A non-dimensional time is given for each image based on the first bubble period. 


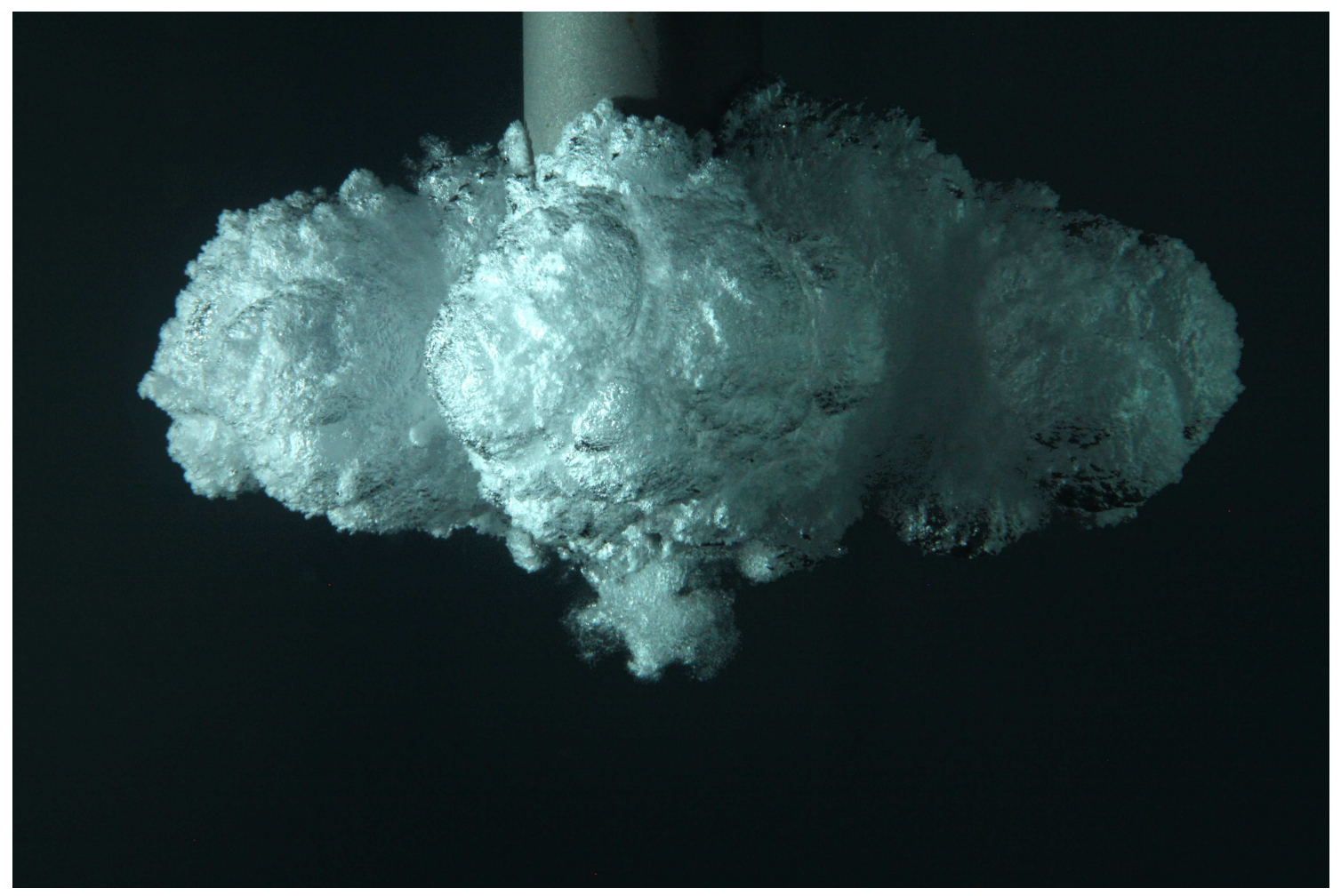

(c) $t^{*}=0.33$. Continued growth of bubble with interfacial perturbations due to jet turbulence.

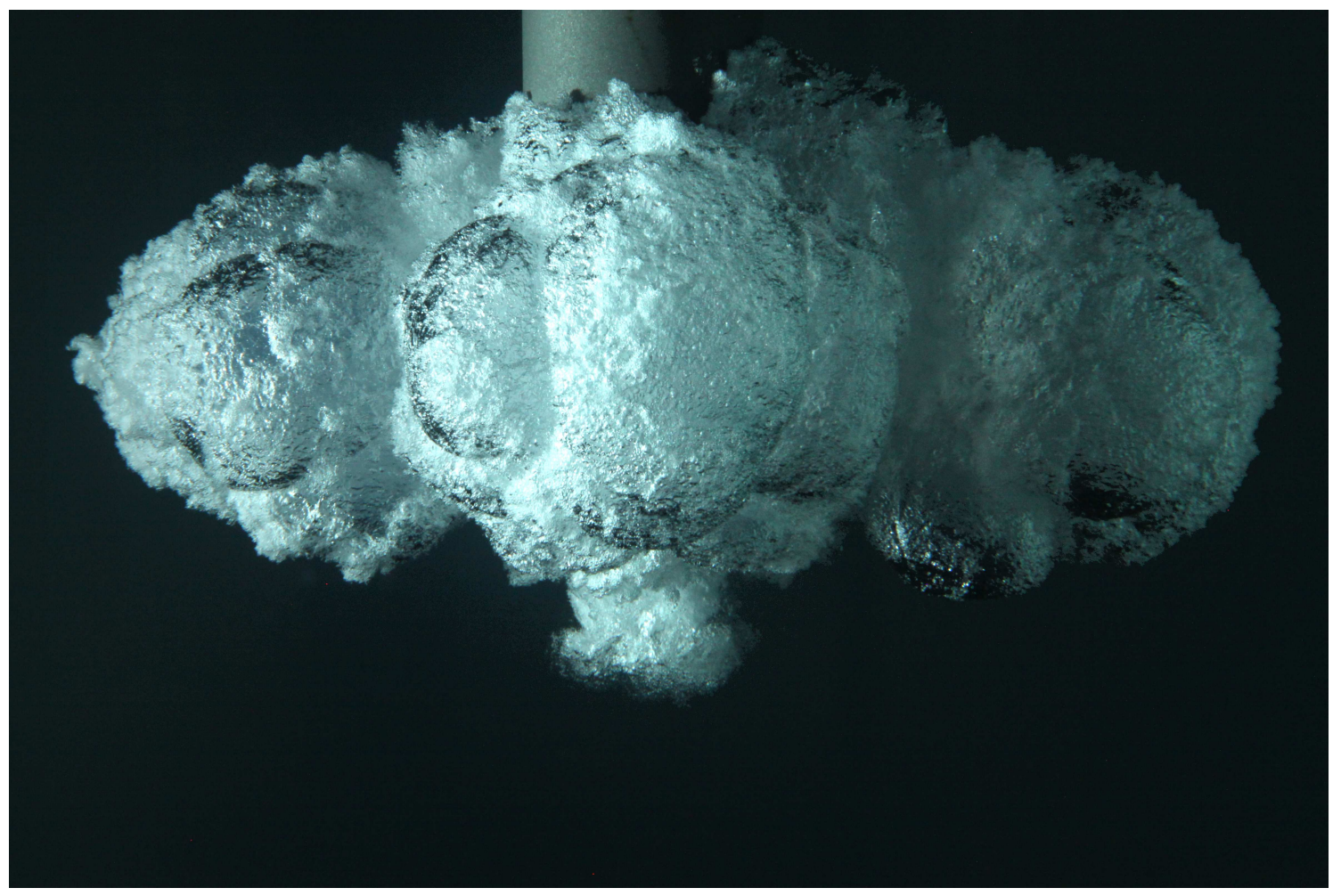

(d) $t^{*}=0.40$. Bubble continues to grow, becoming more glassy, with remnant structure of penetrating jet evident.

Figure 5.6: Continued from previous page. 


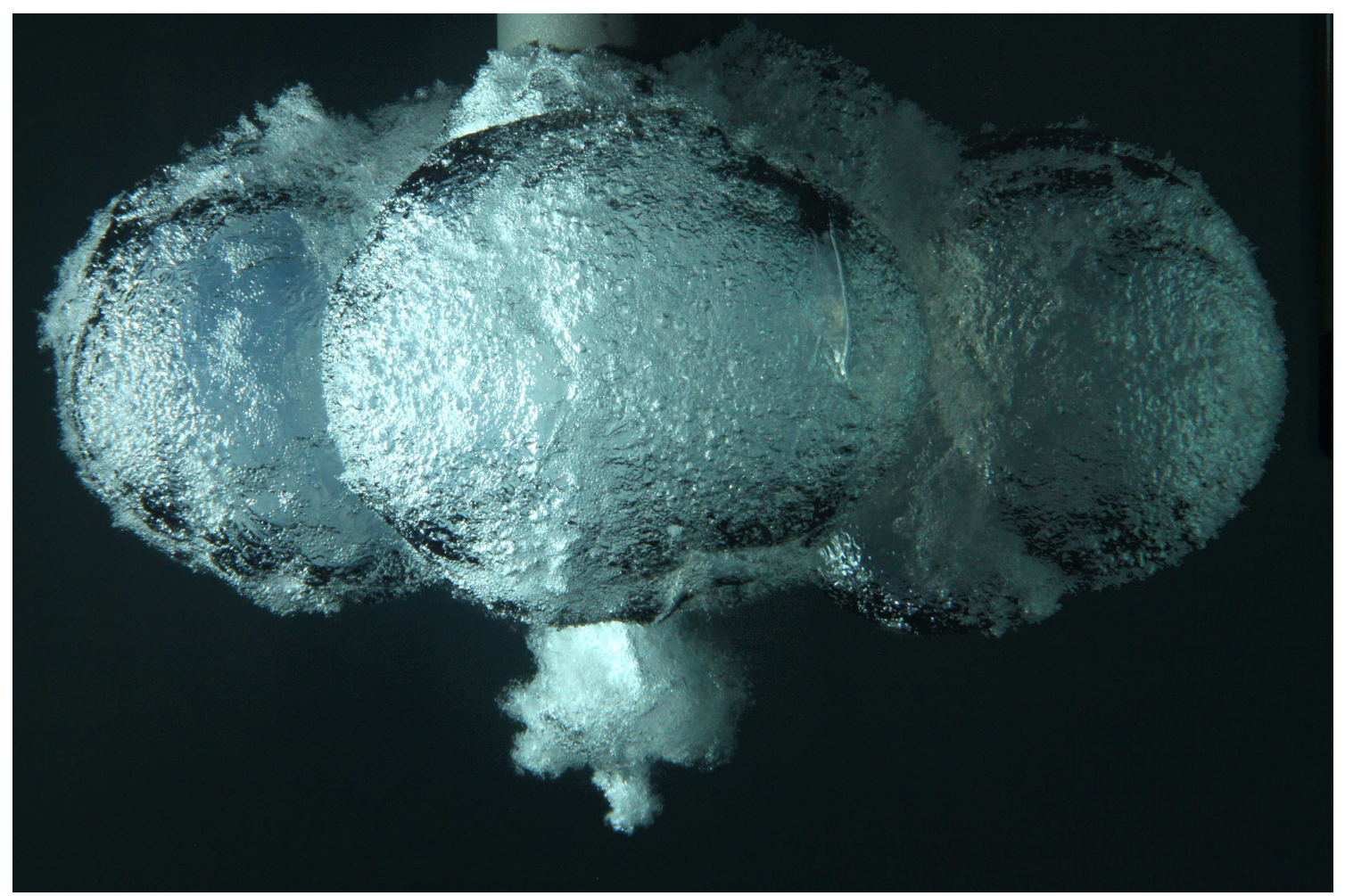

(e) $t^{*}=0.60$. Bubble near maximum with glassy surface. Four distinct bubbles are evident.

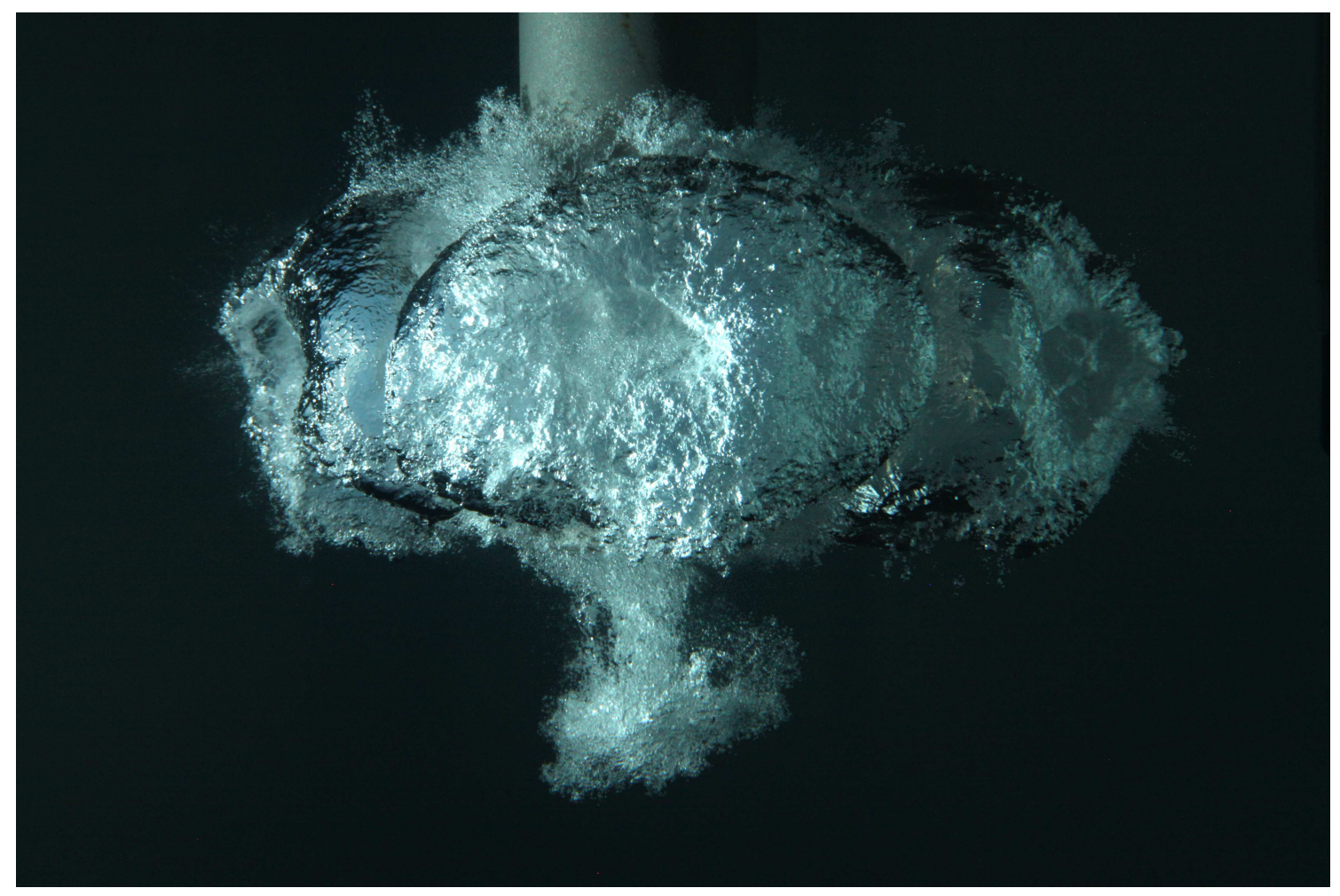

(f) $t^{*}=0.91$. Start of first collapse and formation of re-entrant jet.

Figure 5.6: Continued from previous page. 


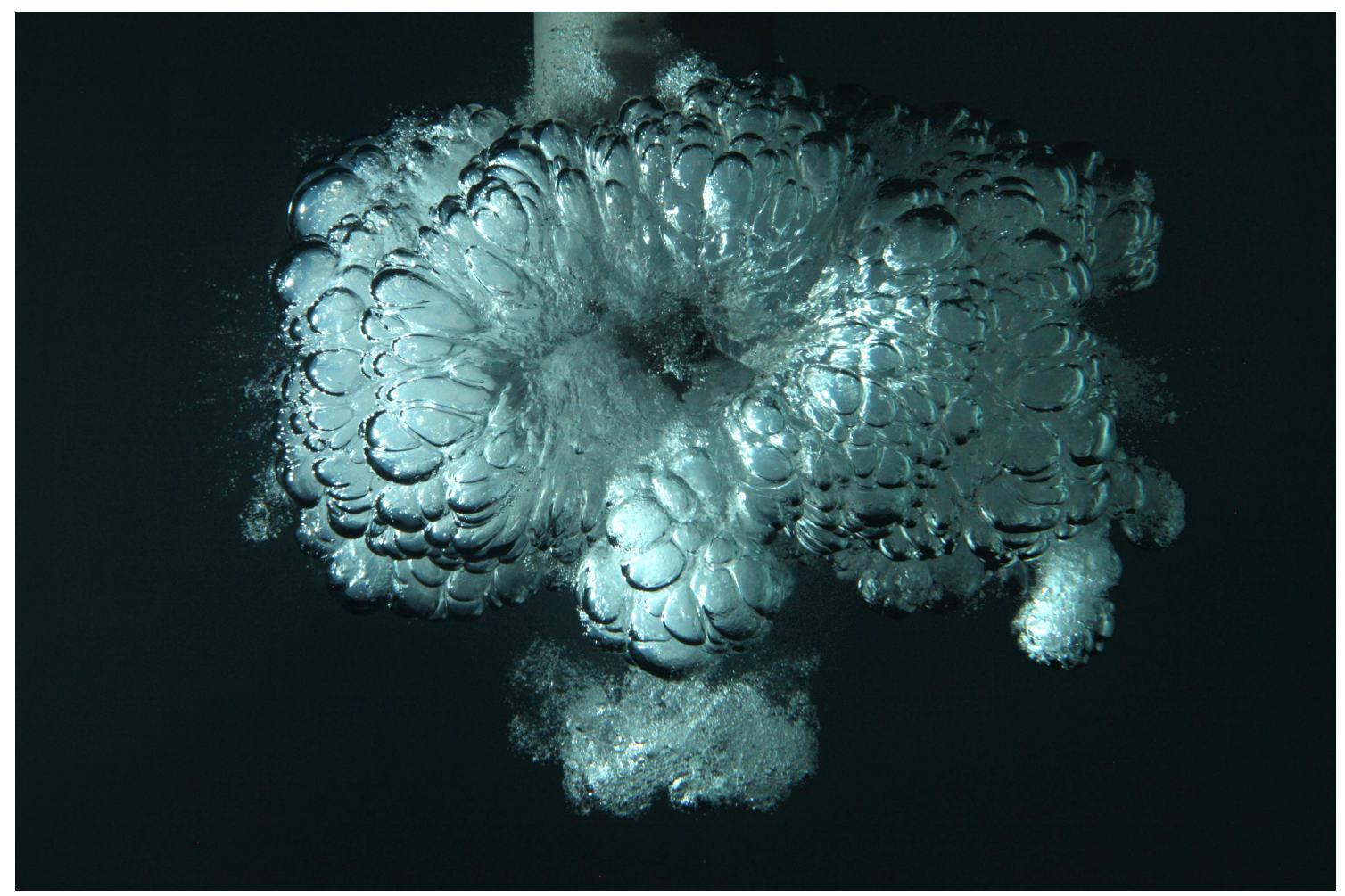

(g) $t^{*}=1.11$. First rebound of bubble (second growth) with re-entrant jet still visible and formation of Rayleigh-Taylor instabilities at interface.

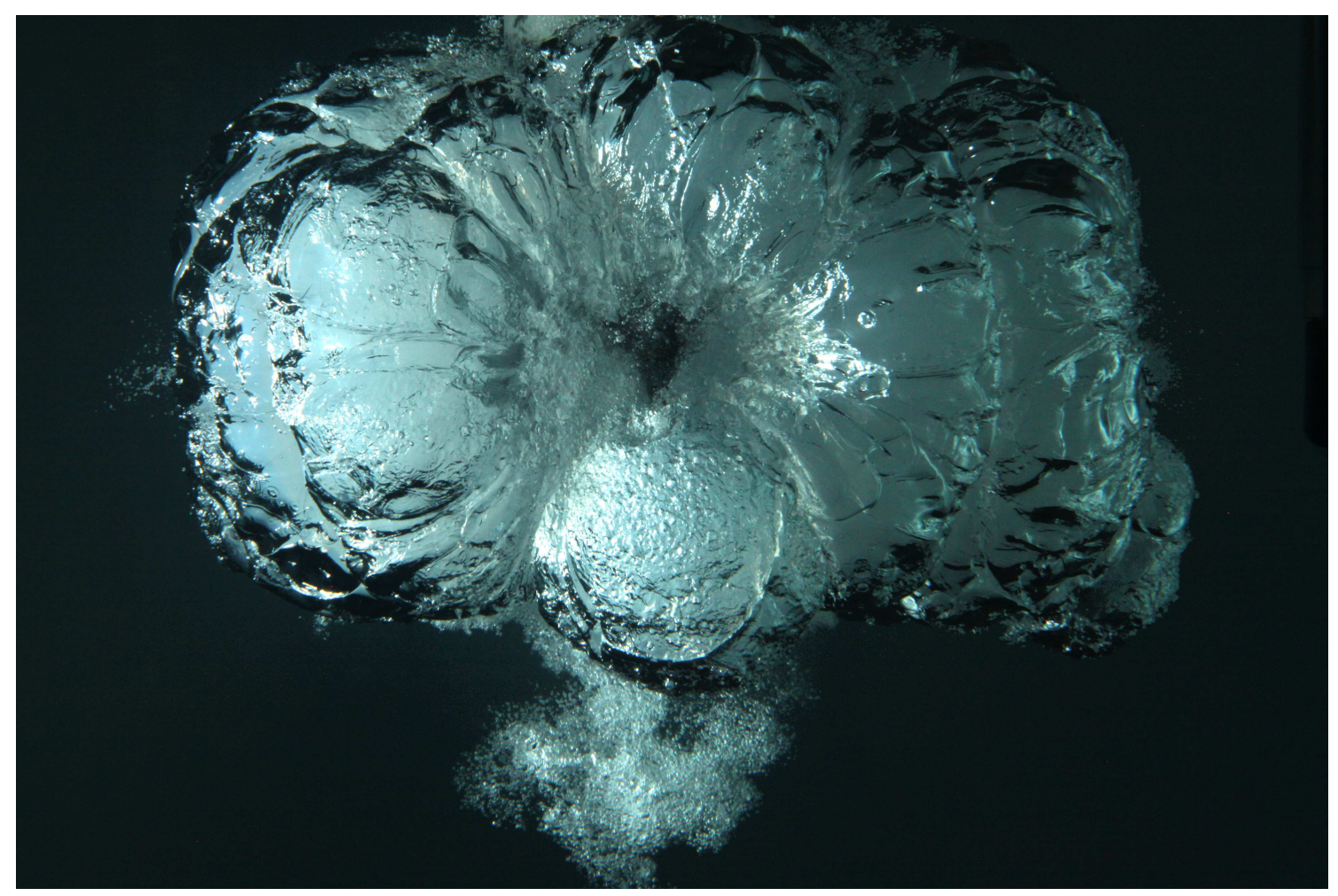

(h) $t^{*}=1.36$. Bubble reaches second maximum, instabilities formed during second growth have grown in volume with the bubble and then lost definition due to deceleration in growth rate.

Figure 5.6: Continued from previous page. 


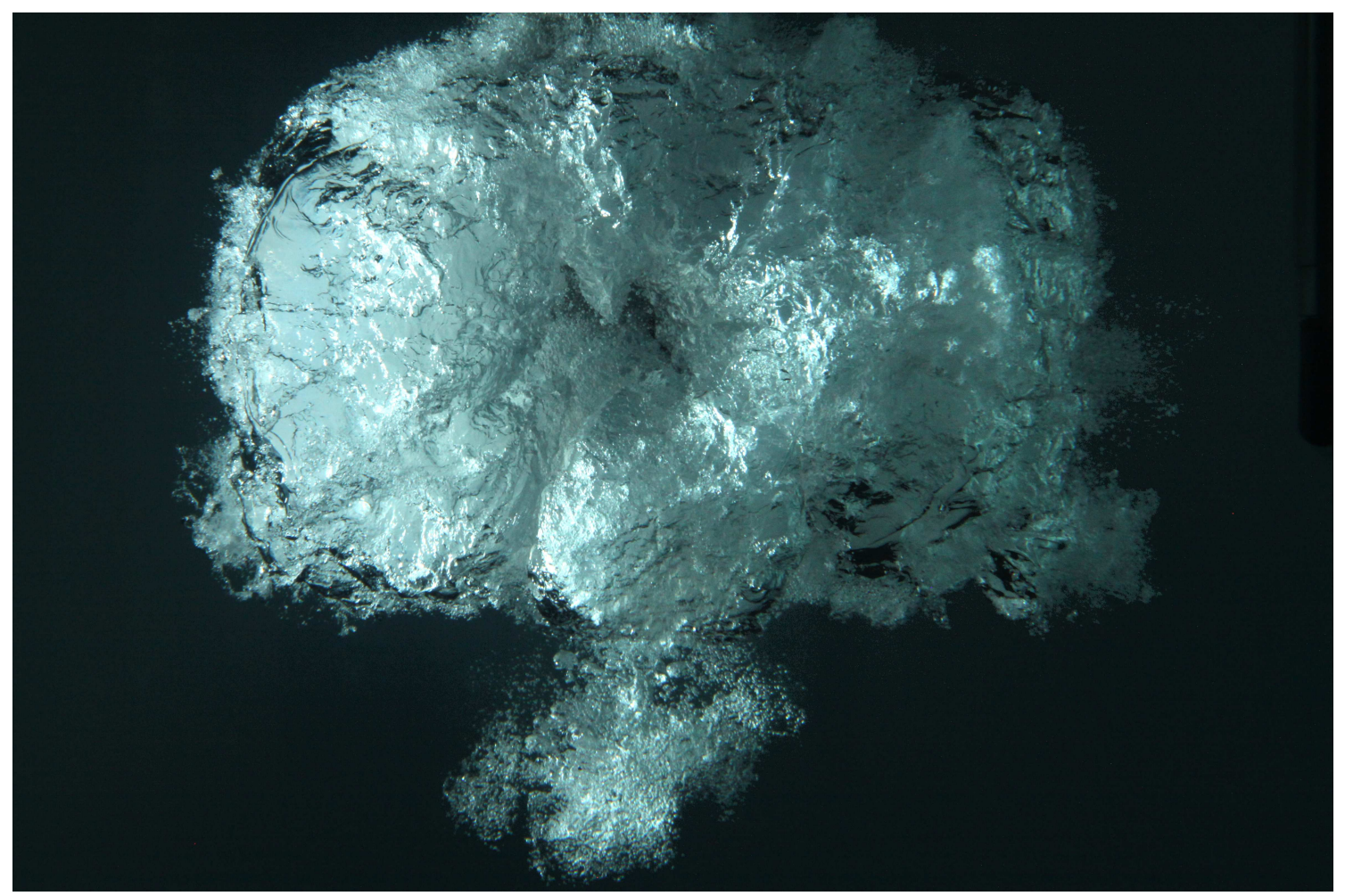

(i) $t^{*}=1.58$. Second collapse.

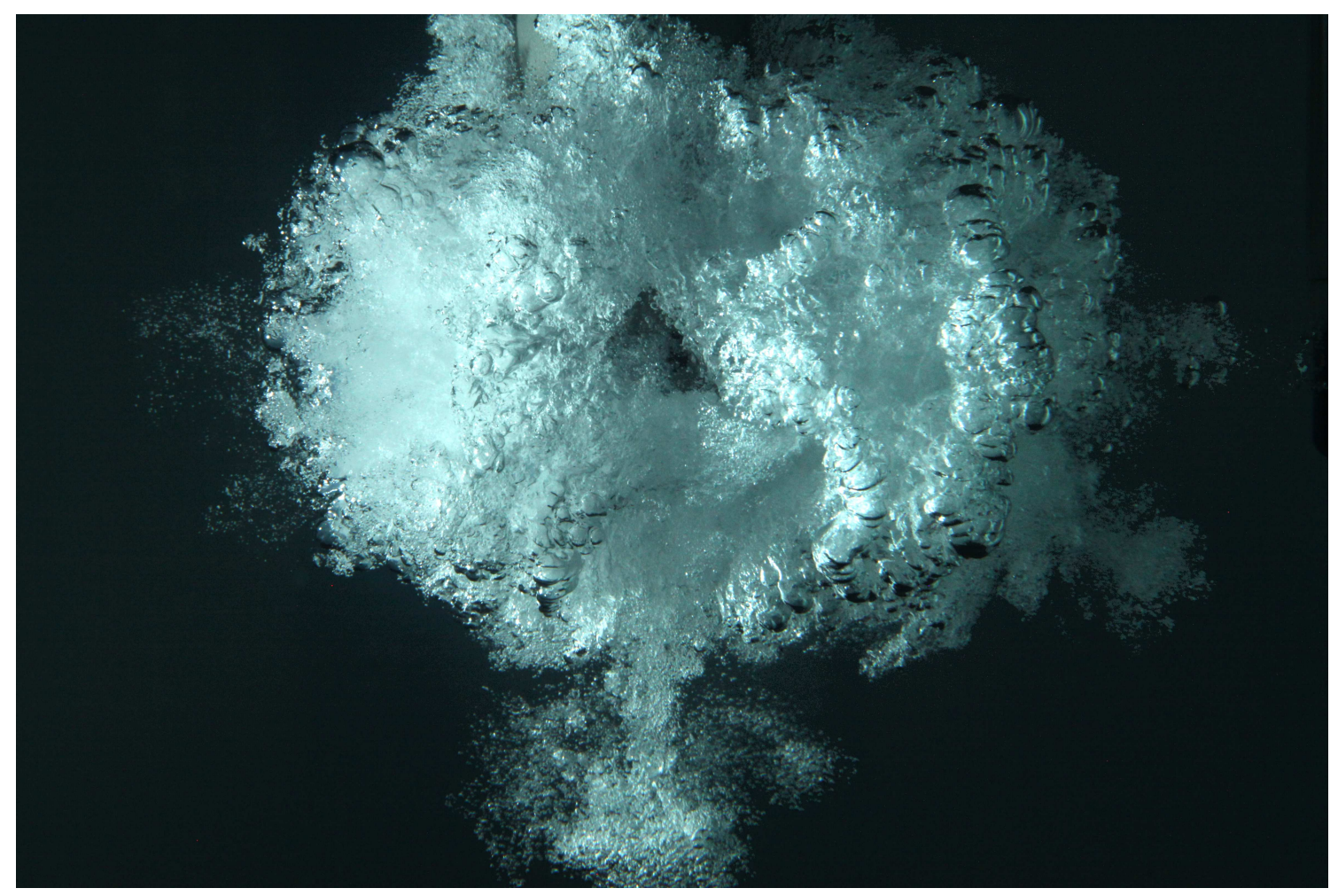

(j) $t^{*}=1.85$. Third growth of the bubble. The hole from the first re-entrant jet is still visible. Rayleigh-Taylor instabilities return, but less evident than before due to turbulent break up.

Figure 5.6: Continued from previous page. 


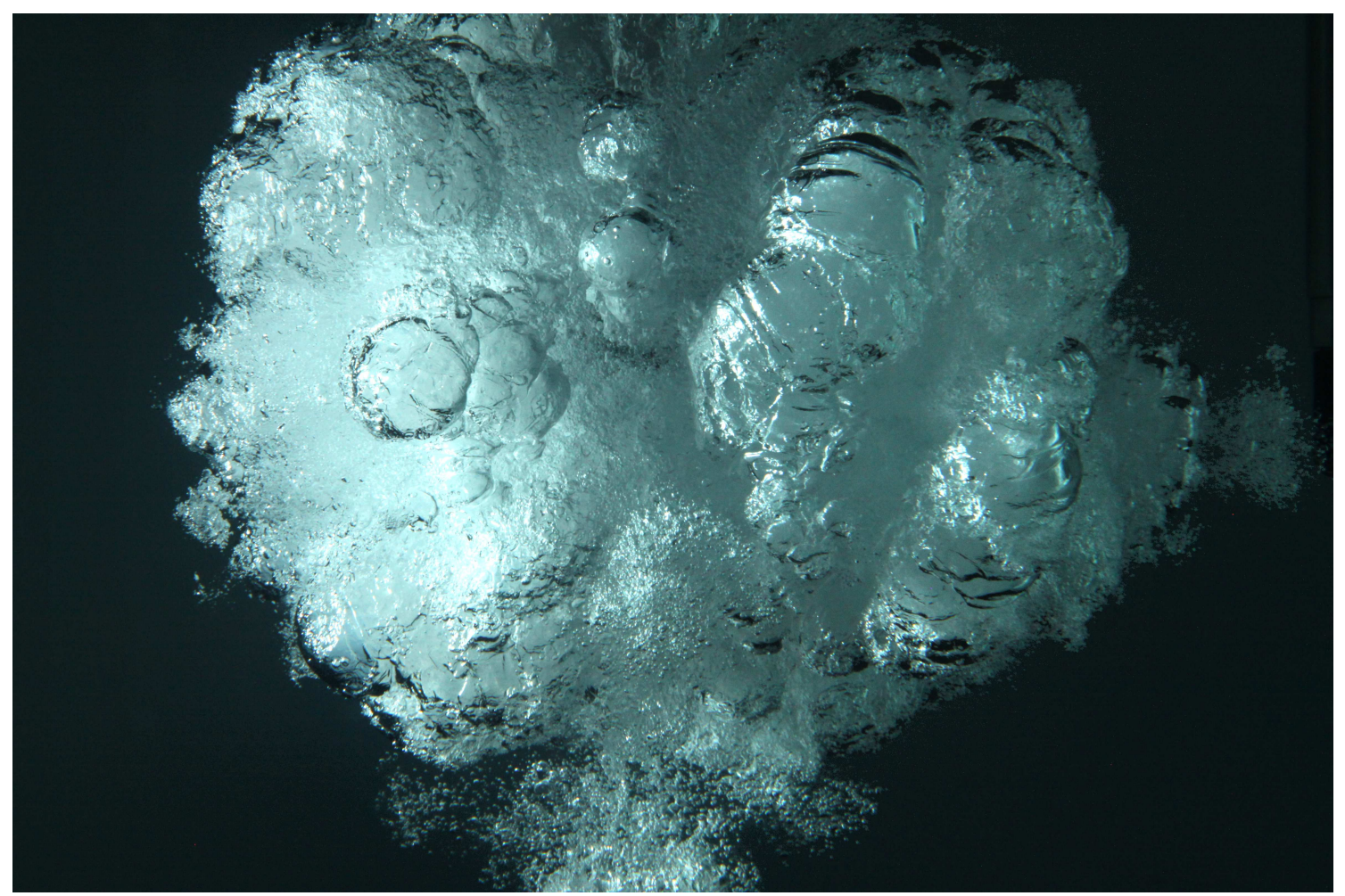

(k) $t^{*}=2.11$. Just before third maximum. The re-entrant jet is no longer visible.

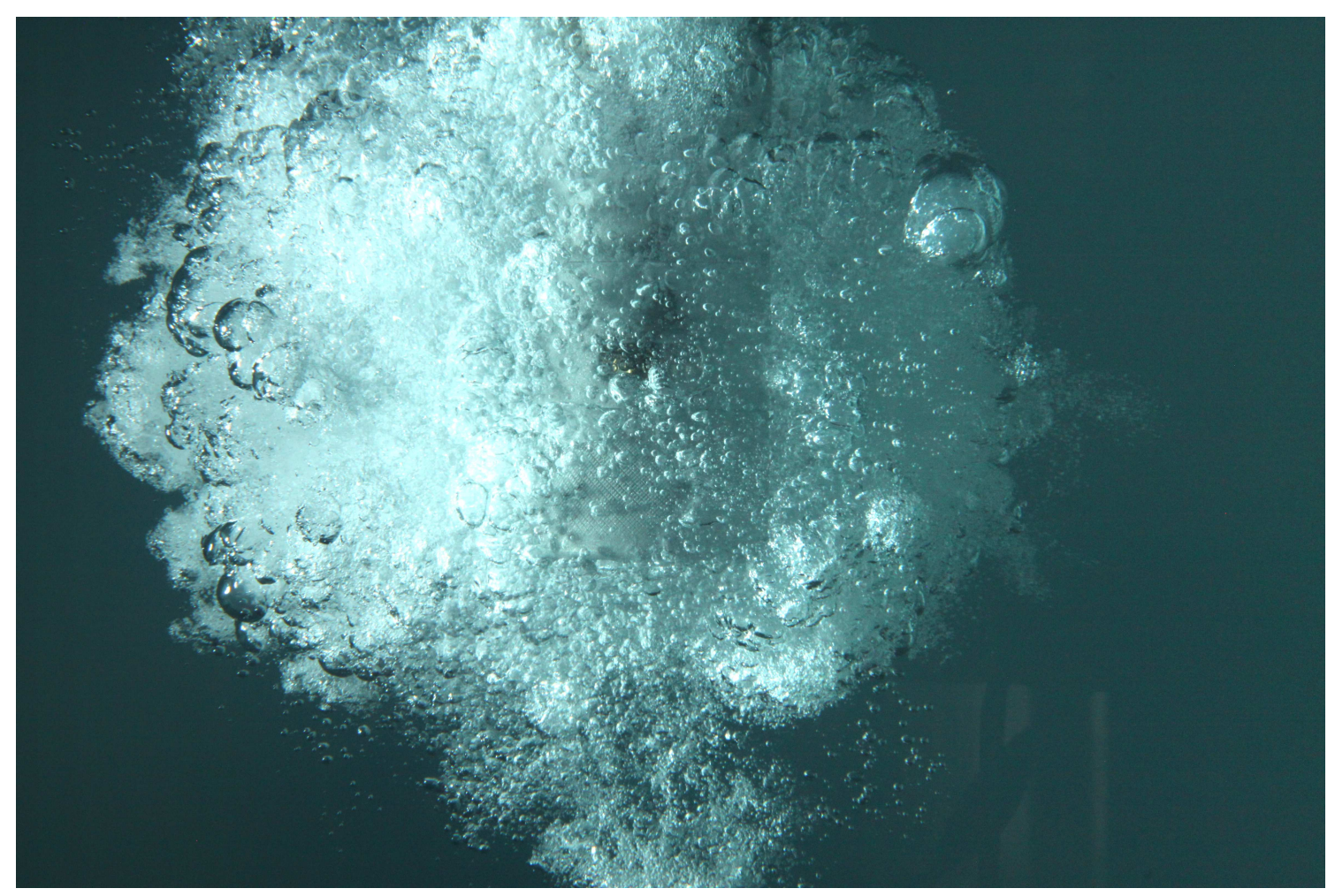

(l) $t^{*}=2.71$. Fourth growth showing break up into a cloud of small bubbles.

Figure 5.6: Continued from previous page. 


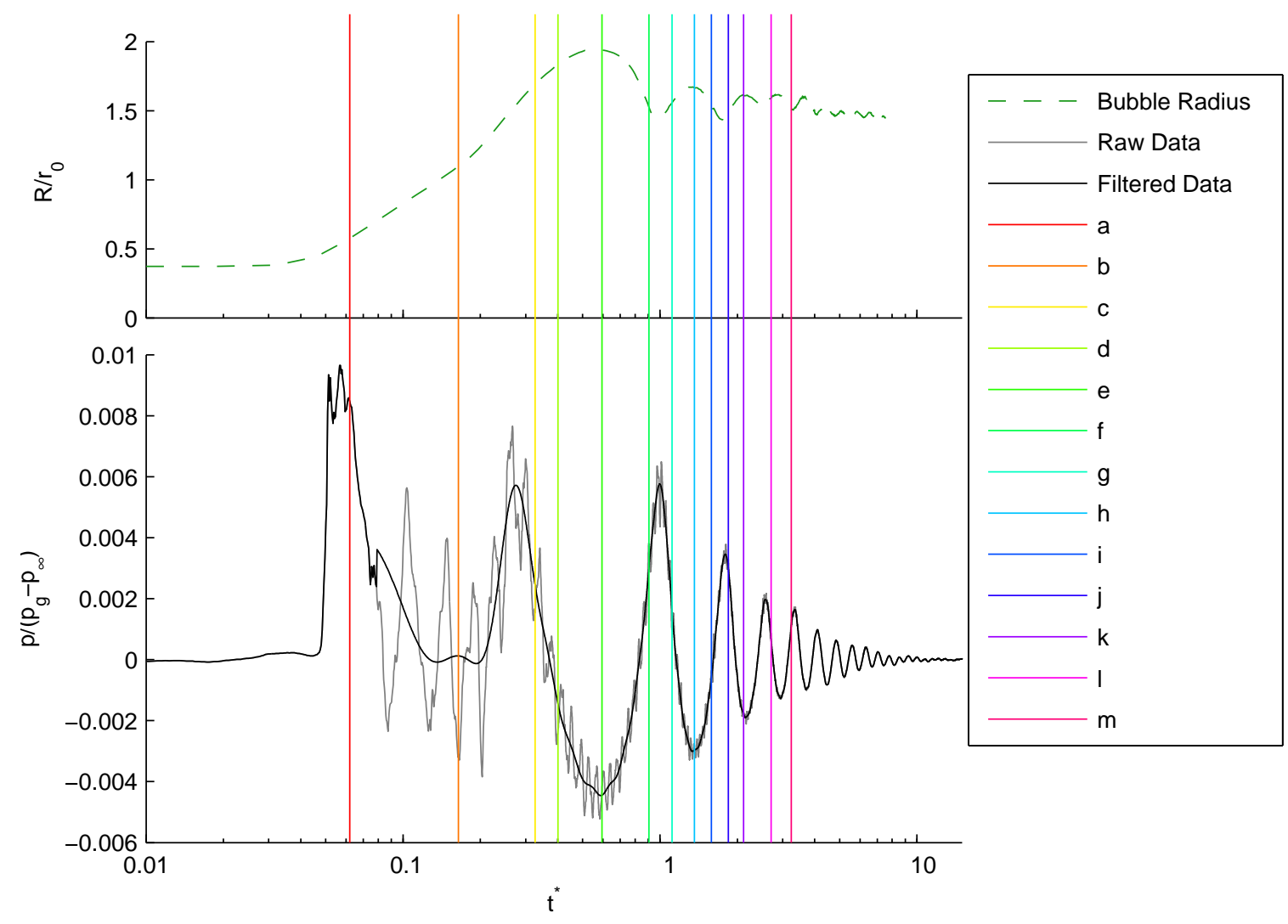

Figure 5.7: Relative point in the bubble's lifetime of images in Figure 5.6. The average radius is calculated from 10 high-speed image sequences and is non-dimensionalised with the equivalent equilibrium bubble radius at ambient pressure. The field pressure response is of one of these 10 firings, aligned accordingly in time and non-dimensionalised with the ambient pressure. Time has been non-dimensionalised with the first bubble period. 


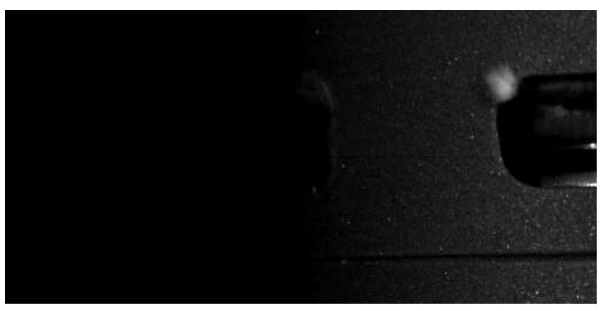

(a) Frame 1

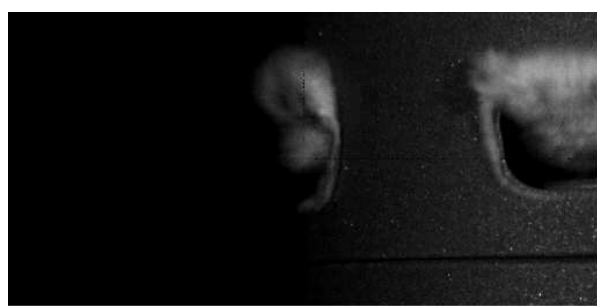

(c) Frame 3

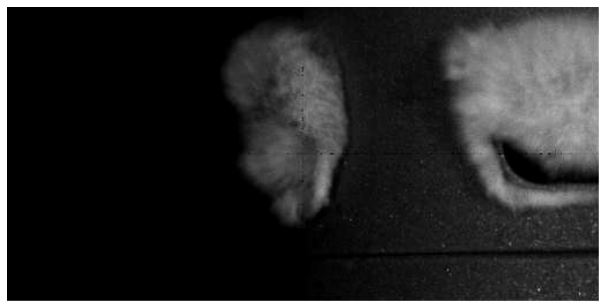

(e) Frame 5

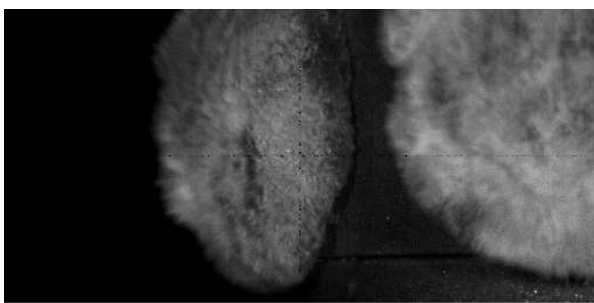

(g) Frame 11

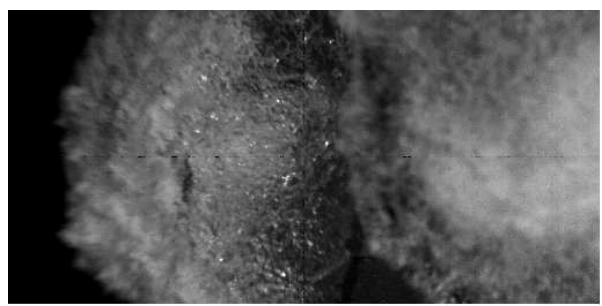

(i) Frame 21

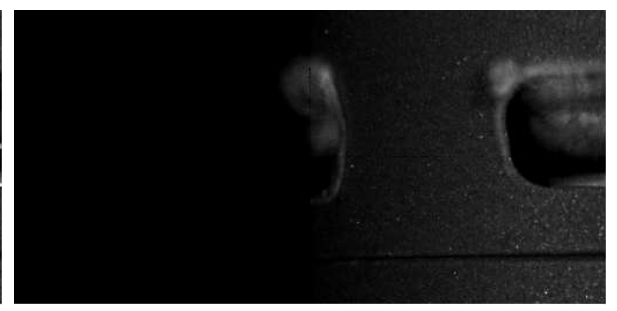

(b) Frame 2

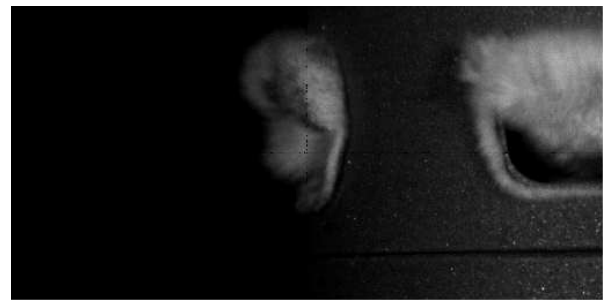

(d) Frame 4

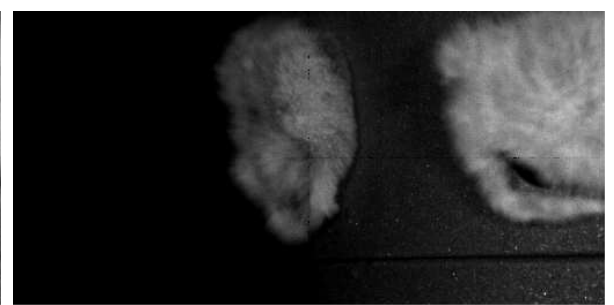

(f) Frame 6

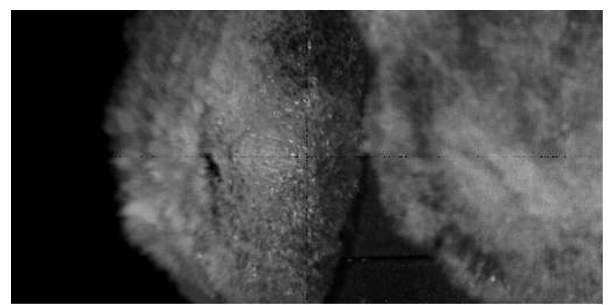

(h) Frame 16

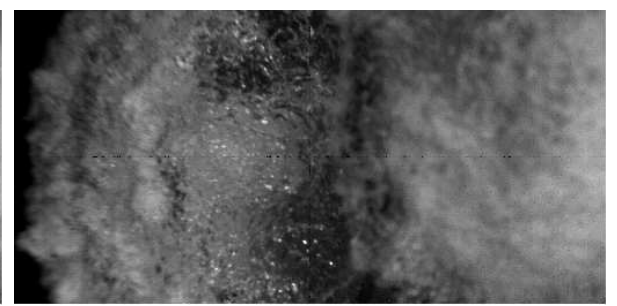

(j) Frame 26

Figure 5.8: Images from $20 \mathrm{kHz}$ high-speed photography (50 $\mu$ s increments) showing initial release of air after firing at 100 bar initial pressure. The cavity around the bottom rim of the port in images $\mathrm{b}-\mathrm{f}$ is potentially cavitation induced by shear stresses or vortex core developed in the liquid. The regular texture of the initial jet (particularly in images $f-j$ ) suggests surface instabilities. 


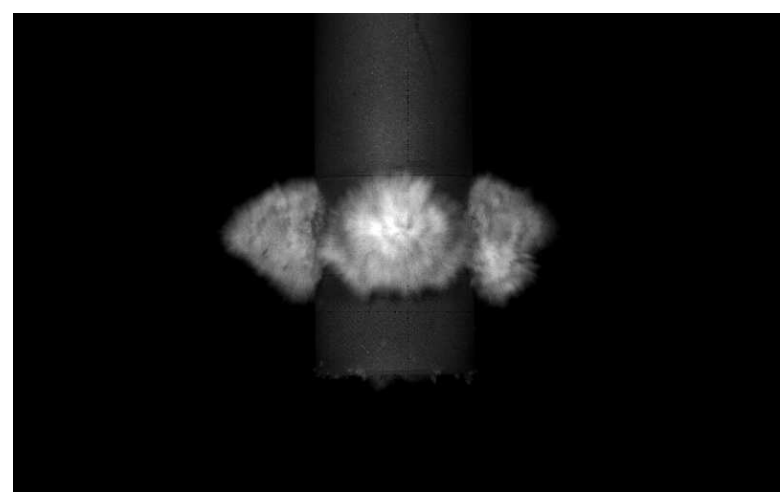

(a)

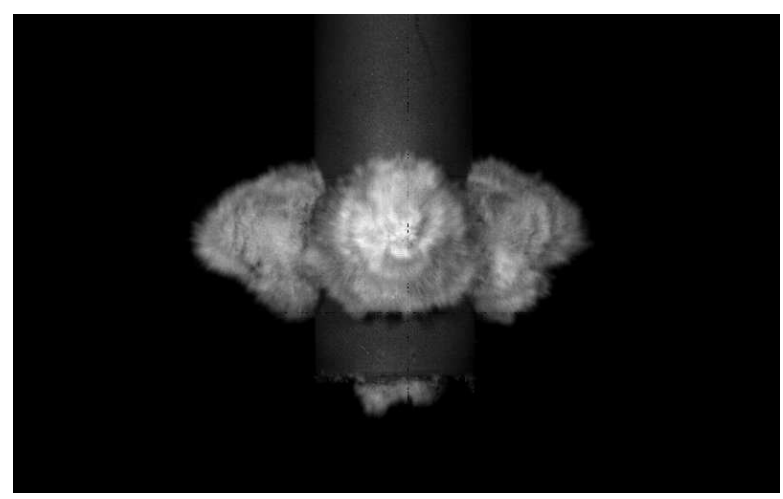

(c)

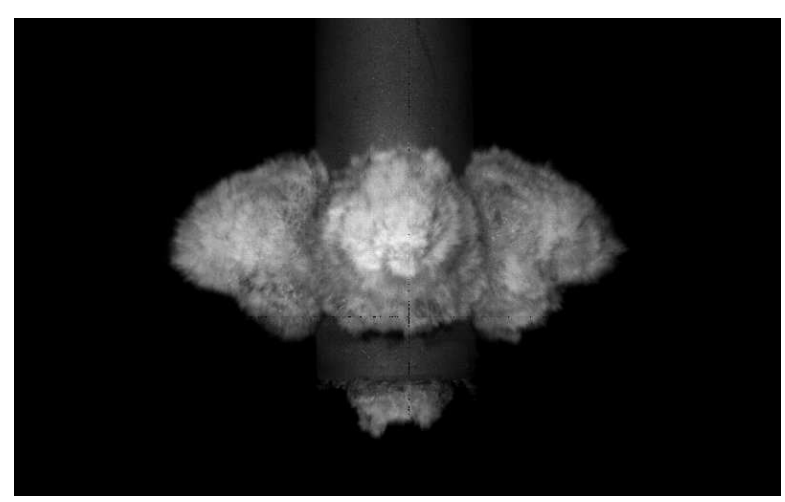

(e)

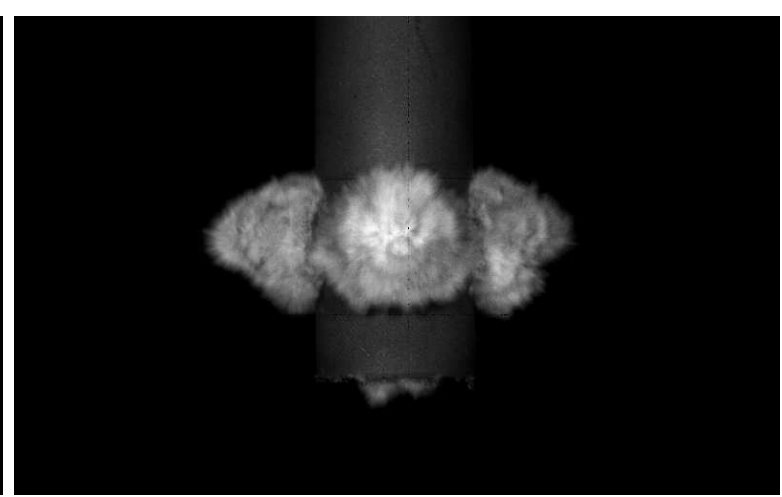

(b)

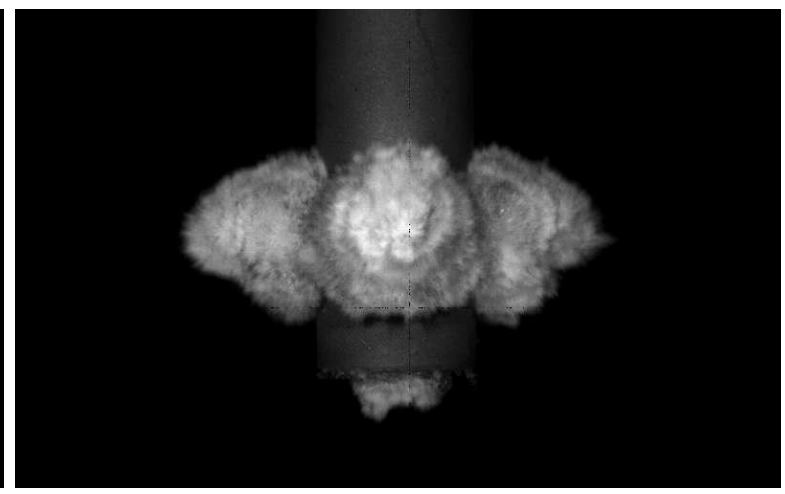

(d)

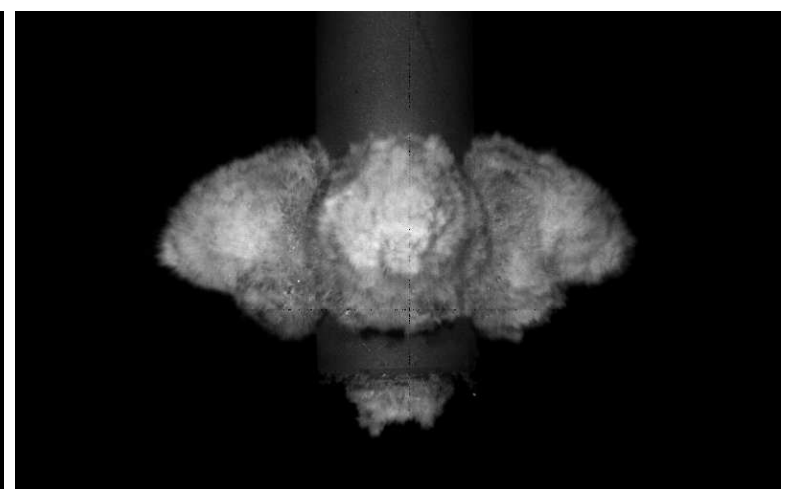

(f)

Figure 5.9: Images from high-speed photography at intervals of $0.33 \mathrm{~ms}$ showing jet of air penetrating initial growing bubble interface for 100 bar initial airgun pressure. Images a - c show pointed form of penetrating jet. By image $d$ the difference in surface texture between the slightly glassier bubble (close to airgun body) and faster moving jet is becoming visible. Images are at non-dimensional times with respect to the first bubble period of $t^{*}$ from 0.04 to 0.21 . 


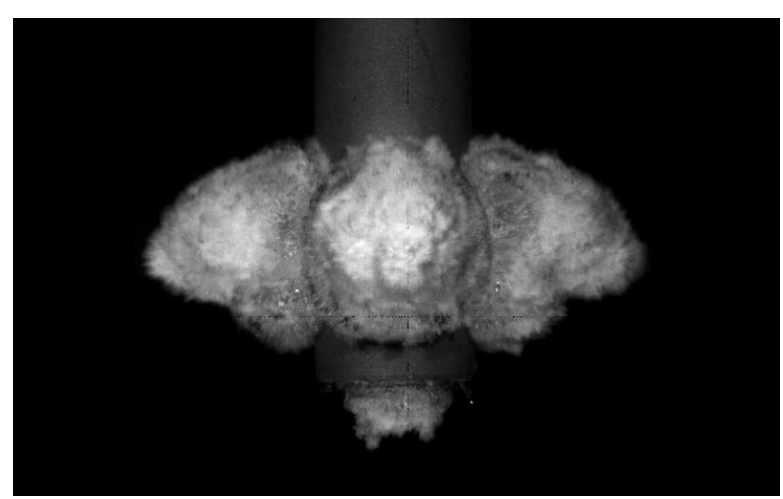

(g)

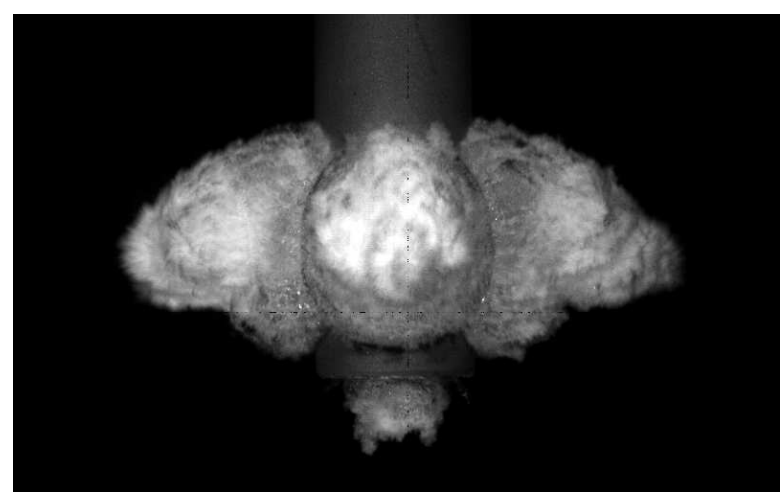

(i)

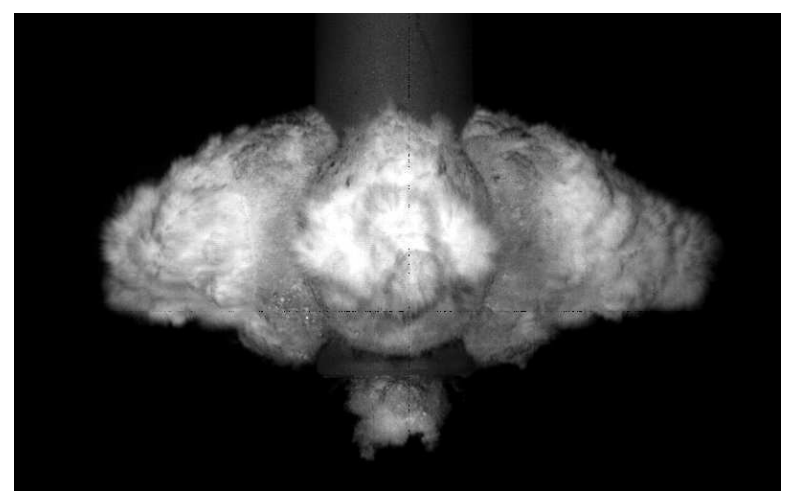

(k)

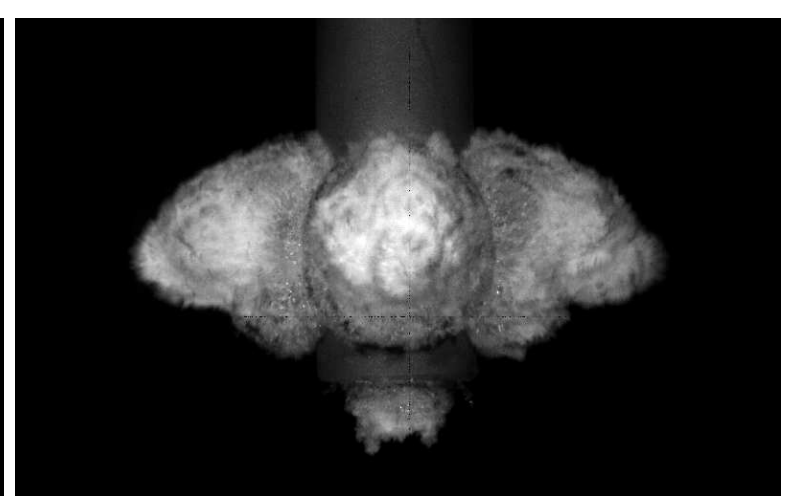

(h)

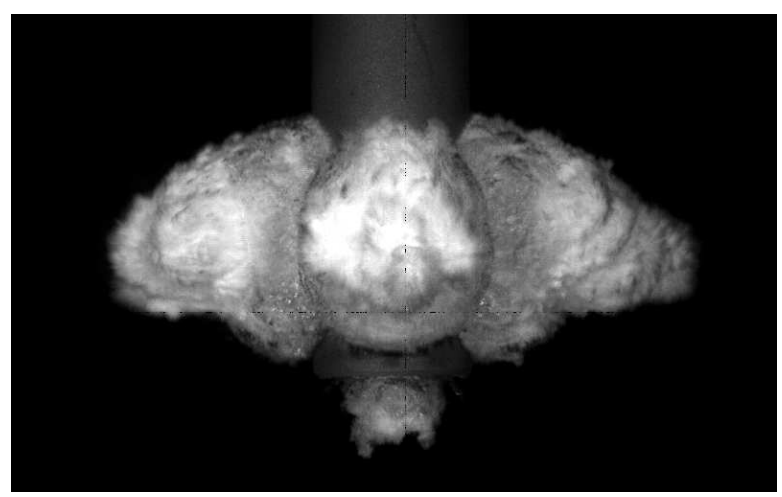

(j)

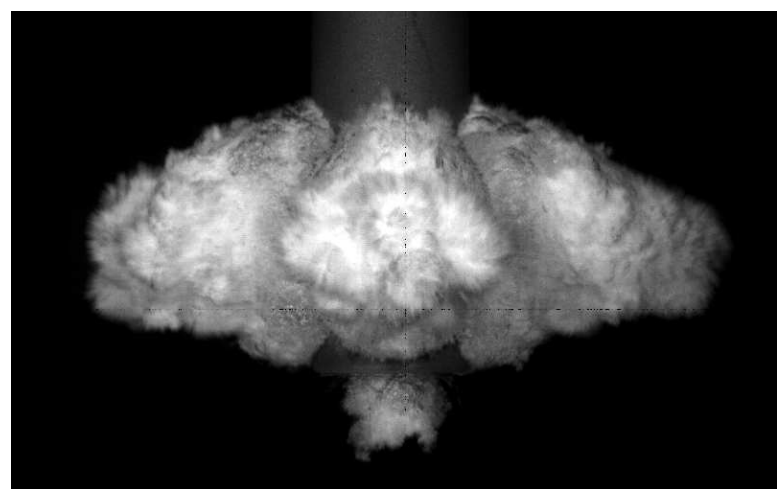

(l)

Figure 5.9: Continued from previous page. 


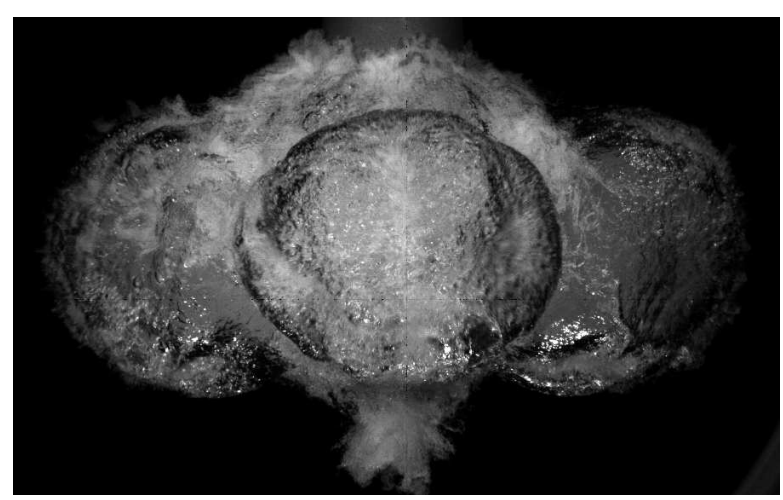

(a)

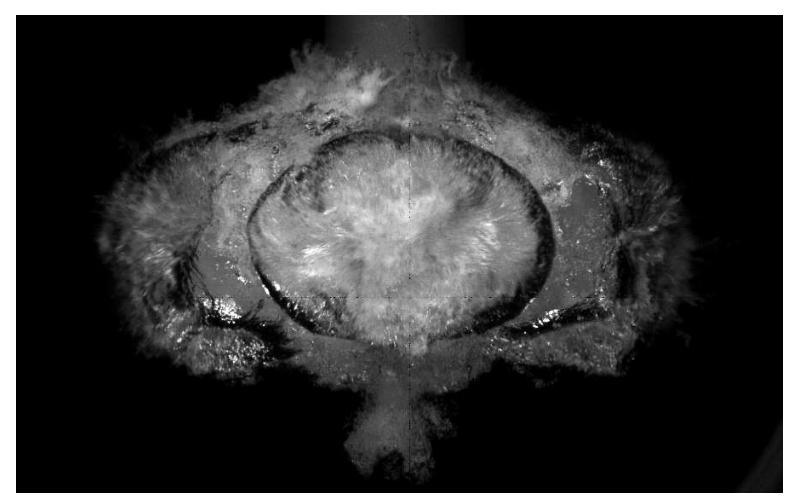

(c)

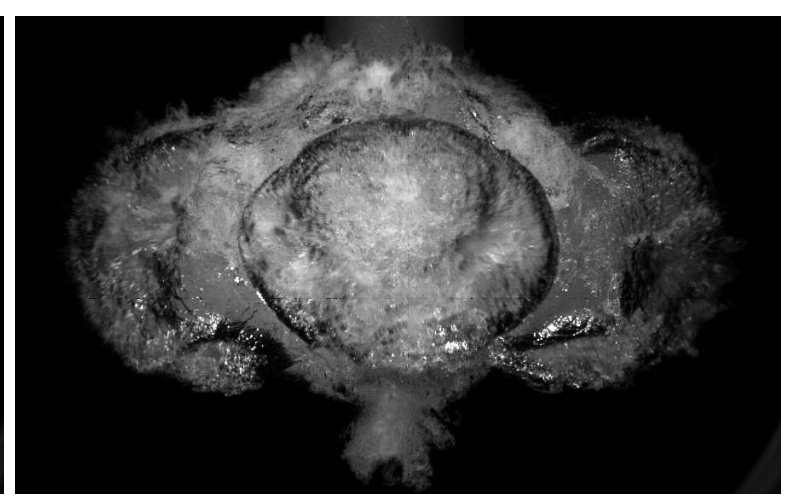

(b)

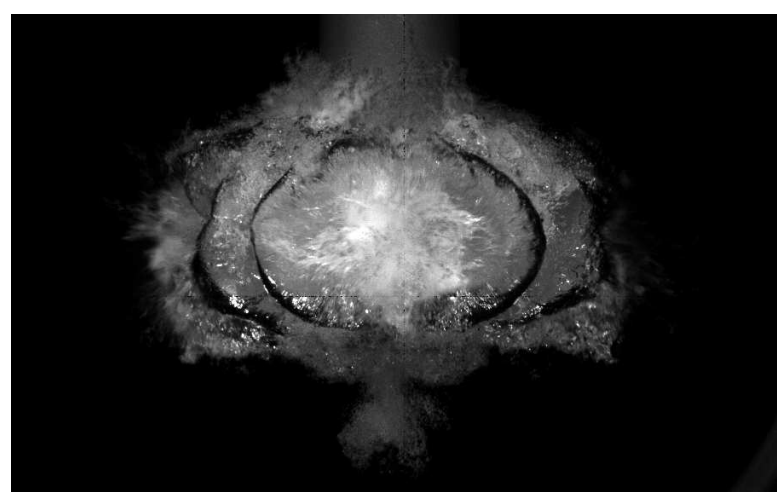

(d)

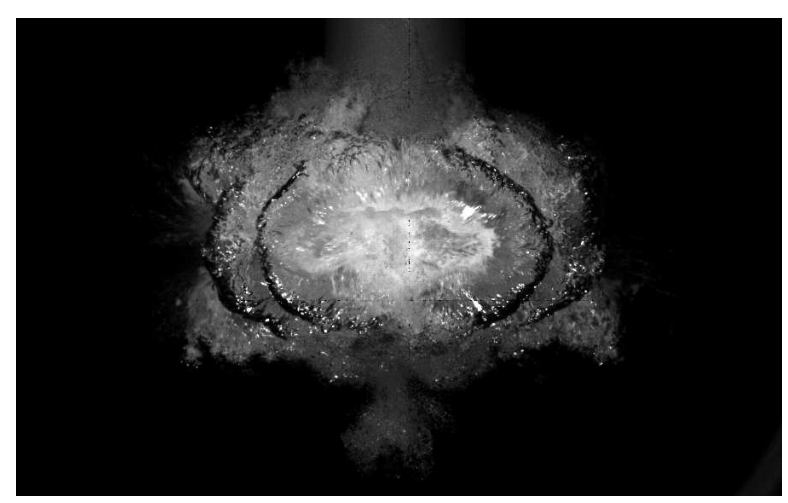

(e)

Figure 5.10: Images from high-speed photography at intervals of $1.67 \mathrm{~ms}$ showing formation of re-entrant jet during first bubble collapse for 100 bar initial airgun pressure. Images are at non-dimensional times with respect to the first bubble period of $t^{*}$ from 0.68 to 0.98 . 


\subsubsection{Bubble Dynamics Near Boundaries}

The airgun was fired at an initial pressure of 100 bar at several standoff distances from the steel wall and the free surface to investigate the effect of a boundary on the bubble dynamics. To assess the interaction with the free surface, the depth of the water was reduced incrementally with the airgun fixed in the centre of the tank. The fixed position of the airgun allowed photography to be taken through the viewing window, and the wall pressure response to be measured at the same depth as the bubble.

As noted in [17] (Chapter 4) the frequency of the bubble oscillations increased as the free surface standoff distance was reduced. The airgun was fired at standoff distances of 5.46, 3.32, 1.15 and 0.75 bubble radii (maximum radius measured in z-direction). Figure 5.11 shows that at an initial pressure of 100 bar, there is evidence of the bubble interaction with the surface at the two smallest standoff distances (1.15 and 0.75). A vertical split forms at the top of the bubble as the re-entrant jet forms towards the airgun body and away from the free surface. The split forms during the first bubble collapse and does not begin to close until the second maximum. As expected, the partly downward direction of the re-entrant jet moves the bubble away from the surface slightly. These two smaller standoff distances are also those for which a significant change in bubble period was found, confirming interaction between the surface and the bubble. Figure 5.11 can be compared with Figure 5.6 (specifically images $\mathrm{f}-\mathrm{h}$ ) and Figure 5.10, which show cases where no boundary interaction is present. Chahine [8] found the limiting distance for interaction of spark-generated bubbles with the free surface to be 4 bubble radii. The smaller limit found here is likely to be affected by the presence of the airgun body. The airgun was also fired at various standoff distances, between 4.51 (airgun at the centre of the tank) and 1.50 bubble radii, from the steel wall (maximum radius measured in the $\mathrm{x}-\mathrm{y}$ plane for 100 bar initial pressure). The smallest standoff distance was limited by the configuration of the tank frame. Due to the tank wall and frame it was difficult to angle the camera perpendicularly to the bubble; however, there was no evidence of any notable change in the dynamics of the bubble. As the bubble period, which can be found from the field pressure response, also remained unaffected, there appears to be no interaction of the airgun bubble with the solid boundary for these cases. Shima [78] found the limiting distance for interaction of spark-generated bubbles with a solid boundary to be 3 bubble radii, with no re-entrant jets forming until the bubble was within 1.5 bubble radii. The presence of the airgun body substantially changes the flow dynamics around the bubble that are the cause of bubble migration towards and jetting onto nearby solid boundaries. As already shown, the bubble jets towards the airgun body, and evidently the presence of an external solid boundary is not enough to significantly change this 


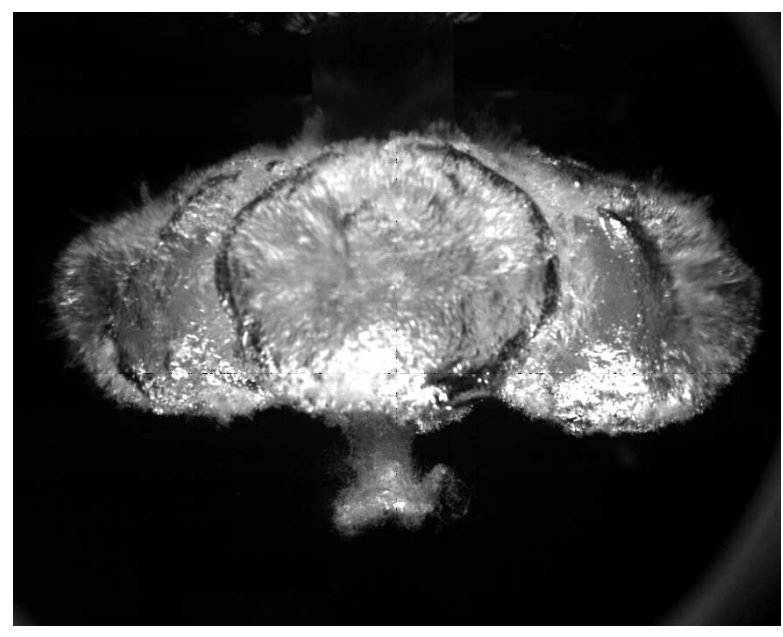

(a)

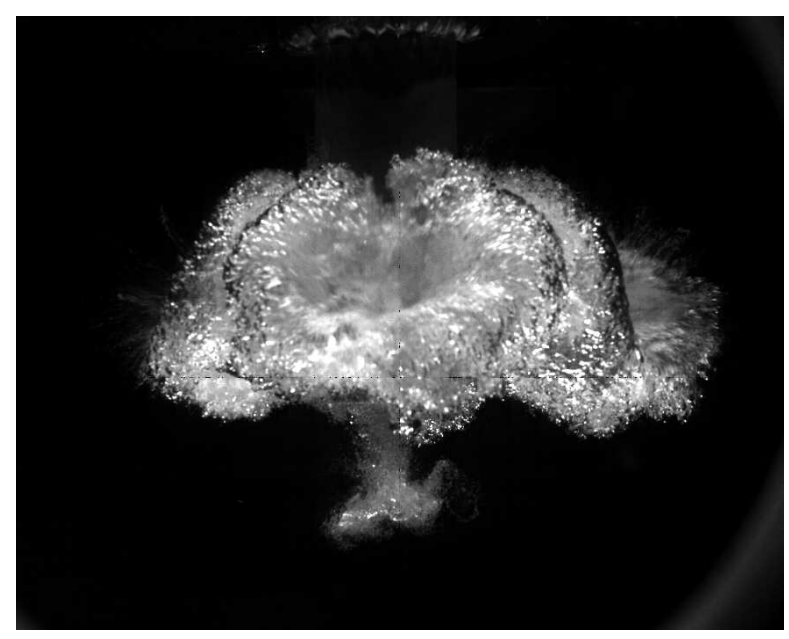

(c)

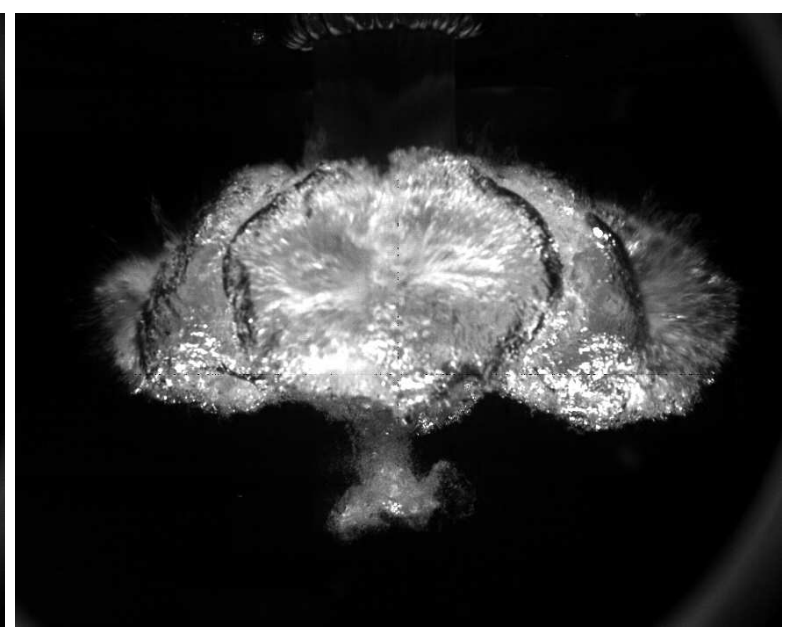

(b)

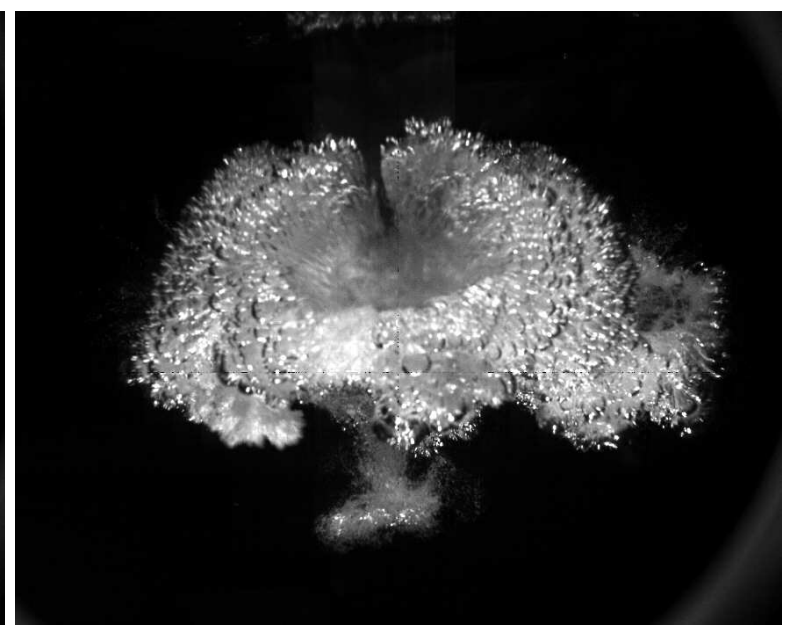

(d)

Figure 5.11: Images from high-speed photography at intervals of $1.67 \mathrm{~ms}$ showing formation of re-entrant jet during first bubble collapse and subsequent second bubble growth at a standoff distance of 0.75 bubble radii from the free surface for 100 bar initial airgun pressure. The reflection of the bubble on the surface of the water can just be seen at the top of the frame particularly in images a $-d$. Images are at non-dimensional times with respect to the first bubble period of $t^{*}$ from 0.81 to 1.39 . 


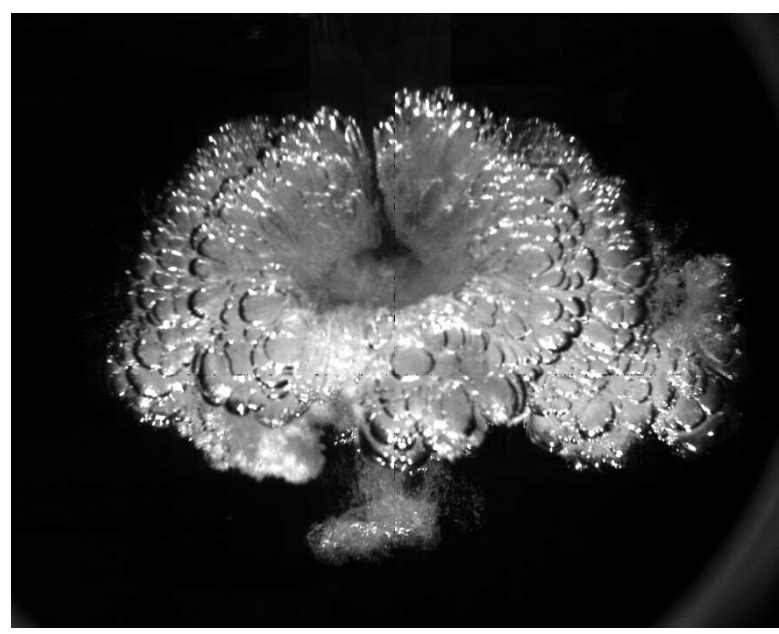

(e)

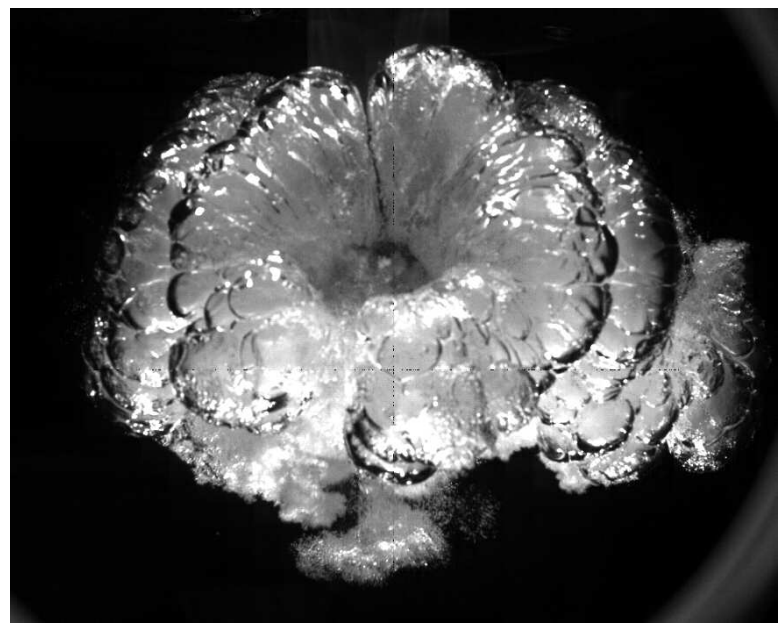

(g)

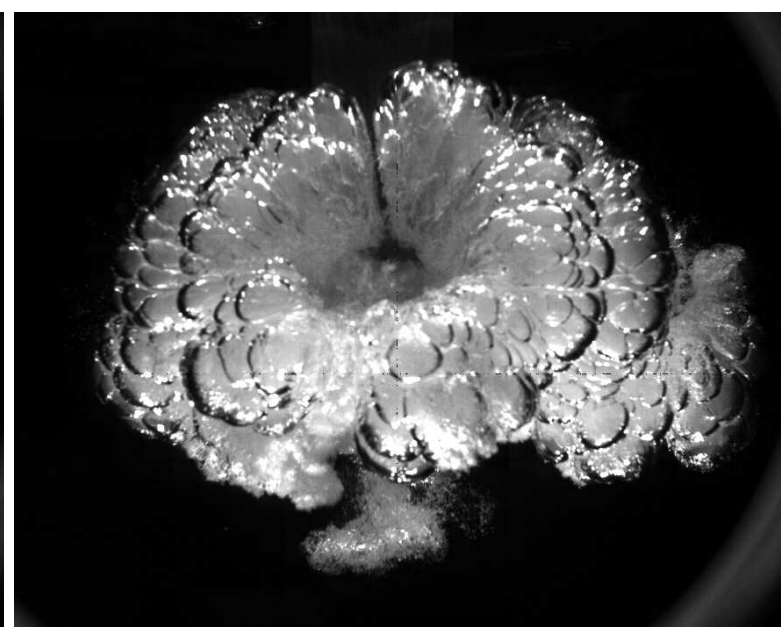

(f)

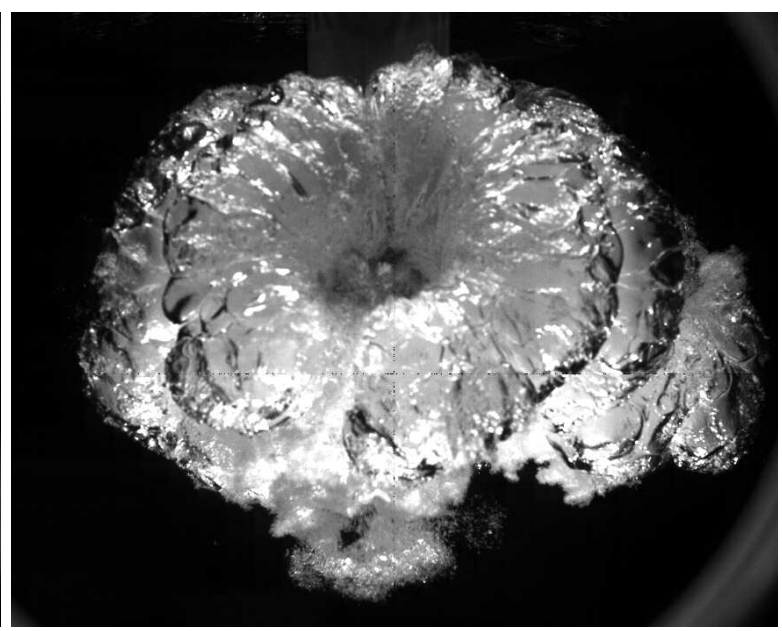

(h)

Figure 5.11: Continued from previous page. 
process. This may change if the airgun were to be fired within 1 bubble radius of the wall.

The effect of the orientation of the model-scale airgun on the bubble's tendency to interact with a nearby surface is unquantified; however, it should be taken into consideration that bubbles form re-entrant jets directed away from a free surface (which would be in the direction of the airgun body, onto which the airgun bubble already jets) and towards a solid boundary (in the opposite direction to which the airgun bubble jets); therefore, orientation may be relatively inconsequential. The fact that the first bubble maximum is actually four distinct bubbles also impacts the hydrodynamic flow and pressure field, making the bubble's behaviour for a closer standoff distance difficult to comment on without experimental data.

\subsubsection{Bubble Radial Growth, Velocity and Acceleration with Field Pressure}

Shadowgraph photography of the bubble produced by the airgun fired at 50 and 100 bar initial pressures was filmed at $3 \mathrm{kHz}$ and has been analysed to determine the radius, velocity and acceleration of the bubble surface. For each image sequence, the interface was tracked and the maximum bubble radius was found in each frame. The bubble radius is taken as the horizontal distance from the central vertical axis of the airgun to the edge of the bubble at its widest point, ie: the bubble is considered globally as one volume because it oscillates as one body, despite the four individual bubbles evident at the first maximum (and after this point, the air volume is no longer separated). This data was then smoothed with a moving 4 point quadratic and the average of 10 sets of data for each initial pressure was used to determine the overall radial growth. The velocity of both averaged bubbles was found using a central difference method, filtered with a moving 10 point quadratic, and the mid-point gradient was used to obtain the acceleration. In order to capture the initial phenomena in greater detail, $20 \mathrm{kHz}$ high-speed photography was used for an improved estimate of the bubble velocity and acceleration. These images were analysed with the same method as described for the 3 $\mathrm{kHz}$ images. The results obtained from both frame rates for bubble radius, velocity and acceleration for both initial pressures are shown in Figures 5.12 and 5.13. The bubble radius is non-dimensionalised with the equivalent equilibrium bubble radius at ambient pressure. The bubble velocity is non-dimensionalised based on the Rayleigh collapse velocity:

$$
\dot{R}_{R}=\sqrt{\frac{2}{3} \frac{p_{v}-p_{\infty}}{\rho}\left[1-\left(\frac{R_{M}}{R}\right)^{3}\right]+\frac{2}{3-3 k} \frac{p_{g} R_{g}^{3 k}}{\rho}\left[\frac{1}{R^{3 k}}-\frac{R_{M}^{3-3 k}}{R^{3}}\right]}
$$


where $p_{v}$ is the vapour pressure, $p_{\infty}$ is the ambient pressure, $\rho$ is the water density, $R_{M}$ is the maximum equivalent spherical bubble radius, $R_{g}$ is the equivalent initial bubble radius (based on the firing volume), $p_{g}$ is the initial airgun pressure, and $k$ is the polytropic index, taken as the adiabatic index, 1.4. $R$ is the bubble radius, and in this case is taken as $R_{g}$.

From this, an acceleration term can be derived which is used to non-dimensionalise the bubble acceleration:

$$
\ddot{R}_{R}=\frac{1}{2 \sqrt{\dot{R}}}\left[\left[2 \frac{p_{v}-p_{\infty}}{\rho} \frac{R_{M}^{3}}{R^{4}}\right]+\frac{2}{1-k} \frac{p_{g} R_{g}^{3 k}}{\rho}\left[\frac{-k}{R^{3 k+1}}+\frac{R_{M}^{3-3 k}}{R^{4}}\right]\right]
$$

The pressure field is non-dimensionalised with the difference between initial and ambient pressure, and time is non-dimensionalised with Minneart's equation for natural bubble frequency, given by:

$$
f_{0}=\frac{1}{2 \pi r_{0}} \sqrt{\frac{3 k p_{\infty}}{\rho}}
$$

where $f_{0}$ is the natural bubble frequency and $r_{0}$ is an equivalent equilibrium bubble radius at ambient pressure.

From the Rayleigh equation, an increase in initial bubble pressure by a factor of 2 increases the maximum bubble radius by approximately 1.28. From analysis of the shadowgraph photography, the maximum bubble radius (in the $\mathrm{x}-\mathrm{y}$ plane) from 50 bar initial airgun pressure is $0.101 \mathrm{~m}$, and from 100 bar initial pressure is $0.134 \mathrm{~m}$; an increase of about 1.32. The maximum velocity achieved by both of the bubbles is approximately $20 \mathrm{~ms}^{-1}$.

Examining the average bubble radius, velocity and acceleration with respect to the field pressure response shows that the pressure pulse just after the initial shock but before the first pressure peak caused by the bubble collapse corresponds with the second velocity and acceleration peaks and the point just after the shoulder in the bubble radius. This correlation suggests that the pressure pulse is due to the secondary issuing jet penetrating the surface of the initially forming bubble as described in Section 5.3.1. No work has been found reporting this behaviour in the pressure waveforms produced by seismic airguns or explosives which may indicate that the formation of the secondary jet is attributable to the design of this specific airgun. 

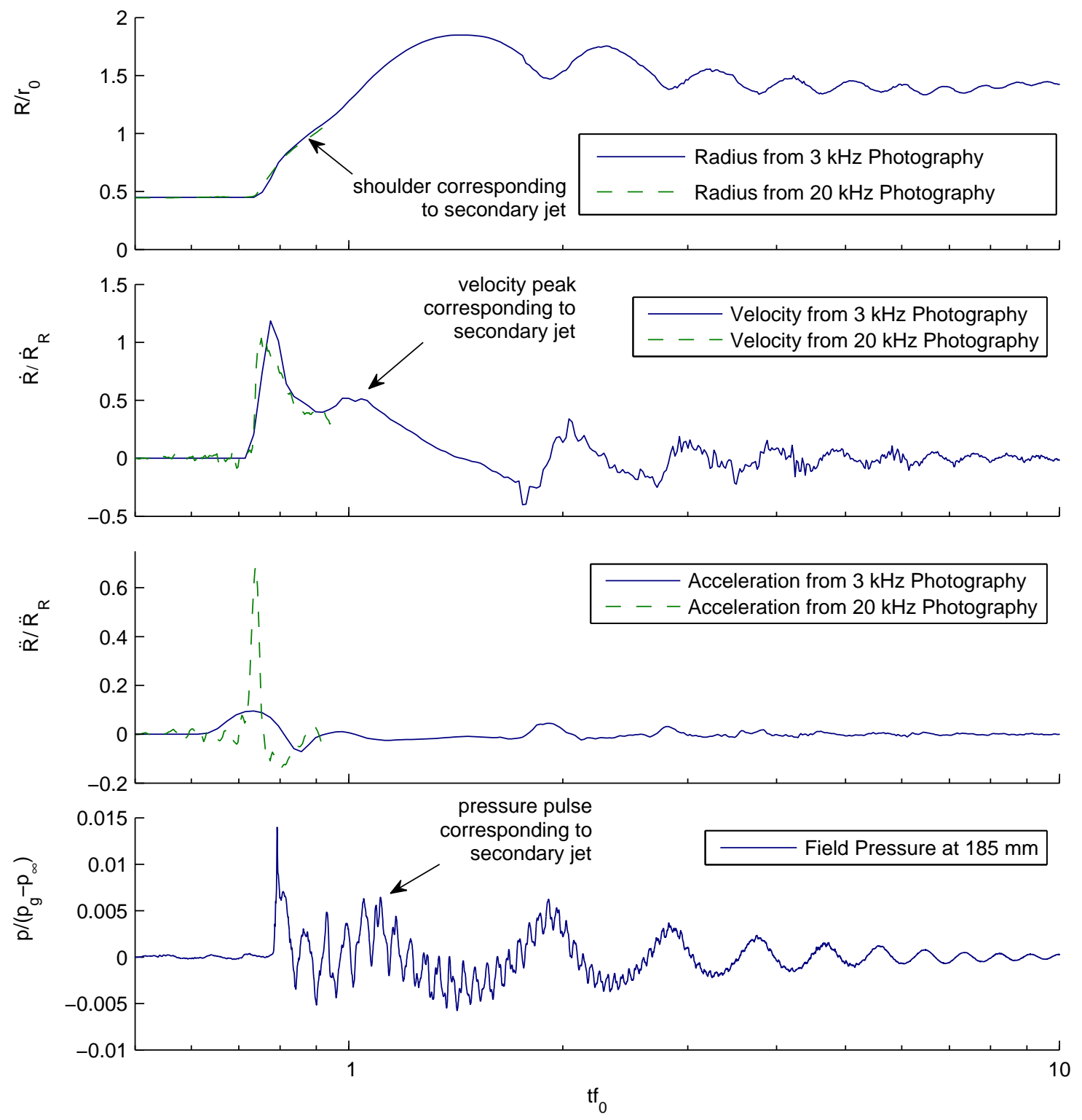

Figure 5.12: Bubble radius, velocity and acceleration from photography at frame rates of $3 \mathrm{kHz}$ and $20 \mathrm{kHz}$ for 50 bar initial airgun pressure with corresponding free-field pressure response. The bubble radius for both frame rates match well. The shoulder in the bubble radius, and second velocity and acceleration peaks are due to the secondary jet discussed in Section 5.3.1. The average radius is calculated from 10 high-speed image sequences for each frame rate and is non-dimensionalised with the equivalent equilibrium bubble radius at ambient pressure. The field pressure response is of one of these firings, aligned accordingly in time and non-dimensionalised with the difference between initial and ambient pressure. Time has been non-dimensionalised with Minneart's frequency. 

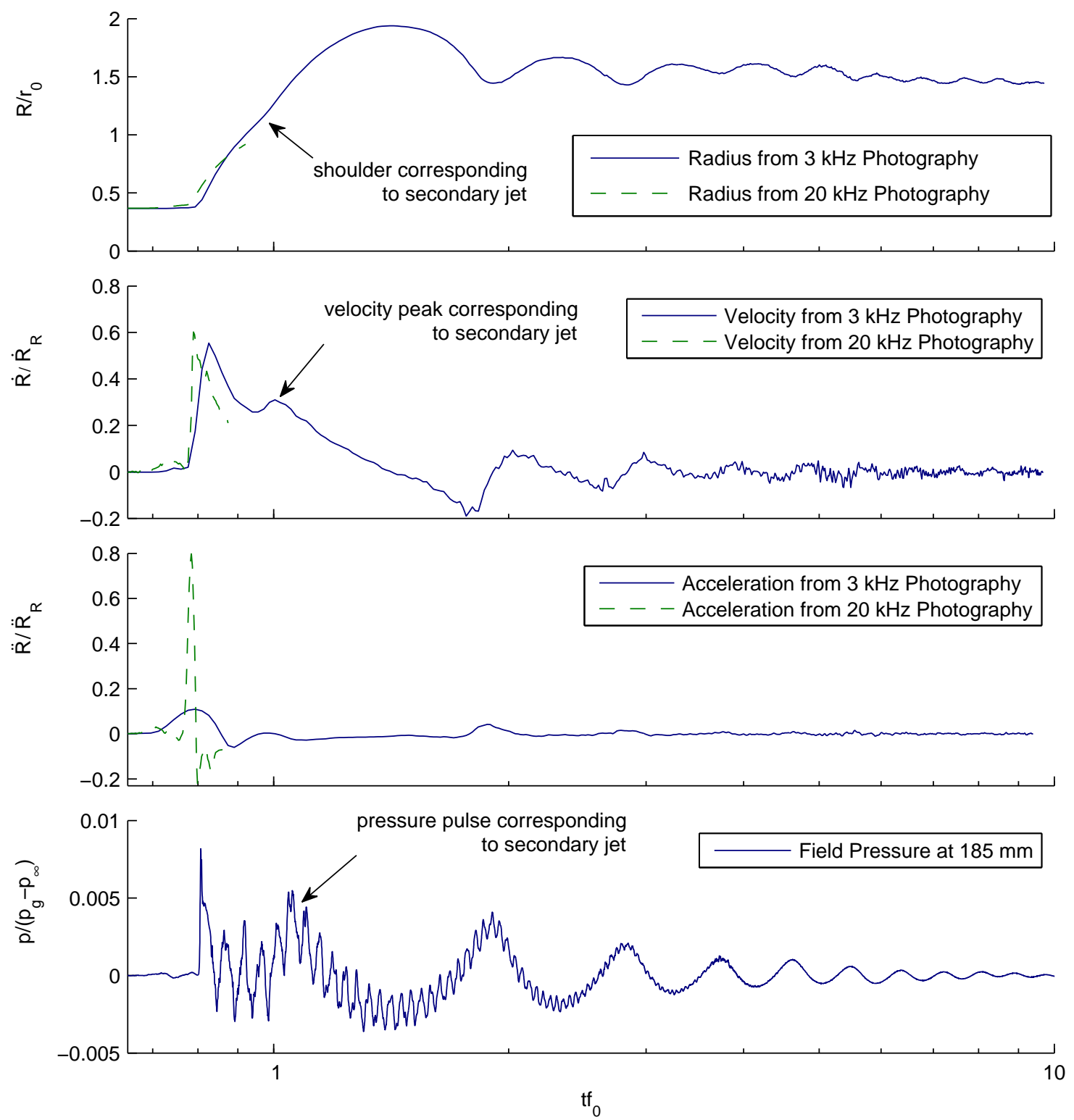

Figure 5.13: Bubble radius, velocity and acceleration from photography at frame rates of $3 \mathrm{kHz}$ and $20 \mathrm{kHz}$ for 100 bar initial airgun pressure with corresponding free-field pressure response. The bubble radius for both frame rates match reasonably. The shoulder in the bubble radius, and second velocity and acceleration peaks are due to the secondary jet discussed in Section 5.3.1. The average radius is calculated from 10 high-speed image sequences for each frame rate and is non-dimensionalised with the equivalent equilibrium bubble radius at ambient pressure. The field pressure response is of one of these firings, aligned accordingly in time and non-dimensionalised with the difference between initial and ambient pressure. Time has been non-dimensionalised with Minneart's frequency. 


\subsection{Comparison with Modified Gilmore Equation}

\subsubsection{The Modified Gilmore Equation}

Additional factors to the Gilmore equation were proposed in [19] (Chapter 3) and this model has been used to predict the radius and velocity of the model-scale airgun. The equations are solved using the classical fourth order Runge-Kutta method. The Gilmore equation (with an alternative pressure calculation definition) is given by:

$$
R \ddot{R}\left(1-\frac{\dot{R}}{c}\right)+\frac{3}{2} \dot{R}^{2}\left(1-\frac{\dot{R}}{3 c}\right)=H\left(1+\frac{\dot{R}}{c}\right)+\frac{R \dot{H}}{c}\left(1-\frac{\dot{R}}{c}\right)
$$

where

$$
\begin{gathered}
H=n\left(\frac{p_{\infty}+B}{(n-1) \rho}\right)\left[\left(\frac{p+B}{p_{\infty}+B}\right)^{\frac{n-1}{n}}-1\right] \\
c=c_{\infty}\left[\left(\frac{p+B}{p_{\infty}+B}\right)^{\frac{n-1}{2 n}}\right] \\
p=\frac{m R_{G} T}{V}+p_{v}-\frac{2 S}{R}-4 \mu \frac{\dot{R}}{R} \\
c_{\infty}=\sqrt{n\left(\frac{p_{\infty}+B}{\rho}\right)}
\end{gathered}
$$

$H$ is the enthalpy difference between the liquid at pressure $p$ (at the bubble wall) and the ambient pressure $p_{\infty}, c$ is the local speed of sound and $B$ and $n$ are constants used to calculate the speed of sound and enthalpy, and taken as $3 \times 10^{-8}$ and 7 , respectively. Over dots denote differentiation with respect to time. $m$ is the bubble mass, $R_{G}$ is the gas constant, $T$ is the bubble temperature, $V$ is the bubble volume, $S$ is the surface tension and $\mu$ is an effective viscosity which accounts for turbulent motion at the bubble wall, given by:

$$
\mu=\mu_{d y n}(1+K R e)
$$

where $\mu_{d y n}$ is the dynamic viscosity and $K$ is a constant, about 0.02 .

The bubble gas mass, volume and temperature, used for calculating the pressure, $p$, in Equation 5.8, are affected by a number of phenomena which are calculated as follows.

The bubble gas mass, $m$, is affected by the mass throttling through the ports given by:

$$
\dot{m}=A_{o} \sqrt{\frac{p_{G} m_{G}}{V_{G}} \frac{2 k}{k-1}\left[\left(\frac{p}{p_{G}}\right)^{\frac{2}{k}}-\left(\frac{p}{p_{G}}\right)^{\frac{k+1}{k}}\right]}
$$


where $p$ is limited to the sonic value, $p_{G}^{*}$ :

$$
p_{G}^{*}=p_{G}\left(\frac{2}{k+1}\right)^{\frac{k}{k-1}}
$$

$A_{o}$ is the orifice area and the subscript $G$ indicates the value inside the airgun chamber. The volume of the airgun body, $V_{A G}$, is accounted for when calculating the bubble volume, $V$, which is given by:

$$
V=\frac{4}{3} \pi R^{3}-V_{A G}
$$

The bubble temperature, $T$, is affected by the latent heat of vapour mass transfer and classical heat transfer, which are given by:

$$
\dot{Q}_{L}=\dot{m}_{v} L
$$

for the heat transfer due to latent heat, where $L$ is the latent heat of vaporization, and $\dot{m}_{v}$ is the vapour mass transfer, and

$$
\dot{Q}_{C}=\Delta T A \frac{\kappa}{d}
$$

for the classical heat transfer, where $\Delta T$ is the difference in temperature across the bubble wall, $A$ is the surface area of the bubble, $\kappa$ is the thermal conductivity of the interface and $d$ is the thickness of the thermal boundary layer.

The pressure field at a distance $r$ from the bubble is calculated according to Gilmore's model:

$$
p-p_{\infty}=\rho\left(\frac{y}{r}-\frac{u_{l}^{2}}{2}\right)+\frac{\rho}{2 c_{\infty}^{2}}\left(\frac{y}{r}-\frac{u_{l}^{2}}{2}\right)^{2}
$$

where,

$$
\begin{gathered}
u_{l}=\frac{y}{c_{\infty} r}+\frac{K_{3} y^{2}}{c_{\infty}^{3} r^{2}}\left(1-\frac{y}{c_{\infty}^{2} r}+\frac{K_{3}^{2} y^{4}}{2 c_{\infty}^{8} r^{4}}\right) \\
y=R\left(H+\frac{\dot{R}^{2}}{2}\right) \\
K_{3}=\frac{c_{\infty}^{3} R^{2} \dot{R}}{y^{2}}\left(1-\frac{\dot{R}^{2}}{2 c_{\infty}^{2}}\right)-\frac{c_{\infty}^{2} R}{y}\left(1-\frac{\dot{R}}{c_{\infty}}\right)
\end{gathered}
$$

where $p_{l}$ and $u_{l}$ are the pressure and velocity of the liquid at a distance $r$ from the bubble, and $y$ and $K_{3}$ are constants along any outgoing characteristic. 
The corresponding time co-ordinate for the pressure field is given by:

$$
t=t_{R}+\left(\frac{r-R}{c_{\infty}}\right)\left(1-\frac{\dot{R} R}{c_{\infty} r}\right)
$$

where $t_{R}$ is the time at which the characteristic started at the bubble wall $(r=R)$.

The parameters used in the model for this case are given in Table 5.1.

\begin{tabular}{lc}
\hline Firing air volume & $14.5 \mathrm{~cm}^{3}$ \\
Equivalent initial radius & $0.015 \mathrm{~m}$ \\
Airgun body volume & $65.4 \mathrm{~cm}^{3}$ \\
Throttling area & $628 \mathrm{~mm}^{3}$ \\
\hline
\end{tabular}

Table 5.1: Table of parameters used in the modified Gilmore equation to predict the bubble behaviour of the model-scale airgun.

\subsubsection{Comparison of Analytical and Experimental Results}

An equivalent spherical radius is found based on the maximum vertical and horizontal bubble radii from the 10 shadowgraph image sequences used in Section 5.3.3 for both initial airgun pressures. The bubble velocity for the spherical radius growth is found using the method previously described. The field pressure response is from one of these 10 image sequences and is aligned in time accordingly. The radius results are nondimensionalised with the equivalent equilibrium bubble radius at ambient pressure. The bubble velocity and acceleration are non-dimensionalised based on the Rayleigh collapse velocity (Equation 5.2) and derived acceleration (Equation 5.3), respectively.

Figures 5.14 and 5.15 compare the results of the modified Gilmore equation with the experimental results. The modified Gilmore equation predicts the period and first maximum bubble size well; however, the experimental results show the equilibrium bubble radius gradually becoming larger over time. This may be explained by the break up of the bubble into many small bubbles, and the larger volume that they possess in comparison with a single bubble. The initial bubble velocity is over-predicted; however, the velocities of the following bubble pulses agree well. The magnitude of the initial shock from the airgun firing for both pressures is largely over-predicted by the equation; however, again, the following pressure peaks generated by the bubble pulses are well estimated. For the experimental data, it should be noted that the magnitude of the initial shock recorded by the hydrophone may be subject to temporal and spatial resolution limitations. Considering the model results, the magnitude of the initial shock is a function of the bubble radius growth, and over-prediction in the radial growth will result 

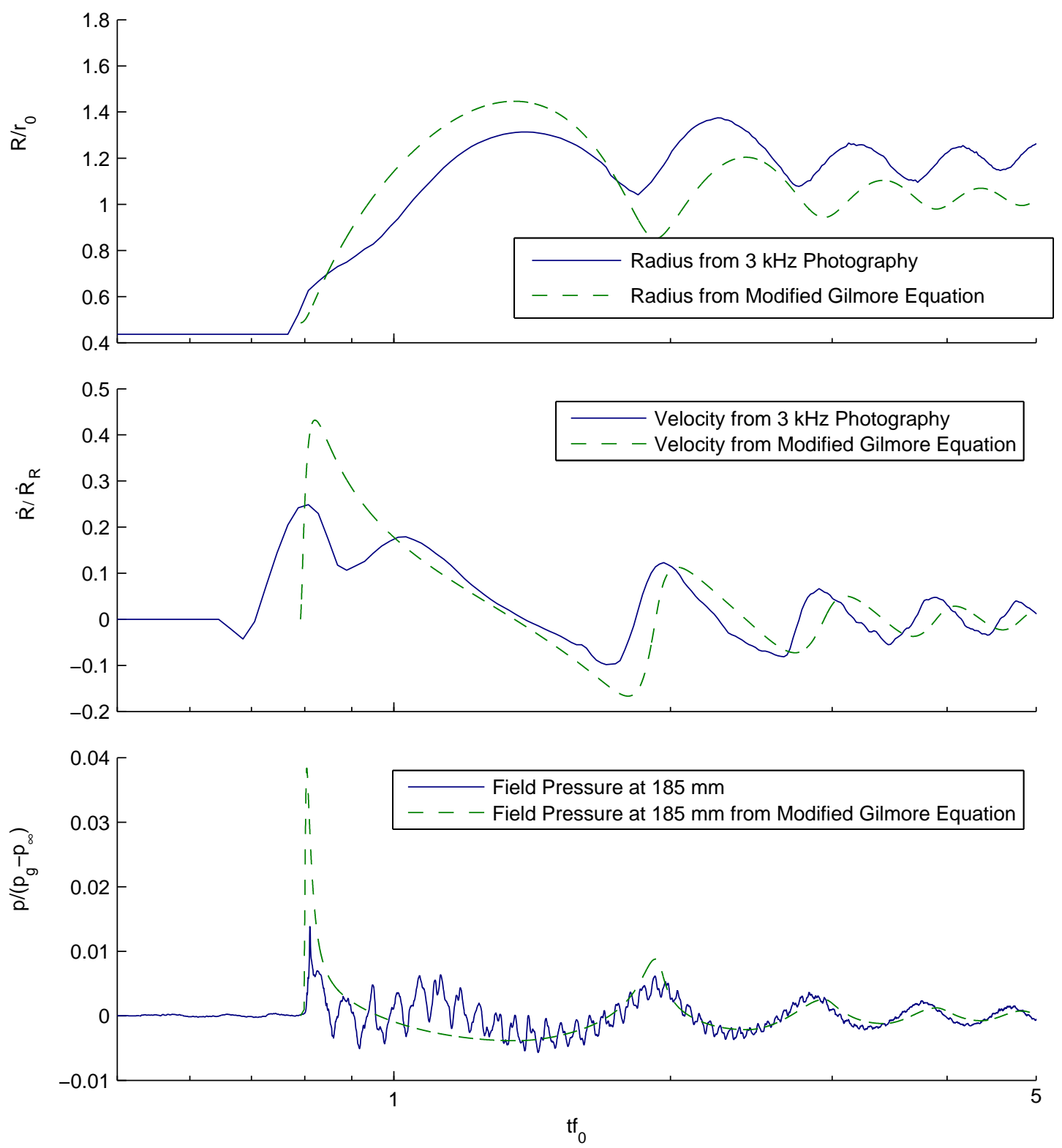

Figure 5.14: Comparison of equivalent spherical radius and velocity derived from an average of 10 high-speed shadowgraph image sequences with the results of the modified Gilmore equation for an initial airgun pressure of 50 bar. The radius is non-dimensionalised with the equivalent equilibrium bubble radius at ambient pressure. The pressure field with time at a point 185 $\mathrm{mm}$ from the airgun of one single firing, aligned accordingly in time, is also compared to that predicted by the Gilmore equation. Pressure and time are non-dimensionalised with the difference between initial and ambient pressure and Minneart's frequency, respectively 

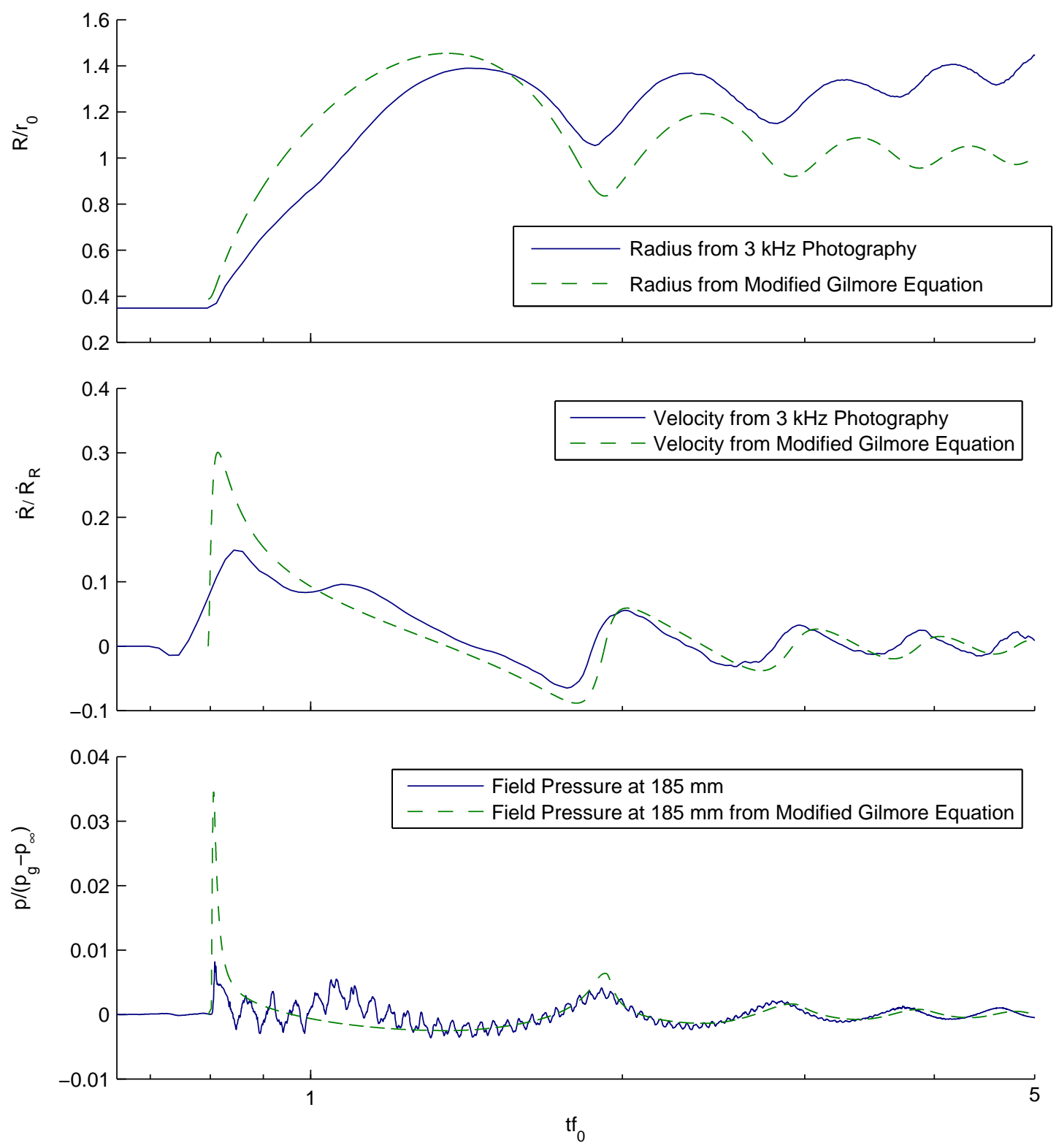

Figure 5.15: Comparison of equivalent spherical radius and velocity derived from an average of 10 high-speed shadowgraph image sequences with the results of the modified Gilmore equation for an initial airgun pressure of 100 bar. The radius is non-dimensionalised with the equivalent equilibrium bubble radius at ambient pressure. The pressure field with time at a point 185 $\mathrm{mm}$ from the airgun of one single firing, aligned accordingly in time, is also compared to that predicted by the Gilmore equation. Pressure and time are non-dimensionalised with the difference between initial and ambient pressure and Minneart's frequency, respectively 
in an over-prediction in the shock magnitude. The presence of a secondary jet in the behaviour of the airgun bubble indicates that for this airgun, not all the available energy from the release of air is radiated at the time of the airgun firing. The second increase in growth rate of the bubble is not modelled and this energy is therefore accounted for in the initial growth, causing the over-predicted values. The impulsive release of firing air is critical in the design of an effective airgun, as this produces the initial shock wave and also results in the formation of a single pulsating bubble, rather than a stream of gas that breaks up into many bubbles. This behaviour shows that the dynamics of the initial jet are a function of the shuttle dynamics and the evolving geometry of the flow passage, and an obvious area for future investigation and development.

\subsection{Conclusion}

A laboratory scale seismic airgun has been built and used to study the dynamics of the bubble produced when the airgun is fired at two different pressures. Low- and highspeed photography has been used to capture the physics of the bubble including the period of initial growth as the air is released, the point of maximum bubble radius, the first collapse and the subsequent pulses. Instabilities at the gas-water interface were noted, in particular, the presence of the Rayleigh-Taylor Instability, developed at points immediately after each bubble minimum, and also suspected immediately after firing. High resolution still images were also obtained and show the bubble surface in detail at multiple points throughout its lifetime. The interaction with nearby boundaries was investigated by firing the airgun at different standoff distances from the steel wall and the free surface. No significant interaction with the steel wall was noted for standoff distances down to 1.5 bubble radii. The presence of a free surface did affect the bubble, forming a vertical split in the bubble, similar to downward jetting, for standoff distances of 1.15 and 0.75 bubble radii. Visible changes in bubble dynamics occurred for those standoff distances for which the bubble frequency notably increased. Shadowgraph movies were used to determine the bubble radius, velocity and acceleration, which have been studied with corresponding field pressure responses. A secondary jet in the bubble growth, not previously noted in airgun pressure waveforms, is attributed to the specific design of this airgun. This indicates an inefficiency in energy release on firing the airgun and highlights the importance of airgun design in producing large magnitude initial shocks. Results from the modified Gilmore equation from [19] (Chapter 3) have been compared with the experimental data and are in fair agreement overall; however, there is a significant under-prediction of the magnitude of initial shock. This may be explained by the energy distribution between the initial shock and the observed secondary jetting. The results 
presented here provide detailed understanding of the dynamics of a bubble formed by four impulsive transient jets, as is the case for a seismic airgun firing.

\section{Acknowledgements}

This work has been funded by the Maritime Platforms Division of the Defence Science and Technology Organisation (DSTO) and the authors wish to acknowledge the support of Dr Stuart Cannon and Mr Warren Reid.

The authors also wish to acknowledge the support of Dr Bryce Pearce and invaluable technical assistance of Mr Robert Wrigley from the Australian Maritime College Cavitation Research Laboratory. 


\section{6}

\section{General Conclusion}

The work completed for this thesis was funded by DSTO and AMC in order to investigate seismic airguns and improve understanding of the unique behaviour and dynamics of the bubbles and pressure fields they produce, with a view to using airguns in shock testing of ships. The investigation is comprised of both experimental and numerical work. The development of a laboratory scale airgun and specific testing facility allowed a range of systematic experiments to be undertaken. Low- and high- speed photography was used to obtain images of spatial and temporal resolution, respectively, to study the bubble behaviour. The interfacial phenomena were captured, including the Rayleigh-Taylor Instability (RTI), as the initial jet of air was released and the bubble underwent several growth and collapse cycles. The initial shock wave and the pressure pulses generated at every bubble collapse were measured at several points in the field and on the steel wall. In addition to the wall pressure, the response of the steel wall was measured with an accelerometer. The airgun was fired at different standoff distances from the wall and the free surface to investigate the effect of nearby boundaries.

An analytical model, based on the Gilmore equation for bubble dynamics, and a numerical model, developed with the SPH method, were considered for modelling the airgun bubble. While SPH has been deemed not yet advanced enough to properly model large differences in particle densities and deformation across the gas-water interface, the Gilmore-based model was able to predict the pressure signature of a full-scale airgun (from supplied data) with good agreement. The good agreement for the initial shock was achieved through adjustment of the throttling area, as the minimum orifice size in the airgun was unknown. The predictions of the model-scale airgun bubble growth and pressure signature were also in good agreement, except for the initial shock and first bubble velocity maximum. In this case the minimum orifice size of the model-scale airgun was known, and no adjustment to this parameter was made to improve the prediction. 
In both cases, the exiting air jet through the ports may be subject to a vena contracta that results in an 'effective' orifice size.

From investigations using this model, it was determined that one of the more prevalent damping mechanisms is heat transfer across the bubble surface. It was suggested that this may be enhanced due to turbulent motion near the bubble wall, with an effective increase in surface area by a factor of 10 to be reasonably expected. As can be seen from the highspeed photography, the surface instabilities (possibly RTI) and subsequent developing turbulence of the initially emitted jet of air, and the presence of the RTI during the bubble's subsequent growth cycles confirm the large increase in surface area, enhancing any heat transfer across the bubble wall.

A second pressure pulse in the field signature of the model airgun (not normally seen in full-scale airgun signatures) was found to correspond to a second peak in the bubble velocity. This second growth period may be due to the internal flow passages in the model-scale airgun, and the double peak is likely to result in a reduced initial shock strength. As noted in the analytical model investigations, mass throttling of the air when it is released from the airgun significantly impacts the initial shock pressure and maximum bubble radius (and hence the strength of the subsequent bubble collapses). The design of air passages in the airgun must be considered for optimum efficiency, and other available commercial airguns have a sleeve type design where the air is not restricted through a number of ports.

The steel wall of the testing tank is a greatly simplified representation of a ship hull. When the airgun was fired in the centre of the tank, nominally $6-8$ bubble radii from the wall, the initial shock pressure (and reverberation reflections) were felt on the wall, but the pressure pulses from the bubble collapses were not. At the closest standoff distance, $1-3$ bubble radii from the wall, the emitted pressure pulses at each bubble collapse were still not distinct; however, some response to the bubble collapse pressure was evident. As noted in [72], the pressure felt by the steel wall, or ship, is a function of not just strength of the pressure pulse emitted at bubble collapse, but also the phasing of the wall/ship movement with the bubble frequency. The effect of stiffening the wall on the pressure signature was beyond the scope of the present study and it would certainly be of interest for future work.

In these experiments, no jetting of the bubble due to the presence of the steel wall was noted despite the airgun being fired within the interaction-limit standoff distance noted in literature. The bubble was always seen to jet onto the airgun body evidencing that the presence of the airgun body, being closer to the bubble than the external solid boundary, results in the bubble jetting towards the airgun body first. When compared with an 
underwater explosion bubble, which has no such internal object to jet onto, the bubble produced by an airgun is seemingly less likely to jet towards the nearby wall if fired at close range. The bubble was seen to interact with the free surface for small standoff distances similar to other bubbles. The effect of the orientation of the model airgun on the bubble's tendency to interact with a nearby boundary is unknown; however, when shock testing, the airgun is oriented such that the ports face the ship, and consequently jetting characteristics and tendencies would be expected to be similar to those found here. 


\section{References}

[1] P. S. Addison. The Illustrated Wavelet Transform Handbook. Taylor \& Francis Group LLC, New York, 2002.

[2] J. M. Alimi, A. Serna, C. Pastor, and G. Bernabeu. Smooth particle hydrodynamics: importance of correction terms in adaptive resolution algorithms. Journal of Computational Physics, 192:157-174, 2003.

[3] J. Barnes and P. Hut. A hierarchical $O(N \log N)$ force-calculation algorithm. Nature, 324(4):446-449, 1986.

[4] W. Benz. Smoothed particle hydrodynamics: A review. In J. Buchler, editor, The Numerical Modelling of Nonlinear Stellar Pulsations: problems and prospects, NATO ASI series: Mathematical and physical sciences, pages 269-288. Kluwer Academic Publishers, 1990.

[5] J. Best and J. R. Blake. An estimate of the Kelvin impulse of a transient cavity. Journal of Fluid Mechanics, 261:75-93, 1994.

[6] H. Bungenstock. Zur analyse und beeinflussung von siesmishen signalen, erzeugt durch pnuematische schallquellen. In Geologisches Jahrbuch, volume E. Schweizerbart Science Publishers, Stuttgart, Germany, 1973.

[7] G. Chahine, G. Frederick, C. Lambrecht, G. Harris, and H. Mair. Spark-generated bubbles as laboratory-scale models of underwater explosions and their use for validation of simulation tools. In SAVIAC Proceedings 66th Shock and Vibrations Symposium, Mississippi, U.S.A., 1995.

[8] G. L. Chahine. Experimental and asymptotic study of nonspherical bubble collapse. Applied Scientific Research, 38(1):187-197, 1982. 
[9] T. C. Chawla. The Kelvin-Helmholtz instability of the gas-liquid interface of a sonic gas jet submerged in a liquid. Journal of Fluid Mechanics, 67(3):513-537, 1975.

[10] A. Colagrossi and M. Landrini. Numerical simulation of interfacial flows by smoothed particle hydrodynamics. Journal of Computational Physics, 191(2):448475, 2003.

[11] J. A. Cook, A. M. Gleeson, R. M. Roberts, and R. L. Rogers. A spark-generated bubble model with semi-empirical mass transport. Journal of the Acoustical Society of America, 101(4):1908-1920, 1997.

[12] A. Corbishdale and W. Reid. Air gun free field pressure analysis. Technical Report DSTO-TR-2506, DSTO, 2010.

[13] F. Cox, A. Pearson, J. R. Blake, and S. R. Otto. Comparison of methods for modelling the behaviour of bubbles produced by marine seismic airguns. Geophysical Prospecting, 52(5):451-477, 2004.

[14] Z. Dai, B. Wang, L. Qi, and H. Shi. Experimental study on hydrodynamic behaviours of high-speed gas jets in still water. Acta Mechanica Sinica, 22:443448, 2006.

[15] K. de Graaf. Smoothed particle hydrodynamics of underwater explosions, honours thesis, 2007.

[16] K. L. de Graaf, I. Penesis, and P. A. Brandner. Comparison of the Rayleigh-Plesset and Gilmore equations and additional aspects for the modelling of seismic airgun bubble dynamics. In 18th Australasian Fluid Mechanics Conference, Launceston, Australia, 2012.

[17] K. L. de Graaf, P. A. Brandner, and I. Penesis. The pressure field generated by a seismic airgun. submitted to Experimental Thermal and Fluid Science, 2013.

[18] K. L. de Graaf, P. A. Brandner, and I. Penesis. Bubble dynamics of a seismic airgun. submitted to Experiments in Fluids, 2013.

[19] K. L. de Graaf, I. Penesis, and P. A. Brandner. Modelling of seismic airgun bubble dynamics and pressure field using the Gilmore equation with additional damping factors. submitted to Journal of Ocean Engineering, 2013.

[20] W. H. Dragoset. A comprehensive method for evaluating the design of airguns and airgun arrays. In Offshore Technology Conference, pages 75-84, Houston, Texas, 1984. 
[21] J. Dubinski. A parallel tree code. New Astronomy, 1(2):133-47, 1996.

[22] M. Felli, S. Grizzi, and M. Falchi. Hydroacoustic and hydrodynamic analysis of a propeller-rudder configuration by pressure signal wavelet decomposition and optical techniques. In Third International Symposium on Marine Propulsors, pages 373380, Launceston, Australia, 2013.

[23] J. P. Franc and J. M. Michel. Fundamentals of Cavitation. Kluwer Academic Publishers, Dordrecht, The Netherlands, 2004.

[24] D. L. Frost. Dynamics of explosive boiling of a droplet. Physics of Fluids, 31(9): 2554-2561, 1988.

[25] D. L. Frost and B. Sturtevant. Effects of ambient pressure on the instability of a liquid boiling explosively at the superheat limit. Journal of Heat Transfer, 108: 418-424, 1986.

[26] S. Fujikawa and T. Akamatsu. Effects of the non-equilibrium condensation of vapour on the pressure wave produced by the collapse of a bubble in a liquid. Journal of Fluid Mechanics, 97(3):481-512, 1980.

[27] F. R. Gilmore. The growth or collapse of a spherical bubble in a viscous compressible liquid. Technical Report No. 26-4, California Institute of Technology, 1952.

[28] R. A. Gingold and J. J. Monaghan. Smoothed particle hydrodynamics: theory and application to non-spherical stars. Monthly Notices of the Royal Astronomical Society, 181:375-389, 1977.

[29] K. Gong. Water entry of a wedge based on SPH model with an improved boundary treatment. Journal of Hydrodynamics, 21(6):750-757, 2009.

[30] R. J. Goozee and P. A. Jacobs. Distributed and shared memory parallelism with a smoothed particle hydrodynamics code. Australian Mathematical Society, 44:C202C228, 2003.

[31] L. Hernquist and N. Katz. TreeSPH: A unification of SPH with the hierarchical tree method. The Astrophysical Journal Supplement Series, 70:419-446, 1989.

[32] C. Herring. Theory of the pulsations of the gas bubble produced by an underwater explosion. In Underwater Explosion Research: v. 2, 1950. Officer of Naval Research, Dept of the Navy, 1949. 
[33] W. Hong, D. H. House, and J. Keyser. Adaptive particles for incompressible fluid simulation. The Visual Computer, 24(7):535-543, 2008.

[34] C.-T. Hsiao and G. L. Chahine. Air gun source model using 3DynaFS PhantomCloud. In 81st Shock and Vibration Symposium, Orlando, Florida, 2010.

[35] C. F. Hung and J. J. Hwangfu. Experimental study of the behaviour of mini-charge underwater explosion bubbles near different boundaries. Journal of Fluid Mechanics, 651:55-80, 2010.

[36] R. C. Johnston. Performance of 2000 and 6000 psi airguns: Theory and experiment. Geophysical Prospecting, 28:700-715, 1980.

[37] R. C. Johnston. Development of more efficient airgun arrays: Theory and experiment. Geophysical Prospecting, 30(6):752-773, 1982.

[38] D. D. Joseph. Cavitation and the state of stress in a flowing liquid. Journal of Fluid Mechanics, 366:367-378, 1998.

[39] M. B. Kennel. KDTREE 2: Fortran and C++ software to efficiently search for near neighbours in multi-dimensional Euclidean space. http://arXiv:physics/0408067v2 [physics.dat-an], 2004.

[40] L. E. Kinsler, A. R. Frey, A. B. Coppens, and J. V. Sanders. Fundamentals of Acoustics. John Wiley \& Sons, Inc., Canada, third edition, 1982.

[41] S. Kitsionas and A. Whitworth. Smoothed particle hydrodynamics with particle splitting, applied to self-gravitating collapse. Monthly Notices of the Royal Astronomical Society, 330(1):129-136, 2002.

[42] E. Klaseboer, K. C. Hung, C. Wang, C. W. Wang, B. C. Khoo, P. Boyce, S. Debono, and H. Charlier. Experimental and numerical investigation of the dynamics of an underwater explosion bubble near a resilient/rigid structure. Journal of Fluid Mechanics, 537:387-413, 2005.

[43] J. Langhammer and M. Landrø. Temperature effects on airgun signatures. Geophysical Prospecting, 41(6):737-750, 1993.

[44] J. Langhammer and M. Landrø. Experimental study of viscosity effects on air-gun signatures. Geophysics, 58(12):1801-1808, 1993.

[45] J. Langhammer and M. Landrø. High-speed photography of the bubble generated by an airgun. Geophysical Prospecting, 44(1):153-173, 1996. 
[46] J. Langhammer, S. Graciet, I. Vik, M. Espeland, and O. J. Lokberg. Holographic studies of the bubble generated by a seismic airgun. Journal of the Acoustical Society of America, 97(1):362-369, 1995.

[47] M. Lastiwka, N. Quinlan, and M. Basa. Adaptive particle distribution for smoothed particle hydrodynamics. International Journal for Numerical Methods in Fluids, 47: 1403-1409, 2005.

[48] M. Lastiwka, M. Basa, and N. Quinlan. Permeable and non-reflecting boundary conditions in SPH. International Journal for Numerical Methods in Fluids, 61: 709-724, 2009.

[49] W. Lauterborn and H. Bolle. Experimental investigations of cavitation-bubble collapse in the neighbourhood of a solid boundary. Journal of Fluid Mechanics, 72(2):391-401, 1975.

[50] R. Laws, M. Landrø, and L. Amundsen. An experimental comparison of three direct methods of marine source signature estimation. Geophysical Prospecting, 46 (4):353-389, 1998.

[51] R. M. Laws, L. Hatton, and M. Haartsen. Computer modelling of clustered airguns. First Break, 8(9):331-338, 1990.

[52] T. G. Leighton. The Acoustic Bubble. Academic Press, California, 1994.

[53] G. F. Li, M. Q. Cao, H. L. Chen, and C. Z. Ni. Modeling air gun signatures in marine seismic exploration considering multiple physical factors. Applied Geophysics, 7(2): 158-165, 2010.

[54] O. Lindau and W. Lauterborn. Cinematographic observation of the collapse and rebound of a laser-produced cavitation bubble near a wall. Journal of Fluid Mechanics, 479:327-348, 2003.

[55] G. R. Liu and M. B. Liu. Smoothed Particle Hydrodynamics: A Meshfree Particle Method. World Scientific Publishing Co. Pte. Ltd, Singapore, 2003.

[56] G. R. Liu, M. B. Liu, K. Y. Lam, and Z. Zong. Simulation of the explosive detonation process using SPH methodology. In K. J. Bathe, editor, Computational Fluid and Solid Mechanics, pages 323-326. Elsevier, 2001.

[57] M. B. Liu, G. R. Liu, and K. Y. Lam. Investigations into water mitigation using a meshless particle method. Shock Waves, 12(3):181-195, 2002. 
[58] M. B. Liu, G. R. Liu, Z. Zong, and K. Y. Lam. Computer simulation of high explosive explosion using smoothed particle hydrodynamics methodology. Computers and Fluids, 32(3):305-322, 2002.

[59] M. B. Liu, G. R. Liu, K. Y. Lam, and Z. Zong. Smoothed particle hydrodynamics for numerical simulation of underwater explosion. Computational Mechanics, 30: 106-118, 2003.

[60] O. M. F. R. S. Lord Rayleigh. Viii. on the pressure developed in a liquid during collapse of a spherical cavity. Philisophical Magazine Series 6, 34(200):94-98, 1917.

[61] S. Menon and M. Lal. On the dynamics and instability of bubbles formed during underwater explosions. Experimental Thermal and Fluid Science, 16(4):305-321, 1998.

[62] J. J. Monaghan. On the problem of penetration in particle methods. Journal of Computational Physics, 82(1):1-15, 1989.

[63] J. J. Monaghan. Smoothed particle hydrodynamics. Annual Review of Astronomy and Astrophysics, 30:543-574, 1992.

[64] J. J. Monaghan. Smoothed particle hydrodynamics. Reports on Progress in Physics, 68:1703-1759, 2005.

[65] J. P. Morris. A study of the stability properties of smooth particle hydrodynamics. Publications of the Astronomical Society of Australia, 13:97-102, 1996.

[66] R. P. Nelson and C. B. Papaloizou. Three-dimensional hydrodynamic simulations of collapsing prolate clouds. Monthly Notices of the Royal Astronomical Society, 265: 905-920, 1993.

[67] R. P. Nelson and C. B. Papaloizou. Variable smoothing lengths and energy conservation in smoothed particle hydrodynamics. Monthly Notices of the Royal Astronomical Society, 270:1-20, 1994.

[68] C. Z. Ni, H. L. Chen, J. Liu, Y. Q. Ye, H. X. Niu, M. Q. Cao, and Y. Liu. Parameter optimization in air-gun type-dependent signature modelling. In 73rd EAGE Conference \& Exhibition incorporating SPE EUROPEC, Vienna, Austria, 2011.

[69] H. Oke. Conduct and logistics of an airgun trial of a decommissioned type 42 destroyer. In 79th Shock and Vibration Symposium. SAVIAC, 2008. 
[70] J. W. Pritchett. An evaluation of various theoretical models for underwater explosion bubble pulsation. Technical report, Information Research Associates, 1971.

[71] W. Reid, L. de Yong, P. Elischer, and M. Skeen. Underwater shock testing with seismic air guns. In 8th International Conference on Shock 85 Impact Loads on Structures, Adelaide, Australia, 2009. Defence Science and Technology Organisation.

[72] W. D. Reid. Response of surface ships to underwater explosions. Technical Report DSTO-GD-0109, Defence Science and Technology Organisation, 1996.

[73] J. D. Romeel Dave and L. Hernquist. Parallel treeSPH. New Astronomy, 2:277-297, 1997.

[74] M. H. Safar. Efficient design of airgun arrays. Geophysical Prospecting, 24(4): 773-787, 1976.

[75] R. Schulze-Gattermann. Physical aspects of the airpulser as a seismic energy source. Geophysical Prospecting, 20(1):155-192, 1972.

[76] J. E. Shepherd. Interface effects in underwater explosions, conventional weapons underwater explosions, Office of Naval Research. Technical report, Georgia Institute of Technology, 1988.

[77] J. E. Shepherd and B. Sturtevant. Rapid evaporation at the superheat limit. Journal of Fluid Mechanics, 121:379-402, 1982.

[78] A. Shima, K. Takayama, and Y. Tomita. Mechanisms of the bubble collapse near a solid wall and the induced impact pressure generation. Reports of the Institute High Speed Mechanics, Tohuku University, 48:77-97, 1984.

[79] J. W. Swegle and S. W. Attaway. On the feasibility of using smoothed particle hydrodynamics for underwater explosion calculations. Computational Mechanics, 17:151-168, 1995.

[80] P. Thompson, G. Colliar, D. Manley, and H. Peterson. The use of seismic airguns for affordable whole ship shock testing. In 10th International Naval Engineering Conference and Exhibition. Imarest, 2010.

[81] Y. Tomita and T. Kodama. Interaction of laser-induced cavitation bubbles with composite surfaces. Journal of Applied Physics, 94(5):2809-2816, 2003. 
[82] C. Torrence and G. P. Compo. A practical guide to wavelet analysis. Bulletin of the American Meteorological Society, 79(1):61-78, 1998.

[83] S. Vaage and B. Ursin. Computation of signatures of linear airgun arrays. Geophysical Prospecting, 35(3):281-287, 1987.

[84] S. Vaage, K. Haugland, and T. Utheim. Signatures from single airguns. Geophysical Prospecting, 31(1):87-97, 1983.

[85] K. Vokurka. Comparison of rayleigh's, herring's and gilmore's models of gas bubbles. Acustica, 59(3):214-219, 1986.

[86] Weidlinger

Associates

Ltd and P. R. Thompson. Shock testing of naval vessels using seismic airgun arrays US 6662624 B1. http://www.google.com.au/patents/US6662624, 2003.

[87] C. Weiland and P. P. Vlachos. Round gas jets submerged in water. International Journal of Multiphase Flow, 48:46-57, 2013.

[88] F. M. White. Fluid Mechanics, 5th Edition. McGraw Hill, New York, NY, 2003.

[89] A. Ziolkowski. A method for calculating the output pressure waveform from an airgun. Geophysical Journal of the Royal Astronomical Society, 21:137-161, 1970.

[90] A. Ziolkowski. An airgun model which includes heat-transfer and bubble interactions. In SEG Annual Meeting, pages 187-189, Dallas, Texas, 1982. 


\section{Appendix A}

\section{Australasian Fluid Mechanics Conference Paper: \\ Comparison of the Rayleigh-Plesset and Gilmore Equations and Additional Aspects for the Modelling of Seismic Airgun Bubble Dynamics}

This paper was presented at the (peer-reviewed) $18^{\text {th }}$ Australasian Fluid Mechanics Conference, 3-7 December 2012.

The citation for the conference paper is:

de Graaf, K.L., Penesis, I., Brandner, P.A., Comparison of the Rayleigh-Plesset and Gilmore Equations and Additional Aspects for the Modelling of Seismic Airgun Bubble Dynamics, Proceedings of the $18^{\text {th }}$ Australasian Fluid Mechanics Conference, 3-7 December, Launceston Tasmania, Australia 


\title{
Comparison of the Rayleigh-Plesset and Gilmore Equations and Additional Aspects for the Modelling of Seismic Airgun Bubble Dynamics
}

\author{
K. L. de Graaf, I. Penesis and P. A. Brandner \\ Australian Maritime College \\ University of Tasmania, Tasmania, 7250, Australia
}

\begin{abstract}
Seismic airguns are commonly used in geophysical exploration. More recently, they are also being used as an alternative to underwater explosions for the shock testing of defence vessels. The study of the dynamics of the bubble produced by a seismic airgun is beneficial in understanding the resultant pressure field and shockwave.

The Rayleigh-Plesset and Gilmore equations for modelling spherical bubble dynamics are compared for the expansion of an initially highly pressurised gas bubble. The relationship between initial gas pressure and both the first maximum bubble radius and the first period of oscillation are presented. The initial gas pressure is non-dimensionalised against hydrostatic pressure and studied over a range of $1-50$. The separate contributions of presence of the airgun body, mass throttling, effective viscosity and heat diffusion to the first maximum radius and period are modelled and discussed. The effects of evaporation and condensation at the bubble wall are also considered.
\end{abstract}

\section{Introduction}

The Royal Australian Navy is currently investigating the feasibility and advantages of employing seismic airguns for shock testing naval craft. Shock testing with seismic airguns, rather than high explosives, is less expensive, safer, and more environmentally friendly. To perform shock testing effectively, an array of airguns must be used and the interactions between the bubbles can alter the pressure fields produced. Several methods exist for calculating the interactions between bubbles in an array $[9,14]$, but all rely on a basic understanding of the parameters affecting a single airgun bubble and the pressure field and shockwave produced.

The Gilmore equation for bubble dynamics is commonly used as the underlying basis for seismic airgun bubbles and underwater explosions. Comparisons exist of this equation with other bubble models, including the well known Rayleigh-Plesset equation; however, they consider a bubble's collapse from its maximum radius rather than expansion from its minimum radius. In modelling seismic airgun bubbles it is more practical to consider the initial bubble pressure and radius, rather than the conditions at the first maximum. The present work compares the Gilmore equation to the Rayleigh-Plesset equation to confirm the use of the Gilmore equation as the basic bubble model.

Several contributions have been made to improve the numerical modelling of individual seismic airgun bubbles by considering additional factors to the basic bubble dynamics. Ziolkowski [17] used Gilmore's equation and found a polytropic index of 1.13 gave good results for the first period of oscillation; this value was also obtained by Dragoset [2] for a range of gun sizes. Shulze-Gatterman [13] emphasised the effect of the actual airgun body on the period of oscillation. Safar [12] compared the equation of a bubble to an electrical circuit and developed a model for the rise time, amplitude of the initial pulse, and period of the airgun. Johnston [7] and Dragoset [2] considered the effect of the shuttle motion and choked flow rate on the chamber pressure, with Dragoset allowing for the actual port size. Ziolkowski [18] proposed that heat transfer occurs through the latent heat released by evaporation and condensation at the bubble wall. This concept is repeated by Langhammer and Landro [8]. Laws et al. [9] consider mass transfer due to evaporation and condensation, classical heat diffusion, flow throttling and an 'effective viscosity' induced by the turbulent nature of the bubble. It is claimed that this turbulent nature also has an amplifying effect on the heat transfer across the bubble wall. Li et al [10] includes the effect of mass throttling (but not choked flow) through ports, the airgun body, heat transfer and hydrostatic pressure changes as the bubble rises through the water.

There appears to be no work that considers all of these parameters together and provides values for coefficients with a summary of the impact of each parameter on the bubble behaviour. The present work uses the Gilmore equation as the basic bubble model and considers the individual effects of the presence of the airgun body, mass throttling, effective viscosity, heat diffusion and condensation and evaporation, providing a summary of each contribution.

\section{Comparison of Rayleigh-Plesset and Gilmore Equations}

The Rayleigh-Plesset equation describes the motion of a spherical bubble in an incompressible liquid [3]. When considering bubble velocities of an appreciable order of magnitude compared with the speed of sound in water, compressibility of the liquid cannot be ignored. The Gilmore equation includes second-order compressibility terms, accounting for the loss of bubble energy due to the radiated pressure waves [5]. Both equations are commonly used to model bubble dynamics, with the Gilmore equation often used in underwater explosion applications. The Rayleigh-Plesset equation is given by:

$$
\rho\left[R \ddot{R}+\frac{3}{2} \dot{R}^{2}\right]=p_{0}\left(\frac{R_{0}}{R}\right)^{3 k}+p_{v}-\frac{2 S}{R}-4 \mu \frac{\dot{R}}{R}-p_{\infty}(t)
$$

The Gilmore equation is given by:

$$
R \ddot{R}\left(1+\frac{\dot{R}}{c}\right)+\frac{3}{2} \dot{R}^{2}\left(1-\frac{\dot{R}}{3 c}\right)=H\left(1+\frac{\dot{R}}{c}\right) \dot{H}
$$

where

$$
\begin{gathered}
H=n\left(\frac{p_{\infty}+B}{(n-1) \rho}\right)\left[\left(\frac{p+B}{p_{\infty}+B}\right)^{\frac{n-1}{n}}-1\right] \\
c=c_{\infty}\left[\left(\frac{p+B}{p_{\infty}+B}\right)^{\frac{n-1}{2 n}}\right] \\
p=p_{0}\left(\frac{R_{0}}{R}\right)^{3 k}+p_{v}-\frac{2 S}{R}-4 \mu \frac{\dot{R}}{R} \\
c_{\infty}=\sqrt{n\left(\frac{p_{\infty}+B}{\rho}\right)}
\end{gathered}
$$


$R$ is the bubble radius, $\rho$ is the water density, $p_{v}$ is the vapour pressure, $p_{\infty}$ is the hydrostatic pressure, $p_{0}$ is the initial noncondensable gas pressure, $R_{0}$ is the initial bubble radius, $k$ is the polytropic index, which varies between 1 (isothermal) and 1.4 (adiabatic) - taken as 1.4 in this work where required, $S$ is the surface tension, $\mu$ is the dynamic viscosity, $H$ is the enthalpy difference between the liquid at pressure $p$ and $p_{\infty}$, and $c_{\infty}$ is the speed of sound in water at an infinite distance from the bubble. $B$ and $n$ are constants used to calculate the local speed of sound, $c$, and enthalpy. Gilmore [5] gives $B=3000 \mathrm{~atm}$ and $n=7$. Overdots represent differentiation with respect to time.

Vokurka [15] compared these two equations for bubble collapse and determined that for amplitudes $\left(R_{\max } / R_{\text {equilibrium }}\right)$ greater than 2 , the Gilmore equation produces superior results. The first maximum radius as a function of the initial pressure for expanding bubbles is shown in figure 1 . The maximum radius is non-dimensionalised with respect to the initial radius and the initial pressure with respect to the hydrostatic water pressure. The results start to diverge for pressure ratios greater than about 3. The Gilmore equation is considered to produce the more accurate results due to the presence of the higher order terms. In

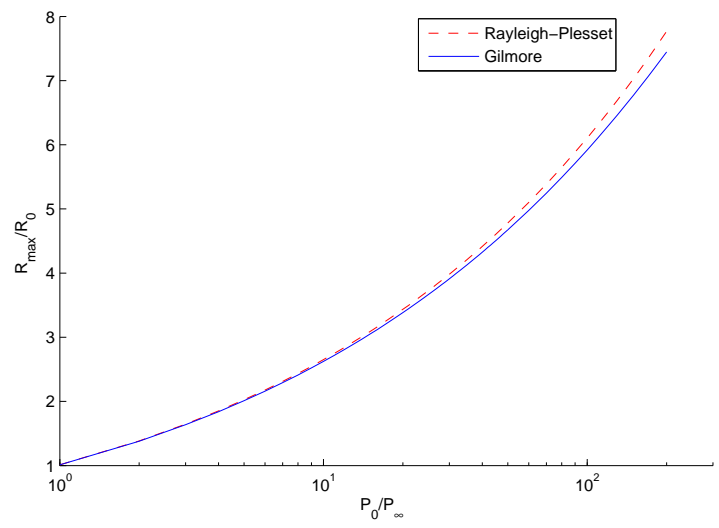

Figure 1: First maximum radius as a function of initial pressure predicted by Rayleigh-Plesset and Gilmore equations.

the study of bubble collapses, the collapse of a Gilmore bubble is fractionally (1.005) [3] longer than the collapse of a Rayleigh bubble. When modelling the bubble expansion and collapse, it is found that a Gilmore bubble has a shorter period than the Rayleigh bubble because a smaller maximum amplitude is predicted (figure 2). Due to the size of the airgun bubbles modelled, it is also found that the surface tension and dynamic viscosity terms are negligible in both solutions. For seismic airguns, the initial pressure is generally at least an order of magnitude greater than the hydrostatic pressure; therefore, while this study is interesting, the overlap between the Rayleigh-Plesset and Gilmore equations is insignificant in the study of airguns. As the Gilmore equation is considered more accurate, all further predictions will use this as the base model. The surface tension term will be ignored. Dynamic viscosity is taken as $1 \times 10^{-3}$ $\mathrm{kg} / \mathrm{ms}$ as it is required when calculating the effective viscosity and the thermal boundary layer.

\section{Additional Aspects to the Gilmore Equation}

It is well noted $[9,13,18,10]$ that while the Gilmore equation models compressibility which leads to acoustic damping, additional damping parameters must also be present in the dynamics of an airgun bubble. Typically, a real airgun bubble will have lost its energy after only a few growth/collapse cycles. Predictions with the Gilmore equation result in oscillations that persist

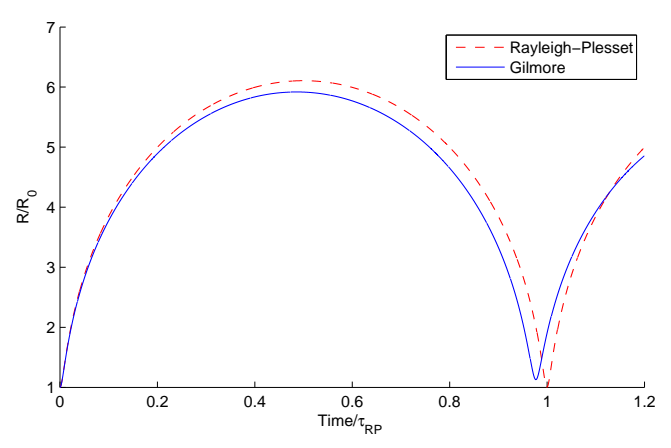

Figure 2: Comparison of predicted temporal variation of bubble radius from Rayleigh-Plesset and Gilmore equations for an initial pressure and radius of 100 bar and $0.01 \mathrm{~m}$ respectively. $\tau_{R P}$ is the collapse time for the Rayleigh-Plesset bubble.

much longer. The additional factors identified here have been modelled using the parameters of a scale model airgun; the initial bubble radius is taken as $0.014 \mathrm{~m}$, the initial pressure is 100 bar and hydrostatic pressure is 1 bar. The numerical integration is performed using a fourth order Runge-Kutta method. To incorporate mass throttling and temperature effects, the ideal gas law pressure equation (5) is replaced by:

$$
p=\frac{m R_{G} T}{V}+p_{v}
$$

where $m$ is the bubble gas mass, $R_{G}$ is the gas constant, $T$ is the bubble temperature and $V$ is the bubble volume. When the gun is fired, the air expands through four 'ports' into the surrounding water. Depending on the gun design, the air may also pass through other internal constrictions before release. These orifices throttle the flow rate and considering the pressure differences involved, choked flow conditions can be assumed. The mass flow function is [16]:

$$
\dot{m}=A \sqrt{\frac{p_{G} m_{G}}{V_{G}} \frac{2 k}{k-1}\left[\left(\frac{p}{p_{G}}\right)^{\frac{2}{k}}-\left(\frac{p}{p_{G}}\right)^{\frac{k+1}{k}}\right]}
$$

where $p$ is limited to the sonic value, $p_{G}^{*}$ :

$$
p_{G}^{*}=p_{G}\left(\frac{2}{k+1}\right)^{\frac{k}{k-1}}
$$

$A$ is the orifice area, in this model taken as $336.9 \mathrm{~cm}^{2}$, and the subscript $G$ indicates the value inside the airgun chamber. The mass throttling limits the initial maximum radius, subsequently reducing the first period and the maximum bubble pressure achieved after the first collapse (figure 3 ).

The presence of the airgun body at the bubble centre has been identified as a contributing factor to the bubble dynamics [13]. The volume of air in the bubble is:

$$
V=\frac{4}{3} \pi R^{3}-V_{A G}
$$

where $V_{A G}$ is the volume of the airgun body, in this model taken as $6.54 \times 10^{-5} \mathrm{~m}^{3}$. This volume is then used to calculate the bubble pressure, which in effect is increased at the maximum bubble radius as compared with the bubble pressure where no body is present. The reduced pressure difference between the bubble and the water reduces the intensity and velocities of the collapse; therefore, the first bubble minimum is larger, and the following oscillations less intense (figure 3). 


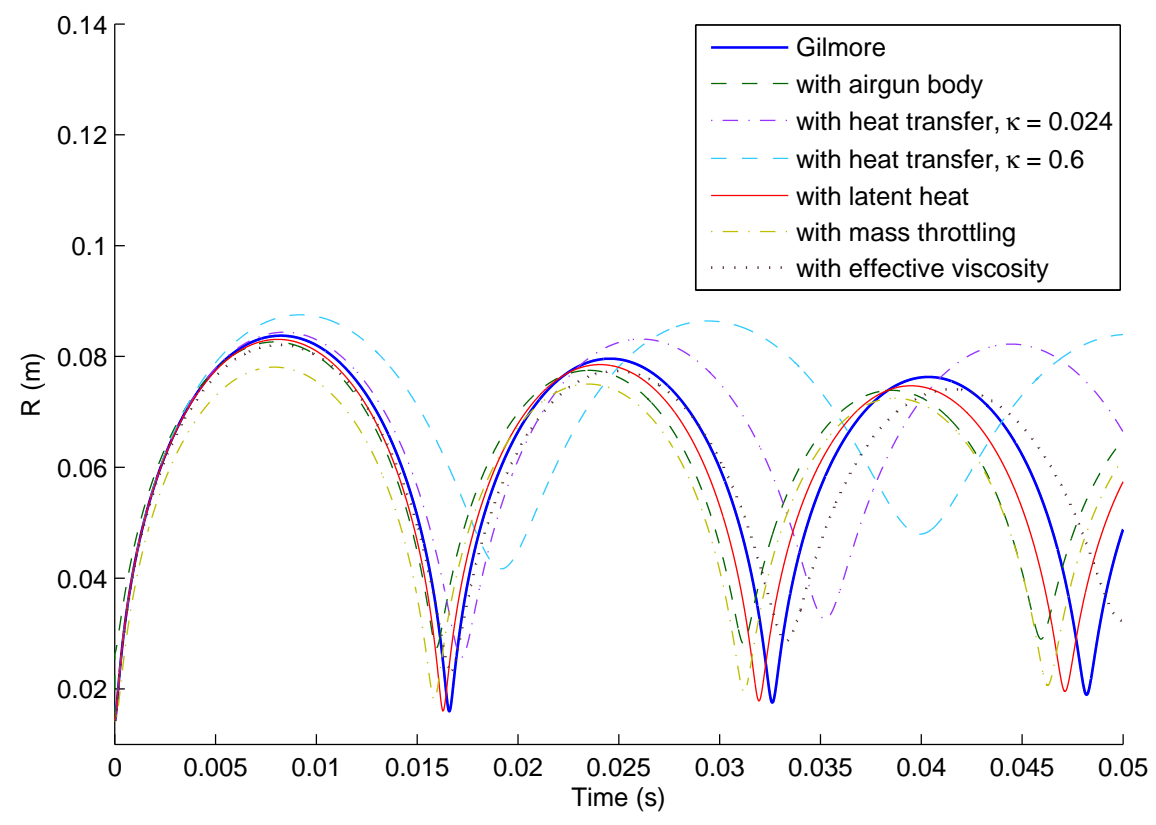

Figure 3: Effect on bubble radius of including different aspects to the Gilmore equation.

Laws et al. [9] include an effective viscosity, $\mu_{e f f}$, to account for turbulent motion at the bubble wall which occurs at large Reynolds numbers. This increases the heat transfer across the bubble wall and the viscous damping of the bubble oscillation.

$$
\mu_{e f f}=\mu(1+K R e)
$$

$K$ is a constant, about 0.02 . The Reynolds number, $R e$, is calculated based on the instantaneous bubble radius and velocity. The effect of increasing the viscosity has a small impact on the damping of the bubble (figure 3); note, when considered independently there is no heat transfer in the bubble, so this implementation only models the increased viscous damping.

Li et al [10] identified that Ziolkowski's [17] model (essentially Gilmore's model with $k$ equal to 1.13 ) results in an equilibrium temperature well below that of the surrounding water. The inclusion of heat transfer in the model results in more realistic predictions of equilibrium temperatures. Laws et al. [9] give the thickness of the thermal boundary layer as:

$$
d=4 D \operatorname{Re}^{-\frac{3}{4}} \operatorname{Pr}^{-\frac{1}{2}}
$$

where $D$ is the bubble diameter and $P r$ is the Prandtl number. The rate of heat conduction across this layer is:

$$
\dot{Q}=\Delta T A \frac{\kappa}{d}
$$

where $\Delta T$ is the difference in temperature across the bubble wall, $A$ is the surface area of the bubble and $\kappa$ is the thermal conductivity of the interface. Ni et al [11] studied a range of $\kappa / d$ values from $2000-8000 \mathrm{~W} / \mathrm{m}^{2} \mathrm{~K}$ and found correspondance with specific experimental data for a value of $4000 \mathrm{~W} / \mathrm{m}^{2} \mathrm{~K}$. Herring [6] states that the flow of heat outward is a function of the thermal conductivity of the gas. The thermal boundary layer will extend across the bubble interface, complicating the value of the thermal conductivity. Here, values for $\kappa$ of $0.6 \mathrm{~W} / \mathrm{mK}$ for water and $0.024 \mathrm{~W} / \mathrm{mK}$ for air have been considered; $d$ is updated through the calculations but is in the order of $100 \mu \mathrm{m}$. Due to turbulence, the area across which heat is conducted may be magnified - Laws et al. [9] used a factor of 10; however, in this example, the actual surface area is used. Modelling heat transfer lessens the drop in temperature as the bubble expands, allowing the bubble to reach a greater maximum radius. The temperature is also increased during collpase, which increases the pressure, arresting the collapse at a larger minimum radius. The magnitude of the following oscillations is likewise diminished (figure 3). The bubble temperature is shown in figure 4 .

In place of classical heat transfer, Ziolkowski [18] proposed that the heat transfer takes place through latent heat released by evaporation and condensation at the bubble wall. Fujikawa and Akamatsu [4] produced a detailed mathematical formulation to consider these effects. Simpler models have been used by Laws et al. [9] and Cook et al. [1]. The vapour mass transfer is:

$$
\dot{m}_{v}=\frac{\alpha_{M} A}{\sqrt{2 \pi R_{G}}}\left[\frac{p_{v}^{*}}{\sqrt{T_{w}}}-\Gamma \frac{p_{v}}{\sqrt{T}}\right]
$$

where $\alpha_{M}$ is the ratio of vapour molecules sticking to the phase interface and those impinging on it, about 0.04 [4], $A$ is the surface area of the bubble, $p_{v}^{*}$ is the equilibrium vapour pressure [4] and $T_{w}$ is the temperature of the water. $\Gamma$ is a correction factor, assumed to be 1 for these calculations. The heat flow due to the vapour mass transfer is given by:

$$
\dot{Q}=\dot{m}_{v} L
$$

where $L$ is the latent heat of vaporization, taken as $2.45 \mathrm{~J} / \mathrm{kg}$. The impact on the bubble wall dynamics due to movement of the bubble wall as a result of mass flow has been ignored, as the additional terms are assumed negligible [1]. Only the effect on bubble temperature and pressure has been considered. Modelling the mass transfer due to evaporation and condensation has a small impact on the maximum radius and period of the bubble pulses (figure 3 ). The effect on the bubble temperature is also small (figure 4) and there is no appreciable difference to the final bubble temperature. Figure 5 presents the results of including all additional factors discussed here. Solutions have been calculated for both proposed limits of $\kappa$. Increasing the surface area in equation (13) would further increase the bubble period and damping. 


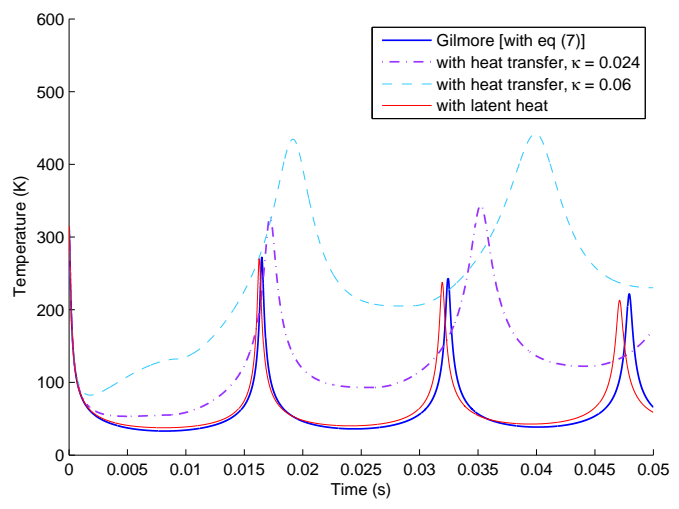

Figure 4: Effect on bubble temperature of including conductive heat transfer and heat transfer through latent heat.

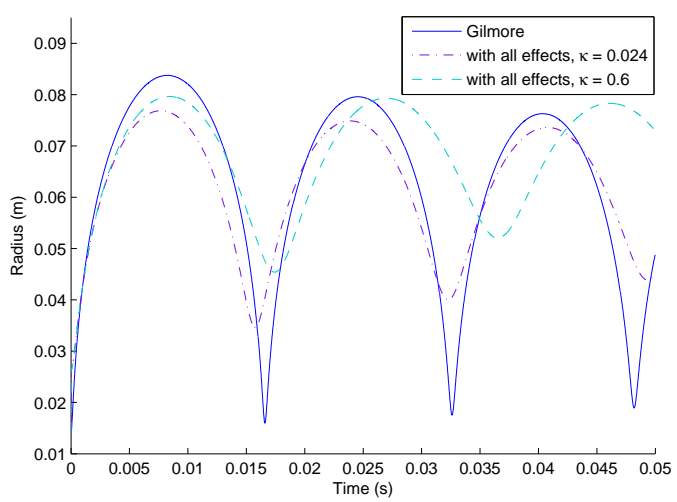

Figure 5: Combined effect on bubble radius of including all additional factors.

\section{Conclusion}

Various models have been studied that could account for differences in airgun bubble dynamics between theoretical predictions and experimental observations. From the above study, the most likely primary cause of additional damping to the seismic airgun bubble is heat transfer between the water and the air. This is potentially enhanced by an increase in bubble surface area due to turbulent motion near the bubble wall. Initial mass throttling as the air is released impacts the first maximum radius of the bubble significantly. The scale model parameters used in these numerical predictions may result in different emphasis on some factors when compared to predictions for a full scale airgun; these parameters have been chosen for comparison with experimental results. Further study will determine how the additional damping factors change with dimensional and pressure scaling.

\section{Acknowledgements}

This work has been funded by the Maritime Platforms Division of the Defence Science and Technology Organisation (DSTO) and the authors wish to acknowledge the support of Dr Stuart Cannon and Mr Warren Reid.

\section{References}

[1] Cook, J. A., Gleeson, A. M., Roberts, R. M. and Rogers, R. L., A spark-generated bubble model with semi- empirical mass transport, Journal of the Acoustical Society of America, 101, 1997, 1908-1920.

[2] Dragoset, W., A comprehensive method for evaluating the design of airguns and airgun arrays, in Offshore Technology Conference, Houston, Texas, 1984, 75-84.

[3] Franc, J. and Michel, J., Fundamentals of Cavitation, Kluwer Academic Publishers, Dordrecht, The Netherlands, 2004.

[4] Fujikawa, S. and Akamatsu, T., Effects of the nonequilibrium condensation of vapour on the pressure wave produced by the collapse of a bubble in a liquid, Journal of Fluid Mechanics, 97, 1980, 481-512.

[5] Gilmore, F., The growth or collapse of a spherical bubble in a viscous compressible liquid, Technical Report Report No. 26-4, California Institute of Technology, 1952.

[6] Herring, C., Theory of the pulsations of the gas bubble produced by an underwater explosion, in Underwater Explosion Research: v. 2, 1950, Officer of Naval Research, Dept of the Navy, 1949.

[7] Johnston, R. C., Development of more efficient airgun arrays: Theory and experiment, Geophysical Prospecting, 30, 1982, 752-773.

[8] Langhammer, J. and Landro, M., Temperature effects on airgun signatures, Geophysical Prospecting, 41, 1993, $737-750$.

[9] Laws, R. M., Hatton, L. and Haartsen, M., Computer modelling of clustered airguns, First Break, 8, 1990, 331-338.

[10] Li, G. F., Cao, M. Q., Chen, H. L. and Ni, C. Z., Modeling air gun signatures in marine seismic exploration considering multiple physical factors, Applied Geophysics, 7, 2010, 158-165.

[11] Ni, C. Z., Chen, H. L., Liu, J., Ye, Y., Niu, H., Cao, M. Q. and Liu, Y., Parameter optimization in air-gun typedependent signature modelling, in 73rd EAGE Conference \& Exhibition incorporating SPE EUROPEC, Vienna, Austria, 2011.

[12] Safar, M. H., Efficient design of airgun arrays, Geophysical Prospecting, 24, 1976, 773-787.

[13] Schulze-Gattermann, R., Physical aspects of the airpulser as a seismic energy source, Geophysical Prospecting, 20, 1972, 155-192.

[14] Vaage, S. and Ursin, B., Computation of signatures of linear airgun arrays, Geophysical Prospecting, 35, 1987, 281-287.

[15] Vokurka, K., Comparison of rayleigh's, herring's and gilmore's models of gas bubbles, Acustica, 59, 1986, 214219.

[16] White, F., Fluid Mechanics, 5th Edition, McGraw Hill, New York, NY, 2003.

[17] Ziolkowski, A., A method for calculating the output pressure waveform from an airgun, Geophysical Journal of the Royal Astronomical Society, 21, 1970, 137-161.

[18] Ziolkowski, A., An airgun model which includes heattransfer and bubble interactions, in SEG Annual Meeting, Dallas, Texas, 1982, 187-189. 